LA- - I0989-T

DE87 010835

\title{
An Experimental Measurement of Metal Multilayer X-Ray Reflectivity Degradation Due to Intense X-Ray Flux
}

\author{
Mary Y. P. Hockaday
}

\section{DISCLAIMER}

\begin{abstract}
This report was prepared as an account of work sponsored by an ageilcy of the United States Government. Neither the United States Government nor any agency thereof, nor any of their employees, makes any warranty, express or implied, or assumes any legal liability or responsibility for the accuracy, completeness, or usefulness of any information, apparatus, product, or process disclosed, or represents that its use would not infringe privately oxened rights. Reference herein to any specific commercial product, process, or service by trade name, trademark, manufacturer, or otherwise does not necessarily constitute or imply its endorsement, recommendation, or favoring by the United States Government or any agency thereof. The views and opinions of authors expressed herein do not necessarily state or reflect those of the United States Government or any agency thereof.
\end{abstract}




\section{ACKNOWLEDGEMENTS}

I would like to thank all the people who contributed to this project.

To my wise and guiding mentors, Dr. Robert Liefeld, Dr. Richard Blake, M. A. "Pete" Palmer, Dr. Robert Day, and Dr. Burton Henke, who all have helped me grow as a physicist.

To the PROTO II experimental team, "Pete" Palmer, Albert Widman, Dr. Rick Spielman, Dr. Bruce Hammel, who helped me figure out what I was looking at.

To Michael Selph, John Tobin, Joseph Chmielewski, Kurt Stetler, Stephan Baumgart, Frank Ameduri, and Robert Ripple, who shared their technical expertise.

To Karen Hindman, who worked very hard and did a great job on the figures. 


\begin{abstract}
AN EXPERIMENTAL MEASUREMENT OF METAL MULTILAYER X-RAY REFLECTIVITY DEGRADATION DUE TO INTENSE X-RAY FLUX BY

MARY YVONNE POTTENGER HOCKADAY, B.S., M.S.

Doctor of Philosophy in Physics

New Mexico State University

Las Cruces, New Mexico, 1986

Dr. Robert J. Liefeld, Chairman
\end{abstract}

The degradation of the $x$-ray reflection characteristics of metal multilayer Bragg diffractors due to intense $x$-ray $f l u x$ was investiqated. The $z$-pinch plasma produced by PROTO II of Sandia National Laboratories, Albuquerque, New Hexico, was used as the scurce. The plasma generated total $x-r a y$ yields of as much as $40 \mathrm{~kJ}$ with up to $15 \mathrm{~kJ}$ in the neon hydrogen- and helium-like resonance lines in nominal 20-ns pulses. Molybdenum-carbon, paladium-carbon, and tung̣sten- 
carbon metal multilayers were placed at 15 and $150 \mathrm{~cm}$ from the plasma center. The multilayers were at nominal angles of 50 and $10^{\circ}$ to diffract the neon resonance lines. The time-integrated $x$-ray reflection of the metal multilayers was monitored by $x-r a y$ f $11 \mathrm{~m}$. A fluorescerfiber optic-visible streak camera detector system was then used to monitor the time-resolved $x-r a y$ reflection characteristics of 135 \&- 2d tungsten-carbon multilayers. A large specular component in the reflectivity prevented determination of the rocking curve of the multilayer. Data for two separate shots were analyzed. For a pure neon gas shot at a power level of $420 \mathrm{TW}$, the reflectivity of the multilayer at $15 \mathrm{~cm}$ decayed rapidly with respect to the still-rising signal of the multilayer at $150 \mathrm{~cm}$. The onset $t$ ime of the decay corresponded to an integrated dose of $5.27 \mathrm{~J} / \mathrm{cm}^{2}$.

For a neon implosion onto a vanadium-doped polyacrylic acid foam target shot, detailed modeling was attempted. The spectral flux was determined with data from 5 XRD channels and deconvolved using the code SHAZAY. The observed decay in reflectivity was assumed to correspond to the melting of the first tungeten layer. A "conduction factor" of 82 was required to manipulate the heat loading of the first tungeten layer such that the time of melting corresponded to the observed 
decay. The power at destruction was $141 \mathrm{MW} / \mathrm{cm}^{2}$ and the integrated energy at destruction was $2.0 \mathrm{~J} / \mathrm{cm}^{2}$. 
TABLE OF CONTENTS

Page

List of Tables.......................... xi

List of Appendix Tables................... xii

List of Figures......................... $x i v$

List of Appendix Figures................... $x x$

INTRODUCTION. ........................... 1

PART I. ROOM TEMPERATURE CHARACTERIZATION OF METAL

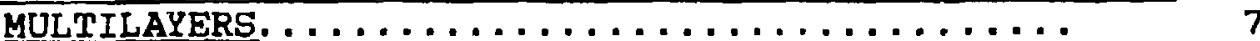

Chapter I-I PHYSICAL AND X-RAY CHARACTERIZATION... 8

Physical Characterization................ 8

x-Ray Characterization.................... 10

R vs. E curve....................... 11

R vs. $\theta$ curve................... 12

Chapter I-2 CALCULATIONS OF MULTILAYER REFLECTION PRORERTIES. ............................ 18

Darwin-Prins and Modified Darwin-Prins........ 18

Optical Thin-Film Theory................ 24

Data base.......................... 33

Carbon............................ 34

Tungsten. ....................... 35

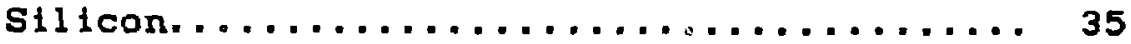

Molyabenum. ......................... 36

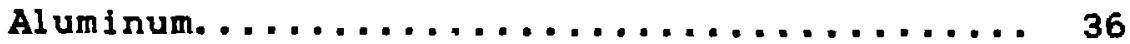

Boundary Layer Roughness.................. 36

SUMMARY FOR PART I....................... 41 
PART II. MULTILAYER CHARACTER IZATION UNDER INTENSE ELASH

X-RAY LOADING. . . . . . . . . . . . . . . . . . 42

Chapter II-1 THE SOURCE AND ITS CAPABILITIES...... 43

PROTO II............................ 46

Sandia Diagnostics...................... 52

Time-Integrated Diagnostics............. 52

X-ray pinhole cameras............... 52

Potassium acid-pthalate curved-crystal

spectrograph..................... 57

Grazing incidence spectrograph. .......... 57

Time-Resolved Diagnostics............... 59

8-ray diodes........................ 59

Thin-film bolometers.................61

Rough Calculation of Heating Capabilities of PROTO II............................. 65

Chapter II-2 TIME-INTEGRATED EXPERIMENTS........ 71

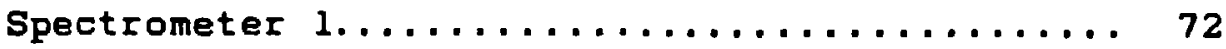

spectrometer 2...................... 72

Experimental Run..................... 80

Chapter II-3 TIME-RESOLVED EXPERIMENTS. ......... 89

The Experimental Design.................. 89

The Fiber-Bundle..................... 95

The Eluorescer....................... 104

The Fast Valve....................... 110

The Streak Camera.................... 111

The Timing........................ 114

The overall setup..................... 114 
Experimental Run..................... 117

Data........................... 124

Discussion....................... 131

Fluorescer conversion efficiency......... 131

spatial resolution................. 134

Spectral resolution................. 136

Chapter II-4 InCIDENT SPECTRUM. ............. 139

Post-Calibration of XRD Detectors............ 141

Bolometer and XRD Discrepancy............. 145

Anisotropy.......................... 150

Similarity of Time History............... 154

Conversion Efficiency................... 154

Chapter II-5 heATING MODEL. .............. 162

Energy Deposition..................... 162

Thermophysical Properties............... 172

Multilayer Reflection Properties.......... 173

Chapter II-6 Analysis. ................. 176

Shot $\# 1863 \ldots \ldots \ldots \ldots \ldots \ldots \ldots \ldots \ldots \ldots \ldots \ldots \ldots 176$

Shot $1872 \ldots \ldots \ldots \ldots \ldots \ldots \ldots \ldots \ldots \ldots \ldots \ldots \ldots 18 \ldots \ldots \ldots$

Discussion........................ 192

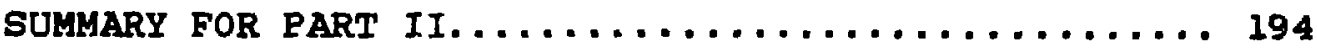

FUTURE WORK. . . . . . . . . . . . . . . . . 197

REFERENCES. . . . . . . . . . . . . . . . . . . . 199 
APPENDIX A. MULTILAYERS AND LABORATORY CU L $\alpha$

MEASUREMENTS. ...................... 208

APPENDIX B. MULTILAYER COMPUTER CODE. . . . . . . 215 
Table

Page

1. X-ray measurements on multilayer OVLA-130B-2 40

2. Summary of useful time-integrated neon gas 87 shots.

3. Summary of time-resolved data shots. 125

4. Densities of the step wedge with corre- 128 sponding values of exposure.

5. Time response measurements of NElll samples. 133

6. Comparison of four pure neon gas shots. 147

7. Comparison of three neon target shots. 149

8. Comparison of yields of two identical 155 bolometers at different angles to the source.

\section{LIST OF APPENDIX TABLES}

Table

Al Multilayers investigated. 210

A2 Measured multilayer reflection properties at 211 Cu-L $\alpha$ wavelength. 
Figure

Page

1. Schematic of metal multilayer $x-r a y$ diffractor, showing alterating isyers of ing and low $Z$ materiais deposited upon a substrate. $X$ rays are incident at angle $\underline{\theta}$ and are Bragg diffracted at angle $\underline{\theta}$.

2. A bi-layer periodic multilayer of alternating high and low Z materials of $N$ layerpairs or periods.

3. A typical reflectivity versus energy curve taken at Stanford Synchrotron Radiation Laboratory. LSM 82-152 was a 10 layer pair. tungsten-carbon multilayer with a tungsten thickness of $20 \&$ and a carbon thickness of 31 A. The curve was taken at an incident angle of 60 degrees.

4. Experimental configuration for measuring reflectivity as a function of angle.

5. Measured reflectivity versus angle curve at

Cu L wavelength for LSH 83-021, a tungeten-carbon multilayer of 2 d $\approx 70 \&$.

6. Schematic of a crystal containing an 20 infinite number of crystal planes. $T_{\text {f }}$ is the transmitted amplitude and $S_{j}$ is thereflected amplitude at an interfact.

7. A unit cell and volume structure factors, $m F_{1}$ and $m F_{2}$, for a bi-layer tungsten-carbon mutt il ayer:

8. Schematic of an m layer multilayer showing conventions for the layers and interfaces.

9. Schematic depicting electric and magnetic field vectors at the first two interfaces of a multilayer system.

10. Example of the roughness data obtained with optical profilometry for multilayer ovLA- 
130B-2. Information of the surface profile, the distribution of surface heights, and the autocovariance function assuming a Gaussian distribution of surface heights is obtained.

11. Time-integrated spectrum taken by M. A.

Palmer of Sandia Laboratories with a potassium ac ad pthalate curved-crystal spectrograph.

12. An artist's conception of the PROTO II Zpinch, pulse-power machine. Drawing is courtesy of Rick Spielman, Sandia National Laboratories.

13. Schematic of the gas nozzle/target assembly showing the configurations of the target. nozzle, return current posts, and electrical feed. Drawing courtesy of Sandia National Laboratories.

14. Spectral response of the two-layer $f$ ilm pack for the $x-r a y$ pinhole cameras fielded by $M$. A. Palmer.

15. Example of a kiloelectronvolt pinhole image of the PROTO II source.

16. Absolute spectral sensitivity of the carbon mirror pinhole camera. Transmission of the 2000-8 gold filter has been taken into account.

17. Spectral sensjtivity of XRD Channels 1-3. assuming $1-\mathrm{cm}^{2}$ source and taking into account all geometrical factors.

18. Spectral sensitivity of XRD Channels 4, 5 , and 7, assuming a $1-\mathrm{cm}^{2}$ source and taking intio account all geometrical factors.

19. Spectral energy response of the two thinfilm bolometers fielded by D. L. Hanson.

20. Estimated spectral shape for the $x$ rays produced by PROTO II. 
21. General schematic of the two spectrometers 73 used at PROTO II.

22. Photograph of the components of Spectrometer 1 used in the time-integrated experiments.

ij. Photograph of the components of spectrometer 2 used in the time-integrated experiments.

24. Revised schematic of the two multilayer spectrometers used at PROTO II.

25. Transmission of "best" filter combination of $8 \mu \mathrm{m}$ of $\mathrm{Kimfol}$ and $0.5 \mathrm{mil}$ of $\mathrm{Al}$ as the postfilter and $2 \mu_{m}$ of $K i m f o l$ and $0.3 \mathrm{mil}$ of Al for the prefilter.

26. Spectral sensitivity of final film configuration used in the multilayer spectrometers.

27. Diagram defining parameters necessary to calculate the flux through the fluorescerfiber optic-streak camera system.

28. Derivation of $\underline{\underline{q}} \Psi$ and $\underline{\mathrm{d} \theta}$.

29. Spectral attenuation as a function of wavelength of the DBF :iber for three different wavelength ranges.

30. Configuration of the fiber bundle ends.

31. Photograph of the iber bunde holder-stage 100 assembly for Spectrometer 1 .

32. Photograph of the $f$ iber bundle holder-stage asssembly for Spectrometer 2 .

102

33. Spectrophotometer scan of BARR interference filter showing percent transmission as a function of wavelength.

34. The effects of the fluorescer and inter.

ference filter on the spatiai resolution. ax, of the system. The spot size on the photocathode is less than the outer diameter of the fiber: therefore, the spatial resolution is determined at the fluorescer end of the system. 
Figure

Page

35. The EG\&G streak camera. Shown here are the components of the film back, the microchannel plate (MCP), the streak tube housing, and the $f$ iber optic bundle mount.

36. Schematic of the streak camera timing sequence.

37. $x$-ray, signal, and trigger paths of the two 116 multilajer spectrometers.

38. Photograph of the two $t$ ime-resolved multilayer spectrometers as configured during the run.

39. Photograph of the PROTO II vacuum chamber. multilayer spectrometers and the associated radial diagnostics.

40. Print of streak record of shot \#1863 showing data, combs, impulse, step wedge, and the space that separates the two sections of fiber of Spectrometer 2 .

41. Compressed streak from Spectrometers 1 and 2 for Shot \#1863. Data were smoothed in exposure space using AHARE adaptive filtering eode.

42. Determination of spatial resolution of the 135 fluorescer-fiber bundle-interference filter system with measured thicknesses. The spot size on the streak camera's photocathode is greater than the outer diameter of the optical fiber.

43. Theoretical R vs. E curve for a tungstencarbon multilayer of $2 \mathrm{~d}=135 \mathrm{~A}$ at an incidence angle of 6.25 degrees.

44. Deconvolved spectrum from XRD data for Shot \# 1872 .

45. Heasured and assumed quantum efficiencies for gold photocathodes of Channels 4, 5, and 7. Straight 1 ines indicate presence of nongold absorption edges. 
Figure

Page

46. Comparison of original and revised XRD 146 response curves for Channels 4,5 , and 7 .

47. X-ray pinhole photograph of shot \#1872 151 showing the target and the "spilier web" target holder. The target holder blocks the $x$ rays enitted from the plasma from reaching the diagnostics at angles closest to the zaxis.

48. Angular placement of $x$-ray diagnostics on PROTO II.

49. Comparison of line and total spectral power determined by XRD channels and bolometers for Shot \#1872. XRD line signal scaled by 9.9 and the XRD total yield signal scaled by 8.0 .

50. Implodirg gas shell of inner radius $I_{1}$, outer radius $r_{2}$, and length, $\underline{l}$, and cytindrical target of radius, $I_{f}$.

51. Shot \#1872 input current and calculated radius and velocity for a shell with $\bar{E}=6.5$ $x 10^{\text {I6 }}$ particies per cubic centimeter.

52. Method for calculating absorptance, $\mathbf{A}_{j}$, in layer $i$ by assuming that the multilayer is an absorber subjected to an incident beam of intensity $i_{0}$. The transmittance of layer $i$ is given by

158

161

164

53. Reflectivity versus photon energy curve for a 21-layer, tungsten-carbon multilayer.

54. Absorptance calculated by the absorber and 168 Poynting amplitude methods for the specular reflection region.

55. Absorptance calculated by the absorber and the Poynting amplitude method for the Bragg reflection region.

56. Absorptance calculated by the absorber and the Poynting amplitude method for the region far from the Bragg and specular reflection regions. 
57. Simplifled flow chart of the combined multi175 layer and heating computer code.

58. Spectra taken with the KAP 5 pectrograph fielded by $M$. $A$. Palmer for three neon gas shots showlrig the non-repeatability.

59. Total bolometer and multilayer spectrometer cata for Shot *1863. The lefi-hand verticai axis is the total Power oetermined by the bolometer signal in $4 \pi$ sr. The right-hand vertical axis is the exposure on the streak camera film.

E0. Total bolometer and multilayer spectrometer data. Spectrometer 2 signal has been scaled to correspond to the other two signals.

61. The integrated total bolometer signel and the data from the two multilayer spectrometers for Shot \#1863. The left vertical axis gives the total integrated energy. The right axis gives the exposure on the streak camera film for the two compressed streaks.

62. Total bolometer and multilayer spectrometer data for shot 1872. The left vertical axis is the total power in $4 \pi$ sr determined by the bolometer signal. The right vertical axis is the exposure on the streak camera film for the multilayer spectrometers.

63. The integrated total bolometer signal and the data from the two multilayer spectrometers for Shot $\# 1872$. The left virtical axis gives the total integrated energy, while the right vertical axis gives the exposure on the streak camera film for the two compressed streaks.

54. Reflectivity curve around the Bragg diffractIon peak for the initial multilayer parameters and for the expanded multilayer parameters at the melting temperature of the first tungsten layer for Shot 1872 . 
65. Multilayer Spectrometer 2 streak camera data 191 and the calculated temperature profile as a function of time for the two top tungsten layers. The right axis is exposure on the streak camera film for the two spectrometers. The left axis is the temperature of the layers.

\section{LIST OF APRENDIX FIGURES}

Eigure

Page

Al. Sectioning and labeling used for three-inch 209 multilayer disks. 
INTRODUCTION

Metal multilayer x-ray diffactors, also known as layered synthet ic microstructures (LSM's) and evaporatedsputtered multilayers (ESM's), are alternating layers (5200 \& thick) of high and low atomic number materials deposited upon a substrate, as depicted in Figure 1. The resulting periodicity in the electron density gives rise to Bragg diffraction analogous to that in natural crystais.

The earliest efforts to create these artificial Bragg diffractors were those of Koeppe (Deubner, 1930) and Deubner (1930), who were unable to observe diffraction lines from the multilayer superlattice. Dumond and Youtz (1935: 1940) did observe diffraction from such a superlattice of copper and gold, but the layers interdiffused in a day or two at room temperature. Dinklage ( 1967 ) was successful in creating iron-magnesium multilayers with a shelf life of about a year.

In the 1970 multilayer construction was improved by, among others, Eberhard Splller at IBM (Spiller et al. 1974 and 1976; Haelbich et al.. 1978), who used evaporation techniques, and Troy Barbee at Stanford University (Barbee and Keith, 1978; Underwood et a1., 1979), who used magnetron sputtering. Since then the field has grown rapidly in both applications and 


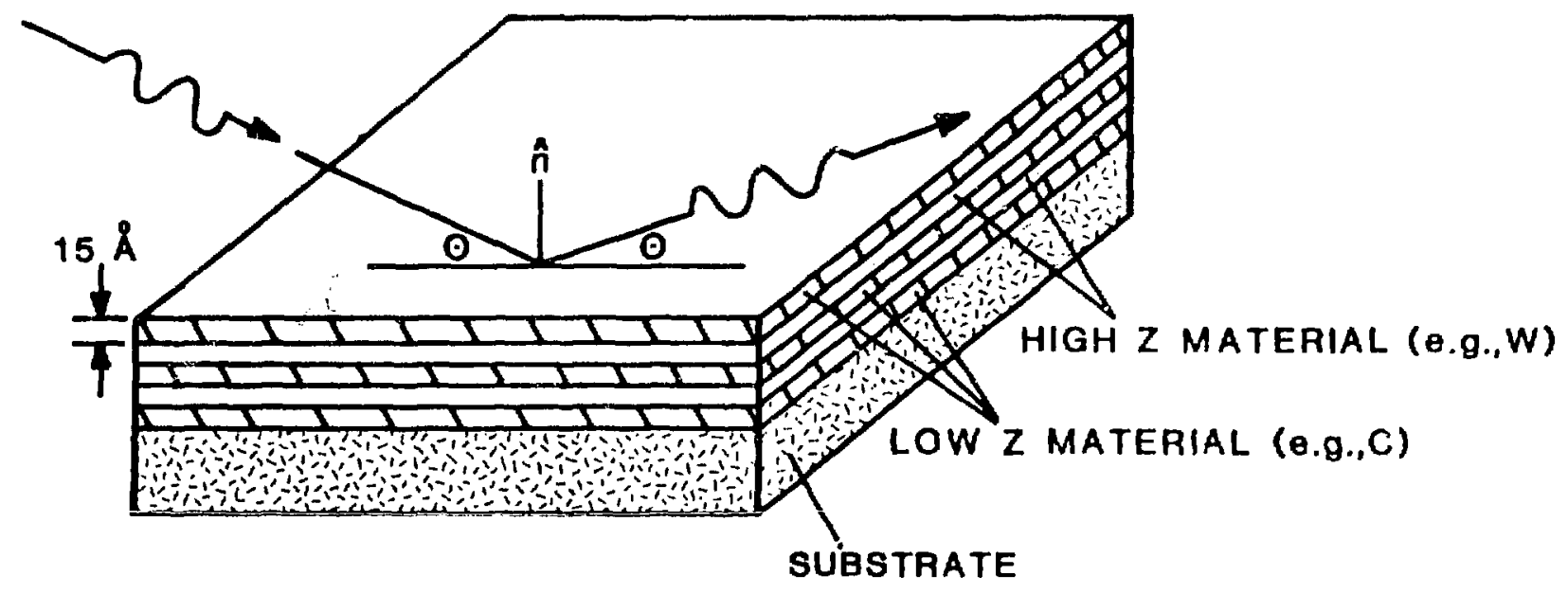

Figure 1. Schematic of metal multilayer $x-r a y$ diffractor, showing alternating layers of high and low $Z$ materials depositid upon a substrate. X rays are incident at angle $\underline{\theta}$ and are Eragg-diffracted at angle $\underline{\theta}$. 
availability of multilayers.

There are three main reasons for curcent interest in metal multilayers. First, the ability to vary the 2dspacing gives the experimenter the capability to design a multilayer that can be optimized for a prescribed energy range. Second, since multilayers can be constructed with 2d-sparings between 25 and 2008 , they complement the Langmuir-Blodgett molecular multilayers (70-130 \&) and natural crystals (less than $\sim 35$ \&) and extend the range for spectroscopic applications. The third reason is that the production process of evaporation or sputtering allows the deposition of multilayer coatings on curved substrates (Henry et al., 1982: Nagel et al.. 1981). which permits construction of a variety of $x-r a y$ optical devices that are useful in plasme $x$-ray spectroscopy. In addition, multilayers do not have to be bi-layer periodic structures but can be graded in depth or laterally to meet bandpass needs (Lee, 1983; Nagel et al., 1981 and 1982). Even solid Fabry-Perot etalon systems can be fabricated (Barbee and Underwood, 1983; Bartlett et al.. 1985). A recent conference on multilayer $x$-ray optics (Proc. SPIE Vol. 563, 1985) reviews the status of the field today.

For high-temperature plasma diagnostics, it is neceseary to measure the $x$-ray characteristic line 
radiations of highly stripped ions produced in the plasmas. Since metal multilayers are now available with the appropriate rargo of 2 da-spacings, they are prime candidates for certain kinds of high temperature plasma d.agnostics (Gilfich et al., 1982: Day and Barbee, 1985). For very intense plasmas, the possibility arises that the radiation flux incident on the multilayer may change the multilayer $x-r a y$ reflection properties. The determination of $f$ lux levels at which the multilayer reflection properties change would permit compensation for the multilayer response, allowing for a more precise characterization of the plasma. In addition, normal incidence multilayer mirrors are being investigated for use in $x$-ray laser cavities (Ceglio et al.. 1985) and their performance under fast intense flux conditions needs to be investigated.

A preliminary calculation for a z-pinch plasma radiating $30 \mathrm{~kJ}$ of $x$ rays indicates that the sudden temperature rise in a multilayer of interest at a useful distance would be on the order of a few electronvolts $\left(0.86 \mathrm{eV}=10,000{ }^{\circ} \mathrm{K}\right)$. Material properties in this temperature range produced by thermal pulses are not well understood. For this reason, it is necessary to perform experiments and model the results to get a better understanding of these material properties. This problem of 
characterizing the changes in multilayer $x-r a y$ diffraction properties, both experimentally and through modeling, as the multilayer is subjected to intense bursts of $x-r a y$ radiation, is the subject of this alssertation.

The work is in two parts. Part I describes the room temperature characterization of metal multilayers, laying the foundation for understanding multilayers. It consists of two chapters and a summary. Chapter I-I presents the physical and experimental x-ray characterization for the metal nultilayer Bragg diffractors. Chapter I-2 discueses the different methods used to model such diffractors' room temperature $x-r a y$ reflection characteristics and describes the method chosen for analysis. It also presents the comparison of some experimental results with the chosen model. Part II describes the characterization of multilayers under intense flash-heat loading by $x$ rays. It has $s i x$ chapters. Chapter II-1 discusses the $x$-ray source criteria necessary to carry out the planned experiments as well as the specific characteristics of the source chosen. Chapter II-2 presents the time-integrated flash $x$-ray experiments and their results. Chapter II-3 discusses the time-resolved $x$-ray experiment, while Chapter II-4 discusses how the spectral intensity of the 
x-ray radiation was determined. Chapter II-5 discusses the heating model used. In Chapter II-6 analys is of the time-resolved experimental data is discussed. A summary of Part II is then presented. Finally, a discussion of future work is given. 
PART I

ROOM TEMPERATURE CHARACTERIZATION OF METAL MULTILAYERS 
CHAPTER I-1

PHYSICAL AND X-RAY CHARACTERIZATION

\section{Physical Characterization}

Metal multilayers are alternating layers of materials of high and low atomic number, $z$, deposited onto substrate. Of particular interest is the simplest multilayer, which has a repeating structure of two layers, as depicted in Figure 2. Each high $z$ layer has the same thickness, ${ }^{d} H^{\prime}$ as every other high $\underline{z}$ layer, and every low $\underline{Z}$ layer has the same thickness, $d_{L}$. The combination of one high $\underline{z}$ layer and one low $\underline{z}$ layer is defined as a layer pair, which in this case is also one period of the multilayer. Each period has a thickness.

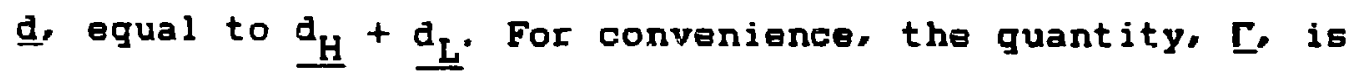
defined as the ratio of the high z-layer thickness to the period thickness.

The materials of a metal multilayer are alternatively deposited in a vacuum system onto a substrate, usually a piece of polished silicon crystal. The layer composition is determined by the manufacturing process. Ideally, a multilayer would have perfectly smooth interfaces and be deposited on a perfectly smooth and flat substrate. However, during production the depositing material may combine with other materials in 

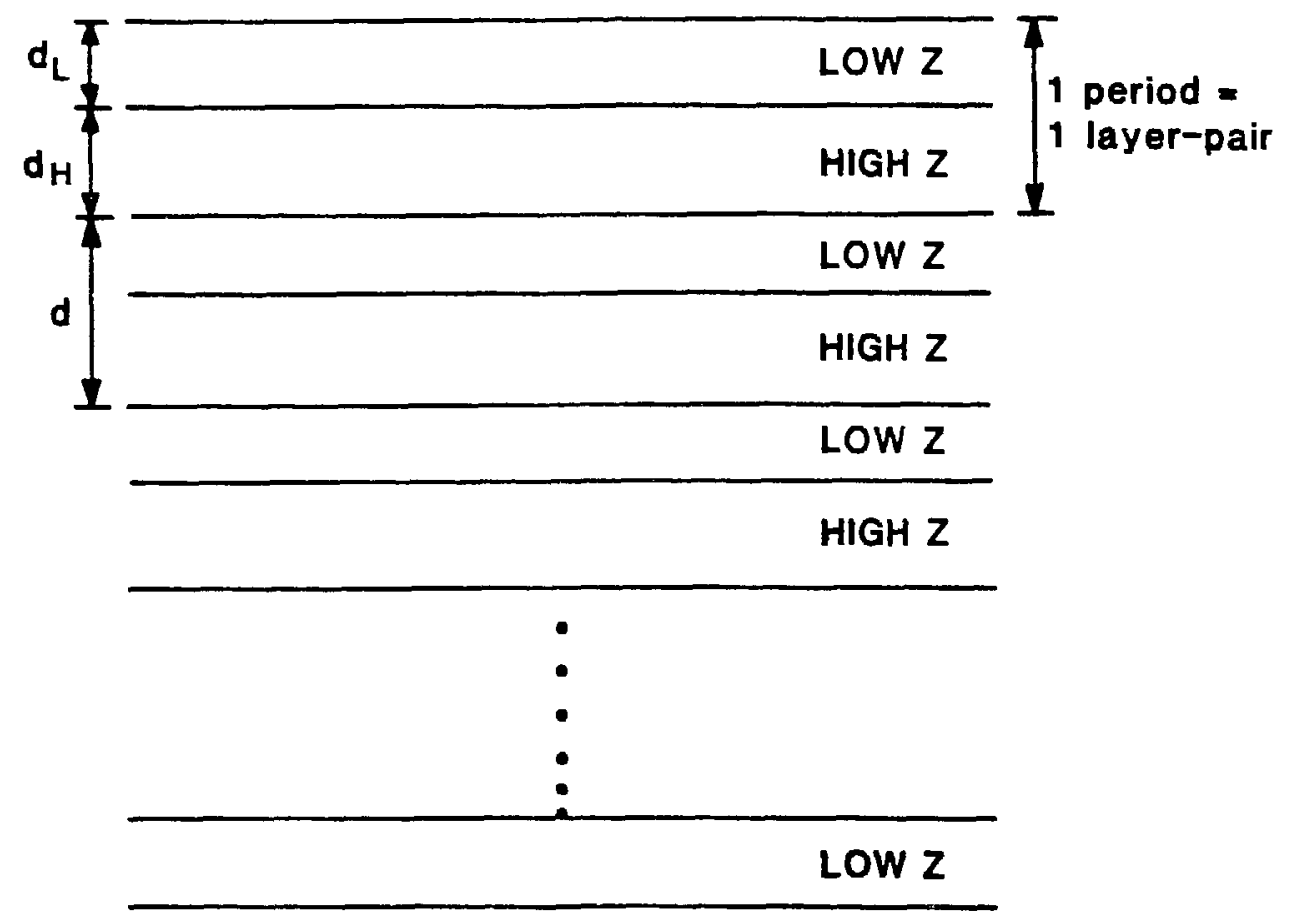

HIGH Z

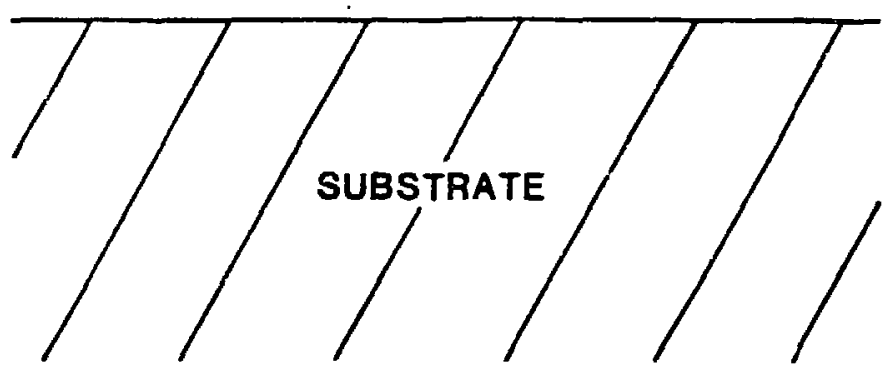

Figure 2. A bilayer perlodic multilayer of alternating high and low Z materials of $N$ layer-pairs or periods. 
the vacuum system to form impurs or transition layers. This type of structure can be detected through Auger depth profile analysis (Rachocki et al.. 1984), x-ray diffaction analysis (Takagi et al., 1985), careful fitting of Bragg $x$-ray data taken at several wavelengthe (Henke et al., 1985 and 1986), transmiseion electron microscopy, other techniques. Manufactured multilayers have layers and substrates that are not perfectly smooth and have roughness on the order of one or two atomic heights (Underwood and Barbee, 1981a and b). Such roughness degrades the $x-r a y$ reflection properties of the multilayers.

The physical thickness of the individual layers in the multilayer can be determined by in situ monitoring (Spiller et al., 1980) during the manufacturing process. Careful messurement of the x-ray reflection properties of the completed multilayer determines only average values for the physical thicknesses of the composite layers.

\section{X-Rey Characterization}

When a beam of $x$ rays is incident upon a multilayer near the Bragg angle, it is diffracted. The intensity of the diffracted beam varies according to the angle of incidence and its energy or wavelength. The $x-r a y$ reflectivity of a multilayer is described by the curve of 
the ratio of the diffracted intensity over the incident intensity as a function of angle when the incident energy is fixed, or as a function of energy when the incident angle is fixed.

For either description, the positions of the diffraction peaks are given by Bragg's law, $n \lambda=2 d \sin \theta$.

where $\underline{n}$ is the order of the diffraction, 2 is the incident wavelength, d is the a-spacing equal to the effec$t$ ive thickness of a period of the multilayer, and $\theta$ is the incident angle. In addition to the Bragg diffaction peaks there may be a specularly reflected beam. Measurement of the reflectivity curves can be done for both deseriptions.

R vs. E Curve

To measure the $x$-ray reflection curve of a multilayer as a function of energy at a fixed angle, a continuously tunable wavelength source such as a synchrotron is necessary. The beam mechanics of a synchrotron light source are designed so that the $x-r a y$ beam is incident onto the multilayer at one fixed angle. The energy of the beam is stepped by use of a monochromator. and the diffracted intensity is measured at each energy setting point. The diffracted intensity is normalized by 
dividing by the incident intensity.

A typical experimental R vs. E curve taken at the Stanford Synchrotron Radiation Laboratory is shown in Figure 3 (Bartlett et al.. 1985). This particular curve is for a 10-layer pair, tungsten-carbon multilayer with a tungsten thickness of $20 \AA$ and a carbon thickness of $31 \AA$ at an incident angle of 60 degrees. The reflection properties of a multilayer are usually reported as the energy at which the peak was observed, the full width at half maximum (FWHM) of the reflection curve, the area under the reflection curve or $R_{E^{\prime}}$ and the peak reflectivity $\underline{\text { P. }}$

R vs. $\theta$ Curve

To measure the $x-r a y$ reflection curve as a function of angle, a monochromatic, perfectly parallel $x-$ ray beam is needed. A synchrotron source with a good monochromator produces an $x-r a y$ beam that is nearly monochromatic with very little beam divergence. Large beam divergence contributes to the apparent width of the reflection curve. For routine $x$-ray characterization of multilayers, use of synchrotron facilities is difficult to obtain and very expensive. In the kilo-electronvolt energy region, the width of a multilayer's diffraction pattern is very large ( $\sim 5 \mathrm{mrad}$ ) compared with a natural 


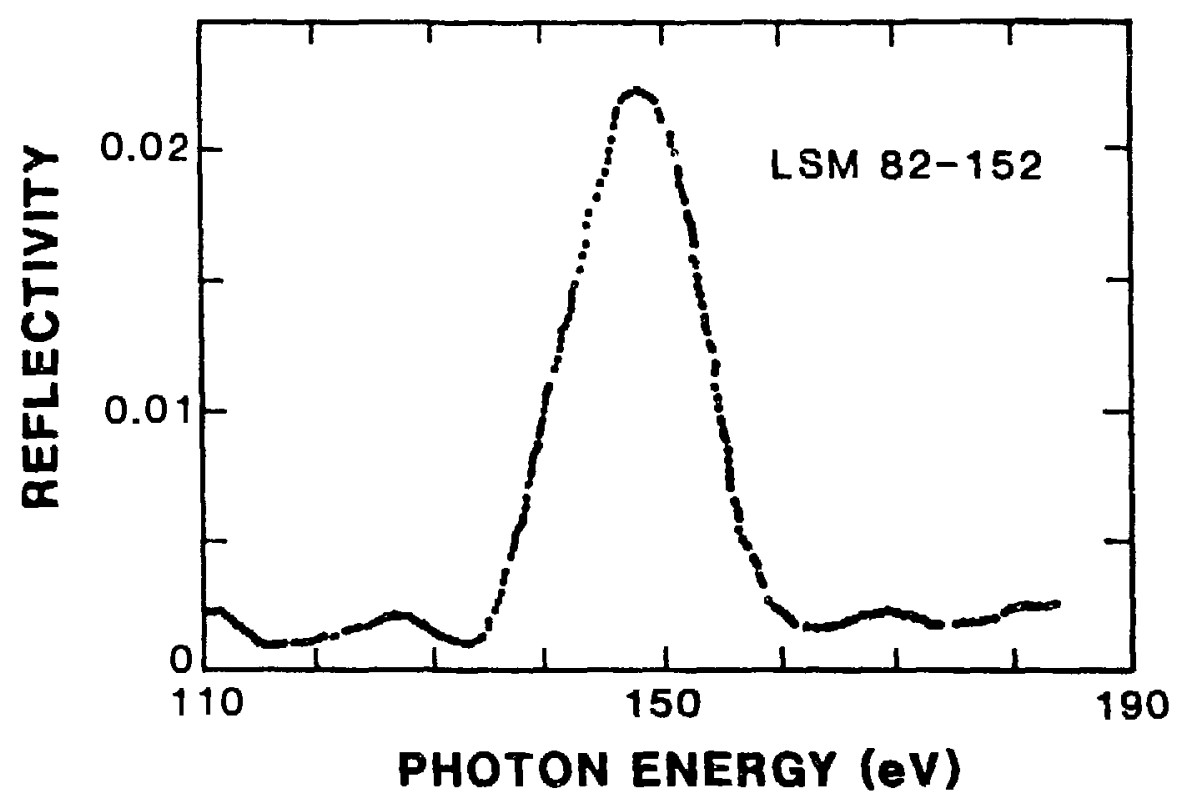

Figure 3. A typlcal reflectivity versus energy curve taken at Stanford Synchrotron Radiation Laboratory. LSH 82-152 was a 10-layer pair, tungsten-carbon multilayer wth a tungsten thickness of 208 and a carbon thickness of 31 . The curve was taken at an incident angle of 60 degrees. 
crystal, e.g., a potassium acid pthalate (KAP) crystal ( 0.3 mrad). If the beam is first incident upon such a crystal at the proper angle, then the crystal not only helps to monochromatize the $x$-ray beam, but it limits its divergence, thereby contributing little to the measured width of the multilayer's diffraction pattern. This technique was used to measure the $x-r a y$ reflection curve as a function of angle. The experimental setup is schematically shown in Figure 4. A KAP crystal was held fixed and used to monochromatize arid limit the beam divergence. The purified beam was then incident upon the multilayer, which was rotated through a succession of angles. The beam diffracted off the multilayer was measured by a gas flow proportional counter located at twice the angle of the multilayer.

A typical $\underline{R}$ vs. $\theta$ curve measured for a tungstencarbon multilayer with a d-Epacing of $70 \mathrm{~g}$ and 120-layer pairs for an incident energy of $930 \mathrm{eV}$ ( $\mathrm{Cu} L_{\alpha}$ ) is shown In Figure 5. The $x$-ray reflection properties are chacacterized by the peak angle, $\theta$ and the peak reflectivity, $\underline{P}$, the FWHM of the curve. The integral under the curve in radians is known as the integrated reflectivity, $\quad \mathrm{R}_{\theta^{\prime}}$ This integrated reflectivity is related to the corresponding integrated reflectivity of the energy description, $R_{E^{\prime}}$ by 


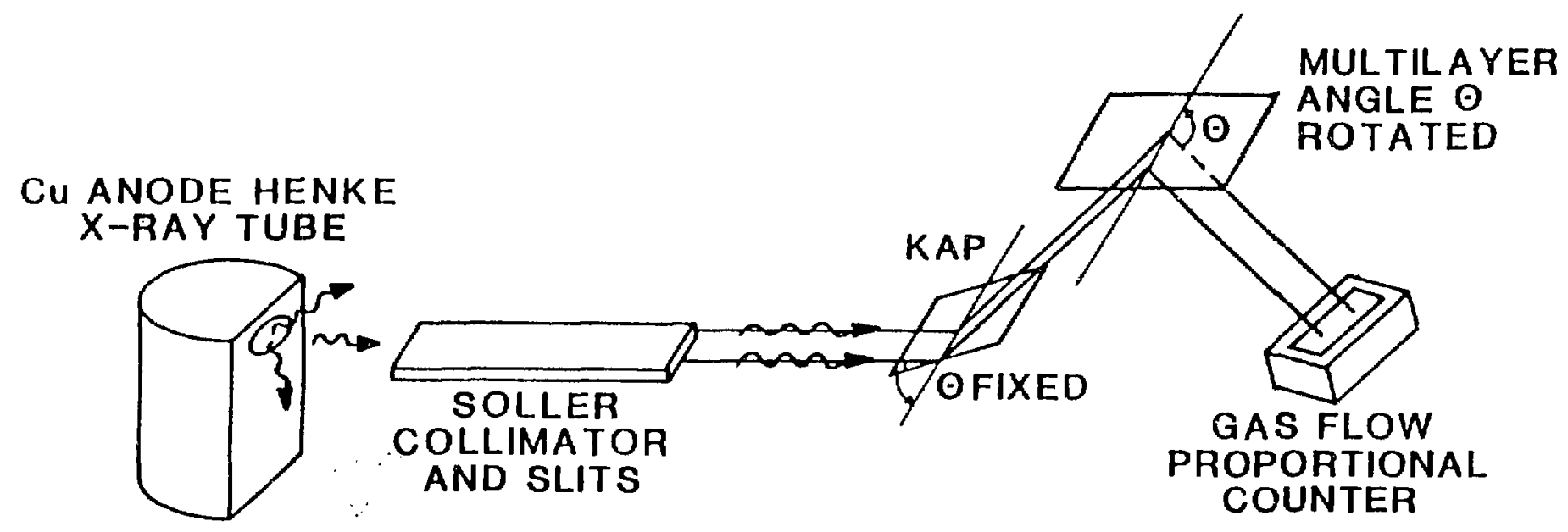

Figure 4. Experimental configuration for measuring reflectivity as a function of angle. 


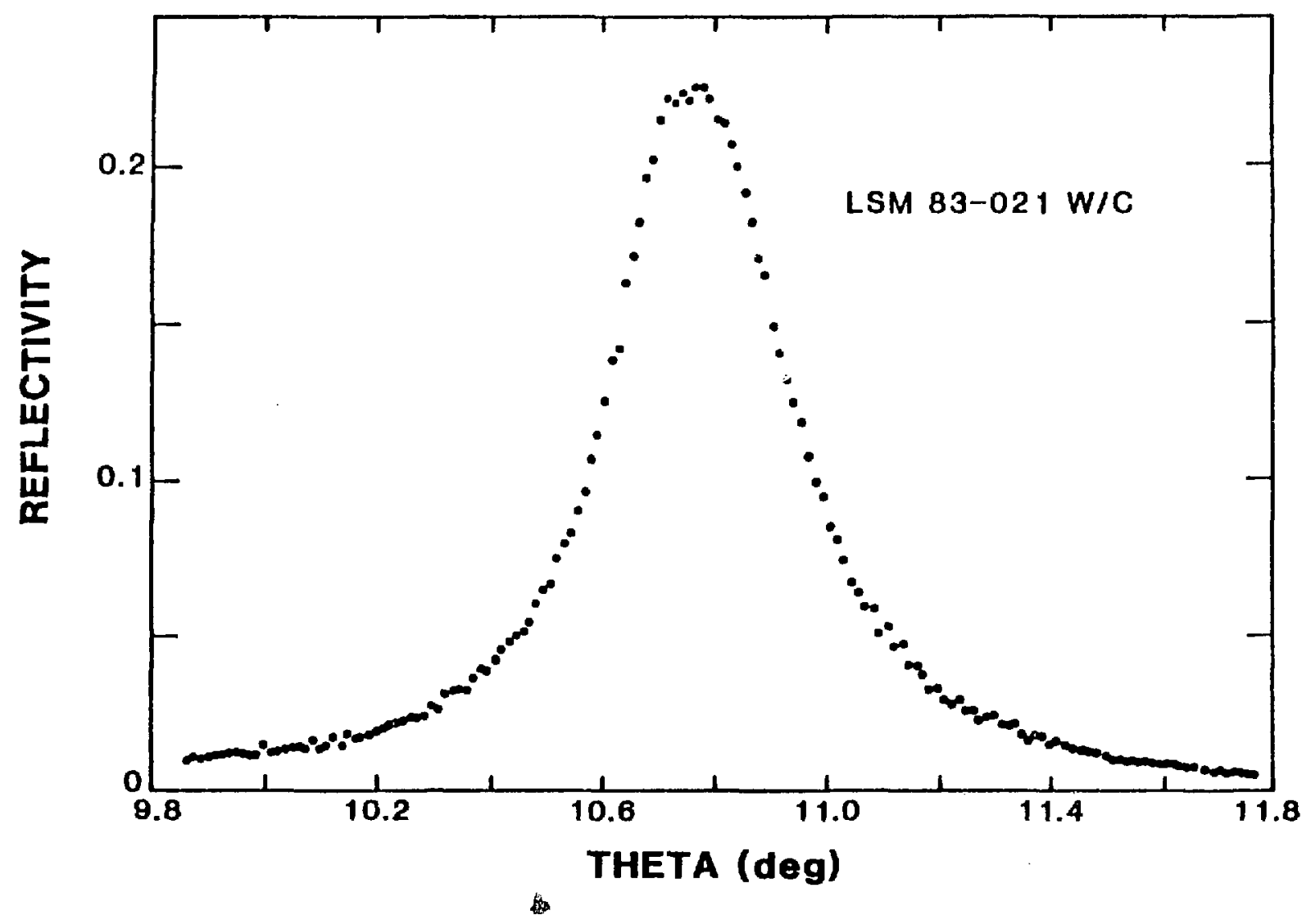

Figure 5. Measured reflectivity versus angle curve at Cu L wavelength fgr LSM 83-021, a tungeten-carbon mult $f$ layer of $2 \mathrm{~d} \approx 70$. 
$R_{E}=R_{\theta} \frac{d E}{d \theta}=R_{\dot{\theta}}\left(\frac{n h_{c}}{2 d} \operatorname{Cot}(\theta) \operatorname{Csc}(\dot{\theta})\right)$.

where $\underline{n}$ is the order, $\underline{h}$ is Planck's constant, $\underline{c}$ is the speed of light, and $\dot{\theta}$ is the Bragg angle.

Al multilayers used in this investigation were measured at $\mathrm{Cu} \mathrm{L}_{\alpha}$ radiation with this technique. A summary of these measurements on the multilayers used in this investigation is given in Appendix $A$. The $\mathrm{Cu} \mathrm{L}_{\alpha}$ radiation was chosen because it was the most convenient radiation near the peak of the radiation that was to be observed during the impulse heating phase of this investigation.

In application, a source may not be monochromatic or give off a perfectly paraliel beam of $x$-ray radiation. In this case, the multilayer diffraction is more complicated, and care must be taken in the the interpretation of the intensity received at the detector. 
CHAPTER I-2

CALCULATIONS OF MULTILAYER REILECTION PROPERTIES

Calculations that determine the $x$-ray reflection and transmission of multilayer optics can be categorized into two approaches. The first is to extend the dynamical theory of diffraction of $x$ rays from crystalline materials (Compton and Allison, 1935: Zachariasen, 1945) to metal multilayers (Unilerwood and Barbee, 1981a and b; Lee, 1981; Henke et al., 1985). The second approach is to solve the electromagnetic boundary-value problem for the steady-state electric andor magnetic field amplitudes at successive interfaces or layers of the multilayer. This approach has been used extensively in optical thin-f1lm theory (Born and Wolf, 1983; Weinstein. 1947) and can be used in the $x-r a y$ region with the incluEion of absorption (Parratt, 1954; Berning, 1963). These two approaches have been shown to be equivalent (Lee, 1981; Perkins and Knight, 1984) for non-crystalline mult 11 ayers.

\section{Darwin-Prins and Modified Darwin-Prins}

The Darwin-Prins method given here follows that presented by Compton and Allison (1935) and is based on the calculations of $x-r a y$ reflectivity for natural 
crystals.

The basic formulism begins with $x$ rays incident on an infinite set of planes or layers of a crystal, as depicted in Figure 6 . The incident wave amplitude is denoted by $\underline{T}_{0}$ and the reflected amplitude by $s_{0}$. The reflected and transmitted amplitudes, $\underline{S_{\underline{r}}}$ and ${\underline{T^{\prime}}}$ at a

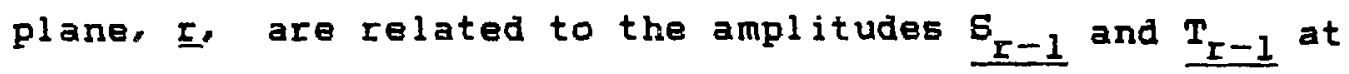
the preceding plane, $\underline{r-1}$, by the equations

$T_{I}=(1-i \sigma) T_{I-1} \exp \left(-\frac{-i 2 \operatorname{dasin} \theta}{\lambda}\right)-i s S_{I} \exp \left(-\frac{4 \pi \operatorname{tag} i n \theta}{\lambda}\right)$

and

$S_{I}=-i s T_{I}+(1-i \sigma) S_{I+1} \exp \left(-\frac{2 \pi d s i n \theta}{\lambda}\right)$

where $\underline{E}$ is the fractional amplitude that is reflected at a plane, $\underline{Q}$ is the fractional amplitude that is absorbed in the layer, $\underline{a}$ is the layer's thickness, $\theta$ is the Incldent angle, and $\underline{\lambda}$ is the incldent wavelength of the radiation. The parameters 5 and $Q$ are related to the atomic scattering factors ${\underline{f_{1}}}_{1}$ and $\underline{f_{2}}$ and the crystal structure factors $F_{1}$ and $F_{2}$ (Henke, 1981 a and b) by the equat ions

$\sigma=\frac{-r_{0} \lambda}{\sin \theta} n\left(f_{1}+i f_{2}\right)$ and $s=\frac{-r_{0} \lambda}{\sin \theta} \cdot\left(F_{1}+i F_{2}\right) P(2 \theta)$,

where $I_{0}$ is the classical electron radius, $\frac{n\left(f_{1}+i f_{2}\right)}{\text { is }}$ the atomic scattering factor per unit volume, $\left(F_{1}+i F_{2}\right)$ is the structure factor per unit volume, and $\underline{\underline{p(2 \theta)}}$ is the polarization factor. The polarization factor is equal to 


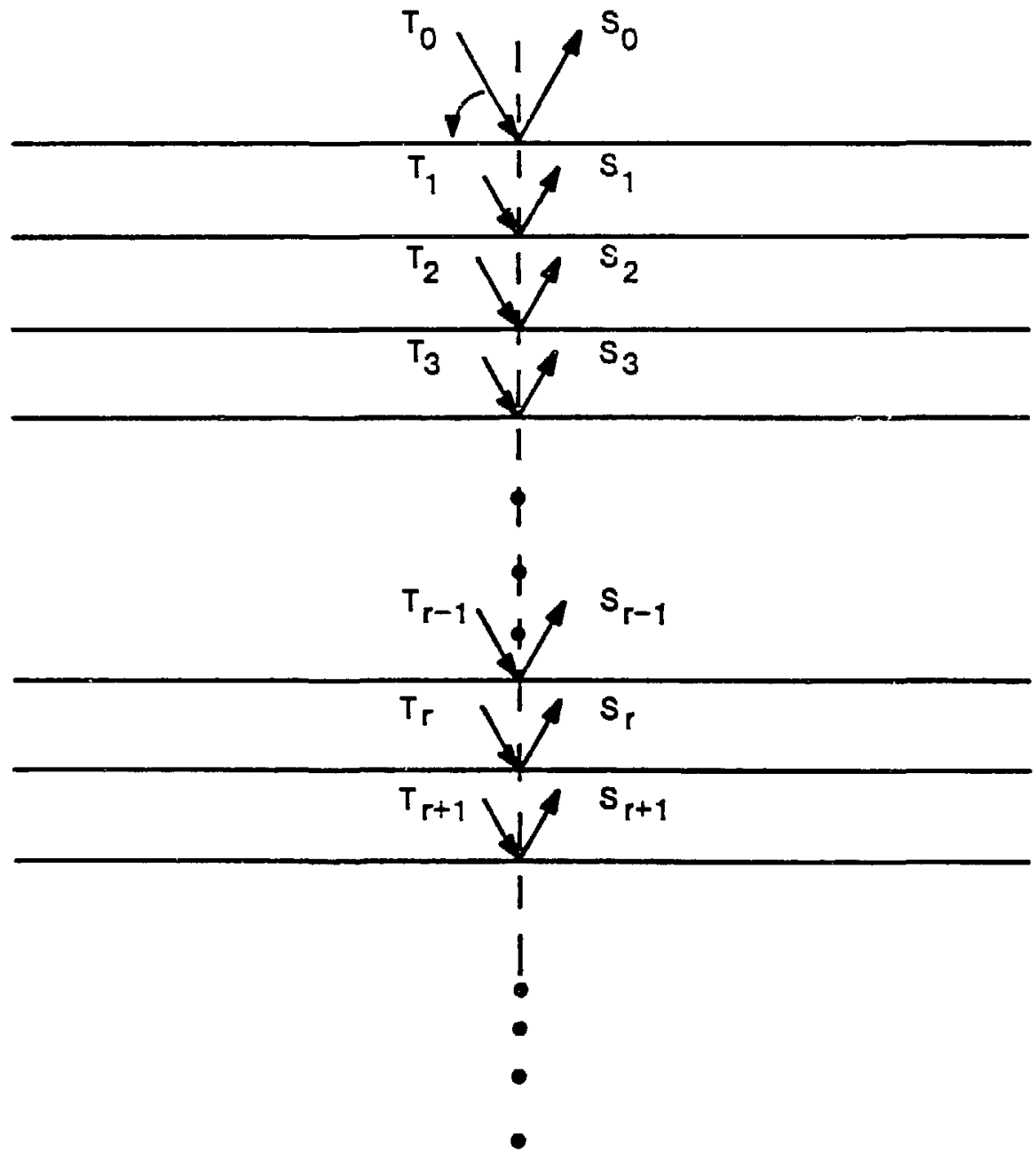

Figure 6. Schematic of a crystal containing an infinite number of crystal planes. If is the transmitted amplitude and $S_{j}$ is the reflected ampitude at an interface. 
unity for $E$ polarization and equal to $\cos 2 \theta$ for $D$ polarization.

The ratio of the reflected amplitude to the incident amplitude is derived by solving Egs. (2a) and (2b) for the case in which $\theta$ is near the Bragg angle, $\theta_{B}$. This solution is given by

$\frac{S_{0}}{T_{0}}=\frac{s}{(\sigma+\xi) \pm \sqrt{(\sigma+\xi)^{2}-s^{2}}}$,

where $\mathbf{A}$ is defined by the equation

$\xi=\frac{2 \pi d}{\lambda}\left(\sin \theta-5\right.$ in $\left.\theta_{B}\right)$, and

where $\theta_{B}$ is the Bragg angle. The reflectivity is calculated from the equation

$R=\left|\frac{S_{0}}{T_{0}}\right|^{2}$

for an infinite crystal or multilayer.

Henke et al. ( 1985 ) extend this result to the case of finite crystals or multilayers. The resulting equation for the Modified Darwin-Prins is

$\frac{s_{0}}{T_{0}}=\frac{\frac{s_{0}}{T_{0}}\left(1-x^{2 N}\right)}{\left(1-\left(\frac{s_{0}}{T_{0}}\right) x^{2 N}\right)}$.

where $\underline{N}$ is the number of planes and $\underline{x}$ is given by $x=(-1)^{n} \exp \left(-\left( \pm \sqrt{\sigma^{2}-(\sigma+\xi)^{2}}\right)\right.$,

and $\underline{n}$ is the order of the diffraction. The \pm sign of the 
radical is chosen such that the real part of $\underline{x}$ is less than one to consarve energy.

For amorphous multilayers (Henke et al., 1985 and 1986), the unit cell is one period or repeating structure. For example, for a tungsten-carbon multilayer the unit cell can be depicted as one layer of tungsten and one layer of carbon, as shown in Fig̣ure 7 . The structure factor sum or integral over the atoms in the unit cell is referenced to the displacement from some plane of symmetry. If the layers are crystalline is nature, this unit cell must be modified. The crystallinity will add extra diffraction effects in addition to those that are given by the superstructure of the multilayer.

This method is an efficient way of looking at purely periodic multilayers. In addition, density gradients representing roughness or transition layers can easily be incorporated into the unit cell as long as each period exhibits the same behavior throughout the multilayer. This method does not include the effect of a substrate, which is very small when the number of periods is large. In addition, the absorption in any particular layer is not apparent. 


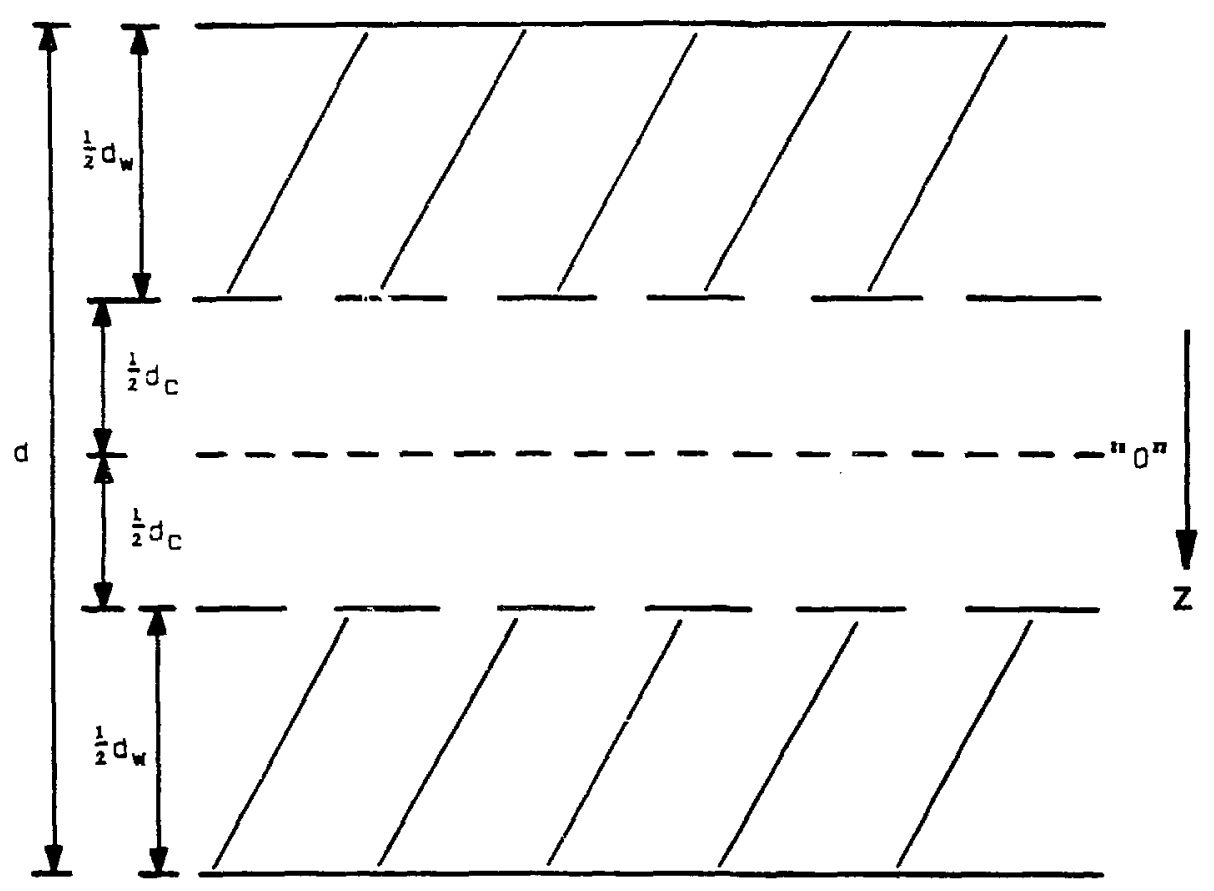

$$
\begin{aligned}
m F_{1} & =\int_{-d / 2}^{+d / 2}\left(n_{c}(z) f_{1_{c}}+n_{w}(z) f_{1_{w}}\right) \cos \left(\frac{4 \pi z}{\lambda} \sin \theta\right) d z \\
& +d / 2 \\
m F_{2} & =\int_{-d / 2}\left(n_{c}(z) f_{2}+n_{w}(z) f_{2}\right) \cos \left(\frac{\Delta \pi z}{\lambda} \sin \theta\right) d z
\end{aligned}
$$

Figure 7. A unit cell and volume structure factors, $\mathrm{mF}_{1}$ and $\mathrm{mF}_{2}$, for a bilayer tungsten-carbon multilayer. 


\section{Optical Thin-Film Theory}

Optical thin-film theory has been used for many years to calculate the optical properties of dielectric multilayers used as mirrors and interference filters in the visible portion of the spectrum. Figure 8 depicts a multilayer system and the labeling conventions that will be followed in this work. The multilayer consists of $m$ layers, including the substrate. Medium $\underline{m+1}$ is the exit mediuni. Medium $\underline{Q}$ is the incident medium. Both the exit and incident media are assumed to be semi-infinite. The layer index, $i$, goes from 1 to $m+1$. Each layer $i$ has a corresponding thickness, $z_{j}$, and complex index of refraction, $n_{j}$. The interfaces between the layers are assumed to be abrupt plane parallel and infinite in lateral extent. Each layer is assumed to be homogeneous and isotropic.

An electromagnetic wave with wave vector, $\vec{k}_{0}$. incident onto a multilayer with only two interfaces is depicted in Figure 9. The subscripts of the wave vectors and fiela vectors indicate the layer number, the superscript $(t)$ indicates that the wave is progressing in the positive z direction (transmitted), and the superscript (r) indicates that the wave is moving in the negative z direction (reflected). The superscript $(t)$ indicates that the value of the fields should be taken on the side 


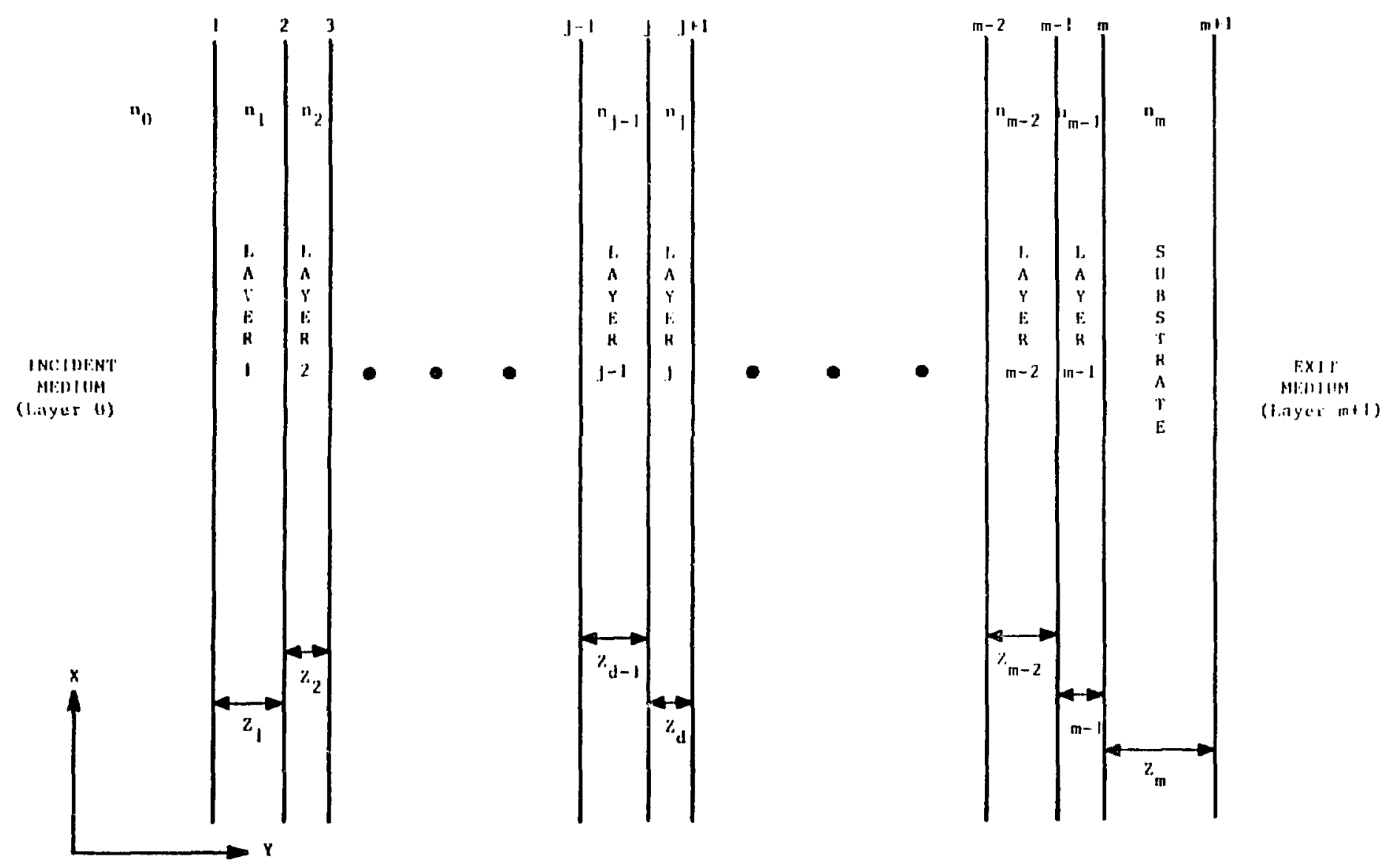

Figure 8. Schematic of an m layer multilayer showing labeling conventions for the layers and interfaces. 


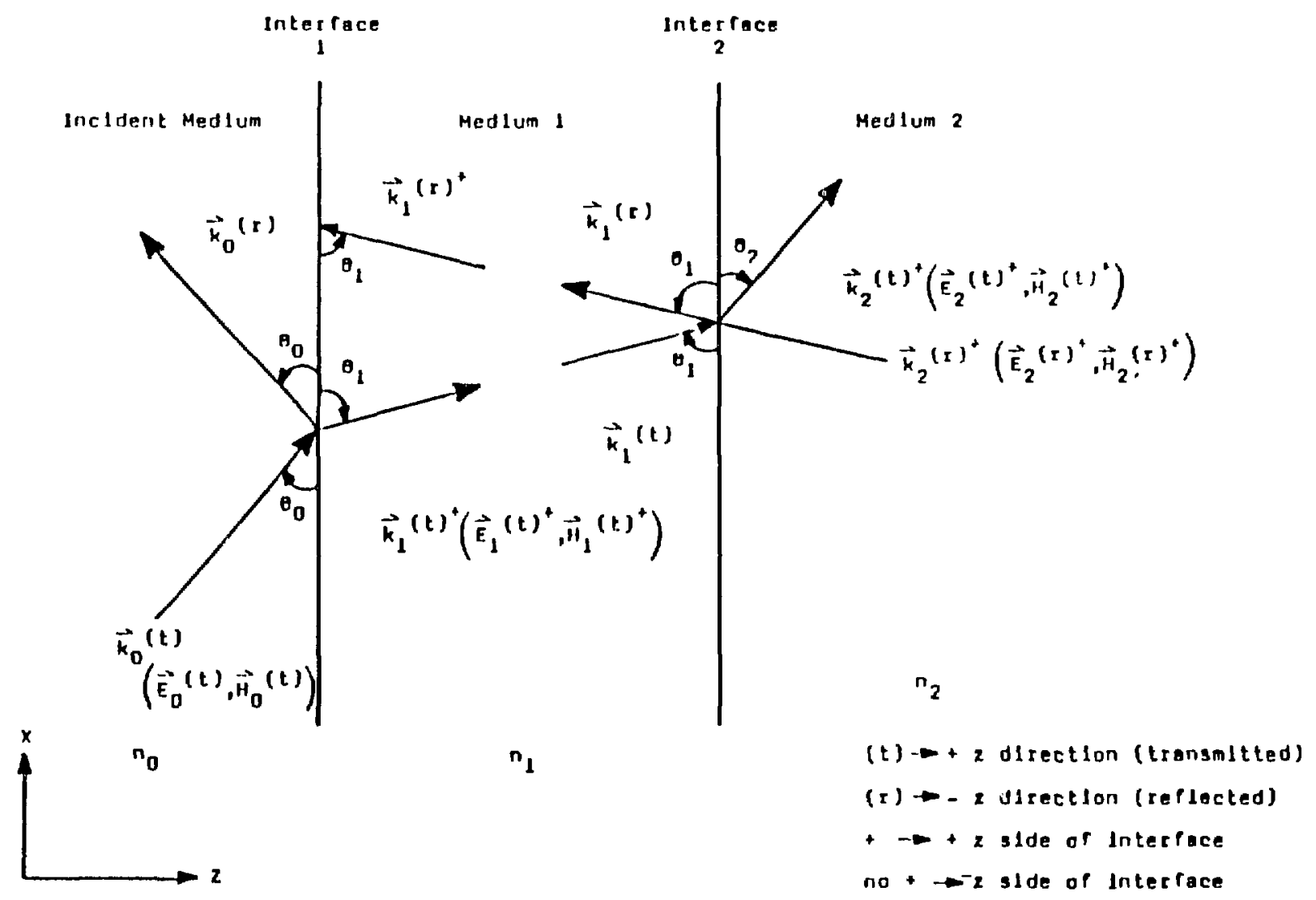

Figure 9. Schematic deplcting electric and magnetic field vectors at the first two interfaces of a multilayer system. 
of the interface of increasing $\underline{z}$, while the absence of a (t) indicates that the wave is to be taken on the side of the interface of decreasing $z$. All angles follow the $x-$ ray convention of being measured from the surface instead of the optical convention of being measured from the normal.

The basis of the electromagnetic boundary-value problem approach is the electromagnetic continuity equations at an interface between two layers. Boundary conditions across an interface that are derived from Maxwell's equations require the normal components of the magnetic field, 브, to be continuous and reguire the tangential component of the electric field, E, to bo continuous across an interface. For the normal incidence case these conditions for the first interface of Figure 9 can be written as

$$
\begin{aligned}
& E_{0}^{(t)}+E_{0}^{(r)}=E_{1}^{(t)+}+E_{1}^{(r)+} \text { and } \\
& H_{0}^{(t)}-H_{0}^{(I)}=H_{1}^{(t)+}-H_{1}^{(I)+} .
\end{aligned}
$$

The magnetic field amplitude is related to the electric field amplitude by the magnitude of its corresponding wave vector and thus to the index of refraction of the corresponding medium. Equation ( $5 b)$ can therefore be rewritten for nonmagnetic materials as

$$
n_{0} E_{0}^{(t)}-n_{0} E_{0}^{(I)}=n_{1} E_{1}^{(t)+}-n_{1} E_{1}^{(I)+} \text {. }
$$


It is also known that the field amplitudes at both interfaces inside the first layer are related to each other by the phase factors exp(ik, $\left.z_{1}\right)$ and $\exp \left(-i k_{1} z_{1}\right)$ since the wave travels through a distance of $z_{1}$ from ons interface to the other. That is,

$E_{l}^{(t)+}=E_{l}^{(t)} \exp \left(i k_{1} z_{1}\right)$, and

$E_{l}^{(I)+}=E_{l}^{(r)} \exp \left(-i k_{1} z_{1}\right)$.

The basic recursion relations between the electric field amplitudes on the $=z$ side of the first interface with that on the $\underline{-z}$ side of the next interface are determined by combining the sets of Eqs. (5) and (6). This yields the relations $E_{0}^{(t)}=\frac{1}{2}\left(1+\frac{\left.n_{1}\right)}{n_{0}} E_{1}^{(t)} \exp \left(i k_{1} z_{1}\right)+\frac{1}{2}\left(1-\frac{\left.n_{1}\right)}{n_{0}} E_{1}^{(r)} \exp \left(-i k_{1} z_{1}\right)\right.\right.$, and $E_{0}^{(r)}=\frac{1}{2}\left(1-\frac{\left.n_{1}\right)}{n_{0}} E_{1}^{(t)} \exp \left(i k_{1} z_{1}\right)+\frac{1}{2}\left(1+\frac{\left.n_{1}\right)}{n_{0}} E_{1}^{(r)} \exp \left(-i k_{1} z_{1}\right)\right.\right.$, Equations ( $7 a)$ and ( $7 b$ ) can be generalized for any succeeding two interfaces in a multilayer by replacing the o with $j-1$ and the 1 with 1 , giving

$$
\begin{aligned}
& E_{j-1}^{(t)}=\frac{1}{2}\left(1+\frac{\left.n_{j}\right)}{n_{j-1}} E_{j}^{(t)} \exp \left(i k_{j} z_{j}\right)+\right. \\
& \quad+\frac{1}{2}\left(1-\frac{n_{j}}{n_{j-1}}\right) E_{j}^{(r)} \exp \left(-i k_{j} z_{j}\right) \text { and }
\end{aligned}
$$




$$
\begin{gathered}
E_{j-1}^{(r)}=\frac{1}{2}\left(1-\frac{n_{j}}{n_{j-1}}\right) E_{j}^{(t)} \exp \left(i k_{j} z_{j}\right) \\
\quad+\frac{1}{2}\left(1+\frac{\left.n_{j}\right) E_{j}^{(r)}}{n_{j-1}} \exp \left(-i k_{j} z_{j}\right)\right.
\end{gathered}
$$

The basic recursion relations ( $8 a$ ) and ( $8 b$ ) can be generalized to oblique angles of incidence by replacing $\underline{n_{j}}$ with $\eta_{j}$ where

$$
\begin{aligned}
& \eta_{j}=\frac{n_{j}}{\sin \theta_{j}} \quad(\text { p polarization }) \text { and } \\
& \eta_{j}=n_{j} \sin \theta_{j} \text { (E polarization) } .
\end{aligned}
$$

The electric field vector for p polarization is parallel to the plane of incidence and is perpendicular to the plane of incidence for 5 polarization. The angles, $\theta_{j}$, are grazing angles that can be complex and are related to the incident angle $\theta_{0}$ by the equation

$$
\theta_{j}=\sin ^{-1}\left(1-\frac{n_{0}^{2} \sin ^{2} \theta_{0}}{n_{j}^{2}}\right)^{x} \text {. }
$$

For oblique angles of incidence, following Berning (1963) the quantity $\mathrm{kj}_{j} \mathrm{z}_{j}$ becomes the effective phase thickness, I' of the layer; that is,

$k_{j} z_{j}+v_{j}=\frac{2 \pi}{\lambda} n_{j}{ } i n \theta_{j} z_{j}$.

Equations ( $8 a$ ) and ( $8 b$ ) then become for the general angle case 


$$
\begin{aligned}
E_{j-1}^{(t)}=\frac{1}{2}(1+ & \frac{\left.\eta_{j}\right)}{\eta_{j-1}} E_{j}^{(t)} \exp \left(i \psi_{j}\right) \\
& +\frac{1}{2}\left(1-\frac{\left.\eta_{j}\right) E_{j}^{(r)}}{\eta_{j-1}} \exp \left(-i \psi_{j}\right)\right.
\end{aligned}
$$

End

$$
\begin{aligned}
& E_{j-1}^{(r)}=\frac{1}{2}\left(1-\frac{\left.\eta_{j}\right)}{\eta_{j-1}} E_{j}^{(t)} \exp \left(i \varphi_{j}\right)\right. \\
& \quad+\frac{1}{2}\left(1+\frac{\left.\eta_{j}\right)}{\eta_{j-1}} E_{j}^{(r)} \exp \left(-i \Psi_{j}\right) .\right.
\end{aligned}
$$

The reflectivity, $\underline{R}$, and transmission, $\underline{T}$, for a multilayer are then given by $R=\left|\frac{E_{0}^{(r)}}{E_{0}^{(t)}}\right| 2$ and $T=\frac{\operatorname{Real}\left[\hat{n}_{m+1}\right]}{n_{0}}\left|\frac{E_{m+1}^{(t)+}}{E_{0}^{(t)}}\right|^{2}$.

The recursion relations, Eqs. ( $12 \mathrm{a}$ ) and (12b), are applied successively. starting from the substrate-exit medium interface with the initial condition that $\mathrm{E}_{m}^{(r)+}=0$ until the field amplitudes in the incident medium are calculated.

The matrix formulism arises from putting Egs. (12a) and ( $12 \mathrm{~b}$ ) into matrix form.

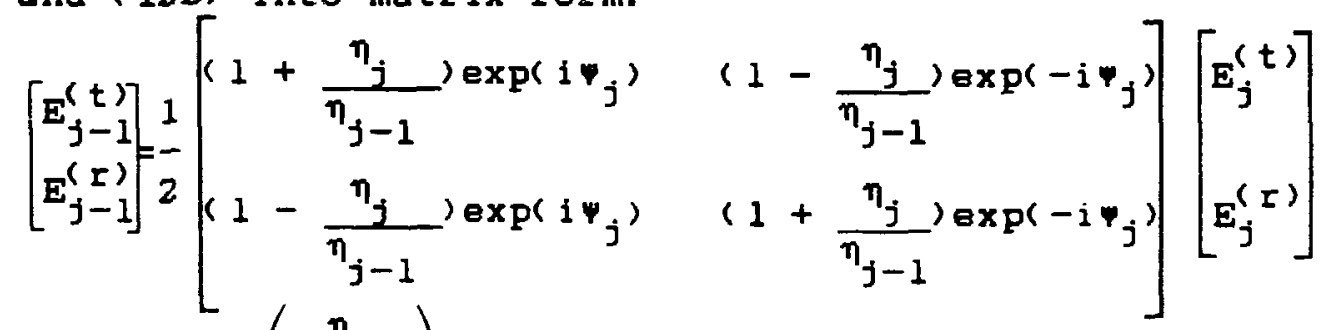

Factoring $\left(1+\frac{\eta_{j}}{\eta_{j-1}}\right)$ out of the above equation gives 
$\left[\begin{array}{l}E_{j-1}^{(t)} \\ E_{j-1}^{(r)}\end{array}\right]=\frac{1}{1-r_{j-1, j}}\left[\begin{array}{lr}\exp \left(i \psi_{j}\right) & r_{j-1, j} \exp \left(-i \psi_{j}\right) \\ r_{j-1, j} \exp \left(i \psi_{j .}\right) & \exp \left(-i \psi_{j}\right)\end{array}\right]\left[\begin{array}{l}E_{j}^{(t)} \\ E_{j}^{(r)}\end{array}\right]$

where $I_{j-1, j}$ is the Fresnel reflection coefficient for either $\mathrm{p}$ or $\mathrm{E}$ polarization, given by

$\Sigma_{j-1, j}=\frac{\eta_{j-1}-\eta_{j}}{\eta_{j-1}+\eta_{j}}$.

Equation (14) can be written in short notation as $\left[\begin{array}{c}E_{j-1}^{(t)} \\ E_{j-1}^{(r)}\end{array}\right]=M_{j}\left[\begin{array}{l}E_{j}^{(t)} \\ E_{j}^{(r)}\end{array}\right]$.

Applying equation (16) $\underline{m+1}$ times gives

$\left[\begin{array}{c}E_{0}^{(t)} \\ E_{0}^{(I)}\end{array}\right]=\left[\begin{array}{c}E_{m+1}^{(t)} \\ E_{m+1}^{(\tau)}\end{array}\right]=M_{1} M_{2} M_{3} \cdots M_{m} M_{m+1}\left[\begin{array}{c}E_{m+1}^{(t)} \\ E_{m+1}^{(r)}\end{array}\right]$.

and the reflectivity and transmission are given by

$R=\left|\frac{M_{21}}{M_{11}}\right|^{2}$ and $T=\frac{\left.\text { Real[ } n_{m+1}\right]}{n_{0}}\left|\frac{1 .}{M_{11}}\right|^{2}$.

If the multilayer is periodic, then the matrix describing the period is the product of the matrices making up the period. Because of the nature of these matrices, the resultant matriy is unimodular. If $E$ is the matrix that describes a period, and if there are $\underline{N}$ periods, then their resultant product (Born and wolf, 1983) can be given as

$$
\begin{aligned}
& \mathbf{P}=\left[\begin{array}{ll}
P_{11} & P_{12} \\
P_{21} & P_{22}
\end{array}\right] \quad \text {, and } \\
& \mathbf{P}^{N}=\left[\begin{array}{ll}
P_{11} U_{N-1}(a)-U_{N-2}(a) & P_{12} U_{N-1}(a) \\
P_{21} U_{N-1}(a) & P_{22} U_{N-1}(a)-U_{N-2}(a)
\end{array}\right],(19)
\end{aligned}
$$


where

$a=x_{2}\left(P_{11}+P_{22}\right)$ and $U_{N}(x)=\frac{\sin \left[(N+1) \cos ^{-1} x\right]}{\sqrt{1-x^{2}}}$.

The $\underline{U}_{N}$ are Chebyshev polynomials, and when the multilayer is periodic, their use saves computation.

The matrix formulism of Lee (1981) and others is equivalent to Eq. (16). The recursion relation used by Underwood and Barbee ( 198 la and b) can be similarly derived as Egs. ( $12 a)$ and ( $12 b)$; however, they take the point at which to reference the recursion relation to be in the center of the layer instead of at the interface.

The advantages of the optical multilayer method are that the Chebyshev polynomial product allows quick computations of periodic systems, each layer in the multilayer can be unique, non-repeating roughness and transition layers can be incorporated, the effects of the substrate can be included, and the absorption within any particular layer can be readily calculated.

The main purpose of this thesis is to investigate the changes in the reflection properties of miltilayers subjected to a harsh radiation environment. Under such conditions the multilayer is expected to absorb more radiation in its upper layers, producing physical changes in these layers. For this reason, it was necessary to use a method that would allow each layer to be unique, 
and it was determined that the matrix method was the best method to use in this application. For room temperature evaluation of the multilayers, the Modifled Darwin--Prins model was used in addition to the matrix method.

\section{Data Base}

For each of these methods, it is necessary to know the following multilayer propertles:

1) layer composition

2) physical thickness of the layers

3) complex Index of refraction or the atomic scattering factors.

If the physical thickness and material composition of the multilayer are known, then the approprlate optical constants must be determined. The atomic scattering factors are taken from the literature and are the key components of a data base necessary for any of the theoretical calculations.

The index of refraction is related to the atomic scattering factor of the composite material by the equation

$n=1-6-i \beta$,

where

$\delta=\frac{r_{0} \lambda^{2}}{2 x} N \overline{f_{1}}$ and $\beta=\frac{r_{0} \lambda^{2} N f_{2}}{2 \pi}$. 
Here $\overline{f_{1}}$ and $\overline{f_{2}}$ are the average atomic scattering factors, $r_{0}$ is the classical electron radius, 스 15 the incident wavelength, and $\underline{N}$ is the number of molecules per unit volume. The data base is bullt on the tables of atomic scattering factors for elements of atmic number from 1 to 94 for energies of $100 \mathrm{eV}$ to 2 keV (Henke et al.. 1982) that have been recently extended to 10 keV (Auerbach and Tirsell, 1985). Since the time-resolved spectral information of the plasma source used in this investigation could be obtained down to $25 \mathrm{eV}$, it was necessary to extend the compliation down to 25 ev for the elements of interest: tungeten, carbon, aluminum, silicon, and molybdenum. The procedure used to extend the compilation for each element is described below.

Carbon. Carbon can exist in many forms, and its optical properties are extremely preparation-dependent. The research group at Deutches Elektronen-Synchrotron (DESY) (Hagemann, Gudat, and Kunz, 1974) have compiled the real and imaginary parts of the dielectric constant for evaporated thin films of carbon with a reported density of $1.5 \mathrm{~g} / \mathrm{cm}^{3}$. The $\underline{f}_{1}$ and $\underline{f}_{2}$ values are related to the dielectric constant by the equations

$$
\begin{aligned}
& f_{1}=\frac{\pi}{r_{0} N \lambda^{2}} \epsilon_{1} \text { and } \\
& f_{2}=\frac{\pi}{r_{0} N^{2}} \epsilon_{2} .
\end{aligned}
$$


These values were used to determine the $\underline{f}_{1}$ and $\underline{f_{2}}$ values below $100 \mathrm{eV}$. For the $\underline{f}_{2}$ data, it was necessary to scale the DESY data by $2.0 / 1.5$ to be continuous with the Henke data at $\sim 100 \mathrm{eV}$. This ratio is also the difference in the reported densities of the carbon films that the two investigators used. Likewise, for the $\underline{f}_{2}$ values derived from the DESY data it was necessary to scale them by 1. $/ 1.04$ to coincide with the Henke data.

Tungsten. Values for the real and imaginary parts of the index of refraction of tungsten taken from Lynch and Hunter (1985) were used to determine the atomic scattering factors between $25 \mathrm{eV}$ and $47 \mathrm{eV}$ using an assumed density of $19.3 \mathrm{~g} / \mathrm{cm}^{3}$. Because their scattering data ended at $47 \mathrm{eV}$ and Henke's compilation staried at $\sim 100 \mathrm{eV}, \quad a$ straight-line interpolation between the two data sets was taken for the $\underline{f_{1}}$ values. For the $\underline{f_{2}}$ values. Henke's compilation included points to $30.5 \mathrm{eV}$, and the calculated $\underline{f_{2}}$ values from Lynch and Hunter (1985) were scaled to match Henke's data at that point.

Silicon. Silicon $\underline{f_{1}}$ and $\underline{f_{2}}$ scattering factore were needed to account for the silicon substrate. The real and imaginary parts of the complex index of refraction were taken from Edwards (1985). A density of $2.26 \mathrm{~g} / \mathrm{cm}^{3}$ was assumed. Both the calculated $\underline{f}_{1}$ and ${\underline{f_{2}}}_{2}$ values were scaled by a factor of 0.97 to match the Henke data at 
$\sim 100 \mathrm{eV}$.

Molybdenum. Molybdenum-carbon multilayers were also investigated; therefore, scattering factors for molybdenum were needed. The real and imaginary parts of the index of refraction were taken from Weaver, Krafka, Lynch, and Koch $(1981)$ to calculate the $\underline{1}_{1}$ and ${\underline{f_{2}}}_{2}$ values, using an assumed density of $10.2 \mathrm{~g} / \mathrm{cm}^{3}$ for the range of 25-40 eV. The $\underline{f}_{2}$ values were scaled by 0.974 to match the Henke compilation at $30.5 \mathrm{eV}$. The $\underline{f}_{1}$ values were scaled such that the $40 \mathrm{eV}$ point intercepted a line determined by the first three data points in the Henke compliation. The $f_{1}$ scaling factor was 2.8 .

Aluminum. Aluminum $f_{2}$ scattering factors were needed to calculate the attenuation in the aluminum filters used. Both the ${\underline{f_{1}}}_{1}$ and ${\underline{f_{2}}}_{2}$ values were extended. The real and imaginary parts of the complex dielectric constant were taken from Hagemann et al. (1974), and the corresponding scattering factors were calculated using a density of $2.7 \mathrm{~g} / \mathrm{cm}^{3}$. The resulting $\underline{f}_{2}$ values were then scaled by 0.875 to march the Henke data point at $114 \mathrm{eV}$. The $f_{1}$ values were scaled by 0.958 to match the Henks data at $\sim 100 \mathrm{eV}$.

\section{Boundary Layer Roughness}

As pointed out earlier in the introduction, the 
layers in manufactured multilayers exhibit interfacial roughness, transition layers between two layers of different materials, and other imperfections. To calculate the actual x-ray response of a multilayer, these effects ought to be taken into account.

The effect of roughness on the scattering of visible and ultraviolet light from a surface has been extensively studied (Bennent and Bennent, 1969), and both scalar and vector theories have been developed (Beckmann and Spizzichino, 1963: Porteus, 1964). These theories have been applied to dielectric multilayers (Eastman. 1978; Carniglia, 1979; Elson, et al,. 1983) and metal multilayers (Rosenbluth and Forsyth, 1981).

A straightforward way to incorporate roughness into the multilayer calculations is to use the matrix method (Carniglia, 1979). This allows each layer to have different characteristics if necessary and simplifies bookkeeping.

A very simple way to account for the degradation of the reflection peak due to roughness is to use a DebyeWaller factor (Barbee, 1981). This method assumes that the roughness at the interfaces is random and exhibits a Gaussian distribution. The difference in the measured reflection peak and the calculated peak without roughness is related to the roughness of the interfaces by the 
equation $\frac{P(\text { measured })}{P(\text { calculated })}=\exp \left[-\left(\frac{4 \pi \Delta z}{\lambda} \sin \theta\right)^{2}\right]=\exp \left[-\left(2 \ln \frac{\Delta z}{d}\right)^{2}\right]$

where $\underline{A 2}$ is the RMS roughness of the interface, $\theta$ is the incident angle. $\underline{\lambda}$ is the incident wavelength, $\underline{n}$ is the order of diffraction, and $d$ is the d-spacing of the multilayer.

Optical profilometer measurements were taken on multilayer oVLA-130B-2 (tungsten-carbon). A series of seven measurements across the multilayer was averaged, and a 5.77-8 RMS roughness over a length of $700 \mu_{m}$ was obtained. An example of the optical profilometer data for this multilayer is shown in Figure 10. Dr. B. L. Henke and his staff at Lawrence Berkeley Laboratory measured this multilayer at four diffraction orders for Al $K_{\alpha}$ radiation and two orders for $C u L_{\alpha}$ radiation. These measured peak reflectivity values are given in Table 1. Also in Table 1 are the peak reflectivity values calculated by the matrix method using a $\Gamma=0.37$ and a $\underline{2 \mathrm{~d}}=135 \mathrm{R}$ for the tungsten-carbon multilayer. The last column of Table $l$ gives the resulting 쓰 values derived from Eq. (20). The average value of the roughness for these $x-r a y$ measurements is $5.5 \& \pm 1.0 \&$, which is in very good agreement with what was observed by optical profilometry. 


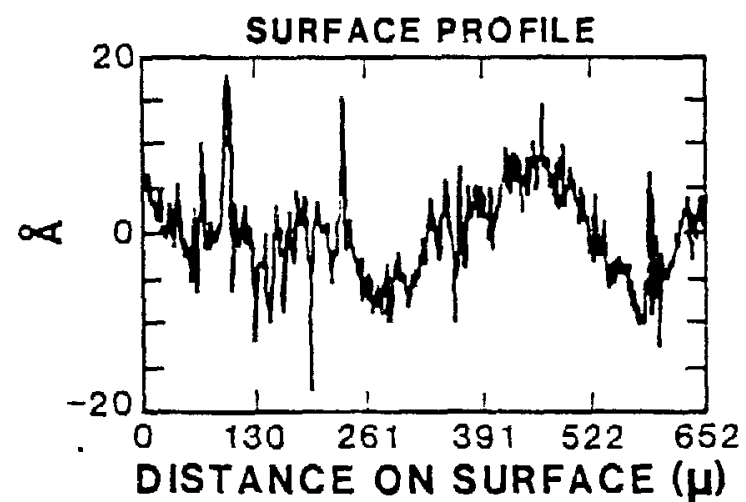

HISTOGRAM OF SURFACE HEIGHTS
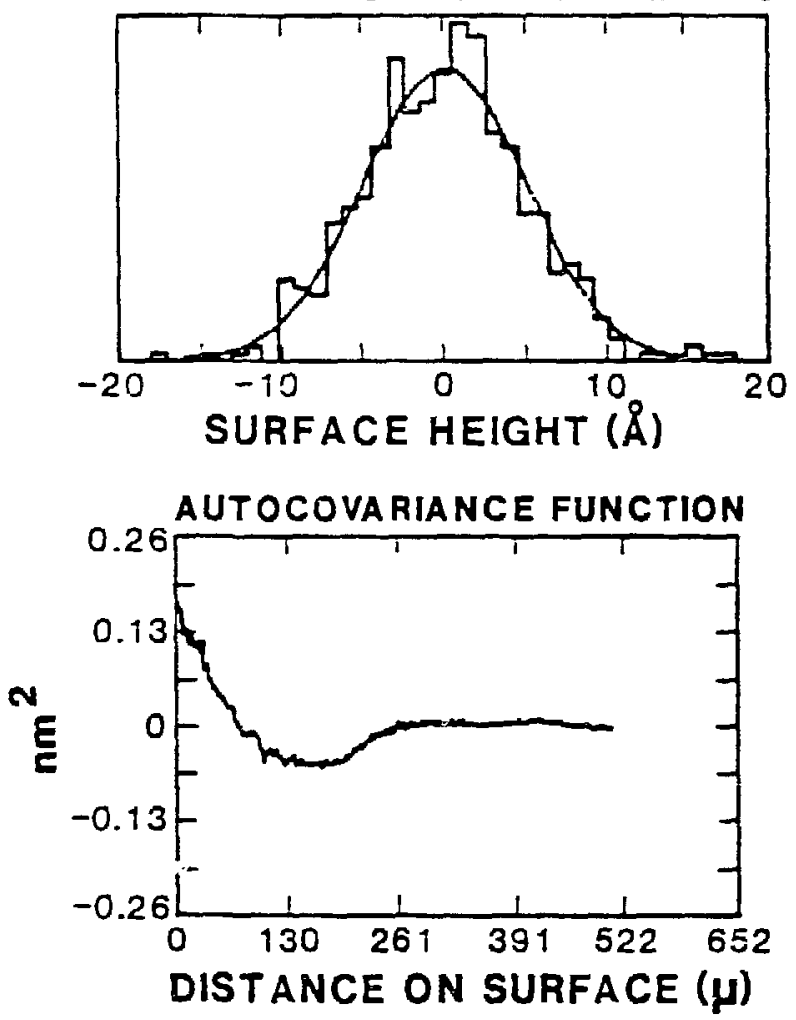

Figure 10. Example of the roughness data obtained with optical profilometry for multilayer OVLA-13CB-2. Information of the surface profile, the distribution of surface heights, and the autocovariance function assuming a Gaussian distribution of surface heights is obtained. 
TABLE 1. MEASUREMENTS ON OVLA-13OB-2

\begin{tabular}{lcccc}
\hline Energy & n & $\begin{array}{c}\text { Measured } \\
P(8)\end{array}$ & $\begin{array}{c}\text { Calculated } \\
P(\%)\end{array}$ & $\begin{array}{c}\Delta z \\
(8)\end{array}$ \\
\hline 1486.7 & 1 & 34.4 & 53.6 & 7.2 \\
1486.7 & 2 & 6.5 & 15.7 & 5.1 \\
1486.7 & 3 & 0.13 & 1.1 & 5.2 \\
1486.7 & 4 & 0.10 & 5.3 & 5.4 \\
929.7 & 1 & 29.0 & 39.2 & 5.9 \\
929.7 & 2 & 4.8 & 8.5 & 4.0 \\
\hline
\end{tabular}


SUMMARY PART I

In Chapter I-1 the $x-r a y$ and physical characteristics of metal multilayer x-ray diffractor elements were reviewed. Heasurement techniques of the $x-$ ray characteristics, both as a function of energy and as a function of angle, were presented. The set of measurements as a function of angle at $C u L_{\alpha}$ wavelength for the multilayers investigated in this experiment can be found in Appendix A.

In Chapter I-2 the different methods for computing the $x$-ray properties were presented. Justification was given as to the choice of the thin-film matrix method for this investigation. The data base that allows calculation of the optical properties of the materials in the multilayers was discussed and a beief discussion of interfacial roughness presented. By taking into account tine interfacial roughness with a Debye-haller factor, the calculated values of the peak reflection were shown to match the experimental data. 
PART II

MULTILAYER CHARACTERIZATION UNDER INTENSE FLASH

X-RAY LOADING 
CHAPTER II-1

THE SOURCE AND ITS CAPABILITIES

To investigate the $x-r a y$ reflectivity changes of interest in metal multilayers under intense flash-heat loading, a heating source was required that would meet the following criteria:

1) is intense enough to melt at least the top few layers of the multilayer,

2) provides heating throughout the layers in which $x$ ray diffraction takes place.

3) has $x-r a y$ line components or could be used in confunction with an $x$-ray source that provides $x-r a y$ line spectra that would permit measurement of the reflectivity of multilayers,

4) has a pulse duration of 10-50 nanoseconds,

5) has epectral characteristics that are known or measurable,

6) has a sufficisnt repetition rate to justify performing the experiment.

The time duration requirement and the intensity requirement eliminated the possibility of using conventional laboratory x-ray sources.

One promising source was a pulsed laser-produced plasma. For example, Kohler et al. (1978) used x rays 
produced from a lo-J Nd glass laser with a 10-ns pulse incident on a copper target to heat a potassium acid pthalate (KAP) crystal $1 \mathrm{~cm}$ from the target. Potassium acid pthalate has a higher ratio of mass absorption coefficient to heat capacity compared with that of a tungsten-carbon multilayer: therefore, heating a tungsten-carbon multilayer would require even greater laser energy output. For this reason, the 10-J laser then available would not be adequate. Additionally, the x-ray spectrum from a laser-produced plasma is hard to quantify, making modeling difficult. It is of interest to note that during the course of this investigation, Kohler et al. (1985) increased the output of their Naglass laser to $100 \mathrm{~J}$ and measured the heating of metal multilayers with the same experimental configuration as for their KAP experiments in the time-integrated mode. Their results were described qualitatively, and no modeling was attempted.

Another method considered for heating the multilayer was to use resistive heating, obtained by running a current through the multilayer and using an auxiliary $x$-ray source to probe the multilayer's reflectivity. This type of experiment could be done in a standard laboratory setting; however, relating the changes in the multilayer structure and reflectivity due 
to resistive heating to that due to $x-r a y$ heating was judged unnecessarily complicated. In addition, modeling would have been almost impossible since investigators have even had difficulty modeling the change of state of resistively heated planar single material foils (Logan et al., 1977).

Another possible $x$-ray source that could be used to do the heating as well as provide radiation components for probing multilayer reflection properties was a fast z-pinch. A-pinch is a machine that sends large amounts of current along its z-axis. The current induces a strong magnetic field around the z-axis, and the resulting Id $\vec{I} \mathrm{X}$ E force collapses the plasma along that axis. Fast Z-pinches produce high-intensity $x$ rays efficiently (Hammel and Jones, 1984; Spielman et al.. 1985).

A particular $\mathrm{Z}$-pinch machine that appeared useful was the recently upgraded accelerator. PROTO II, at Sandia National Laboratories in Albuquerque, New Mexico. of particular interest was its gas puff mode in which a cylindrical annulus of ionized low-density noble gas is imploded. By changing the noble gas, different lines in the kiloelectronvolt anergy region of the $x$-ray spectrum can be produced (Maxon and Wainwright, 1984; Spielman et al., 1985). These 1 ines in the kiloelectronvolt region 
of the spectrum can contain as much as 10 percent of the total radiation yield. Specifically, radiation from neon gas puffs looked very promising. A typical neon gas puff implosion on PROTO II produced $30 \mathrm{~kJ}$ of total radiation. of which 2-3 $\mathrm{kJ}$ were in the kiloelectronvolt spectral region. Of particular importance was the fact that the 2-3 kJ of kiloelectronvolt radiation was mostly confined to the hydrogen- and helium-like resonance lines at 1022 and $922 \mathrm{eV}$, as shown in Figure 11. Because of the low level of background and continuum in this region of the spectrum, these lines appeared suitable for probing the multilayer $x$-ray reflectivity characteristics.

There were two additional features of PROTO II that made it attractive. One was that it averages a shot a day and that a run of several months' duration had already been scheduled that would permit adequate experimentation. The second feature was that PROTO II already had an experimental team that was diagnosing the plasma characteristics. Since diagnostic ports were available on this machine and administrative arrangements were feasible for this collaboration, it was decided to field the experiment on PROTO II.

\section{PROTO II}

PROTO II (Johnson, 1976; Martin et al, 1976a and 


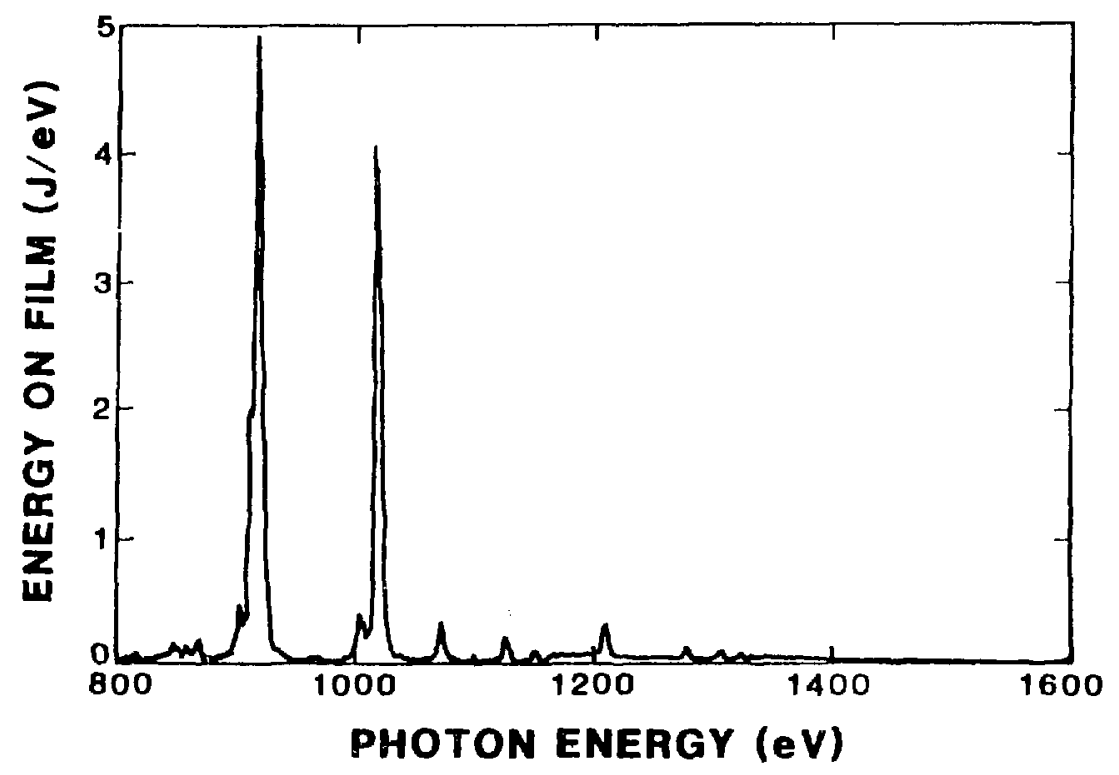

Figure 11. Time-integrated spectrum taken by $M$. A. Palmer of Sandia Laboratories with a potaseium acid pthalate curved-crystal spectrograph. 
1976b) is a multimodule water-insulated, pulsed power machine, A drawing of PROTO II is shown in Figure 12 . Eight $100 \mathrm{kV}$ oil-insulated Marx generators feed sixteen 7.5-nfd water-filled capacitors. The stored energy is switched through eight $\mathrm{SF}_{6}$ gas trigatron switches from the water capacitors to sixteen water-insulated lines. In each set of 1 ines the first pulse-forming line stores the energy from the capacitor, then a self-breakdown switch closes and charges the pulse-forming 1 ine in about 60 ns. The electrical energy is fed into the modified vacuum diode as shown in Figure 13. The hollow cylindrical gas annulus is injected into a 2.0-cm-long gap between the gas nozzle in the center of the cathode and a Ni wire mesh strung on stainless steel return current posts that are part of the anode. The energy flows into the diode in a fast $4 \times 10^{6}$-ampere pulge with a FWHM of 55 60 ns. The gas implosion $t$ ime, defined as the time between the arrival of the current pulse and the peak of the radiation pulse, is typically between 60-80 ns, depending on the nature and mass of the gas used.

The converging pulse 1 ines were approximately level with 8 radial diagnostic ports that exited at 75 degrees from the z-axis. These pulse lines were water-insulated, which means they were under some three feet of water. Any diagnostic that was used on these ports had to be 
Figure 12. An artist's conception of the PROTO II Zpinch, pulse-power machine. Drawing is courtesy of Rick Spielman. Sandia National Laboratories. 


\section{PROTO II}

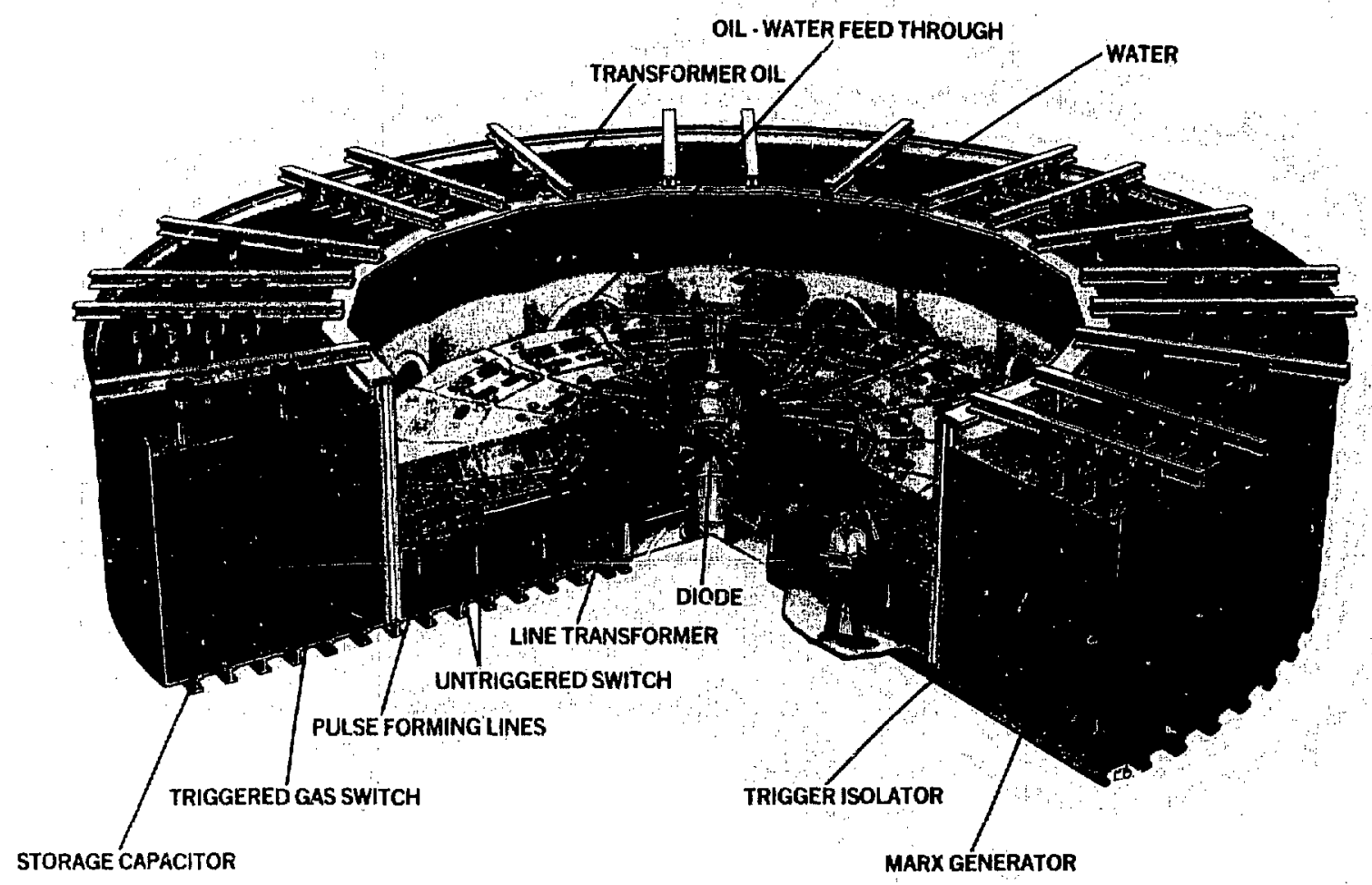




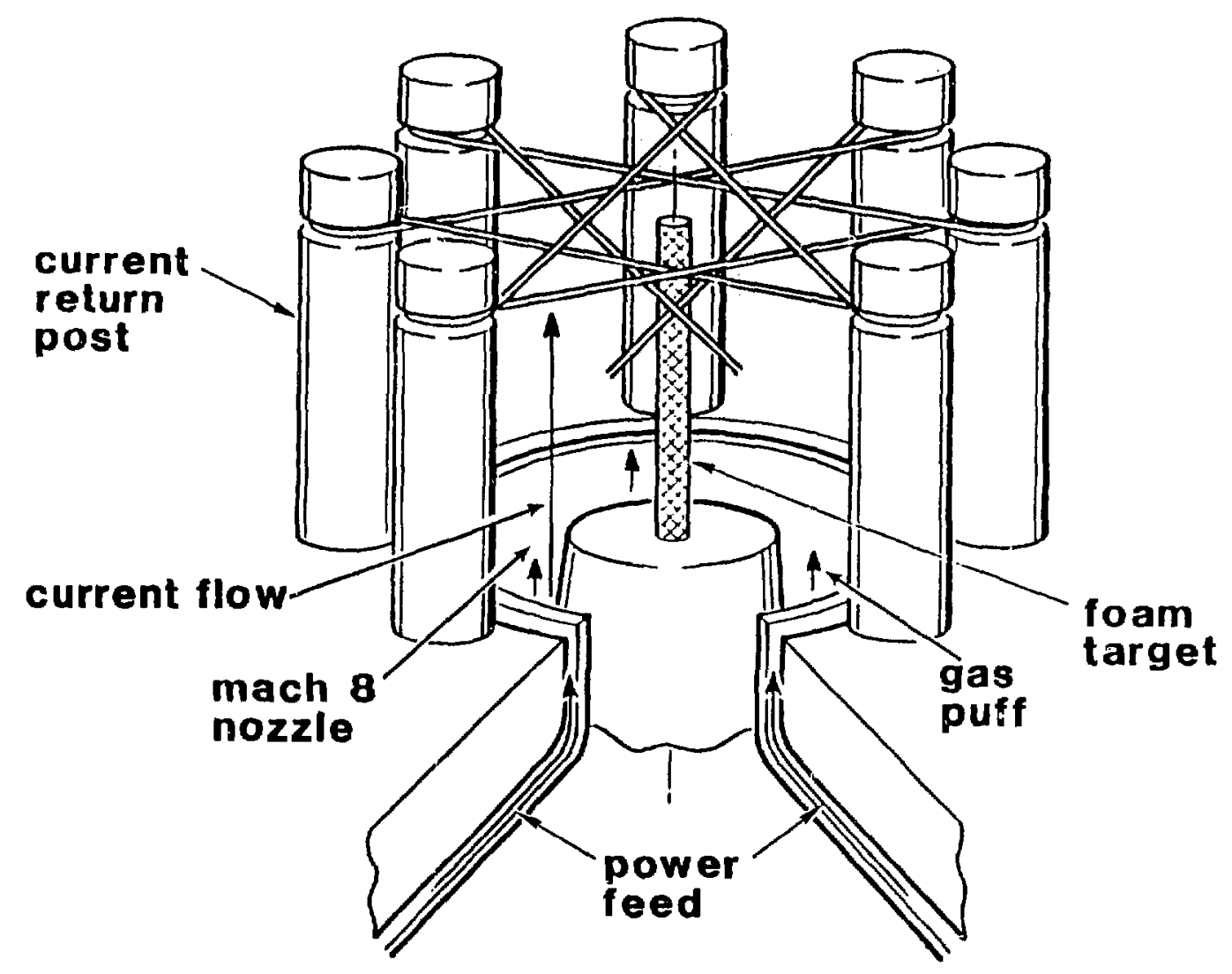

Figure 13. Schematic of the gas nozzle/target assembly showing the configurations of the target, nozzle, return current posts. and electrical feed. Drawing courtesy of Sandia National Laboratories. 
vacuum-compatible on the inside and water-compatible on the outside and could not rust or pollute the very clean water. Near axial ports on the machine were not immersed. Additional precautions had to be taken when designing the experiment. For example, apparatuses that wəre attached to the machine had to be more than a certain minimum distance from the current-carrying lines to ensure against electrical arcing.

\section{Sandia Diaqnostics}

The experimental team of PROTO II fields a variety of time-integrated and time-resolved diagnostics for each shot. Data from these are used to characterize the plasma Eource. Time-integrated diagnostics include 2 or $3 x$-ray pinhole cameras, a curved-crystal KAP spectrograph, and a grazing incidence $x$-ray spectrograph. The major $t$ imeresolved diagnostics of interest that are fielded are 6 $x$-ray diodes (XRD's) and 2 thin-film bolometers.

\section{Time-Integrated Diagnostice}

X-ray pinhole cameras. Quant itative information on the size of the plasma and the location from which energy was emitted inside the plasma was obtained from $x$-ray photographs taken with pinhole cameras. The $x-r a y$ pinhole cameras were fielded by M. A. Palmer and A. Widman. 
Photographs with different energy sensitivities are obtained in two ways. One way that spectral differentiation can be obtained is by using a film pack with several different films in a stack. The first film's energy response is its inherent $x$-ray response (Henke et al., $1984 \mathrm{a}$ and $\mathrm{b})$, convolved with the response of the prefilter. The first piece of film acts as a filter for the second piece of film; therefore, the second film's energy response is the corvolution of the transmission of the prefilter and the first piece of film and the $x-r a y$ response of the second piece of film. Succeeding film responses are calculated in a similar manner.

The film packs used on PROTO II contained two pieces of film. The films were RAR 2497 and SB-5. in that order. The energy response of the stack of films convolved with a $12.7-\mu_{m}$ beryllium prefilter transmittance is shown in Figure 14. The first piece of film gives an indication of where $x$ rays $\geq l$ keV are beirg emitted from the plasma, while the second piece of film indicates the source distribution of $x$ rays of $22.2 \mathrm{kgV}$. A sample photograph of an image taken on the first piece of $f$ ilm is shown in Figure 15 .

The second way spectral differentiation is obtained is by using the critical angle characteristics of $x$-ray mirrors to cut out the high-energy portion of the spec- 


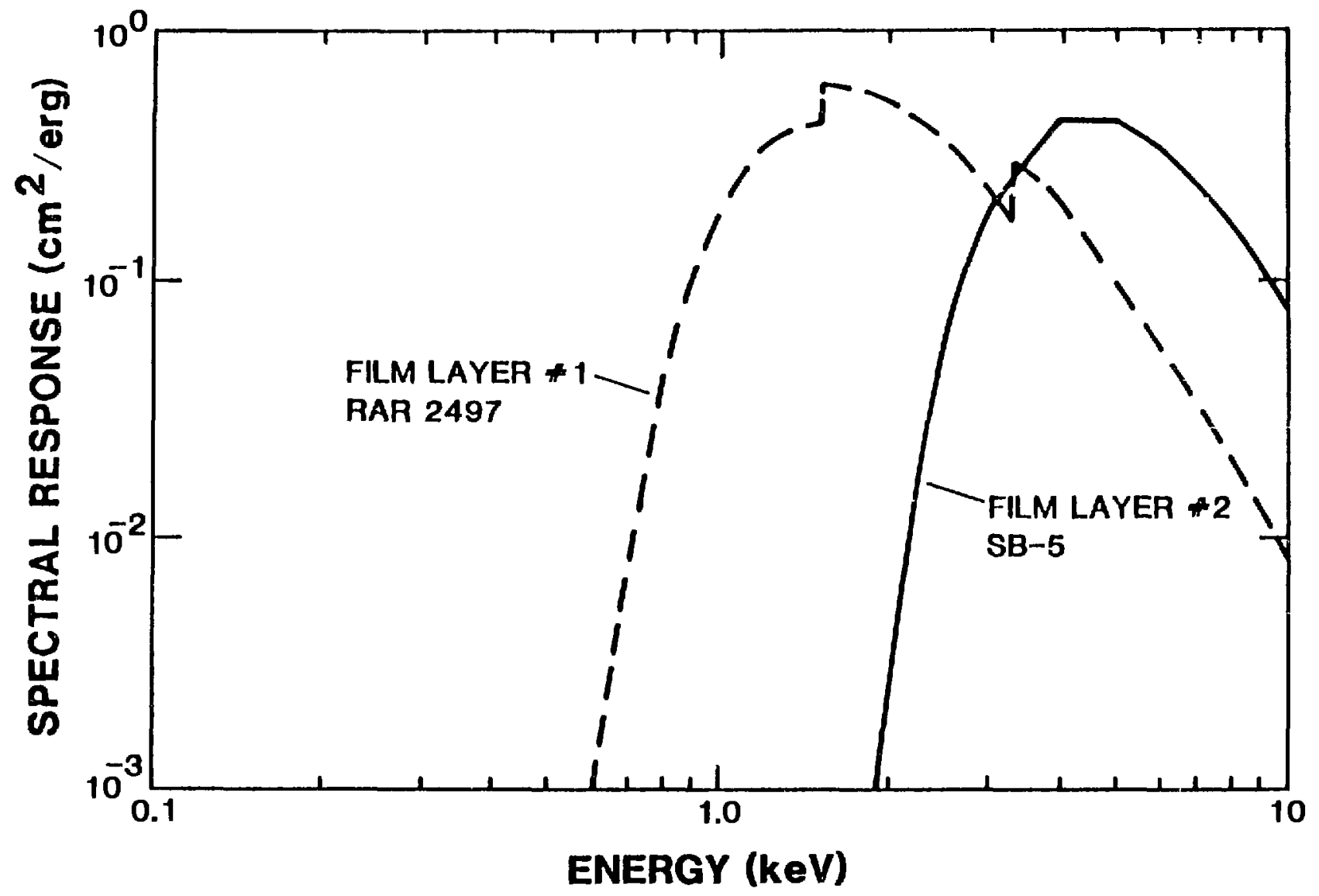

Figure 14. Spectral responee of the two-layer film pack for the $x-r a y$ pinhole cameras fielded by M. A. Palmar. 
Figure 15. Example of a kiloelectronvolt pinhole image of the PROTO II source. 


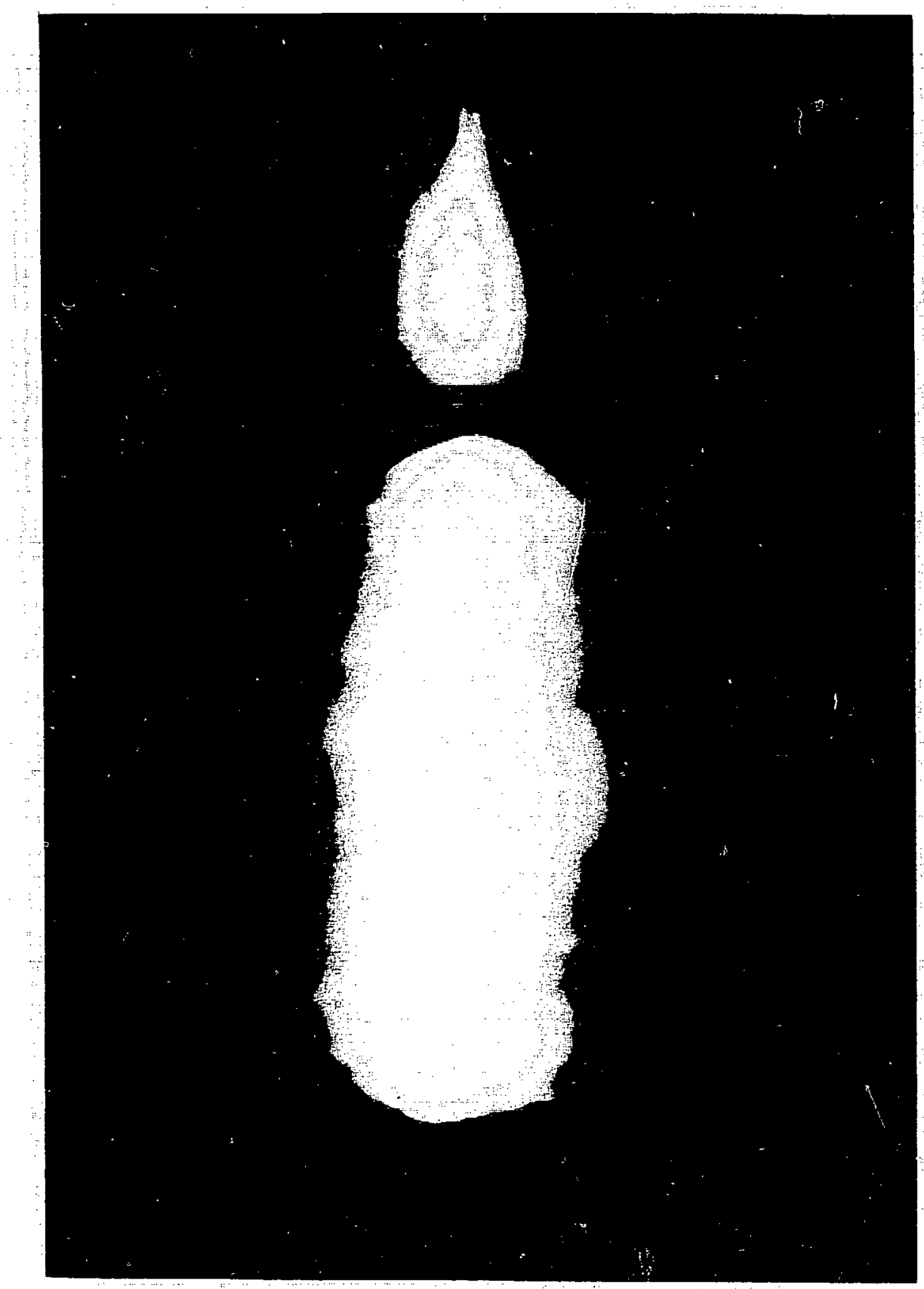


trum. This low-energy spectral imaging is obtained by csing two carbon mirrors at $3.72^{\circ}$ grazing incidence after the pinhole to keep photons with energies higher than 284 eV from being incident on the $x$-ray film. A 2000- $\AA$ gold filter is used as a prefilter, and the $x-r a y+i l m$ is RAR 2497. The sensitivity as a function of energy for the carbon mirror channel is shown in Figure 16. This pinhole camera records an image of the plasma in the 100284 eV spectral range.

In summary, images from the pinhole photographs gave information about the source in the spectral ranges from 100-284 eV, ? $1 \mathrm{keV}$, and $\geq 2.2 \mathrm{keV}$.

Potassium acid pthalate curved-erystal spectrograph. The KAP spectrograph consists of a convex-curved KAP crystal of radius of curvature $2.5 \mathrm{~cm}$ and a nonconcentric curved-film plane of radius of curvature 8.3 $\mathrm{cm}$. The spectrograph is usually fielded with a $12.7-\mu_{\mathrm{m}}$ beryllium entrance window and RAR $2497 \mathrm{film}$. In this configuration, it gives time-integrated spectral information in the range from $0.8 \mathrm{ksV}$ to $\leq 3 \mathrm{keV}$. An example of the type of spectral information obtained with this instrument is shown in Figure 11 . For the present experiments this instrument was fielded by the team of $M$. A. Palmer, A. Widman, and B. A. Hammel.

Grazing incidence spectroqraph. The grazing 


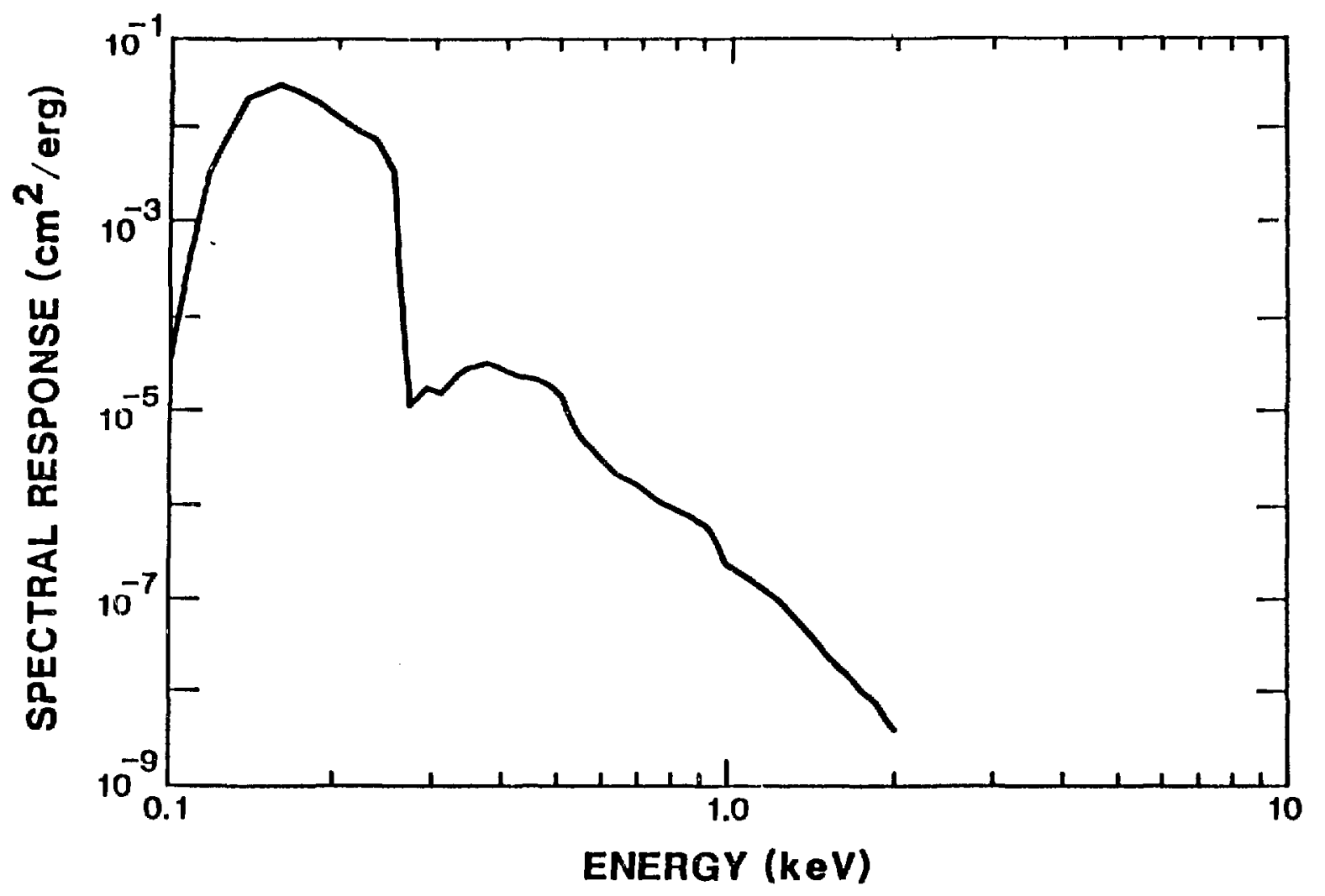

Figure 16. Absolute spectral sensitivity of the carbon mirror pinhole camera. Tranemiesion of the 2000-8 gold filter has been taken into account. 
incidence spectrograph gives qualitative spectral information in the range $20 \AA$ to $200 \AA$, using a 1200grooves/mm gold-coated grating at $88^{\circ}$ angle of incidence from the normal with a $10-\mu_{m}$ entrance slit. The detection film used is Kodak 101-06. The reason that these data are qualitative is that spectral sensitivity of films at grazing incidence is uncertain. Again, this instrument was fielded by the team of M. A. Palmer, A. Wic man, and B. A. Hammel.

\section{Time-Resolved Diagnostics}

x-ray diodes. Temporal spectral information in specific energy bands is obtained with XRD's. An XRD consists of a filter, a grid, and a photocathode. A positive voltage is applied between the grid and the photocathode. The grid collects the electrons produced by the photoelectric effect when $x$ rays are incident upon the photocathode. The loss of electrons from the photocathode is registered as a current pulse. The time resolution of the detector is determined by the spacing between the grid and the photocathode. The transmission of the filter in conjunction with the photocathode's guantum efficiency determines the spectral window of the XRD.

Six XRD's were fielded by R. B. Spielman and S. F. 
Lopez for this experiment. Three of the XRD's were sensitive to $x$ rays with energies below 1 keV, and three were sensitive to $x$ rays at 1 kev and above.

The $x$-ray flux the plasma produces is of such intensity that in order to keep the XRD's that are sensitive to low-energy $x$ rays from saturating, the XRD's have to be either meters away or the flux must be cut down in some way that still preserves the low-energy component. Therefore, the three low-energy XRD's in these experiments were pinhole imaged. In other words, the source was viewed by the XRD's through a pinhole such that the image of the source was entirely within the detectors' effective area. The three low-energy XRD's had aluminum photocathodes and were all $162.56 \mathrm{~cm}$ from the source. The photocathode guantum efficiency was taken from Day et al. (198la and b). The Channel 1 XRD had a filter of $250 \mathrm{~nm}$ of parylene and observed the source through a 150Mm-diameter pinhole located halfway betwwen the source and the detector at a distance of $82.55 \mathrm{~cm}$. The Channel 2 XRD had a filter of $0.75-\mu_{m}$ aluminum and viewed the source through a 500-lim-diameter pinhole also located halfway between the source and the detector at a distance of $82.55 \mathrm{~cm}$. The Chennel 3 xRD had a filter of $2-\mu_{\mathrm{m}}$ Kimfol and viewed the source through a pinhole of $500-\mu_{m}-$ diameter. The $x$-ray responees of Channels. 1-3 are given 
in Figure 17 and include the effective solid angle of the detectors.

The thros high-energy XRD's used gold photocathodes, were located at $162.56 \mathrm{~cm}$ from the source, and had effective areas of 0.75 square centimeters. The Channel 4 XRD had a filter of $2-\mu_{\pi} z i n c$. The Channel 5 $X R D$ had a filter of $0.5-\mathrm{mil}$ saran and 2-mil Kapton, and the Channel $7 \mathrm{XRD}$. following the labeling convention of Spielman, had filters of $0.5-\mu_{m}$ aluminum and $2-\mu_{m} \mathrm{Kimfol}$. The $x$-ray responses of Channels 4-7 are shown in Figure 18 and include all geometrical factors.

Data obtained from these six XRD's can be used to determine the spectrum produced by the source as a function of time.

Thin-film bolometers. Time-resolved thin-film bolometers (Mix et al., 1981) were flelded in these experiments by D. L. Hanson to measure $x-r a y$ fluence. Total energy yields were measured with a bare-nickel bolometer, and the yield of the radiation in the 500-eV to 40-keV range was measured witn a beryllium-parylenefiltered gold bolometer. Response functions of the bolometers fielded are shown in Figure 19, The data from the bolometers gave energy yields for each shot. 


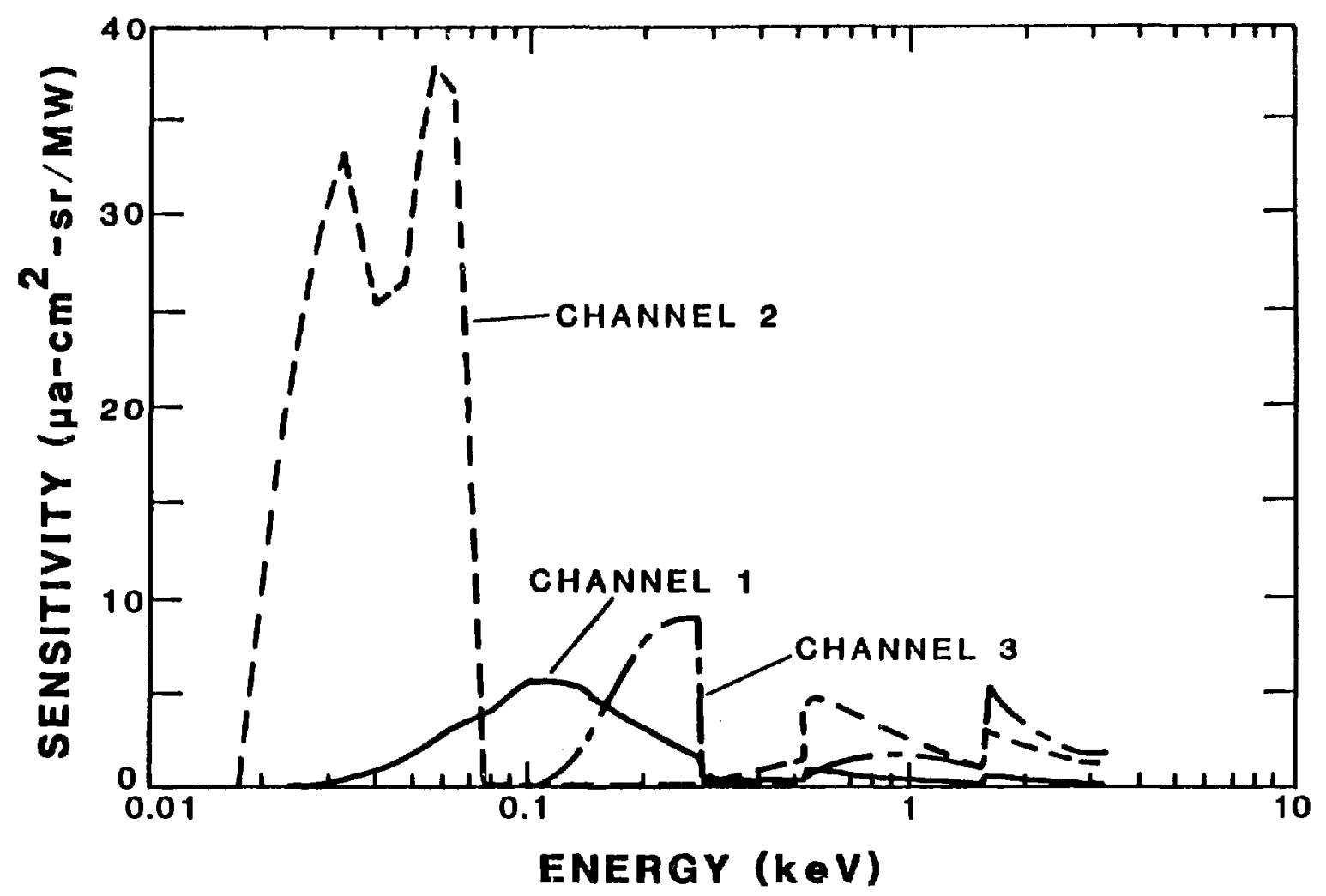

Figure 17. Spectral sensitivity of XRD Channels 1-3. assuming $1-\mathrm{cm}^{2}$ source and taking into account all geometrical factors. 


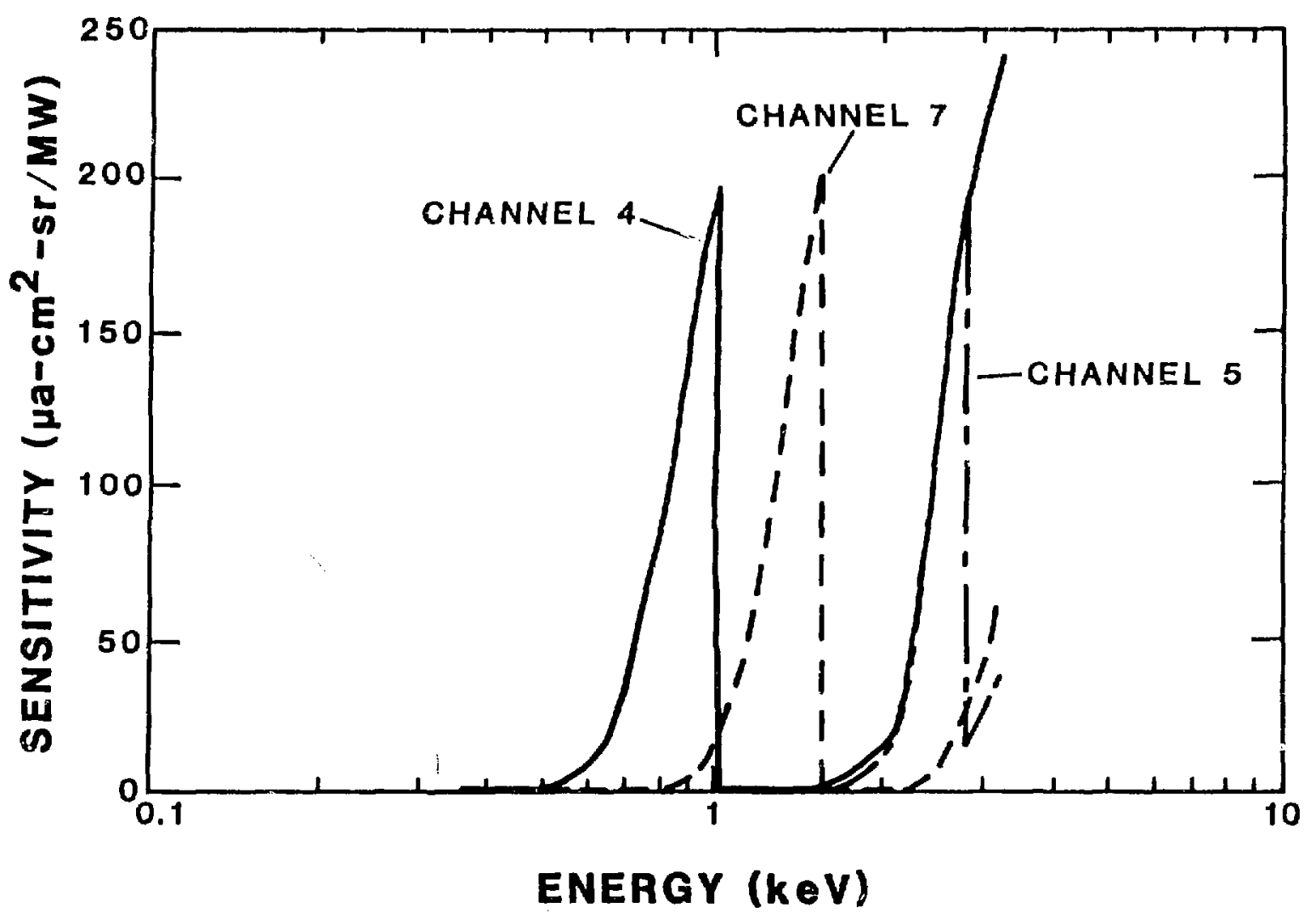

Figure 18. Spectral sepsitivity of XRD Channels 4, 5 , and 7, assuming a $1-\mathrm{cm}^{2}$ source and taking into account ald geometrical factors. 


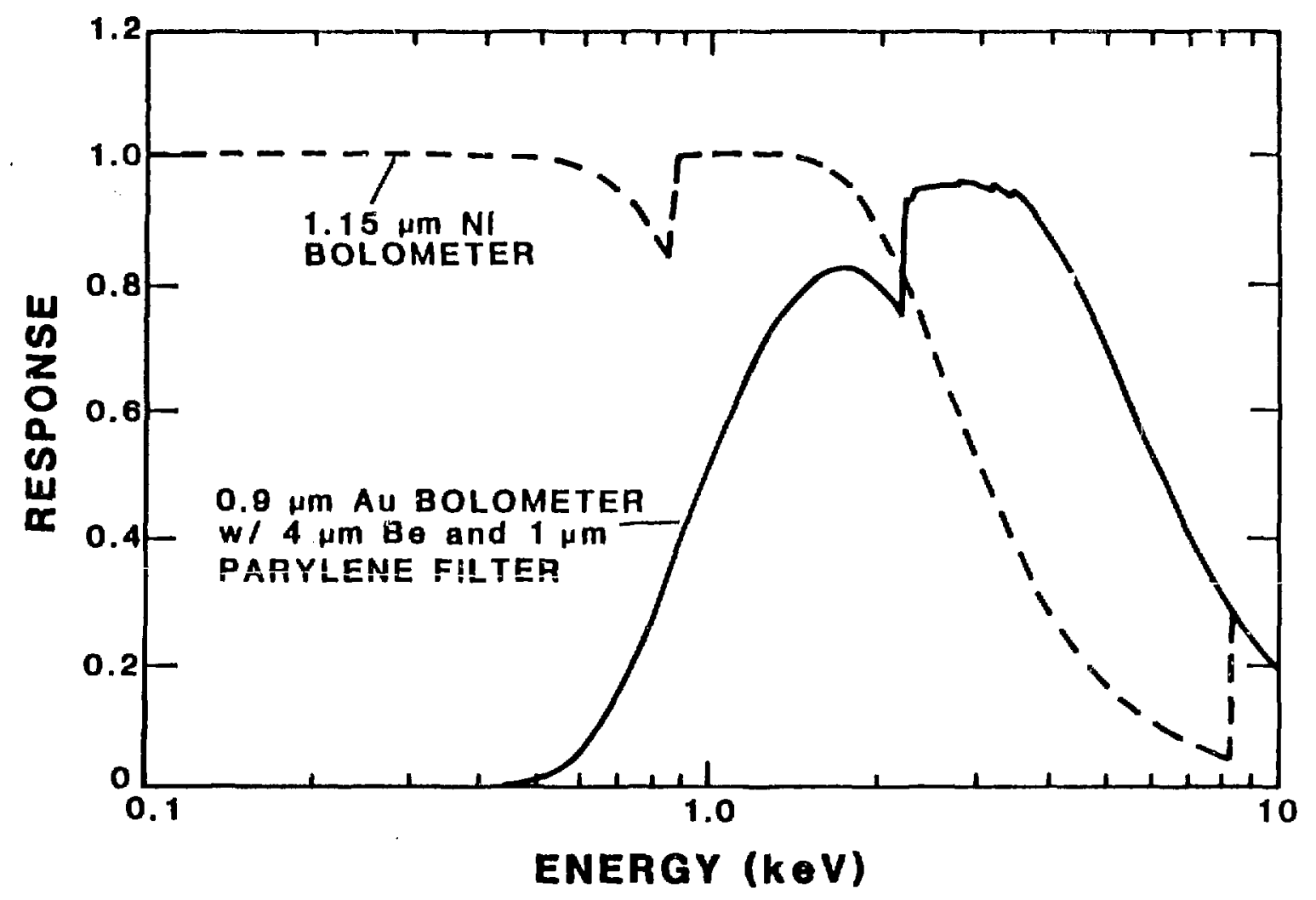

Figure 19. Spectral energy response of the two thin-film bolometers flelded by D. L. Hanson. 


\section{Rough Calculation of Heating Capabilties of PROTO II}

To determine whether the surface of a multilayer wili melt, the specific energy absorbed during a typical necn gas puff implosion can be calculated and compared with the enthalpy reeded for heating and melting the multilayer material. In a tungsten-carbon multilayer, the tungsten has a higher absorption coefficient than carbor and therefore will reach a higher temperature. The enthalpy needed to heat the tungsten through melting, $\Delta \mathrm{H}_{W^{\prime}}$ is given by the equation

$$
\begin{aligned}
\Delta \mathrm{H}_{W} & =H_{W}\left(T_{\text {melting }}\right)-H_{W}(2980 \mathrm{~K}) \\
& =552.2 \mathrm{~J} / \mathrm{g} .
\end{aligned}
$$

The specific energy absorbed, $\xi$, is given by the equation $\xi=\int_{0}^{t} \int_{0}^{\infty} \frac{d^{2} S}{d E d t} T_{f}(E) \mu_{W}(E)(1 .-R(E)) d E d t$.

where $\frac{d^{2} s}{d E d t}$ is the incident spectral flux in $\mathrm{W} / \mathrm{cm}^{2}-\mathrm{keV}$, $\underline{T}_{f}$ is the transmission function of any prefilter, $\underline{\mu}_{\omega}$ is the mass absorption of tungsten in $\mathrm{cm}^{2} / \mathrm{g}$, and $\underline{R}$ is the reflection coefficient for the multilayer stack. To determine whether PROTO II would be adequate, some understanding of the nature of the incident spectrum was needed.

If $29 \mathrm{~kJ}$ is a typical number for the total radiated energy and there are $2 \mathrm{~kJ}$ of $x$-ray $l$ ine radiation in a 
20-ns pulse, the spectrum can be approximated as consisting of a blackbody continuum and two lines at $1022 \mathrm{eV}$ and $922 \mathrm{eV}$ with $1 \mathrm{~kJ}$ total energy in each. The blackbody temperature can be calculated by taking the energy attributed to the continuum, $E_{c^{\prime}}$ and using the steianBoltzmann equation; thus,

$$
\begin{aligned}
& \pi B=\sigma T^{4} \frac{M W}{c m^{2}}, \text { and } \\
& \sigma T^{4}=\frac{E_{C}}{A_{s} \Delta t} .
\end{aligned}
$$

where $\underline{B}$ is the brightness, $\underline{\sigma}=5.67032 \times 10^{-8} \mathrm{~W} / \mathrm{m}^{2}-{ }^{a} \mathrm{~K}^{4}, A_{S}$ is the source surface ares, and $\underline{\Delta t}$ is the $x-r a y$ puise width. The source is taken to be a cylinder $2.0 \mathrm{~cm}$ long with a 2 -mm diameter, giving a surface area of 1.3194 $\mathrm{cm}^{2}$. To calculate $\mathrm{E}_{\mathrm{C}^{\prime}}$ the energy in the lines is subtracted from the totai energy, giving 27 kJ. Solving Eq. (23) for the average blackbody temperature, $I$, gives $I=\left(\frac{E_{C}}{A_{S} \Delta t \sigma}\right)^{*}$ : therefore.

$$
\begin{aligned}
T & =\left(\frac{27 \mathrm{~kJ} \times 1000 \mathrm{~J} / \mathrm{kJ}}{1.3194 \times 10^{-4} \mathrm{~m}^{2} \times 20 \times 10^{-9} \mathrm{~s} \times 5.6703 \times 10^{-8} \mathrm{~W} / \mathrm{m}^{20} \mathrm{~K}^{4}}\right) \\
& =6.5 \mathrm{I} 7 \times 10^{5}{ }^{\circ} \mathrm{K}=56.6 \mathrm{eV} .
\end{aligned}
$$

Using this temperature in Planck's law,

$$
B_{E}=\frac{d^{2} s}{d E d t}=\frac{(2 c)}{(h c)^{3}}\left(\frac{E^{3}}{e^{(E / k T)}-1 .}\right) \frac{M W}{c m^{2}-s r-k e V} .
$$


gives the average spectral distribution.

The complete spectral distribution is this blackbody spectrum plus the two lines in the kiloelectrosvalt region. From the spectrum shown in Figure 11 the half width at half maximum (HWHM) of the H-like resonance line was determined to be $10.1 \mathrm{eV}$, and the HWHM of the He-like resonance line was determined to be $8.3 \mathrm{eV}$. The line profils shape can be assumed for convenience to be Lorentzian, following the equation

$I_{1 i n e}(E)=\frac{1 .}{\pi H W H M} \frac{C_{1 i n e}}{\left[\frac{E-E_{1 i n}}{H W H M}\right]^{2}+1}$.

This equation, when integrated over energy, gives the coefficient $c_{\text {line }}$ to describe a line of $1-k J$ total energy. The appropriate coefficient is needed.

$C_{\text {ine }}=\frac{E_{\text {line }}}{4 \text { Kat } A_{5}}$.

The coefficient $C_{1 i n e}$ for a $1-k J, 20-n s$ pulse is therefore

$c_{\text {line }}=3.02 \times 10^{9}$

$\frac{J}{65-s-c m^{2}}$

Combining the corresponding distributions for the two lines and the blackbody continuum gives a representation of the incident spectrum shown in Figure 20 .

For a tungsten-carbon multilayer of $\underline{2 d}=100 \mathrm{~A}, \quad \Gamma=$ 


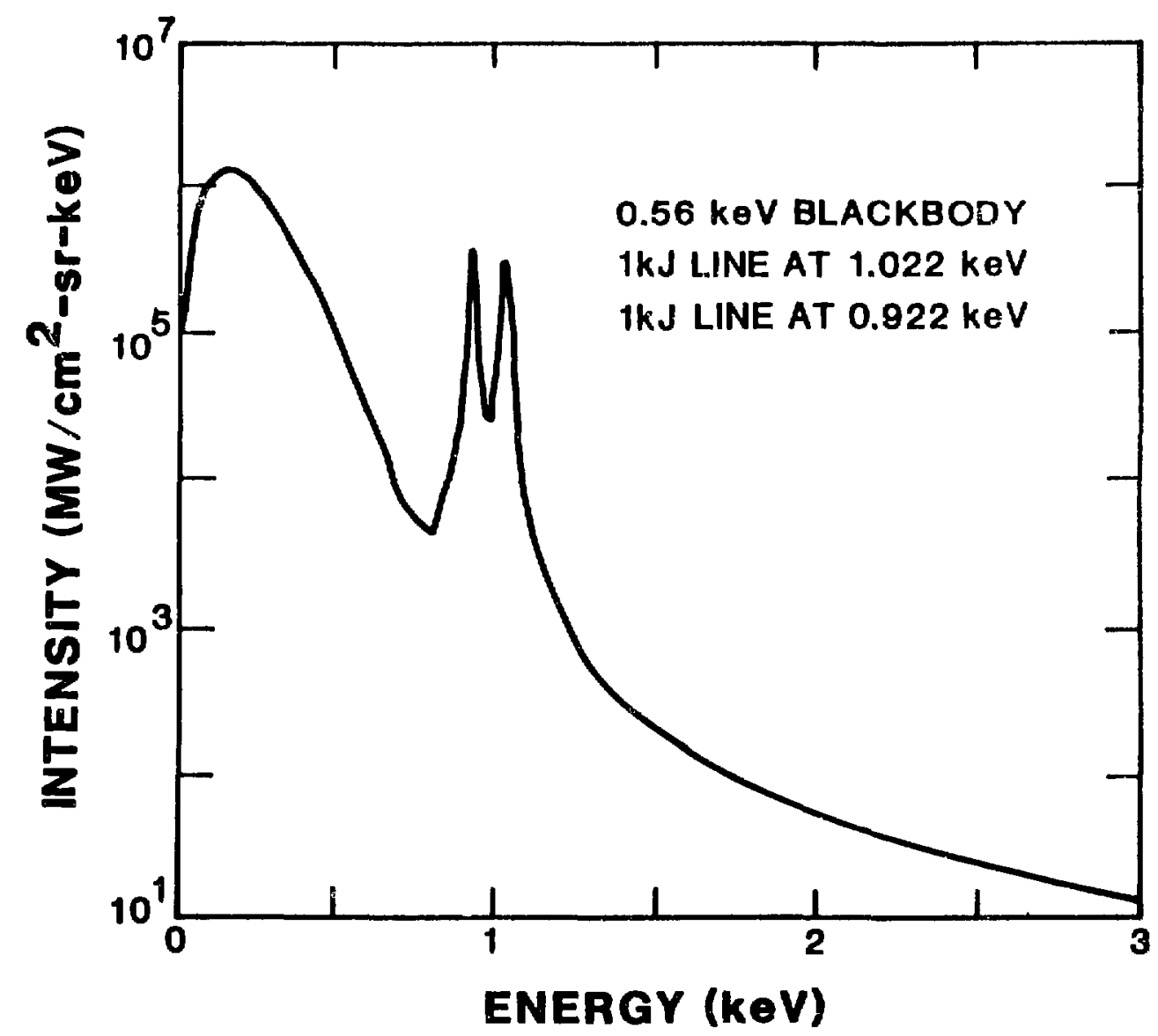

Figure 20. Bstimated spectra' shape for the $x$ rays produced by PROTO II. 
0.4. at an incident angle of 8.08 degrees, and with tungsten as the top layer, the calculated incident spectrum would heat the multilayer at $15 \mathrm{~cm}$ such that the first seven layers of tungsten would melt. A tungstencarbon multilayer of $\underline{2 \mathrm{~d}}=141 \mathrm{~A}$ and $\Gamma=0.43$ at 5 degrees incidence would get hot enough to melt the first 5 layers of tungsten. This calculation disregards the effects of conduction.

Because of the scale of the layers in the multilayer, thermal conductivity is very important and should be included in all detailed modeling that is done. This is illustrated by the following oversimplified discussion.

The sound speed of tungsten (Handbook of Chemistry and Physics, 1980) is such that a longitudinal wave could inake 890 round trips across a 30-A layer in one narosecond. Therefore, the layer could reach equilibrium in the 20-ns $x-r a y$ pulse. Since the tungsten layers absorb more radiation and heat up faster than the carbon layers, the cooler carbon layers will conduct heat away from the tungsten layers. The assumption is made that there is a $1^{\circ} \mathrm{K}$-temperature difference between two $30-8$ tungsten layers separated by a 40-8 carbon layer. Then, using a nominal thermal conductivity of $0.05 \mathrm{H} / \mathrm{cm}^{\circ} \mathrm{K}$ (Touloukian et a1.. 1970a) for the carbon layer, the amount of heat 
taken away is

$$
\begin{aligned}
q & =0.05 \frac{\omega}{\mathrm{cm}^{-0_{K}}} \times \frac{10_{K}}{40 \times 10^{-8} \mathrm{~cm}} \\
& =1.25 \times 10^{5} \frac{\mathrm{m}}{\mathrm{cm}^{2}}=5.22 \times 10^{5} \frac{\mathrm{cal}}{\mathrm{cm}^{2}-\mathrm{s}} .
\end{aligned}
$$

Dividing this number by the areal density of the carbon layer and multiplying by the $x$-ray pulse duration of 20 ns gives the total amount of heat taken away during the pulse $Q$$$
Q=\frac{5.22 \times 10 \times 20 \times 10^{-9}}{8.0 \times 10^{-7}}=1.3 \times 10^{4} \mathrm{cal} / \mathrm{g} .
$$

The amount of energy that was absorbed in the first tungsten layer was calculated from the above method to be only $\sim 6 \times 10^{4} \mathrm{cal} / \mathrm{g}$. Therefore, a temperature difference of only $4.6-{ }^{\circ} \mathrm{K}$ would allow all the heat of the tungsten layer to be cerried away. This means that conduction will definitely play a crucial role in the calculations. 
CHAPTER II-2

TIME-INTEGRATED EXPERIMENTS

The purpose of this phase of the investigation was to determine whether the overall experimental concept was sound. In adition, it allowed the author to become familiar with RROTO II and its physical constraints before designing the second phase of the investigation, the time-resolved experiments.

Two metal multilayer spectrometers were used in the experiments. Each spectrometer had provisions for a prefilter, a metal multilayer as the diffaction element, a postfilter, and a film pack as the detector. Spectrometer 1 was designed to have the metal multilayer diffraction element located $212 \mathrm{~cm}$ from the $x$-ray source. At this distance it would diffract the incident radiation without suffering physical damage due to radiation. Spectrometer 1 provided the "control" spectrum. spectrometer 2 was designed such that the metal multilayer diffraction element was only $15 \mathrm{~cm}$ from the plasma center where it could be expected to experience phjeical changes during the radiation pulge. These physical changes could be detected by comparing the difference in reflected spectra from the two spectrometers. Initial port assign- 
ments for this experiment placed Spectrometer 1 at a 13.5 degree port and spectrometer 2 at 75 degrees from the $\underline{-}$ axis. Both ports viewed the plasma from the same side. A general schematic of the two spectrometers is shown in Figure 21.

\section{Spectrometer 1}

A "safe" distance for the metal multilayer of Spectrometer 1 was determined to be $212 \mathrm{~cm}$ from "back of the envelope" calculations similar to those described in Chapter II-1. At this distance the multilayer would receive approximately $1 / 200$ the flux the metal multilayer at $15 \mathrm{~cm}$ from the source would receive. Originally. a port at 13.5 degrees from the z-axis was assigned for the use of Spectrometer 1. A distance of $212 \mathrm{~cm}$ would require a stainless steel pipe about 30 inches long. The distance from the detection plane to the metal multilayer was made as short as possible to minimize the possibility of mechanical interference with other experiments. A photograph of the components of Spectrometer 1 is shown in Figure 22. The manual valve was used as a shutterlight shield to protect the $x$-ray film during transit to and from PROTO II.

\section{Spectrometer 2}

The separations of the individual spectrometer 


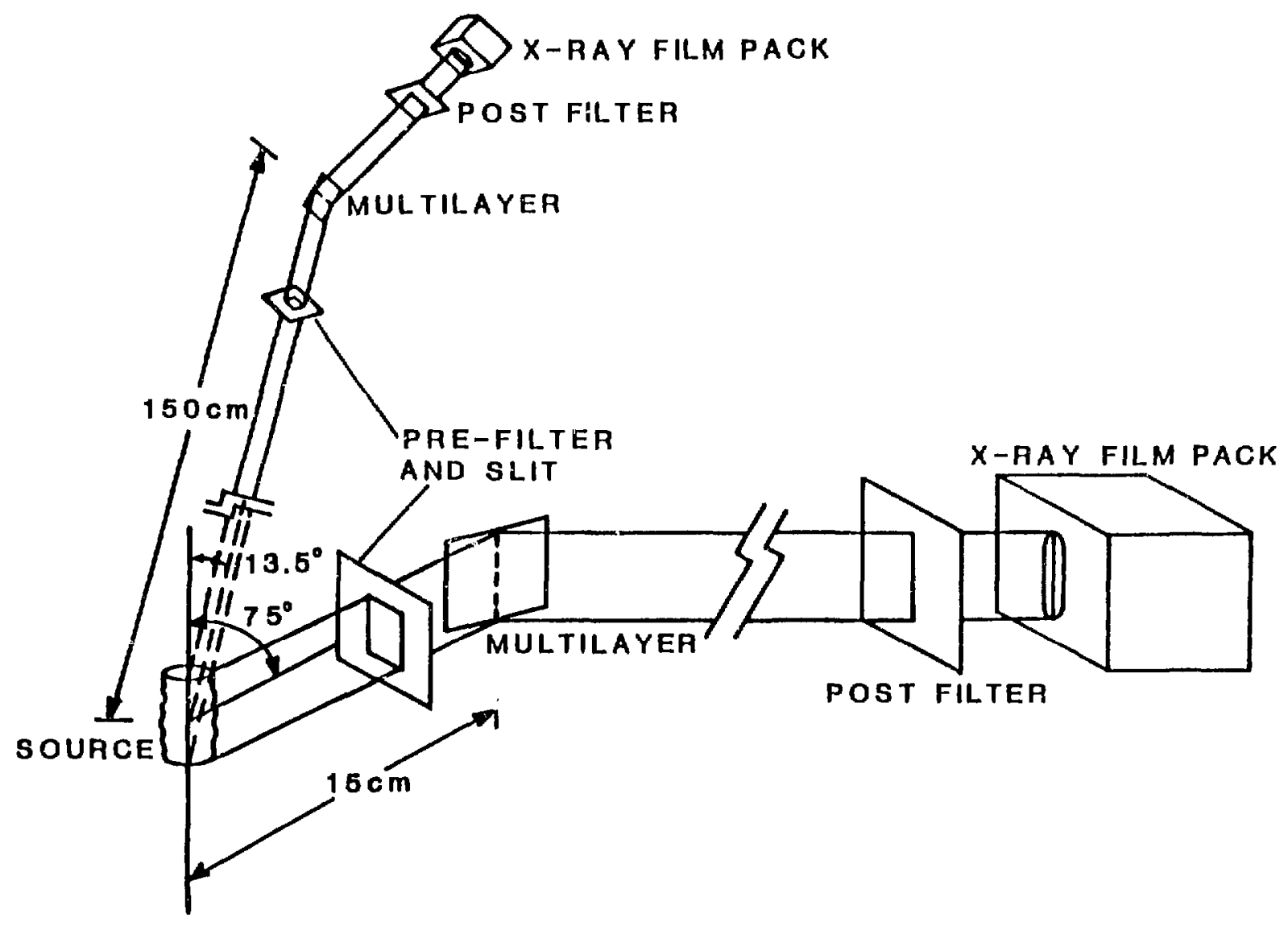

Figure 21. General schematic of the two spectrometers used at PROTO II. 
Figure 22. Photograph of the components of spectrometer 1 used in the time-integrated experiments. 


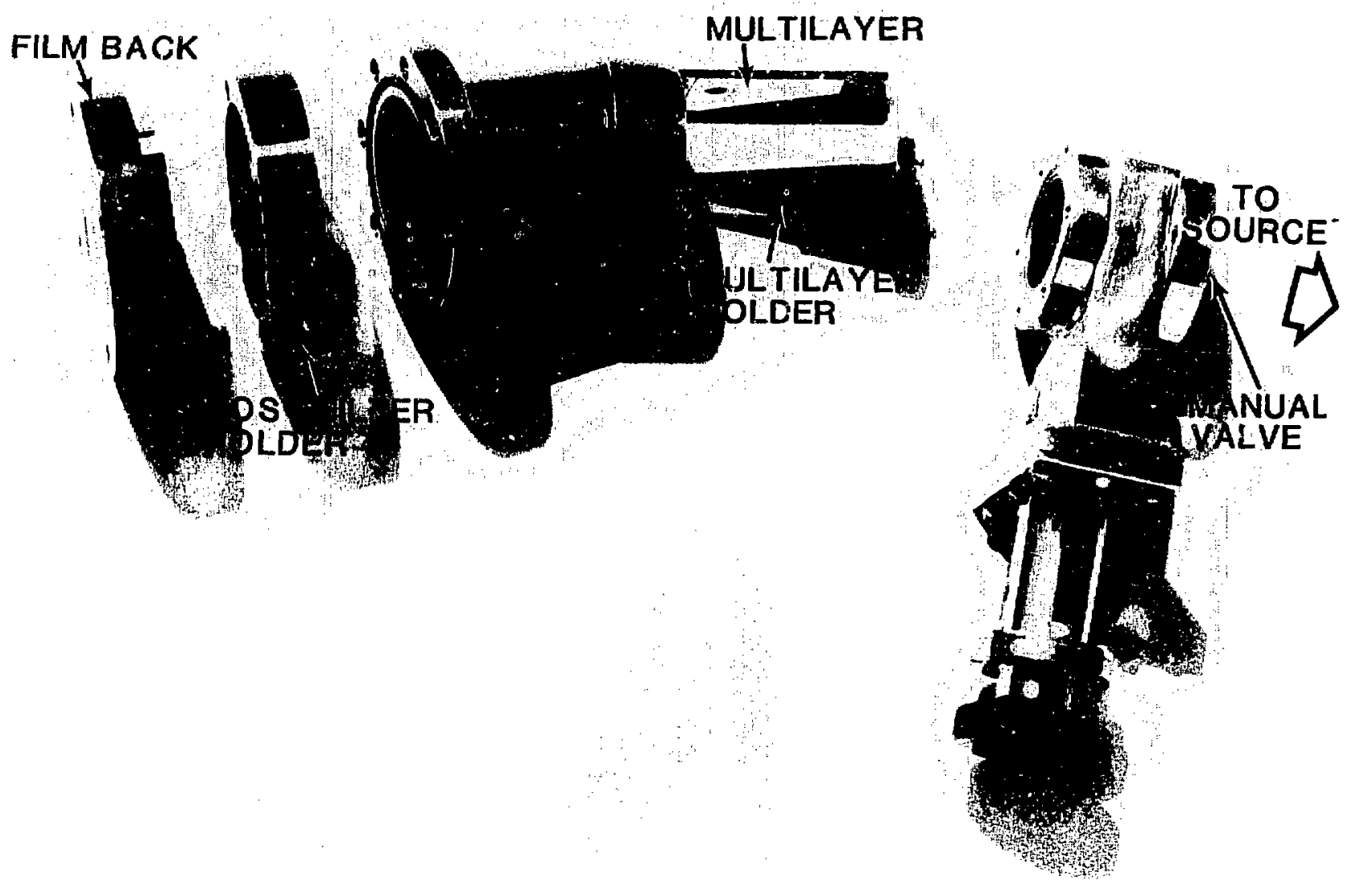


components of Spectrometer 2 were determined by the physical design of the existing vacuum system of PROTO II. The avallable space within the vacuum tank was $11 \mathrm{mited}$ and restricted. This was due to the fact that there were 8 radially positioned ports at 75 degrees from the z-axis. Outside the vacuum tank avallable space and positioning constraints were equally severe because of the placement and separation of the input-current feed lines around each port. These allowed placement of a 4.5-inch-diameter piece of apparatus no closer than $84 \mathrm{~cm}$ from the source outside the vacuum chamber.

Because of the physical constraints, the nominal Bragg angles of the metal multilayers had to be less than 11 degrees in order to prevent the multilayer placed 15 $\mathrm{cm}$ from the source from blocking other lines of sight and also to diffract the relevant $x$ rays out the available 75-degree port. The constraints on the Bragg angle set the possible 2a-spacing of the metal multilayers. Specifically, for $1 \mathrm{keV}$ radiation the 2d-apacing could not be less than $71.5 \&$. Since it had been determined that two crystal holders for nominal Bragg diffraction at 5 and 10 degrees would be used in tha spectrometers. the two multilayer 2d-spacings to be investigated were to be $\sim 70 \&$ and $\sim 140$ \&. Tungeten-carbon, molybdenumcarbon, ani palladium-carbon multilayers having the 
required 2a-spacings were obtained from Troy Barbee of Stanford University and were used as the Bragg diffraction elements in all the time-integrate experiments. The room temperature $x$-ray properties of these multilayers can be found in Tables $A 1$ and $A 2$ in Appendix $A$.

In order for multilayers of different d-spacings to view the source at the proper incident angles, a method for alignment and positioning the multilayers had to be developed. A method was evolved that used an alignment tube connected to a ball foint. The ball foint and alignment tube are shown in Figure 23. Assembly was done by sliding the alignment tube inside the vacuum flange with a slip ring holding the ball joint socket against the seating lip. The alignment tube could then pivot about the ball joint and was pointed with the alignment ring. The alignment ring was mounted on the inside of the PROTO II vacuum chamber. The multilayer and its holder were held in place at the other end of the alignment tube. A photograph of the components of spectrometer 2 is shown in Figure 23.

To align the spectrometer, a 2.54 cm-diameter portable jaser was directly coupled to the alignment tube. The laser beam ld reflect off the metal multilayer and would then shine into the vacuum chamber. By adjusting the position of the tube, the laser beam couid 
Figure 23. Photograph of the componente of Spectrometer 2 used in the $t$ ime-integrated experiments. 


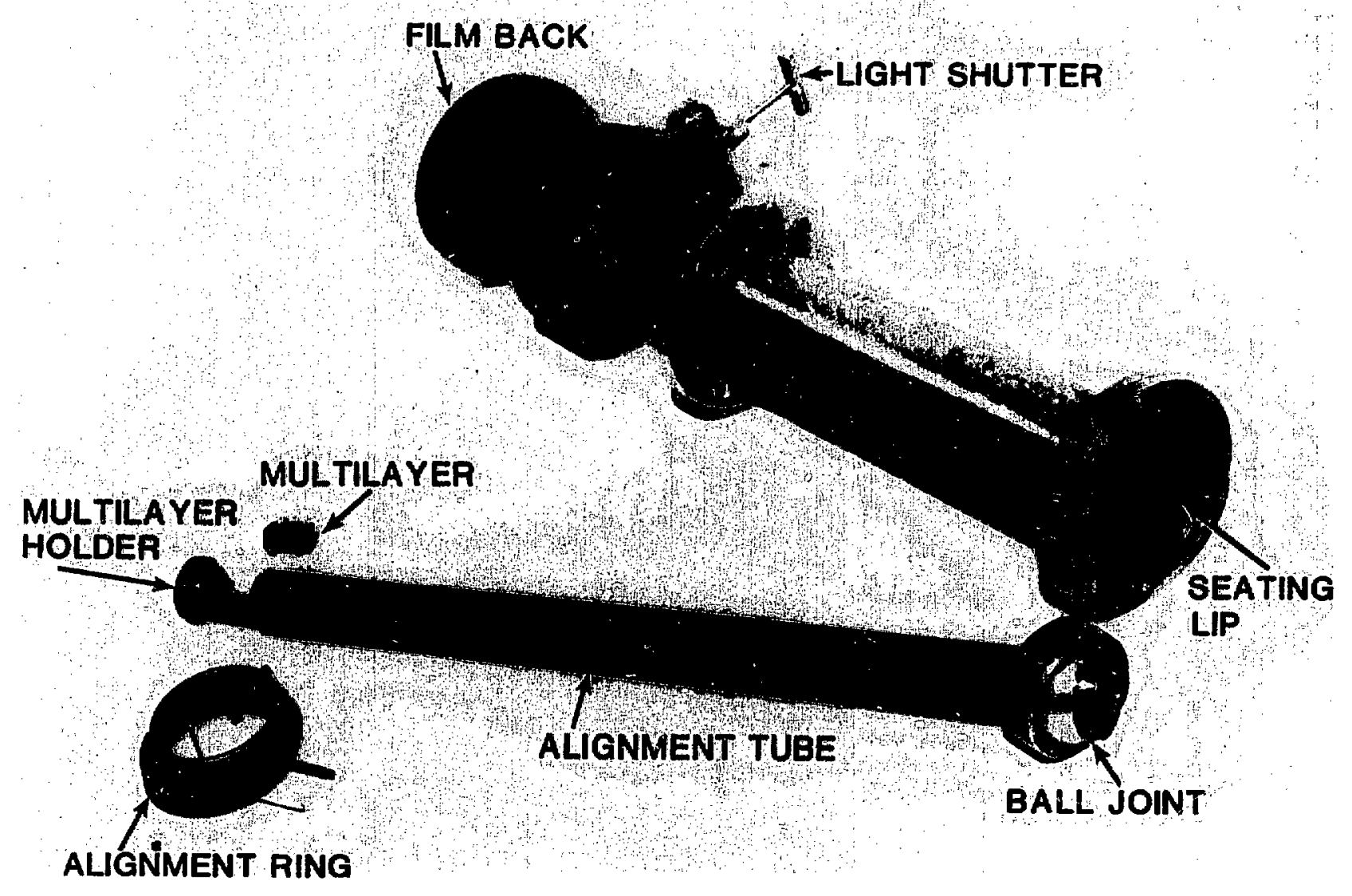


be directed onto the source position. The laser was removed after alignment had been completed.

\section{Experimental Run}

The experiments took place from 15 October 1984 to 17 December 1984. During that period the machine averaged four shots a week. Not all shots used neon gas. Some had xenon and some had krypton as the implosion gas. In addition, different targets of polyacrylic acid foams and parylene "soda straws" on which the gas puffs were imploded were used. These targets provided a variety of sources with different spectral properties and different total energy outputs.

During the first four shots the experiment was fielded. Spectrometer 1 at 13.5 degrees was found to be so long as to be an obstruction and to unbalance the top of the vacuum enclosure. The tube on which the spectrometer was mounted was therefore shortened. The distance between the source and the multilayer was then $150 \mathrm{~cm}$.

In addition, Spectrometer I was experiencing debris problems. Hot gas as well as molten return-current wire was being blasted stralght up the spectrometer pipe onto the control multilayer, destroying the prefilter on the way. A 75-degree port that had been previously assigned 
to another experiment became available, and Epectrometer 1 was shifted to that port. The 75-degree port and the 13.5-degree port were differsnt distances from the source, making it then necessary to lengthen the tube the spectrometer was mounted on. The debris problem was decreased further by placing a circular 2-cm inner-diameter baffle in the spectometer tube at $86.4 \mathrm{~cm}$ from the source.

The change in port assignments also made it more likely that both spectrometers were looking at a similar cross section of the source. This reduced intensity variations due to different source-viewing angles. Spectrometer 1 was posjtioned 45 degrese azimuthally from Spectrometer 2. A revised schematic is shown in Figure 24.

Because the source characteristics exhibited shotto-shot variation of intensity and continuum content, there were probiems getting "clean" spectra of the neon resonance lines. Different prefilter and postfilter material combinations of aluminum, beryllium, parylene, aluminum oxide, parylene, Kimfol, and Kapton were tried to optimize the spectra. The best filter combination was determined to be one layer of $2 \mu_{m}$-thick Kinfol plus 0.3 mils of aluminum foil as the prefilter and 4-5 layers of 2-Hm Kimfol and $0.5-m i l s-t h i c k$ aluminum foil as the post 


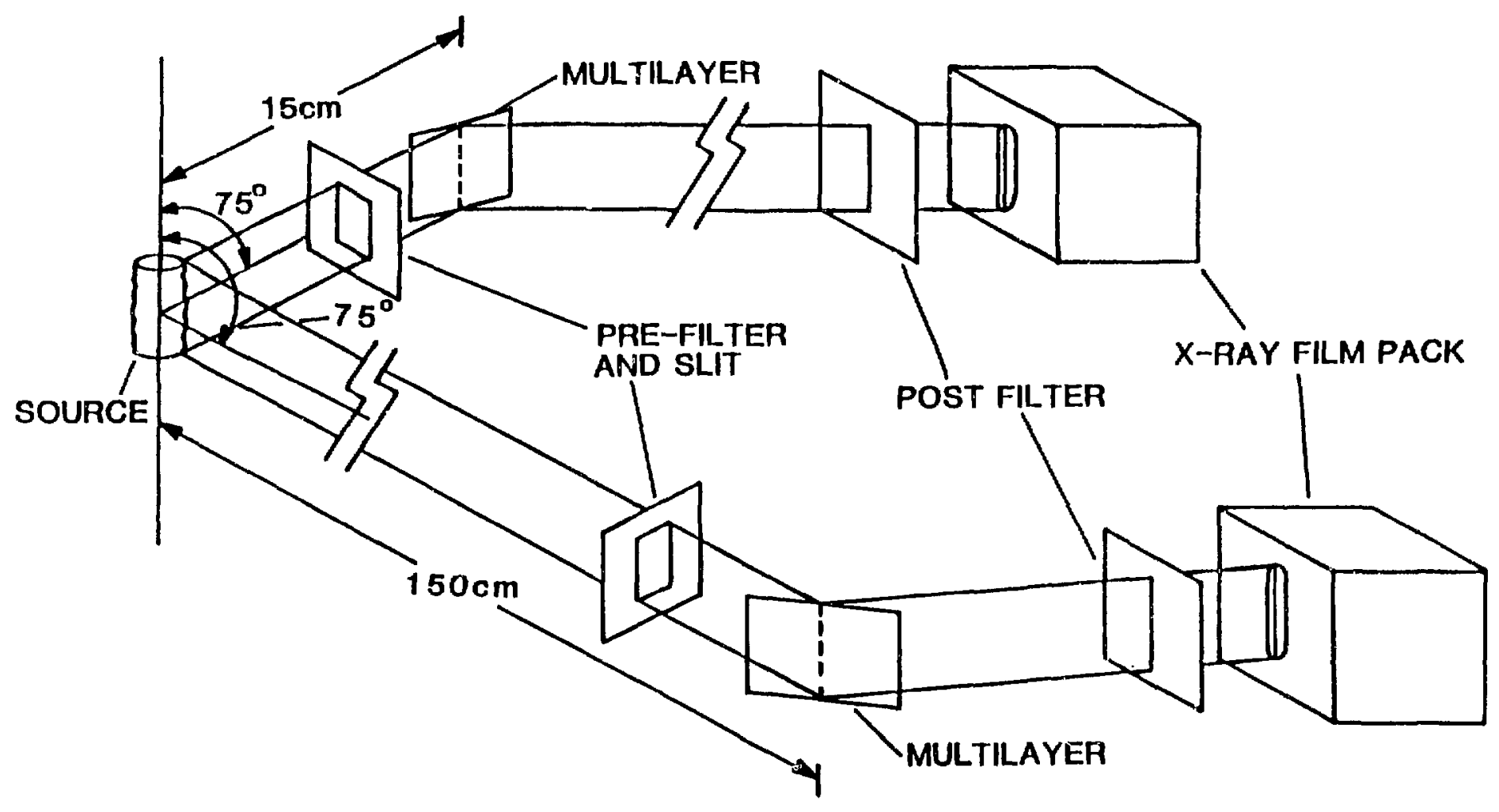

Figure 24. Revised schematic of the two multilayer epectrometerg used at PROTO II. 
filter for neon target shots. The transmission characteristics of this filter combination are shown in Figure 25 .

Another problem encountered and solved was the determination of the proper $x-r a y$ film to be used. SB-5 and RAR 2497 x-ray films were used side by side and back to back to determine different contributions to background and signal. It was finally decided to use RAR 2497 in front and $5 B-5$ in the back to give spectral ranges of $900 \mathrm{eV}-1.8 \mathrm{keV}$ for the $\operatorname{RAR} 2497 \mathrm{film}$ and $>3$ keV for the sB-5 film. The energy response of the $f$ ilm was taken to be that of Henke et al. ( $1984 a$ and b), and their prescribed processing procedures were strictly followed. Figure 26 gives the effective energy sensitivity of the final film configuration.

After the multilayers were exposed to RRoTo II, they were taken back to the laboratory, and their x-ray reflection properties were remeasured as a function of angle at $C u L_{\alpha}$ wavelength. All the multilayers that had been used in Spectrometer 2 (only $15 \mathrm{~cm}$ from the source) were so scorched and covered with debris that they no longer had any Bragg alffraction properties. The debris was hot gas, molten return-current wires, and stainless steel. Attempts were made to clean the debris off the multilayers but to no avail. Those multilayers that had 


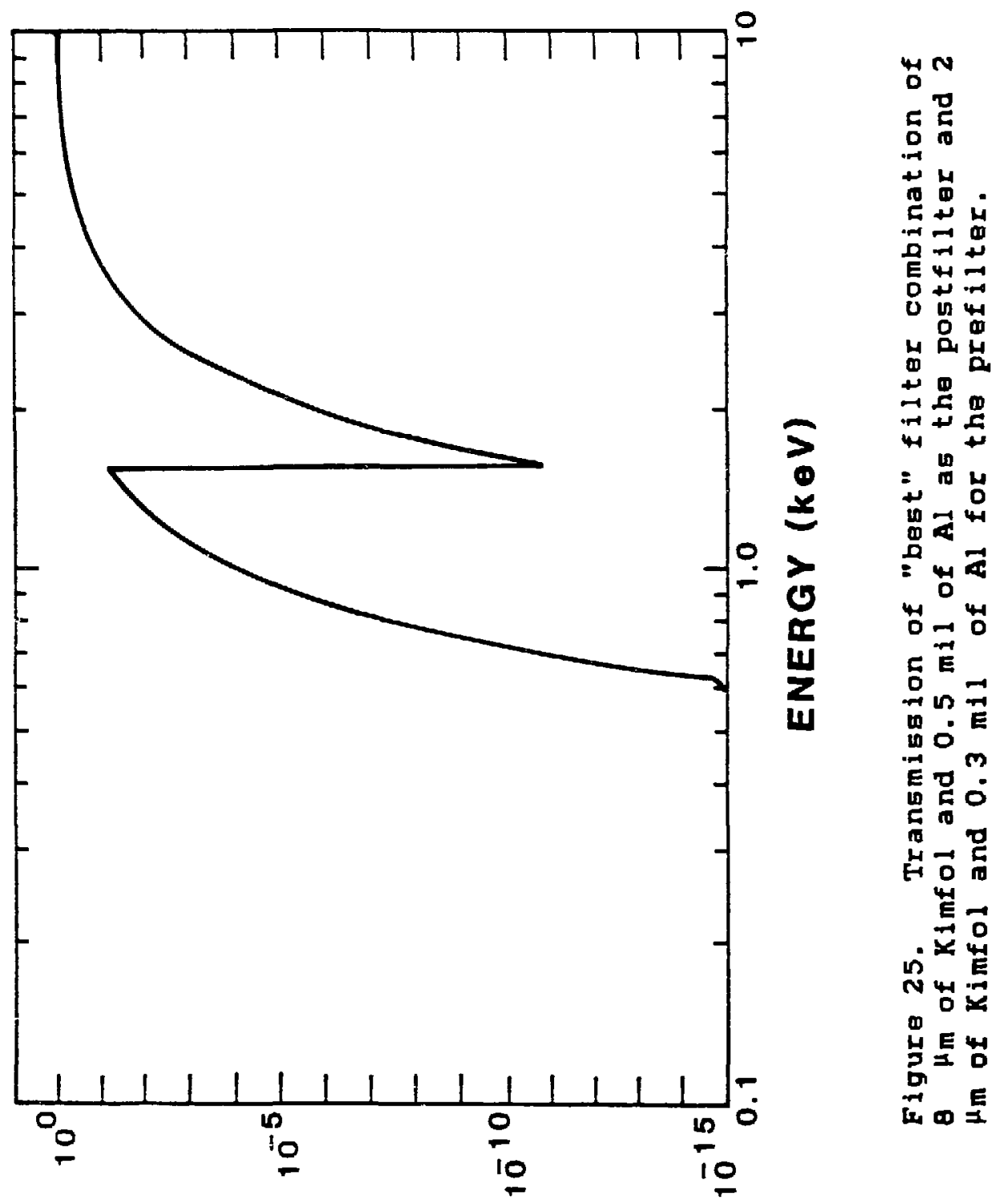

NOISSIWSN $\forall 4 \perp$ 


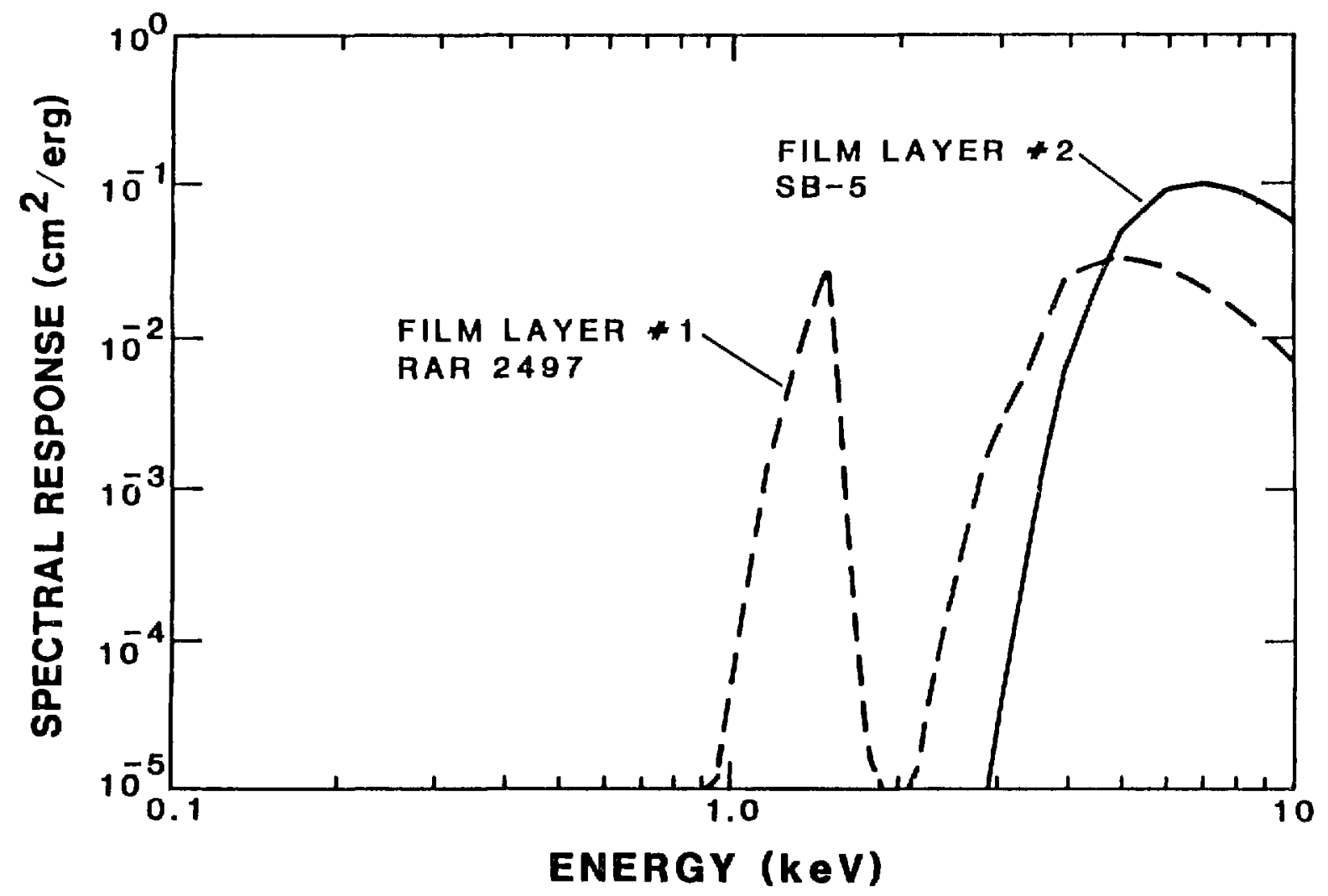

Figure 26. Spectral Eensitivity of final film configuration used in the multilayer spectrometers. 
been used in spectrometer 1, though they had a few marks from hot gas. were remeasured to have the same reflection properties as before. The multilayers used in Spectrometer 1 could therefore be used again.

A summary description of the useful shots for the time-integrated run is found in Table 2 . Shots \#1789, \#1805, and \#1806 used the molydenum-carbon. long dspacing multilayers that required the smallest Bragg angle for diffracting the neon resonance lines. For these shots, each spectrometer detected the presence of two spectral lines. Preliminary identification bassd on the position and spacing of the two lines was made to be the hydrogen- and helium-like resonance lines observed with the KAP curved-crystal spectrograph as shown previously in Figure 11 of Chapter II-1. The spectral lines of Spectrometer 2 were very large and widely spaced compared with thos of Spectrometer 1. This was due to the difference in the separation between the multilayer and the detector in the two spectrometers. In addition, the large width of the spectral lines on both apectrometers was due to the finite extent of the source. The diameter of the source was 2-5 $\mathrm{mm}$ depending on the shot, and not the expected $2 \mathrm{~mm}$. The diameter of the source was determined from the pinhole photographs.

Shot 1798 was the only shot for which good data 
TABLE 2. SUMMARY OF USEFUL TIME-INTEGRATED NEON GAS SHOTS.

\begin{tabular}{|c|c|c|c|c|c|c|}
\hline \multirow[b]{2}{*}{$\begin{array}{l}\text { Shot } \\
\text { Number }\end{array}$} & \multirow[b]{2}{*}{ Target } & \multicolumn{2}{|c|}{ Bolometer } & \multicolumn{2}{|c|}{ Multilayer } & \multirow[b]{2}{*}{$\begin{array}{l}20 \\
0 \\
\langle A\rangle\end{array}$} \\
\hline & & $\begin{array}{l}\text { Line } \\
\text { Yield } \\
(k J)\end{array}$ & $\begin{array}{l}\text { Total } \\
Y \text { ield } \\
(k J)\end{array}$ & $\begin{array}{c}\text { Spectrometer } \\
1 \\
(\text { far })\end{array}$ & $\begin{array}{c}\text { Spectrometer } \\
2 \\
\text { (near) }\end{array}$ & \\
\hline 1796 & $\mathrm{CH}$ straw & 10.3 & 21 & $83-021-I I$ & .5 of $83-021-I$ & 72 \\
\hline 1798 & $\begin{array}{l}\text { Ti-coated } \\
\text { straw }\end{array}$ & 12.2 & 44 & $83-023 a-I V$ & .5 of $83-023 \mathrm{~A}-\mathrm{V}$ & 116 \\
\hline 1805 & foam & B. 8 & 30 & $83-023 \mathrm{~A}-\mathrm{II}$ & .5 of $83-023 A-I$ & 116 \\
\hline 1806 & foam & 7.0 & 18 & $83-023 A-I I$ & 5 of $83-023 \mathrm{~A}-$ III & 116 \\
\hline
\end{tabular}


were obtained using a $70-8$ multilayer, and that was only on Spectrometer 1. This was because the shorter $\underline{d}-$ spacing multilayers required the larger angles. Setting the multilayer of spectrometer 2 at these larger angles proved to be difficult, and the line of sight to the source could have been blocked by the return current posts.

Conclusions that covld be arawn from the $t$ imeintegrated data were that the spectral lines could indeed be recorded for the larger 2d-spacing multilayers and that the basic design of the spectrometers was sound. "In addition, these data gave needed rough intensity numbers and physical parameters with which to design a $t$ imeresolved detector system. 
CHAPTER II-3

TIME-RESOLVED EXPERIMENTS

The Experimental Design

The purpose of this phase of the investigation was to observe changes in $x-r a y$ reflectivity properties of metal muliflayers as a function of time and irradiance. If the multilayer were heated by the incident flux, a possible angle shift in the reflected line profile due to thermal expansion of the layers, as well as a possible widening of the diffraction pattern due to decreasing order among the layers, might be expected.

As previously mentioned, the nominal duration of the neon resonance $x-r a y$ lines was $20 \mathrm{~ns}$. If 10 time frames or bins were necessary to observe the expected changes, a system with approximately 2-ns time resolution was needed. The spatial requirements for detecting changes in the angular position or widths of the $x-r a y$ diffraction pattern, that is, the spectral lines, could be determined from the time-integrated data. Since the width of a line at the Specirometer 2 detection plane was observed to be $2.5 \mathrm{~mm}$. for a minimum of 10 resolution elements $250-\mu_{m}$-wide resolution elements were needed. These temporal and spatial resolution requirements could be met with a fluorescer-fiber optic-visible streak 
camera system.

Tc determine whether a fluorescer-fiber opticstreak camera system was a viable detector system, it was necessary to calculate whether there inind be a sufficient light signal. A rough calculation was done as follows.

The proposed configuration and parameters necessary to calculate the flux through the detector system are shown in Figure 27. The source temporal FWHM was taken to be $20 \mathrm{~ns}$ and to have $5 \mathrm{~kJ}$ of total energy in each 1 ine at $1022 \mathrm{eV}$ and $922 \mathrm{eV}$. This took into account the increased energy yields seen in the time-integrated run. The intensity available, $I_{p}$ is given by the equation $I_{p} \quad=\frac{E_{1 \text { ine }}}{4 \pi \Delta E_{\text {photon }}}$,

where $E_{\text {line }}$ is the total energy in the line. At is the $x-$ ray pulse width, and $E_{\text {photon }}$ is the photon energy of the line. Therefore,

$$
\begin{aligned}
& I_{p / 1022}=\frac{5 \mathrm{~kJ} \times 1000 \mathrm{~J} / \mathrm{kJ}}{4 \pi \mathrm{EI} \times 1022 \mathrm{eV} / \mathrm{ph} \times 1.6 \times 10^{-19} \mathrm{~J} / \mathrm{eV} \times 20 \times 10^{-9} \mathrm{~s}} \\
& =1.216 \times 10^{26} \mathrm{ph} / \mathrm{sr}-\mathrm{s}, \text { and } \\
& I_{P / 922}=\frac{5 \mathrm{~kJ} \times 1000 \mathrm{~J} / \mathrm{kJ}}{4 \pi 5 \mathrm{E} \times 922 \mathrm{eV} / \mathrm{ph} \times 1.6310^{-19} \mathrm{~J} / \mathrm{eV} \times 20 \times 10^{-9} \mathrm{~s}} \\
& =1.349 \times 10^{26} \mathrm{ph} / \mathrm{sr}-\mathrm{s} \text {. }
\end{aligned}
$$

The flux at the fluorescer was calculated by the 


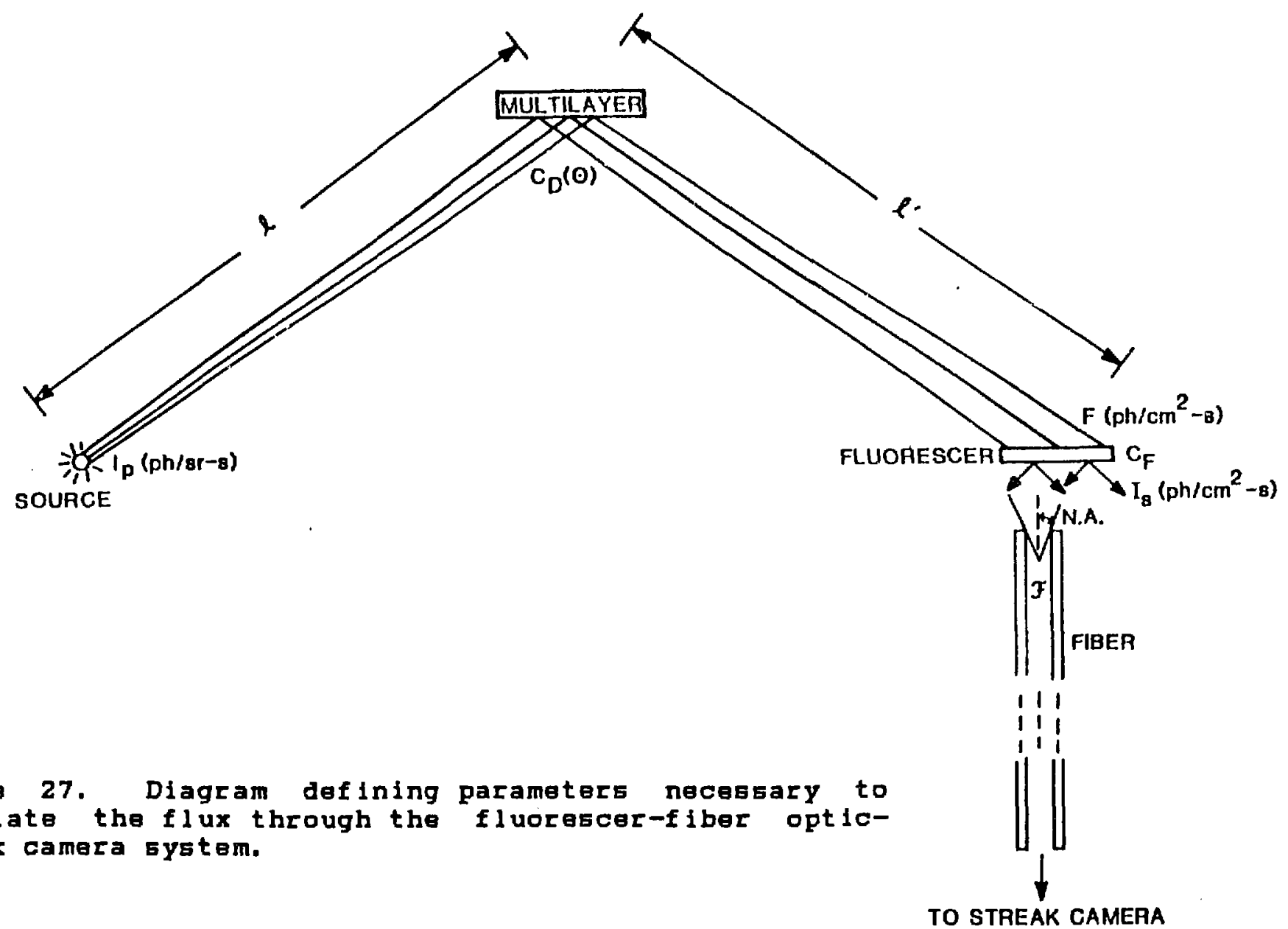

Figure 27. Diagram defining parameters necessary to calculate the flux through the fluorescer-fiber opticotreak camera system.

TO STREAK CAMERA 
equation

$F=\frac{I_{p} d \varphi d \theta \theta_{d}\left(\theta_{B}\right) \cos (\alpha)}{d X d H}$

where dy is the angular "height" of the multilayer intercepted at th: aptection plane, de is the angular "length" of the multilayer intercepted at the detection plane. $C_{d}\left(\theta_{B}\right)$ is the peak reflectivity of the crystal function, $\underline{d x}$ is the resolution width of the line at the detection plane, and $\underline{d H}$ is the resolution height at the detection plane. The resolution width, dx, was taken to be the physical width of the lines observed by Spectrometer 2 during the time-integrated experiments, that is, $3 \mathrm{~mm}$. The resolution height, $\underline{\mathrm{dH}}$, was taken to be the height of the film exposed in Spectrometer 2 during the timeintegrated run, that s, $1.905 \mathrm{~cm}$. The angles dr and de were calculated according to the diagram in Figure 28 to be 0.0227 rad and $3.58 \times 10^{-3}$ rad, respectively. Nominal reflectivity numbers for the peak of the crystal function, $C_{d}\left(\theta_{B}\right)$, were extrapolated from laboratory measurement to be

$C_{\mathrm{d}}\left(\theta_{\mathrm{B}}\right)=0.04$ at $1022 \mathrm{eV}$ and $C_{\mathrm{d}}\left(\theta_{\mathrm{B}}\right)=0.03$ at $922 \mathrm{eV}$.

Therefore, the flux at the fluorescer for the two lines was eslculated to be

$F_{1022}=\frac{1.216 \times 10^{26} \mathrm{ph} / 5 \mathrm{r}-5 \times\left(0.0227 \times 3.57 \times 10^{-3}\right) \mathrm{Er} \times .04}{0.3 \mathrm{~cm} \times 1.905 \mathrm{~cm}}$ 

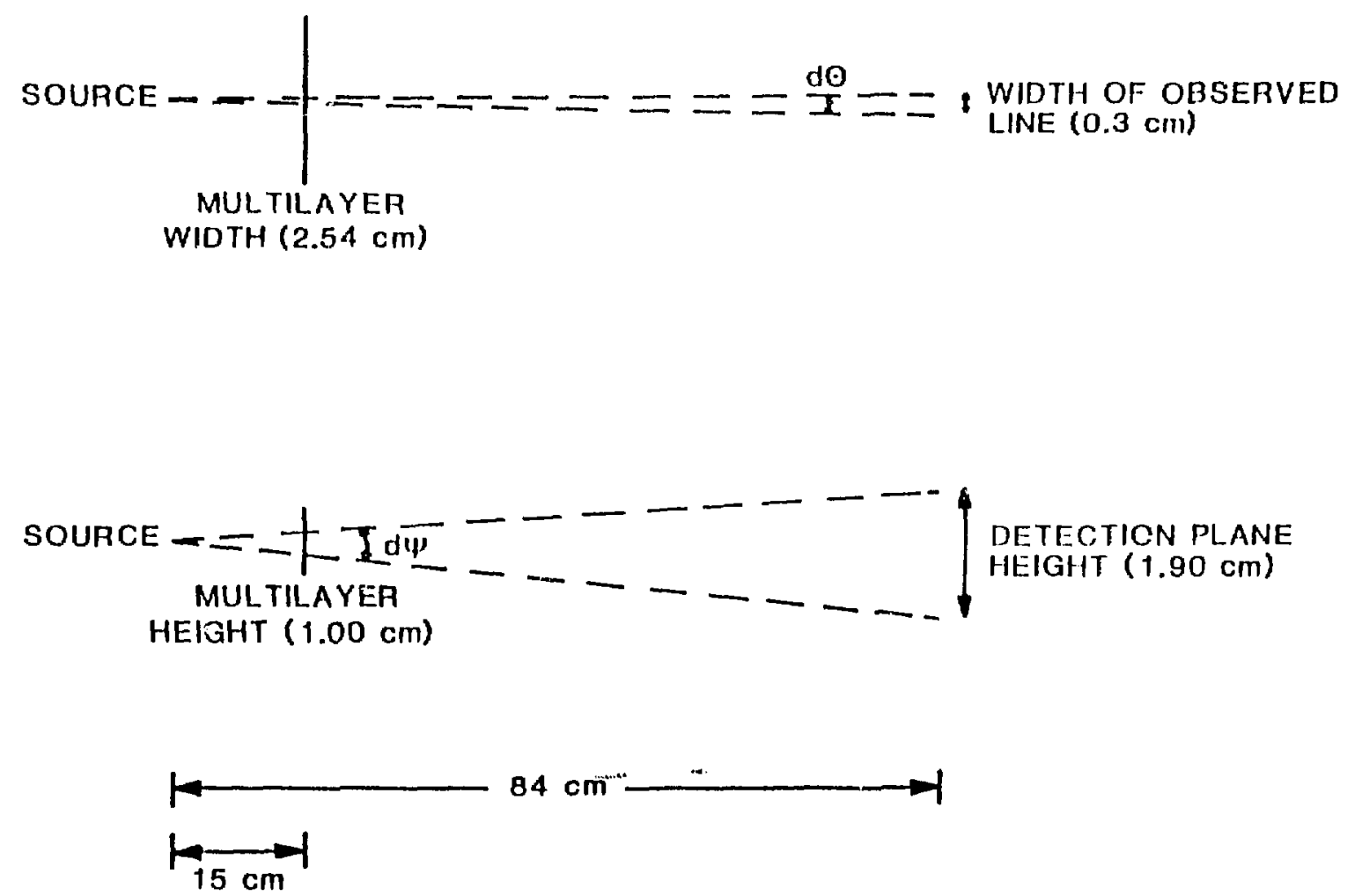

Figure 28. Derivation of dy and de. 


$$
\begin{aligned}
& =6.897 \times 10^{20} \mathrm{ph} / \mathrm{cm}^{2}-\mathrm{s}, \text { and } \\
\mathrm{F}_{922} & =\frac{1.349 \times 10^{26} \mathrm{ph} / \mathrm{sr}-\mathrm{s} \times\left(0.0227 \times 3.57 \times 10^{-3}\right) \mathrm{sr} \times .04}{0.3 \mathrm{sm} \times 1.905 \mathrm{~cm}}, \\
& =5.739 \times 10^{20} \mathrm{ph} / \mathrm{cm}^{2}-\mathrm{s} .
\end{aligned}
$$

The light intensity at the other side of the fluorescer is given by the equation

$I_{S}=C_{F} F$

where $C_{E}$ is the conversion efficiency of the fluorescer. The conversion efficiency of a 2-mil-thick fluorescer was approximated (Lyons and Lier, 1975) for one keV photons to be $4.974 \times 10^{-4}$. Therefore for the two energies

$$
\begin{aligned}
I_{s / 1022} & =4.974 \times 10^{-4} \mathrm{sr}^{-1} \times 6.897 \times 10^{20} \mathrm{ph} / \mathrm{cm}^{2}-\mathrm{s}, \\
& =3.43 \times 10^{17} \mathrm{ph} / \mathrm{cm}^{2}-5 e c-5 r, \text { and } \\
I_{s / 922} & =4.974 \times 10^{-4} \mathrm{sr}^{-1} \times 5.739 \times 10^{20} \mathrm{ph} / \mathrm{cm}^{2}-5, \\
& =2.85 \times 10^{17} \mathrm{ph} / \mathrm{cm}^{2}-\text { sec-sr. }
\end{aligned}
$$

The flux, $F_{t}$ that is within the acceptance angle of the $f$ iber is given by

$$
F_{t}=\sim I_{s} \times(\text { N.A. })^{2} \times \pi \text {. }
$$

where N.A. the numerical aperture of the fiber, was taken to be 0.26 . Therefore,

$$
\begin{aligned}
& F_{t / 1022}=3.43 \times 10^{17} \times 0.2124=7.285 \times 10^{16} \mathrm{ph} / \mathrm{cm}^{2}-5-5 I, \text { and } \\
& F_{t / 922}=2.85 \times 10^{17} \times 0.2124=6.063 \times 10^{16} \mathrm{ph} / \mathrm{cm}^{2}-5-5 I .
\end{aligned}
$$

A rough number for the attenuation in a fiber is $\sim 44 \mathrm{~dB} / \mathrm{km}$; that is, for a 50-m fiber there would be a loss of $2.2 \mathrm{~dB}$, which is equivalent to 60.2 percent 
transmission. Therefore, the flux at the other end of the fiber, $F_{5 c^{\prime}}$ would bs $F_{\text {sc/ } / 1022}=0.602 \times 7.285 \times 10^{16}=4.38 \times 10^{16} \mathrm{ph} / \mathrm{cm}^{2}-\mathrm{s}$, and $F_{E C / 922}=0.602 \times 6.063 \times 10^{16}=3.65 \times 10^{16} \mathrm{ph} / \mathrm{cm}^{2}-\mathrm{s}$.

A "rule of thumb" number is that at least $10^{15} \mathrm{ph}$ $\mathrm{cm}^{-2}-\mathrm{s}^{-1}$ are required to exceed the threshold sensitivity of the streak camera. This calculation indicates that there would be a signal about 40 times this threshold; however, there were no corrections for filters or reflection losses at interfaces. According to the results of this calculation, then, a fluorescer-fiber-streak system would work if careful attention were paid to possible signal $1058 \mathrm{~s}$.

\section{The Fiber Bundle}

The available optical fiber was the Corning DBF Short Distance fiber. Its advertised characteristics were attenuation of $4 \mathrm{db} / \mathrm{km}$ at $850 \mathrm{~nm}$ and $2 \mathrm{ab} / \mathrm{km}$ at $1300 \mathrm{~nm}$ with a bandwidth of $200 \mathrm{MHz} / \mathrm{km}$ at both wavelengths. The fiber had an $85-\mu_{m}-d i a m e t e r$ core and an outer diameter of $125 \mu_{m}$. In order to get the signal from the fluorescer to the electromagnetic impulse (EMI) protected streak camera, the length of the bundle had to be $47 \mathrm{~m}$. Attenuation losses in the fiber begin to become significant at this length. EG\&G, Las Vegas Operations (LVO) measured 
the attenuation of the DBF Fiber used in making the $f$ iber bundile as a function of wavelength, and their results are shown in Figure 29. Their Optical Multichannel Analyzer System could not go below $419 \mathrm{~nm}$; thus, extrapolating to the wavelength of the peak of emission of NE111 gives approximately $66 \mathrm{~dB} / \mathrm{km}$ or a loss of 30 percent for a 47m-long ífber. This is 8 percent more than had been used in the threshold calculation. In addition EG\&G. LVO measured the fiber'g numerical aperture for two different lengths of $f$ iber to be 0.24 and 0.25 .

The time-integrated data were used to determine the physical configuration of the fiber bundle. The major constraint to the number of $f$ ibers that could be used to cover the two spectrometers' detection planes was the size of the streak camera's photocathode. A large photocathode was needed. An RCA-tube-based camera with a 3cm-diameter photocathode was available, and the fiber bundle was designed with this in mind.

The two spectral lines detected with Spectrometer 2 during the $t$ ime-integrated experiments were approximately $3 \mathrm{~mm}$ wide, and separated by 6-7 $\mathrm{mm}$. By comparing all available data, it was determined that a bundle that had two sets of 56 fibers separated by $6 \mathrm{~mm}$ would be sufficient to cover these two lines if the position of the fiber bundle could be adjustable for different d- 


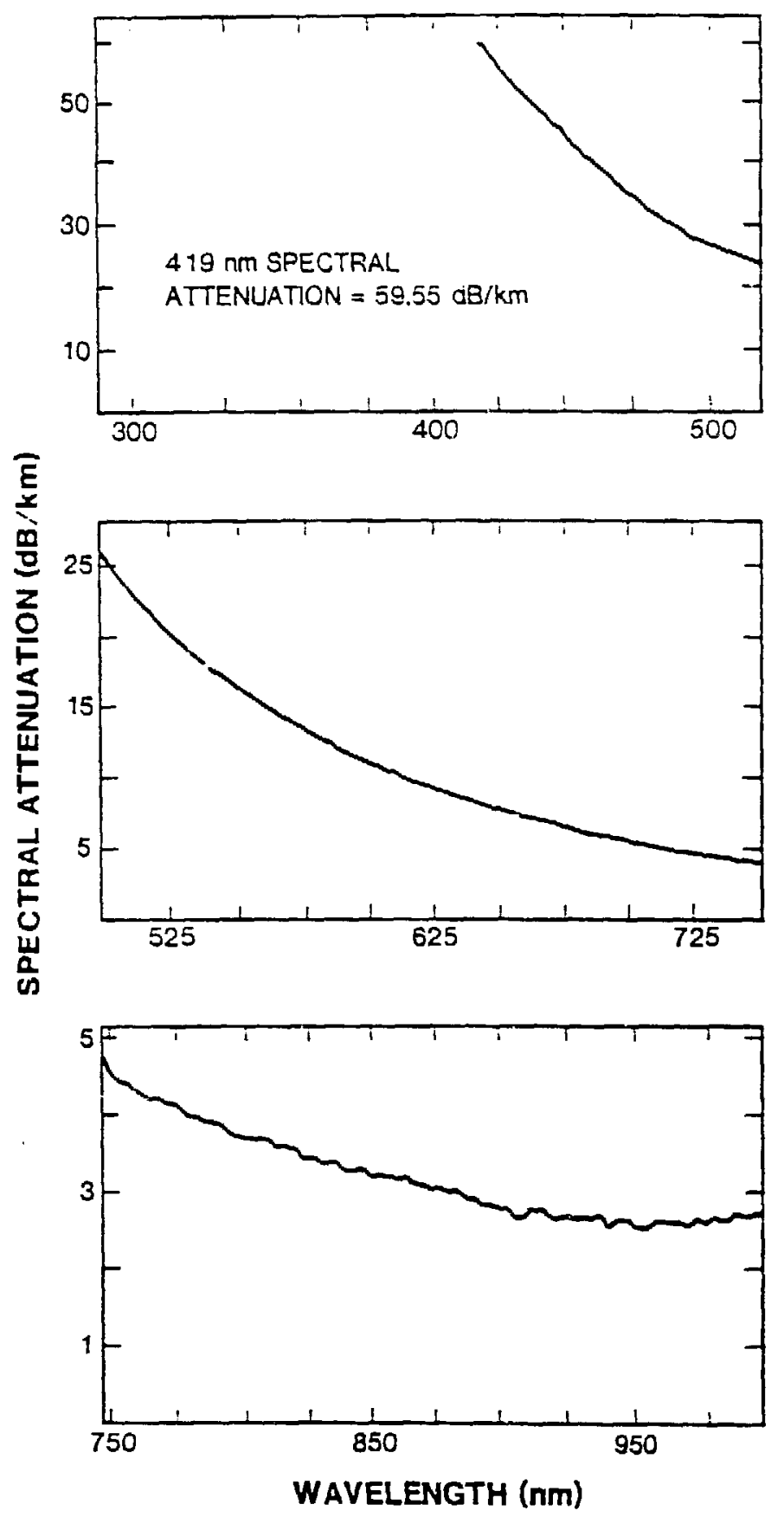

Figure 29. Spectral attenuation as a function of wavelength of the DBF $f$ iber for three different wavelength ranges. 
spacing multilayers. The linear distance at the streak camera end would then be $2 \times 56 \times 125 \mu_{\mathrm{m}}$ or $14 \mathrm{~mm}$.

The 1 ines on Spectrometer 1 were much closer together. By analyzing the time-integrated data, it was concluded that 64 fibsrs were enough to cover the spectral lines if the bundle's position could be adjusted for different d-spacing multilayers. The linear distance at the streak camera for this array would therefore be the same as at the fluorescer end, that is, $8 \mathrm{~mm}$. Figure 30 shows the configuration of the fiber bundle.

In order to position the fiber bunde arrays at each spectrometer, two holders were designed on optical linear positioning stages. The holder-stage of Spectrometer I was positioned directly with a micrometer. The holder-stage of Spectrometer 2 could not be positioned directly because of the close tolerances the jpectr? ieter tube had with the pulse lines of PROTO II. A right angle gear drive was therefore designed to position the holderstage of Epectrometer 2. Photographs of the holder-stages are shown in Figures 31 and 32 for spectrometer 1 and Spectrometer 2, respectively. An important design consideration was the necessary capability of maneuvering the fiber bundle face flat up against the vacuum faceplate window for most efficient coupling. 


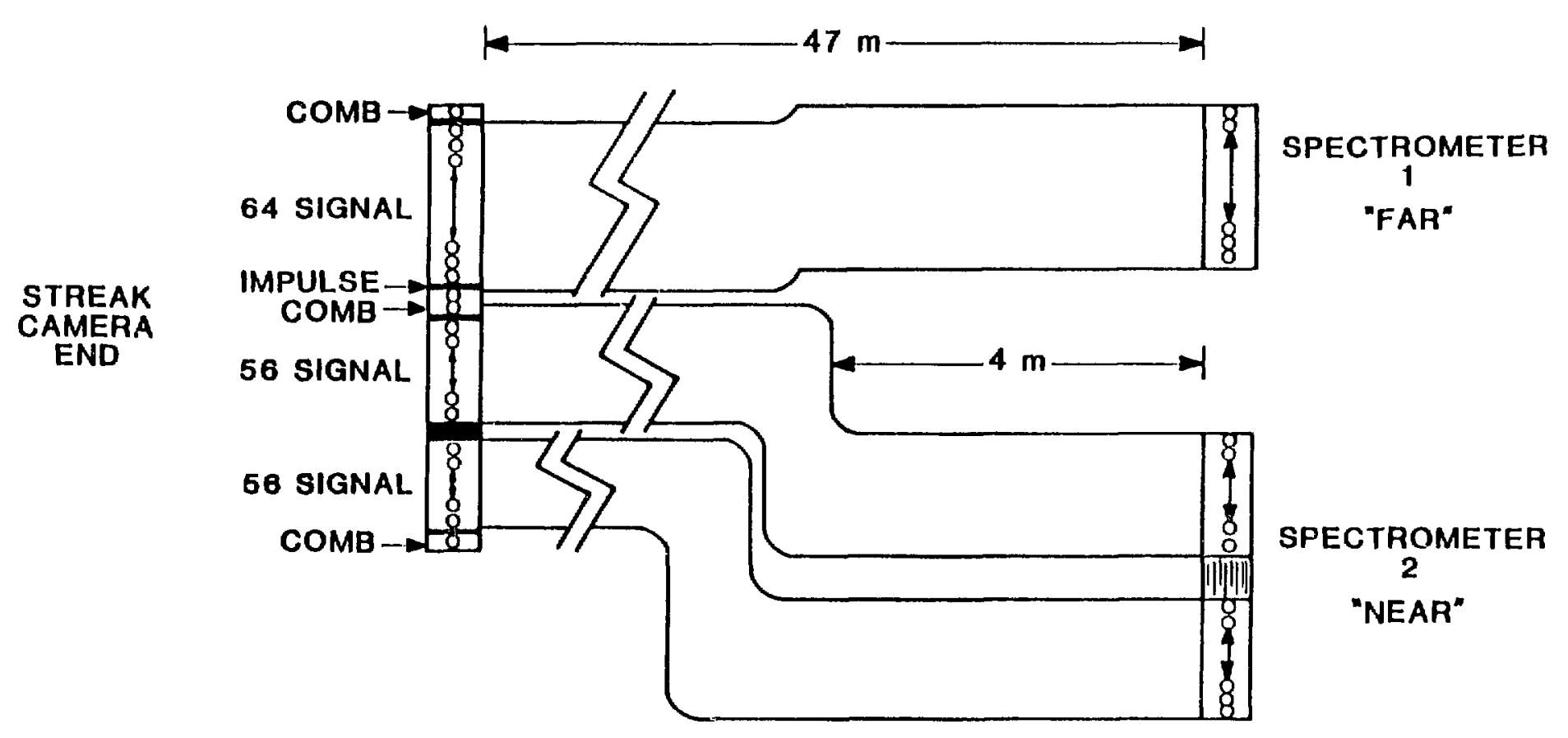

Figure 30. Conflguration of the fiber bundle ends. 
Figure 31. Photograph of the fiber bundle holder-stage assembly for spectrometer 1 . 


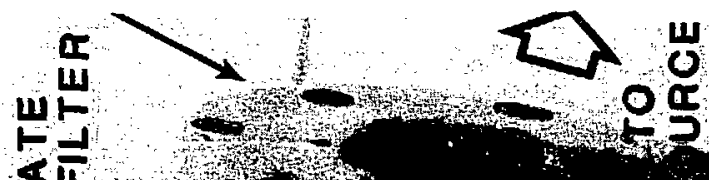

$46 x$
जo
080

$1 \infty \mathbf{I}$

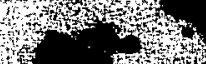


Figure 32. Photograph of the fiber bundle holder-stage asssembly for Spectrometer 2 . 


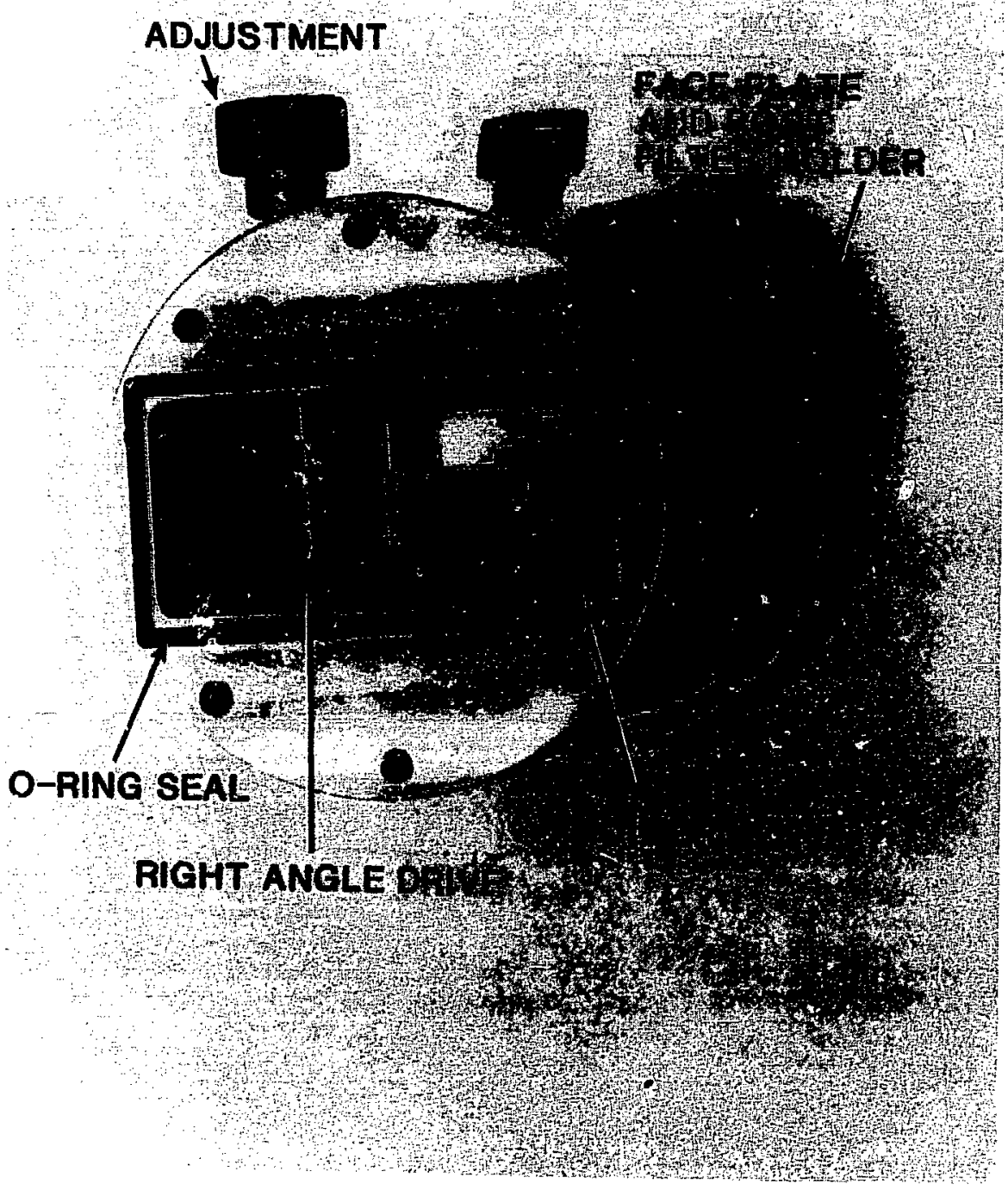


The usable part of the 3-cm streak camera photocathode was the center $\sim 2.7 \mathrm{~cm}$. The length of the $f$ iber arrays needed to cover the two spectrometers' detection planes was $2.2 \mathrm{~cm}$, leaving $0.5 \mathrm{~cm}$ that sould be used for calibration fibers.

\section{The Fluorescer}

There were three main considerations for determining which fluorescer to use. The first consideration was that the fluorescer was to be used in a vacuum; therefore, a solid fluorescer was deemed most practical within the imposed time constraints. The second consideration was the need to satisfy the time-resolution requirement. The final consideration was the availabilty of the fluorescer chosen.

The two most commonly used fluorescers for photons in the keV energy range are Nel11C and NE102C. Their spectral output has been studied extensively (Pronko et al.. 1978 and 1979). Their intrinsic temporal widths have been measured (EG\&G, 1981) to be $1.9 \mathrm{ng}$ for NElll and $2.9 \mathrm{~ns}$ for NE102. It is important to note that the total integrated spectrai output of NElll is approximately twice that of NEIO2.

In addition to the intrinstc temporal FWHM of the fluorescer itself, the energy dispersion properties of the fiber to be used had to be considered. Typical 
fiber optic dispersion numbers (Ogle, 1985) at the two energy peaks of the respective fluorescers are 50.5 ps/nm-100ft and $34 \mathrm{ps} / \mathrm{nm}-100 \mathrm{ft}$ of fiber for NElll and NE102 light, respectively. Taking the geometric mean of the intrinsic FWHM and the dispersion in a 50-m fiber for each fluorescer gives

${ }_{\text {FWHM }}{ }_{\mathrm{NE} 111}=\left((1.9 \mathrm{~ns})^{2}+(3.9 \mathrm{~ns})^{2}\right)^{1 / 2}=4.3 .3 \mathrm{~ns}$ and FWHM $_{\text {NE1O2 }}=\left((2.9 \mathrm{~ns})^{2}+(1.6 \mathrm{~ns})^{2}\right)^{1 / 2}=3.31 \mathrm{~ns}$.

In the above formula the largest component of the FWHM for NElll is the dispersion term, while for the NE102 it is the intrinsic FWHM. Therefore, considerable gains in the time resalution could be made if the dispersion term of the NElll could be decreased by using an interference filter. A BARR ${ }^{C}$ interference filter was available that had been specifically designed to peak near the NElll wavelength and to 1 imit the FWHM of its radiation to $20 \mathrm{~nm}$. Dr. Donald Casperson of Los Alamos National Laboratory had run a spectrophotometer scan on this filter, and this is shown in Figure 33. The integrated transmission of the interference filter is 0.21 over the NElll emission band. The subsequent dispersion contribution using the interference filter was then only $1.56 \mathrm{~ns}$. This gave for the resultant effective temporal FWHM,

FWHM $=\left((1.9 \mathrm{~ns})^{2}+(1.56 \mathrm{~ns})^{2}\right)^{\frac{1}{2}}=2.45 \mathrm{~ns}$. 


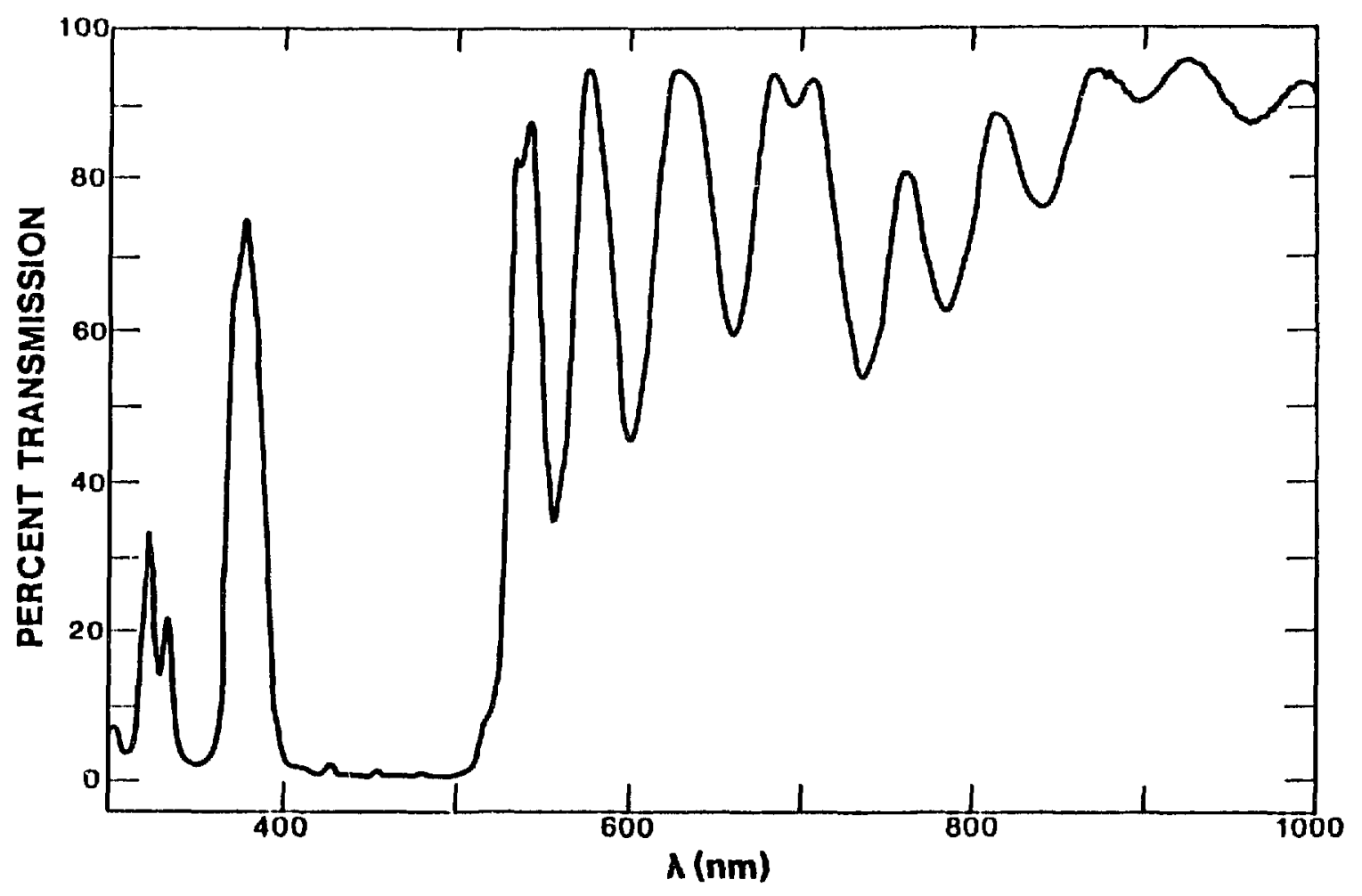

Figure 33. Spectrophotometer scan of BARR interference filter showing percent transmission as a function of wavelength. 
The combination of the BARR filter and the NElll fluorescer gave the nearest values to the previously stated temporal resolution reguirements using available materials.

The problem of getting the light that is converted by the fluorescer out of the vacuum and into the fiber bundle had to be taken into consideration. This was done by using a fiber optic faceplate as the vacuum window and "butt" coupling the fluorescer to the faceplate. The faceplate obtained was reported to have a transmission of 258 (Galileo Electro-Optics Corp., 1981), with $5.8-\mu_{m}$ outer diameter fibers, a numerical aperture of 1.0 , and a thickness of $4.1 \mathrm{~mm}$.

The fiber bundle was designed to cover each spectral line of Spectrometer 2 with 8 fibers. The addition of the fluorescer, the interference filter, and the faceplate necessitated re-examining the spatial resolution of the system. The thicker the fluorescer and interference filter, the larger the width of a signal a fiber would see. Convenientiy, pieces of reported $50-\mu_{m}-$ thick NElli were available. The Barr interference filter was also reported to have a thickness of $50 \mu \mathrm{m}$.

The configuration proposed and the contributing factors to the spatial resolution are depicted in Figure 34. Even though the numerical aperture of the fiber 


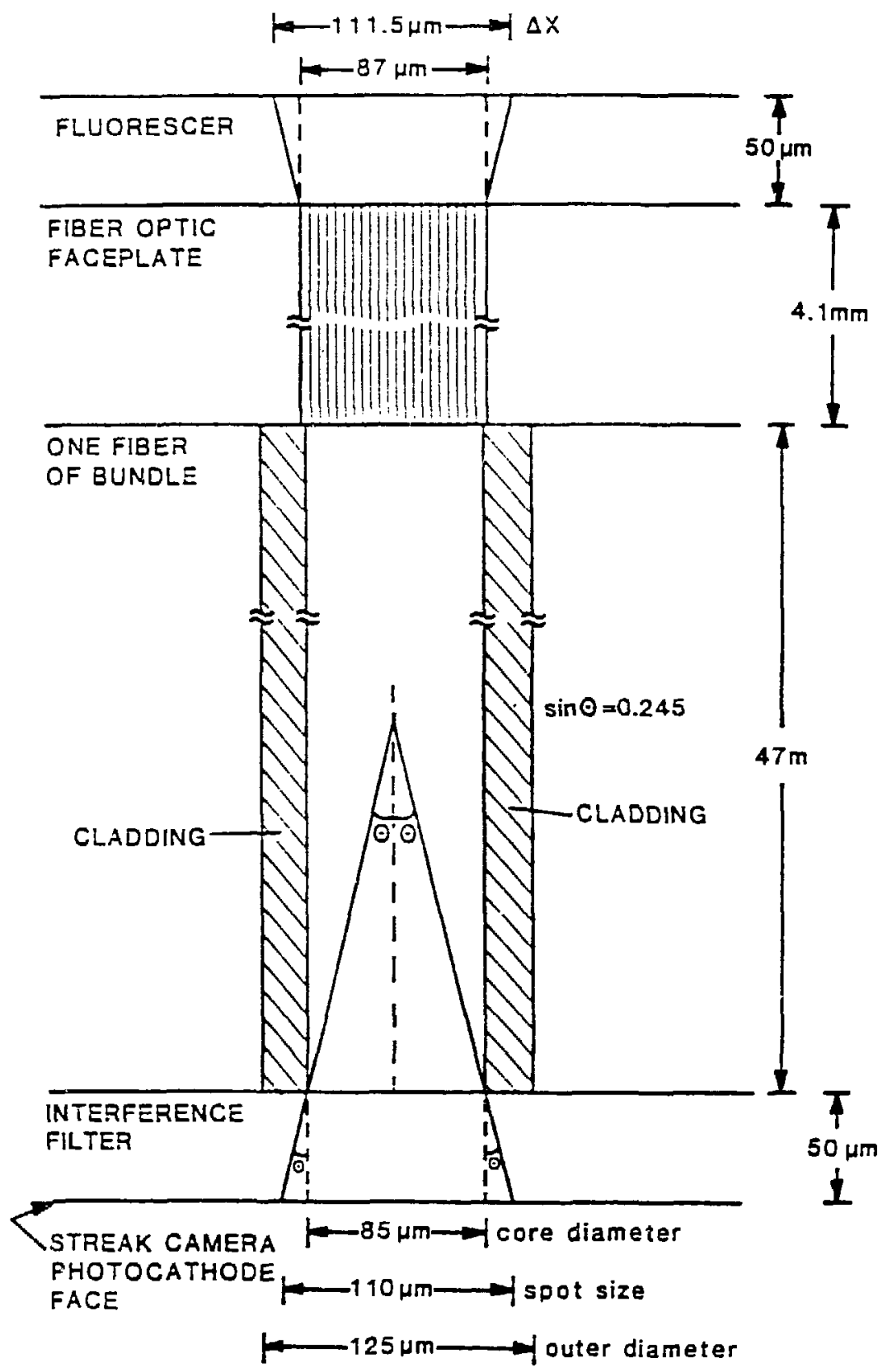

Figure 34. The effects of the fluorescer and interference filter on the spatial resolution, $\Delta x$, of the system. The spot eize on the photocathode is less than the outer diameter of the fiber: therefore, the spatial resolution is determined at the fluoracer and of the system. 
faceplate is 1.0, the effective numerical aperture of the systen is determined by the component with the smallest numerical aperture. In this case it is the numerical aperture of the $f$ ibers in the fiber bundle, 0.245 . Since the fiber faceplate is made up of $5.8-\mu_{m}$ fibers, it takes 14.66 faceplate fibers to cover the diameter of a single fiber of the bundle. The amount of light that is collected by one fiber in the bundle is actually determined by the next nearest integer value, or 15 fibers in the faceplate. Since those 15 fibers have an effective numerical aperture of 0.245 , then, at the top of the fluorescer the spot size will be $111.5-4 m$ diameter. This 111.5 - im diameter is smaller than the outer diameter of the $f$ iber in the fiber bundle. On the other end, the bundle is "butt" coupled directly to the interforence filter and the interference filter is coupled to the streak camera photocathode. The spread at the photocathode surface using the numerical aperture of 0.245 is therefore $110.3 \mu \mathrm{m}$. This is also less than the outer diameter of the flbers in the bundle as well as the spread at the top of the fluorescer. For this reason, the spatial resolution of the system is determined by that at the fluorescer, or input end.

Because the fluorescer was to be so thin, any highenergy component (second or third order of the multi- 
layer) of the incident spectrum would not be completely absorbed by the fluorescer but would be partly transmitted to the faceplate itself. There was some concern that the faceplate would also fluoresce, contributing to a background signal. However, since the BARR filter was on a glass cover slip, placing it directly between the fluorescer and the faceplate might lower any fluorescent background if the cover slip had a lower efficiency than the faceplate. These concerns led to a series of measurements using $T i K_{\alpha} \times$ rays as a source for determining the difference in fluorescent yields. From these data it was concluded that it was best to put the fluorescer directly in front of the faceplate and to put the interference filter outside the vacuum system to minimize the background signal.

\section{East Valve}

As described in Chapter II-2, the standard multilayer in Spectrometer 1 continually had damage due to debris. Since the debris arrived later than the $x$ rays, a fast valve could be used to protect the multilayer from the debris and still pass the x-ray signal.

A model 130 fast valve from Spectral Precision, Inc., with a $1-c m X$ 2-cm aperture and a l-ms closing time was obtained for this purpose. A watertight aluminum box with o-ring 
seals was used to protect the electronics on the valve itself. In addition, the power cords to the valve were shielded inside a grounding strap and were wrapped several times around ferrite toroids to protect t4e fast valve from the electromagnetic impulse which occurred when PROTO II was fired. The valve had an insertion length of 2 inches and was placed immediately in front of the multilayer holder assembly where the manual valve had been during the time-integrated run.

\section{The Streak Camera System}

The streak camera system, built and supported by EG\&G, Los Alamos Operations (LAO), was an RCA-tubebased-modular streak camera. The photocathode was an S20 type and the phosphor was P-20. A type II microchannel plate image intensifier (MCP) was butt-coupled to the streak camera, and Polaroid Type 57 and Kodak RXP filme were used in the accompanying filmbacks. The resultant dynamic resolution of the streak camera was 10 $1 \mathrm{p} / \mathrm{mm}$. The camera was used mostly at $2.6 \mathrm{~ns} / \mathrm{mm}$. Figure 35 shows a photograph of the streak camera.

The camera was supplied with an optical impulse generator and an optical comb generator. The optical comb generator emitted $4 \mathrm{mw}$ of $812-\mathrm{nm}$ light down an attached optical fiber every 2 ns. This comb signal was 
Figure 35. The EG'̈G streak camera. Shown here are the components of the film back, the microchannel plate (MCP), the streak tube housing, and the fiber optic bund le mount. 


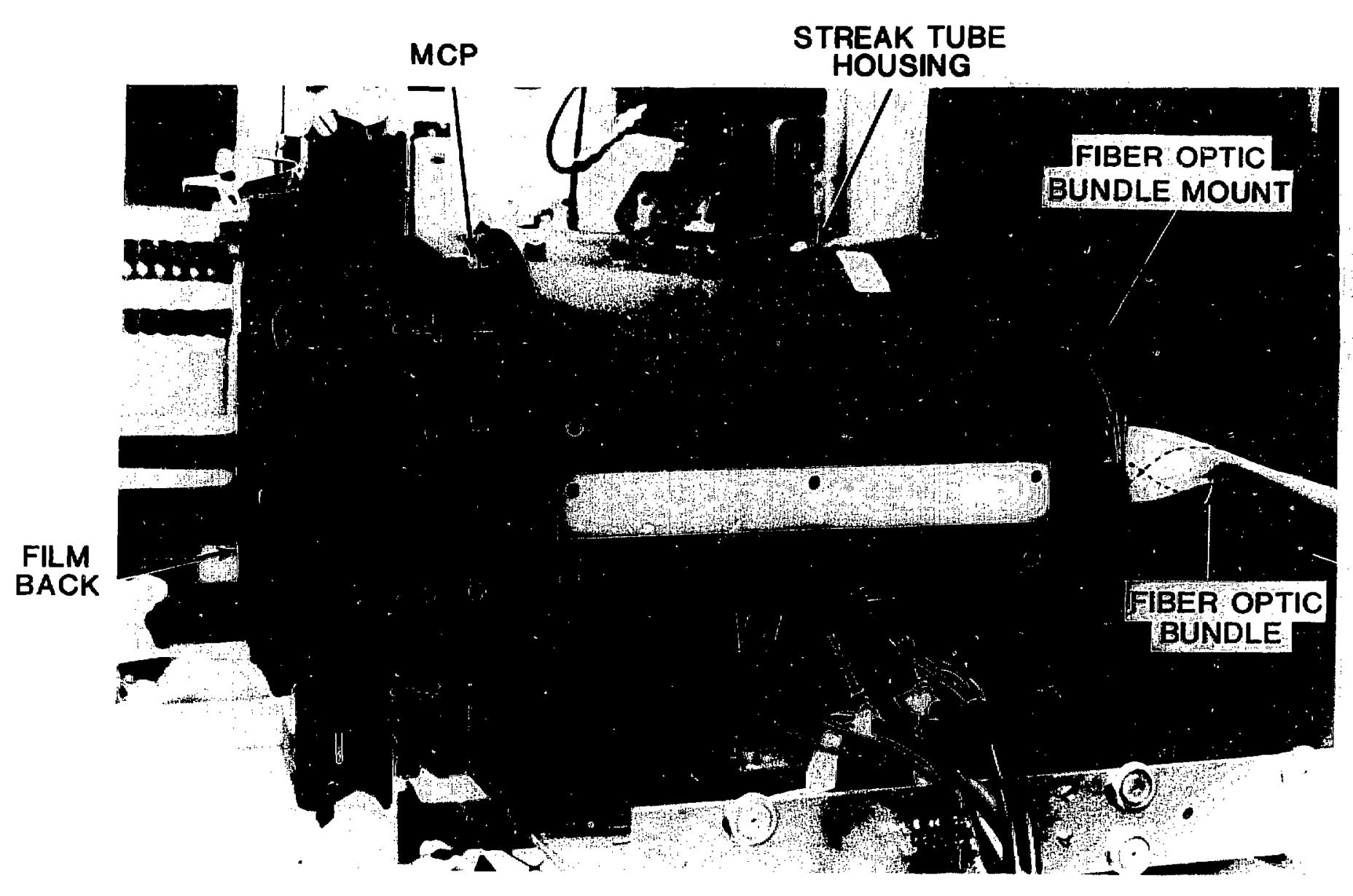


then split into three fibers by a fiber splitter to bracket each set of data with timing marks. The optical impulse generator emitted $15 \mathrm{~mW}$ of $823-\mathrm{nm} 1$ ight down an attached fiber at the prescribed set $t$ ime.

Timing

Determining the correct timing sequence took some care since the optical path of the signal was the same as that of the time calibration signals. The major factors that had to be accounted for in the timing sequence were the delay times associated with the streak camera deflection and turn on, the MCP's delay, and the delay in the comb and impulse gererators. Other factors included the jitter in the line switches and the variable implosion times. A schematic of the timing for the streak camera is shown in Figure 36. The timing points of interest at the PROTO II machine end are shown in Figure 37.

\section{Overal1 Setup}

The main difference between the time-integrated experimental system and the time-resolved setup is the replacement of the filmbacks with the fiber-fluorescerstreak camera detector system described above. The multilayer holders and alignment procedures for the Spectrometer 2 multilayer were all the same. 


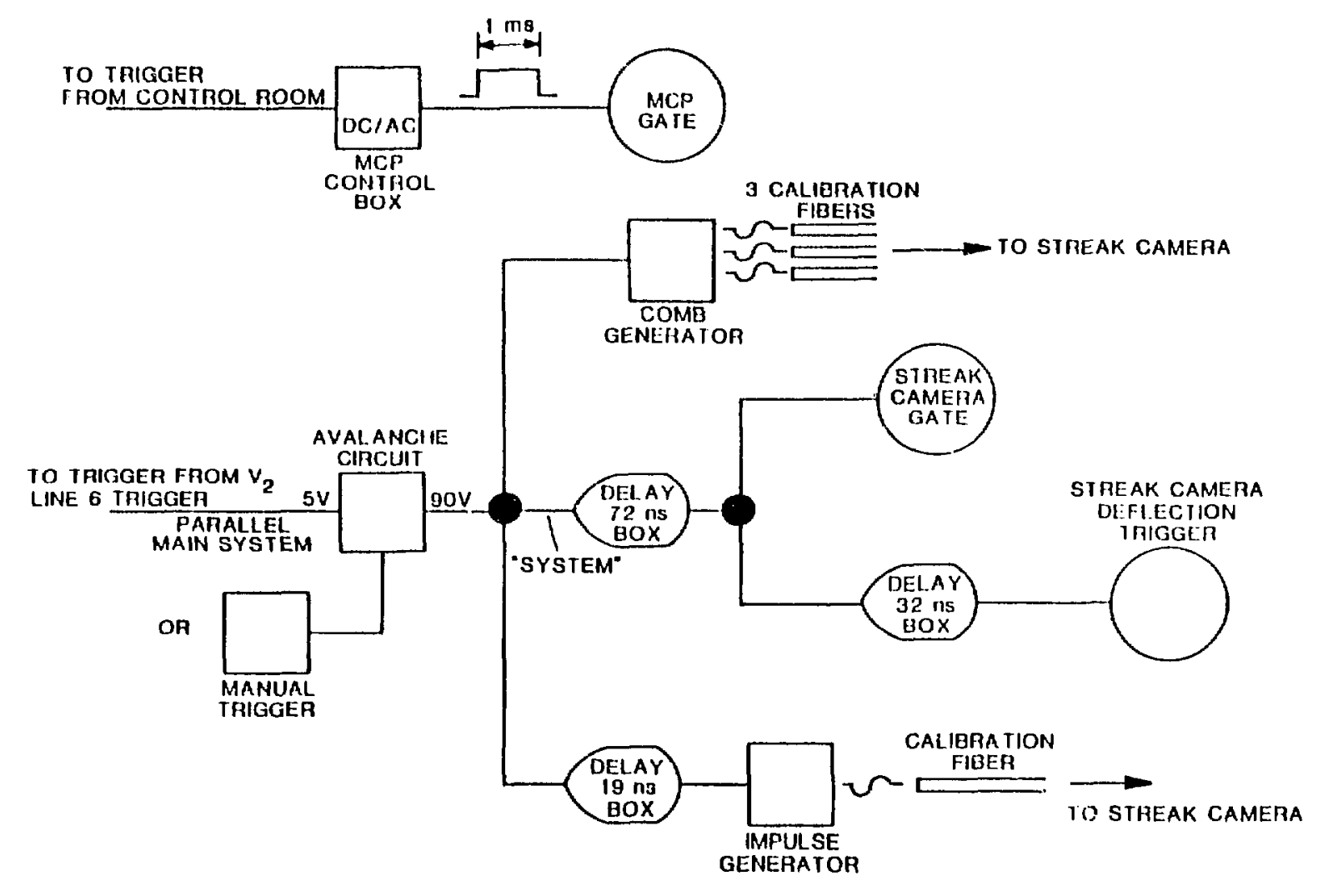

Figure 36. Schematic of the streak camera timing Eequence. 


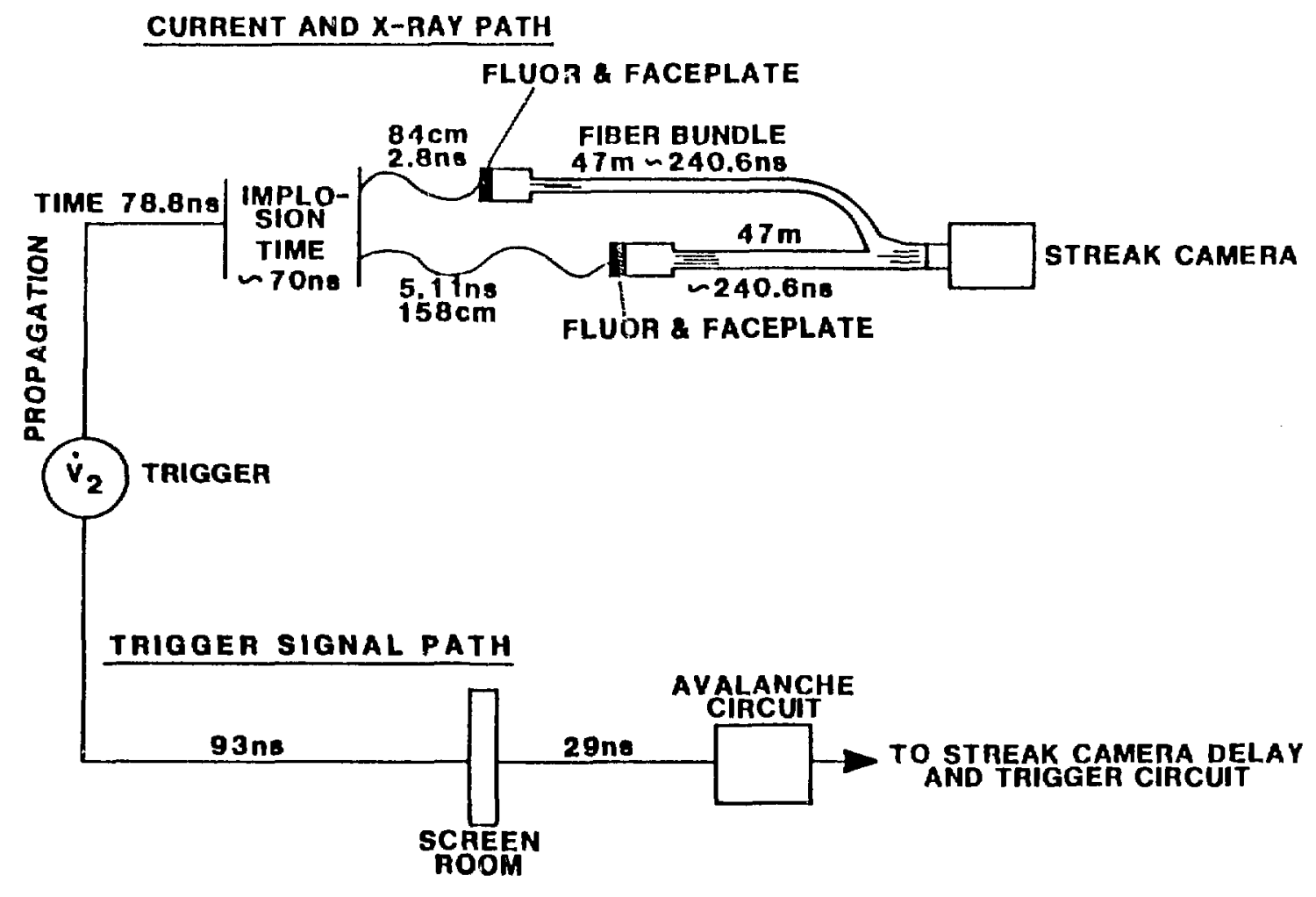

Figure 37. $x$-ray, signal, and trigger paths of the two multilayer spectrometers. 
One change was necessary in order to minimize the length of fiber necessary to reach the screen room and to decrease the possibility of entanglement with people and equipment. Both spectrometers were shifted 1350 to the east of their previous placement, to the other side of the vacuum chamber closer to the screen room.

In order to protect the flber bundle and prevent spurious light from contributing to the signal, the fiber bundle was fed through a curved stainless steel pipe, then a fiexible copper bellows, and then through a conduit all the way to the screen room. Photographs of the two timb-resolved spectrometers as fielded on PROTO II are given in Figures 38 and 39.

\section{Experimental Run}

The time-resolved experiments took place from 21 February 1985 to 18 April 1985. The Proto II experimental team' a emphasis for this run was the implosion of neon gas onto a varlety of targetg. Different targets of metal-doped polyacrylic acid foams and metalcoated parylene "soda straws" were used. At the beginning of the run, it was necessary to use several snots to set the timing of the streak camera and to adjust the filtration. This was complicated by lowyield shots and the lack of any data visible on the ิㅗ $1 \mathrm{~m}$. 
Figure 38. Photograph of the two time-resolved multilayer spectrometers as configured during the run. 


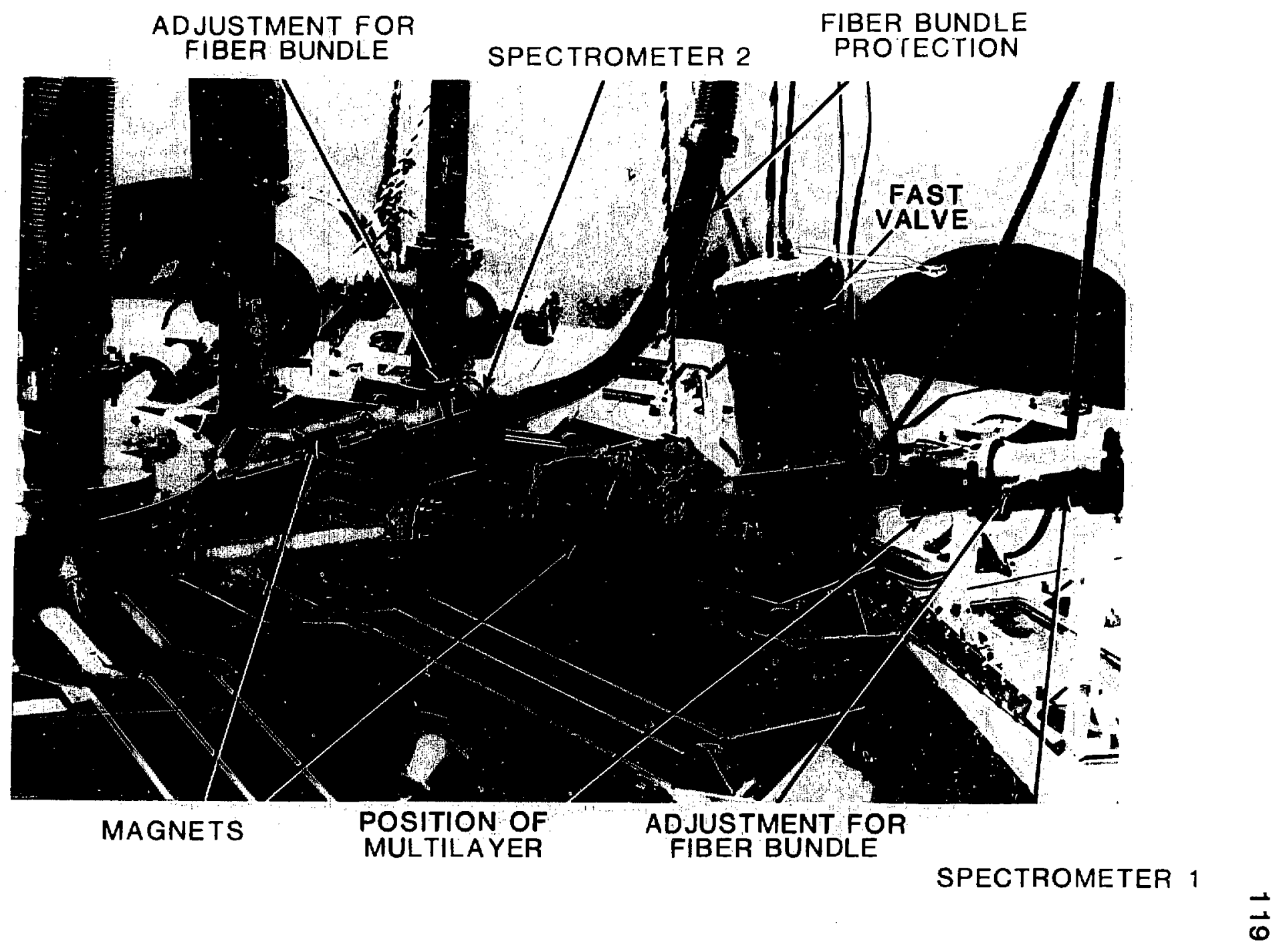


Figure 39. Photograph of the PROTO II vacuum chamber, multilayer spectrometers and the associated radial diagnostics. 


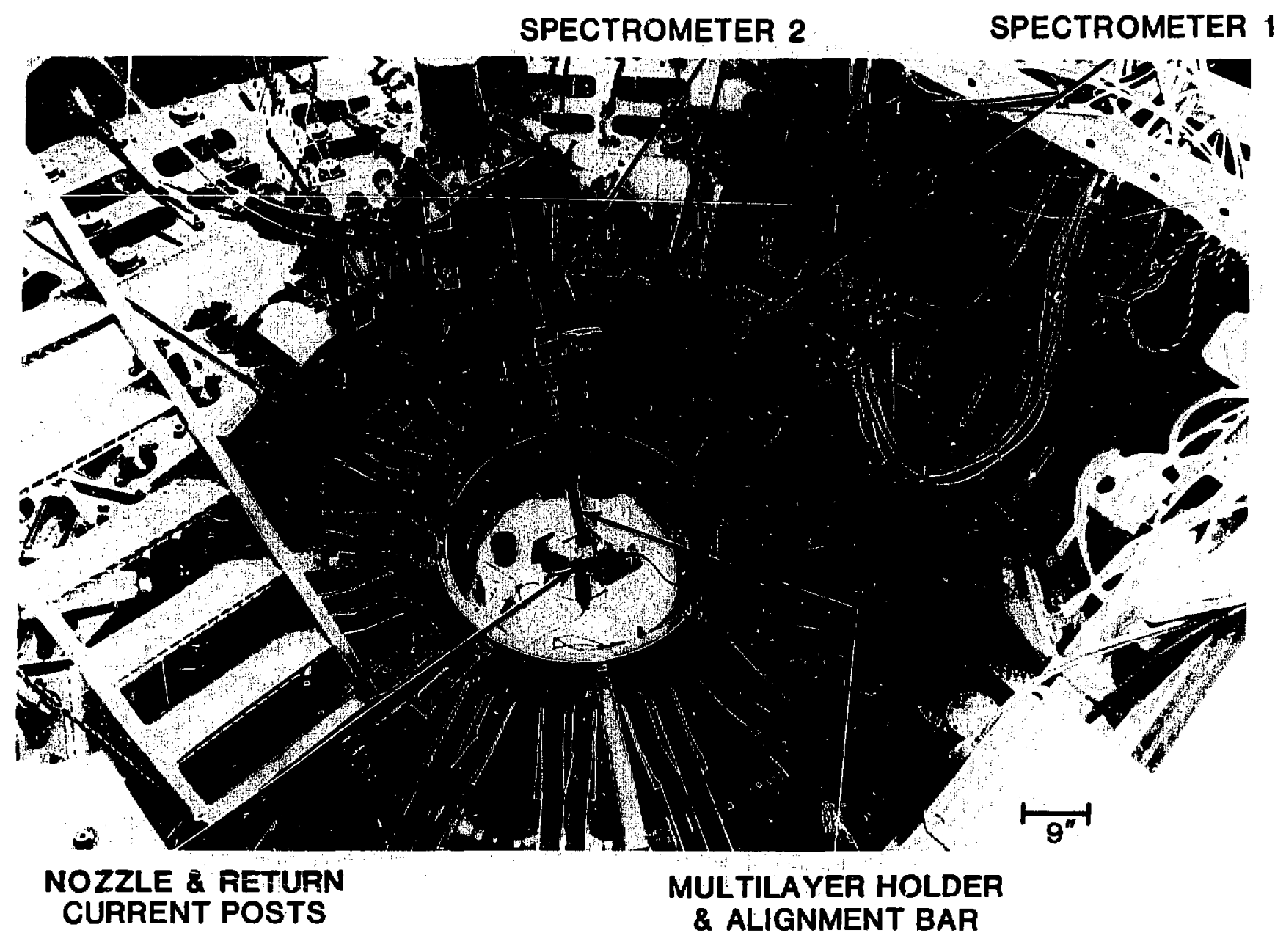


Initial filters for Spectrometer l were a postfilter of a 4- $\mu_{m}$ Kimfol film with a 800-A Al coating and a 2-Hm Kimfol film with a 400-8 Al coating for the prefilter. For Spectrometer 2 the initial filters were a postfilter of $4-\mu \mathrm{m}$ Kimfol film with a $800-8$ Al coating and a prefilter of 5000-A Al foil.

During the pump down of Shot 1857 the postfilter of Spectrometer 2 fell off and no prefilter had been in place: therefore, data obtained were of the white light pulse. This gave a baseline to determine both the iming of the streak camera and the filtration. Because the filters were found to be too thick, they were cut down to an approximate total thickness of $8000 \&$ of $\mathrm{Al}$. The postfilter on Shot *186I had plnholes, causing the streak record to show both the white light and the $x-r a y$ signal. This confirmed that the $x$-ray signal was indeed being detected during the run.

An additional $t i m i n g$ problem that had to be worked out was that of the fast valve. The fllter and/or multilayer was damaged in Spectrometer 1 when the fast valve was not triggered soon enough. The timing was determined at fust about the point in the run where the fast valve electronic box consistently began to take on water. The electronics housing had been constructed out of aluminum and during the course of the shots had 
developed hundreds of tiny cracks in its welds due to the violence of the machine firing. The valve did not operate when the electronics were filled with water. Attempts were made to reweld the box and to seal it with silicon sealant. All the attempts failed. The fast valve was therefore inoperable for the rest of the run, and the debris pjoblem cont inued.

Another problem that was encountered during the run was that when the machine was fired, there was 50 much torque on the threaded joint of spectrometer 1 that it broke the weld. This breakage allowed water into the byetem behind the fiber faceplate, getting the fiber bundle end and holder stage wet. This problem was solved by welding two nuts to the outer threaded piece and bolting it to the other threaded piece. The nuts can be seen in Figure 31.

Depending on the target, the current return posts were of different bizes. Unexpectedly, the thicker halfinch-diameter posts blocked the nose of spectrometer 2. This prevented acquisition of data from Spectrometer 2 for those shots.

During the course of this Iun, positive streak camera data were obtained for 9 out of $19 x$-ray producing shots. 


\section{Data}

A general summary of the time-resolved-data shots and the accompanying Sandia data from the diagnostics that were discussed in Chapter II-l is given in Table 3.

For each shot, the streak camere record on RXP film consisted of a step wedge, timing combs, an impulse, and the data. Figure 40 shows a print of the streak record from Shot \#1863. The signal from the comb generstor consists of the thres columns of white dots. Each dot is separated by cwo nanoseconds. The step wedge is put on each film such that a specific film density can be related to a known energy density.

The step wedge is exposed onto the film with a spectrum that simulates the light emitted from the P-20 phosphor of the streak camera system. Each of the 26 steps is obtained by placing different neutral density filters between the film and the light source. The step wedge used was constructed by $M$. A. Palmer at Sandia, and its optical and energy density values are given in Table 4.

All streak camera data were digitized by EGG (LAO) with a Perkin-Elmer PDS Microdensitometer. For data analysis, the step wedge was digitized as follows. For each step of the wedge, a $512 \times 19$ pixel array was taken using a $42-\mu_{m} \times 42-\mu_{m}$ square aperture with a $10-\nu_{m}$ step over. 
TABLE 3. SUMMARY OF TIME-RESOLVED DATA SHOTS.

\begin{tabular}{|c|c|c|c|c|c|c|c|}
\hline \multirow[b]{2}{*}{$\begin{array}{l}\text { Shot } \\
\text { Number }\end{array}$} & \multirow[b]{2}{*}{ Target } & \multicolumn{2}{|c|}{ Multilayer } & \multirow[b]{2}{*}{ XRD } & \multirow[b]{2}{*}{ Bolometer } & \multirow[b]{2}{*}{$\begin{array}{l}\text { KAP } \\
\text { Crystal }\end{array}$} & \multirow[b]{2}{*}{ Pinhole } \\
\hline & & $\begin{array}{l}\text { Spectrometer } \\
\text { (Close) }\end{array}$ & $\begin{array}{c}1 \text { Spectrometer } 2 \\
(\text { Far })\end{array}$ & & & & \\
\hline 1858 & V-foam & weak & weak & 4 & good & weak & good \\
\hline 1860 & $v$-foam & weak & weak & 6 & good & weak & good \\
\hline 1861 & $V-f o a m$ & pinholes & weak & 5 & good & good & good \\
\hline 1862 & v-foam & weak & weak & 6 & good & good & good \\
\hline 1863 & none & good & good & 2 & good & good & good \\
\hline 1871 & v-foam & weak & - & 6 & good & good & good \\
\hline 1872 & $v$-foam & good & good & 5 & good & good & good \\
\hline 1874 & Cl-foam & weak & weak & 6 & good & good & good \\
\hline 1875 & $\begin{array}{l}\mathrm{Cu} / \mathrm{AI}- \\
\text { foam }\end{array}$ & - & good & 6 & good & - & good \\
\hline
\end{tabular}


Figure 40. Print of streak record of shot 1863 showing data, combs, impulse, step wedge, and the space that separates the two sections of $f$ iber of Spectrometer 2 . 


\section{SPECTROMETER 2 SPECTROMETER 1}

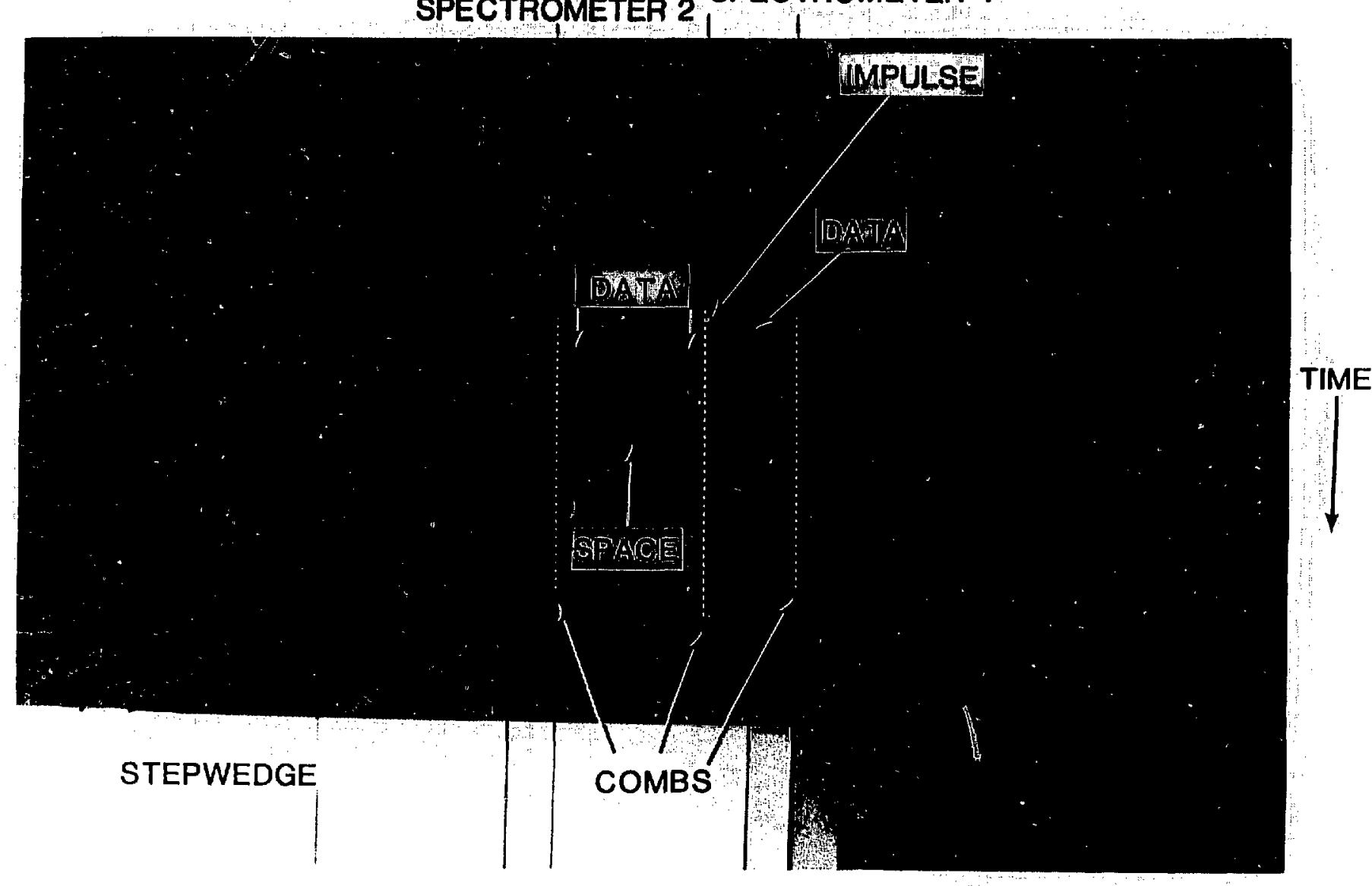


TABLE 4. DENSITIES OF THE STEP WEDGE WITH CORRESPONDING VALUES OF EXPOSURE.

\begin{tabular}{|c|c|c|c|c|c|}
\hline $\begin{array}{l}\text { Step } \\
\text { Number }\end{array}$ & $\begin{array}{l}\text { Step } \\
\text { Density }\end{array}$ & $\begin{array}{l}\log _{10} \operatorname{EXP} \\
\left(\mathrm{erg} / \mathrm{cm}^{2}\right)\end{array}$ & $\begin{array}{l}\text { Step } \\
\text { Number }\end{array}$ & $\begin{array}{l}\text { Step } \\
\text { Density }\end{array}$ & $\begin{array}{l}\log _{10} \operatorname{EXP} \\
\left(\operatorname{erg} / \mathrm{cm}^{2}\right)\end{array}$ \\
\hline 1 & 0.04 & 0.5069 & 14 & 2.10 & -1.5531 \\
\hline 2 & 0.20 & 0.3469 & 15 & 2.26 & -1.7131 \\
\hline 3 & 0.35 & 0.1969 & 16 & 2.43 & -1.8831 \\
\hline 4 & 0.51 & 0.0369 & 17 & 2.60 & -2.0531 \\
\hline 5 & 0.66 & -0.1131 & 18 & 2.77 & -2.2231 \\
\hline 6 & 0.82 & -0.2731 & 19 & 2.93 & -2.3831 \\
\hline 7 & 0.98 & -0.4331 & 20 & 3.10 & -2.5531 \\
\hline 8 & 1.14 & -0.5931 & 21 & 3.24 & -2.6931 \\
\hline 9 & 1.30 & -0.7531 & 22 & 3.40 & -2.8531 \\
\hline 10 & 1.45 & -0.9031 & 23 & 3.55 & -3.0031 \\
\hline 11 & 1.61 & -1.0631 & 24 & 3.72 & -3.1731 \\
\hline 12 & 1.78 & -1.2331 & 25 & 3.87 & -3.3231 \\
\hline 13 & 1.94 & -1.3931 & 26 & 4.02 & -3.4731 \\
\hline
\end{tabular}


The optical density is defined as the negative base $10 \log$ of the transmission observed through the film. The optical densities determined from the step wedge are then used to produce a "lookup" table of density versus exposure.

For the data, the entire streak camera record was digitized with an array of $512 \times 512$ pixels, using a 42$\mu_{m} \times 42-\mu_{m}-\varepsilon q u a r e$ aperture with a $50-\mu_{m}$ stepover. The array was then "straightened" by computer with respect to the combs. By the use of a Comtal system, an average over any number of columns (distance) or rows ( $t$ ime) could be taken and plotted. The time scale is determined by the number of pixels between the comb dots. This averaging process is known as "compressing the streak." The compressed streak is what will be compared with the calculations.

Figure 41 shows such an average for Shot \#1863 for corresponding signals of the same feature for each spectrometer. The data have been smoothed using the adaptive filtering code AWARE (Hodson et al., 1981). AHARE takes a weighted average of the data points according to the steepness of time data. The signal from Spectrometer 1 is larger than that of Spectrometer 2 , in part because Spectrometer 1 exhibits higher background exposure due to its more straight-through line of sight. 


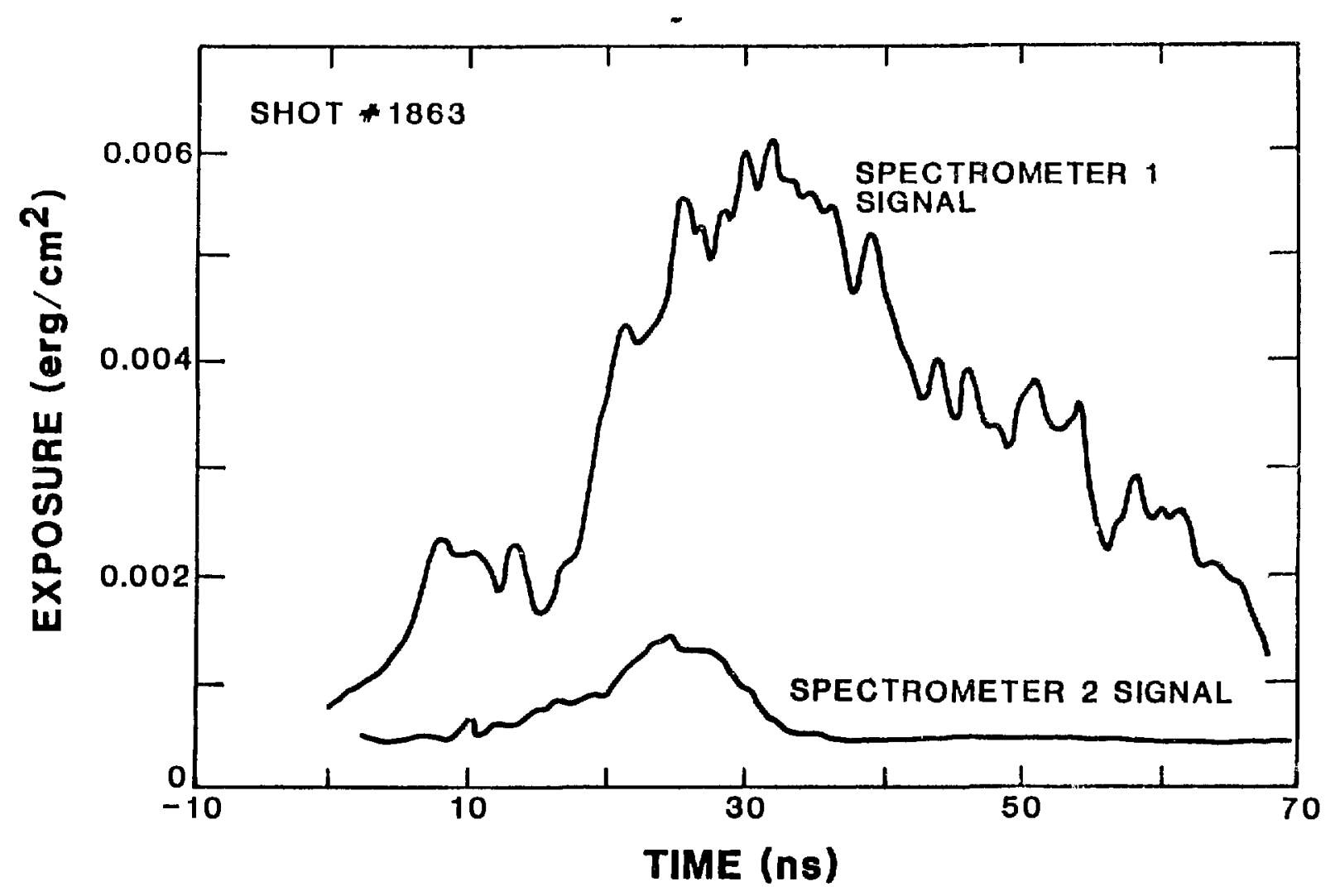

Figure 41. Compressed streak from Spectrometers 1 and 2 for Shot 1863 . Data were smoothed in exposure space using AWARE adaptive filtering code. 
In addition, the compressed streak of Spectrometer 2 is over more pixels than Spectrometer l causing the average exposure to be less. The time history of the signal from Spectrometer I has a FWHM of $\sim 25 \mathrm{~ns}$, which is in agreement with the bolometer signal. The signal from Spectrometer 2 exhibits a FWHM of only $\sim B$ ns and falls off very rapidly, indicating that the multilayer reflecting surface was destroyed before the peak of the radiation pulse.

\section{Discussion}

Calculations presented at the beginning of this chapter indicated that signal levels of 40 times the threshhold level of the streak camera system were expected to be observed and that distinct lines were expected to be seen. However, as the data in Figure 39 indicated, the signals for both spectrometers were very weak and the traces were "fuzzy" without distinct Iines. Post-measurements ind lcate that the problem of low-signal output was in part due to the impurity of the fluorescer. The problem of indistinct 1 ines was determined to be due to poor spectral and spatial resolution of the system.

$$
\text { Flvorescer conversion efficiency:- }
$$


percent doping of benzophenone. Because the dopant easily leaves the fluorescer, as evidenced by the smell of a doped fluorescer. It was thought by this investigator and others that a 3-year-old, 50- $\mu_{m}$ sample would have lost all its dopant. The dopant is rormally used to enhance the time response of the fluorescer, but in return it drastically lowers the fluorescer's conversion efficiency, contributing to a lower signal output.

The first indication that the presence of

dopant was the cause of the low signals was that postmeasurements of the conversion efficiency showed disagreement with the reported values of pure NElll (Lyons and Lier, 1975; Lyons et al.. 1976).

Direct measurements of the NElll conversion efficiency were done at $0 \mathrm{~K}_{\alpha}$ and $\mathrm{Cu} \mathrm{K}_{\alpha}$ wavelengths. The measured conversion efficiency at $0 \mathrm{~K}_{\alpha}(524.9 \mathrm{eV})$ was $1.74 \times 10^{-3} w / w$, which is the same as the reported value. The value at $\mathrm{Cu} \mathrm{K}_{\alpha}(8047.8 \mathrm{VV})$ was determined to be $1.25 \mathrm{x}$ $10^{-3} \mathrm{w} / \mathrm{w}$ compared with a reported value of $5.0 \times 10^{-3} \mathrm{w} / \mathrm{W}$, a factor of 4 low. These measuremerts are consistent with the hypothesis that there was a dopant concentration gradient in the NElll used. The measurements with the shallow penetrating $0 \mathrm{~K}_{\alpha} \times$ rays gave a characteristic conversion efficlency of undoped NElll, while the measurements with the deeper penetrating $\mathrm{Cu} \mathrm{K}_{\alpha} \times$ rays 
gave a low conversion efficiency consistent with a high dopant concentration.

To further support the gradient hypothesis, measurements were done to determine the time response of two pieces of the $50-\mu_{m} N E 111$ and a $10-\mu_{m}$ sample that was from the same batch as the fluorescer used in the timeresolved experiments. The measurements were done by Steve Lutz of EG\&G, Santa Barbara, using a 50-ps electron pulse to excite the fluorescer and a Varian micro-channel plate as the detector. This kind of measurement is of the bulk properties of the fluorescer: in other words, it gives an indication of the average amount of dopant in the sample. Table 5 shows the results of these measurements, and includes the observed FWHM as well as an estimated unfolded FWHM that accounted for the $0.184-n s$ detector FWHM.

TABLE 5. TIME RESPONSE MEASUREMENTS OF NEIII SAMPLES.

\begin{tabular}{lcc}
\hline Sample & $\begin{array}{c}\text { Observed FWHM } \\
(\mathrm{ns})\end{array}$ & $\begin{array}{c}\text { Unfolded FWHM } \\
(\mathrm{ns})\end{array}$ \\
\hline Pure & - & 1.23 \\
$10 \mu_{\mathrm{m}}$ & 0.876 & 0.855 \\
$50 \mu_{\mathrm{m}}(\mathrm{a})$ & 0.342 & 0.285 \\
$50 \mu_{\mathrm{m}}(\mathrm{b})$ & 0.320 & 0.258
\end{tabular}


These results indicate that the $10-\mu m$ sample has less dopant than the $50-\mu_{m}$ sample but is not pure since its measured FWHM is Iess than that of the pure NEIll sample reported by Lyons and Stevens $(1974)$. The $50-\mu_{m}$ sample has even more dopant, as evidenced by its very fast time response. These measurements support the hypothesis that there is a gradient of dopant in the fluorescer used.

Since benzophenone is made up of the same constituents as NEIIl itself, the conversion efficiency as a function of energy has the same shape for a homogeneously doped sample as that of a pure sample (Steve Lutz, 1986). Therefore, the conversion efficiency as a function of energy of a gradient-doped sample would be the product of a decaying amplitude function of energy, determined by the gradient, and the conversion efficiency of a pure sample.

Spatial Fesolution. During the course of the timeresolved run, distinct spectral lines were not seen in the streak camera data. When the components of the spatial resolution argument presented at the beginning of this chapter were reviewed, the following was found. First of all, the sluorescer was $63.5-\mu_{m}$ thick and not 50 $\mu_{m}$, and secondly, the reported $50-\mu_{m}$ thick interference filter was in actuality 149.1- $\mu_{m}$ thick. Figure 42 shows 


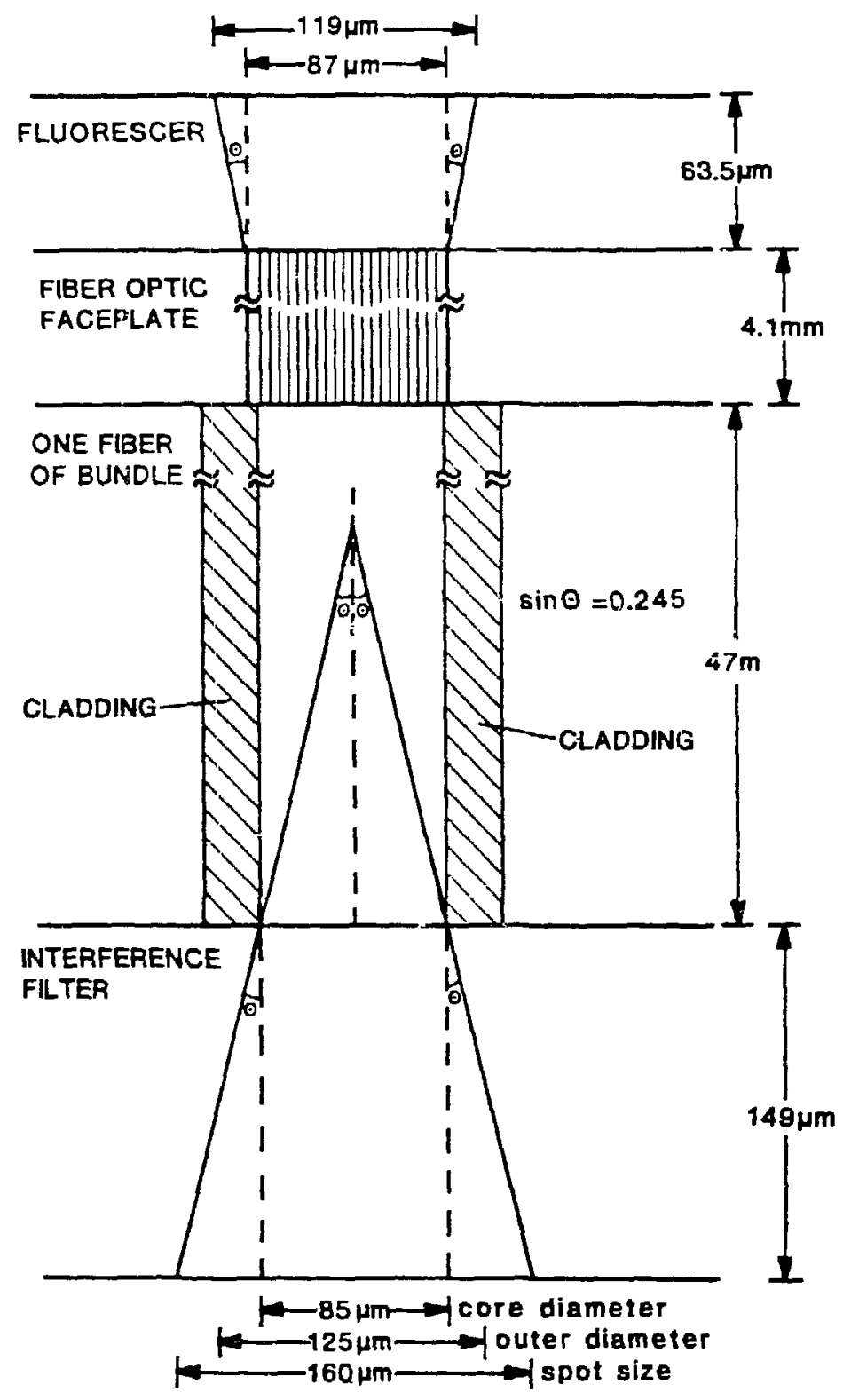

Figure 42. Determination of fpatial resolution of the fluorescer-fiber bunde-interference filter system with measured thicknesses. The spot size on the streak camera's photocathode is greater than the outer diameter of the optical fiber. 
how these thicknesses contribute to the spatial resolution and can be compared with Figure 34. The most significant effect of these thicknesses is that of the interference filter. With a numerical aperture of 0.245 across the 149.1- $\mathrm{m}$ filter, the output spot size is 160 $\mu_{m}$ in diameter. This spot size is greater than the outer diameter of the $f$ iber in the bundle, signifying that the signals from adjacent fibers will overlap. In other words, spatial resolution is ambiguous in this case. This ambiguity and the very low signals on Spectrometer 2 contributed to the indistinct streaks.

Spectral resolution. The experiments were originally designed such that the incidence angles of the multilayers would be nominally 5 and 10 degrees. The 10degcee incidence angle would have given a cleaner line spectrum because there would be a emaller specuiar reflection component of the signal. However, the anode posts that were used during the run obstructed the 1 ine of sight of Spectrometer 2 when the 10-degree multilayers were used.

The theoretical R vs. E curve of the tungetencarbon multilayers used on the time-resolved run is shown in Figure 43. The incidence angle was taken to be 6.25 degrees. Figure 43 showe explicitly the low-energy Epecular : reflection component of the $x$-ray reflectivity.

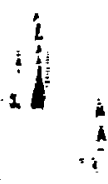




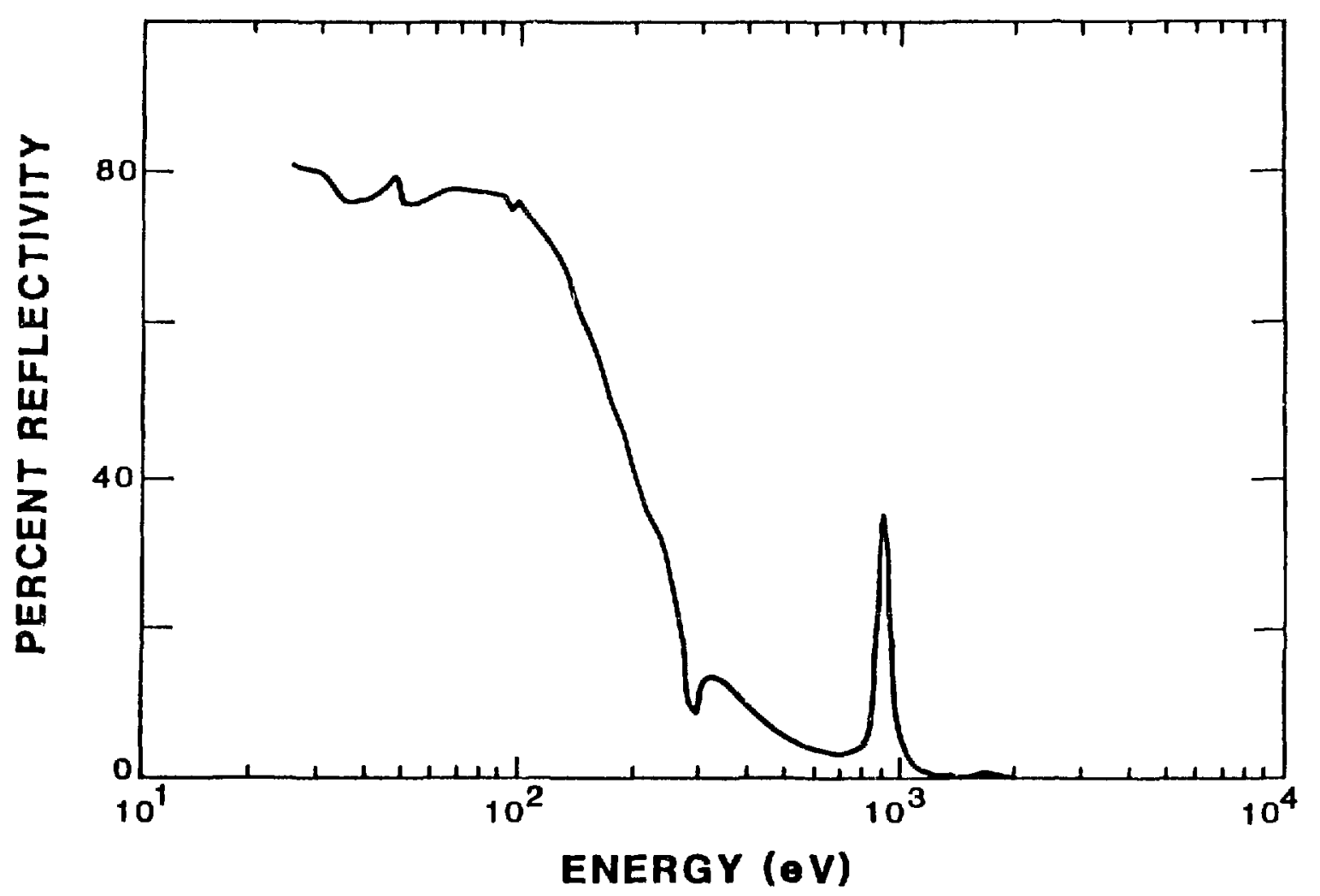

Figure 43. Theoretical R vs. E curve for a tungstencarbon multilayer of $2 \mathrm{~d}=135 \mathrm{~A}$ at an incldence angle of 6.25 degrees. 
For the time-integrated run, distinct spectral lines were observed because the sensitivity of the $x-r a y$ film was so high that it made it possible to use thick filters to attenuate the spectral component of the reflectivity. However, the fluorescer-fiber optic-streak camera detector system was so insensitive that this was not possible, and in fact only very thin aluminum postfilters could be used.

The problems with the fluorescer conversion efficiency, the spatial resolution, and the spectral resolution imply two things. First, absolute comparison of the intensity recorded by the fluorescer-fiber opticstreak camera system and that calculated cannot be made. However, comparison of time history and relative intensity comparisons can still be made. secondly, any information of the rocking curve of the multilayer samples has been washed out with the spatial and spectral resolution problems; however, approximate peak reflection properties can still be determined. 


\section{CHAPTER II -4}

\section{THE INCIDENT SEECTRUM}

Data from the two time-resolved multilayer spectrometers give information relative to the changes in the Spectrometer 2 multilayer reflection properties with respect to the control multilayer in Spectrometer 1 . In order to determine at what flux levels the observed changes occurred, knowledge of the incident $x$-ray spectrum is necessary. Additionally, the incident spectrum must be known in order to calculate the physical deterioration of the multilayer and its corresponding Ieflection properties before a direct comparison with the experimental results can be made. To determine this $x-$ ray flux, it is necessary to extract the spectral characteristics from the data provided by the 6 XRD detectors fielded by R. B. Spielman and S. F. Lopez.

The process of unfolding a spectrum is not an exact science since it is possible to get several answers that are all equally "correct." The inherent time resolution of the multilayer experimental system was $2.4 \mathrm{~ns}$, giving an upper bound for the time steps necessary for the calculations.

The following procedure was implemented to obtain a representation of the incident spectrum. X-ray diode 
traces were obtained from R. B. Spielman. Each XRD trace was then smoothed using the adaptive filtering code AWARE (Hodson et al., 1981). The ratio of the RMS standard deviation of the smoothed curve and the peak of the smoothed curve was taken to be the uncertainty in the XRD signals. By this means, each 5 ignal trace was assigned an uncertainty. For each shot, a table of times for analysis was made. These tables included the times of the peaks observed on the $s i x$ channels as well as additional times at appropriate intervals, depending on the slope of the signal.

The signals for a particular time and their corresponding uncertainties were input into the code SHAZAM (Shirk and lioffman, 1985). For each time, SHAZAM folds an assumed initial spectrum with a set of detector responses and calculates a resultant current signal for each detector. These current values are then compared with the observed current values. The spectrum is then adjusted according to a weighting scheme determined by how far off the calculated current of a detector is from the measured value and its corresponding uncertainty. New currents are calculated for the adjusted spectrum and the process continues.

The XRD responses were taken to be those presented earlier in Figures 17 and 18. The source size was 
approximated from the pinhole photographs taken at 75 degrees from the z-axis of the plasma. The initial "guess" was constructed by using the thin-film bolometer yields to determine a temperature for a blackbody continuum and to determine the line strengths of the two neon resonance lines. This initial guess was constructed in the same way as the incident spectrum calculated in Chapter II-l for the rough heating calculations.

For each time, the code was allowed to rur until the $\underline{x-s q u a r e}$ parameter did not change more than one part in 1000, or until the uncertainties of the channels were satisfied. To ensure that the initial guess did not force the code to give an erroneous result, a second initial guess of a flat spectrum that cut off at 1.3 kev was input into the code. This was to obtain a cross check of whether the trends seen in the results from the first guess were again exhibited. SHAZAM's ability to match detailed spectral features is limited by the detector responses. However, it is very good at matching total yields. An example of the spectrum unfolded by SHAZAM is shown in Figure 44.

\section{Post-Calibration of XRD DetectorE}

The unfolded XRD spectrum was integrated over time and energy to determine the total energy yield to allow 
SHOT 1872

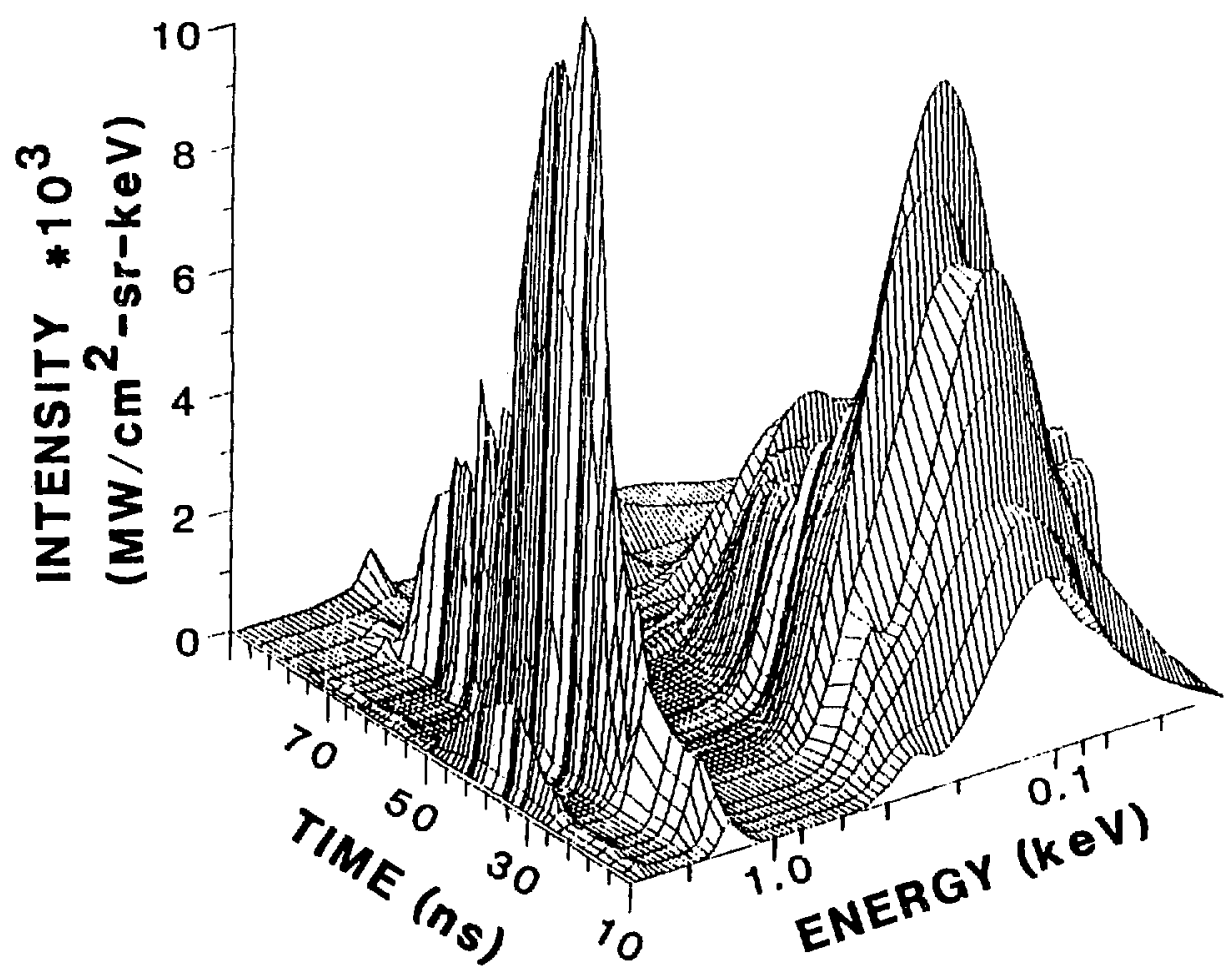

Figure 44. Deconvolved spectrum from XRD data for shot *1872. 
comparison with the reported $75^{\circ}$ bolometer data. After an anistropy factor of 2 was taken into account, there was a large discrepancy between the two yields, much larger than was expected for the difference in acquisition time. After much discussion, it was hypothesized that the photocathode efficiencies of Channels 4, 5, and 7 were in actuality lower than had been previously assumed. A program to measure the photocathode quantum efficiencies and to determine the true response of XRD Channels 4, 5, and 7 was undertaken. In addition, the distances between the detectors and the source were remeasured.

A comparison of the measured quantum efficiencies, $\underline{Y}_{T^{\prime}}$ with those previously assumed is shown in Figure 45. The quantum efficiency for each channel was then extrapolated and interpolated to cover the entire detector energy range.

The distances from the detectors to the source were remeasured and the original distances were found to be incorrect. The distance to the source for Channels 1,2 , and 3 was found to be $152.4 \mathrm{~cm}$ and not $162.56 \mathrm{~cm}$. The accompanying pinhole distance was found to be $83.185 \mathrm{~cm}$ from the source instead of $82.55 \mathrm{~cm}$. The three highenergy channels were also found to be at $152.4 \mathrm{~cm}$ from the source and not $162.56 \mathrm{~cm}$. 


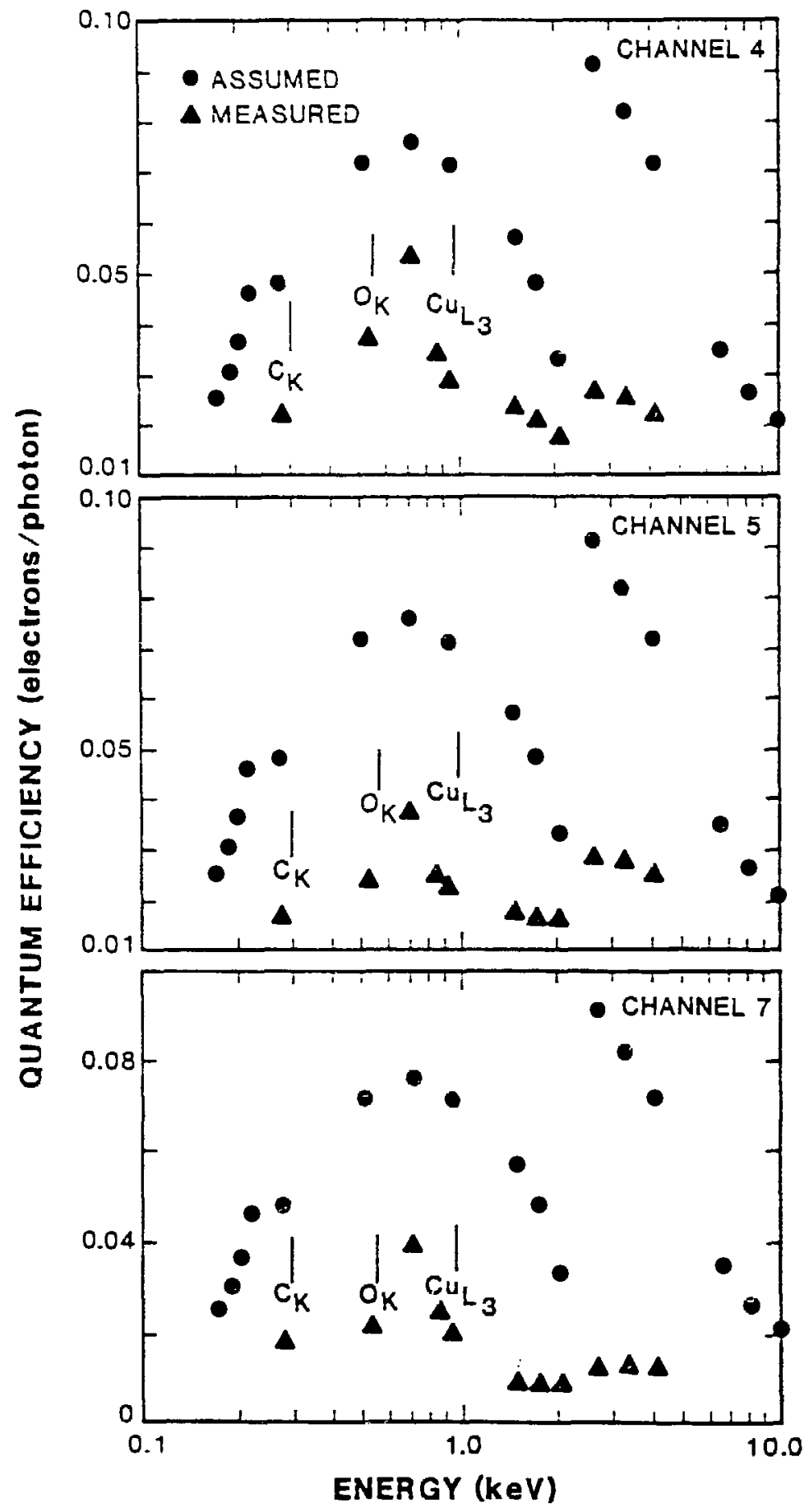

Figure 45. Measured and assumed quantum efficiencles for gold photocathodes of Channels 4, 5, and 7. Straight lines indicate presence of non-gold absorption edges. 
Revised XRD responses were calculated by incorporating the new detector distances into the geometry and the measured photocathode efficiencies. The revieed XRD response and the original response for Channels 4, 5 and 7 are compared in Figure 46.

These XRD responses with the addition of the geometrical factors corrected for the new distances will be referred to as the "post-calibrated" response of the XRD system. The originally assumed detector responses with the corrected geometrical factors will be referred to as the "original" response of the XRD system.

\section{Bolometer and XRD DIscrepancy}

A series of pure neon gas shots was unfolded using one or both XRD responses. The resultant x-ray spectrum was then integrated over time and over the energy windows of the two bolometers in order for a direct comparison of the integrated yields to be made. The XRD yield was multiplied by an anisotropy factor of 2 to normalize the yield to that expected at $75^{\circ}$. These results are shown in Table 6. For low-yield data it is important to note that all the detectors had low signal-to-noise ratios and the discrepancy between the bolometer and XRD $y$ ields is greater.

Shots *1780, *1782, and *1783 were pure neon gas 

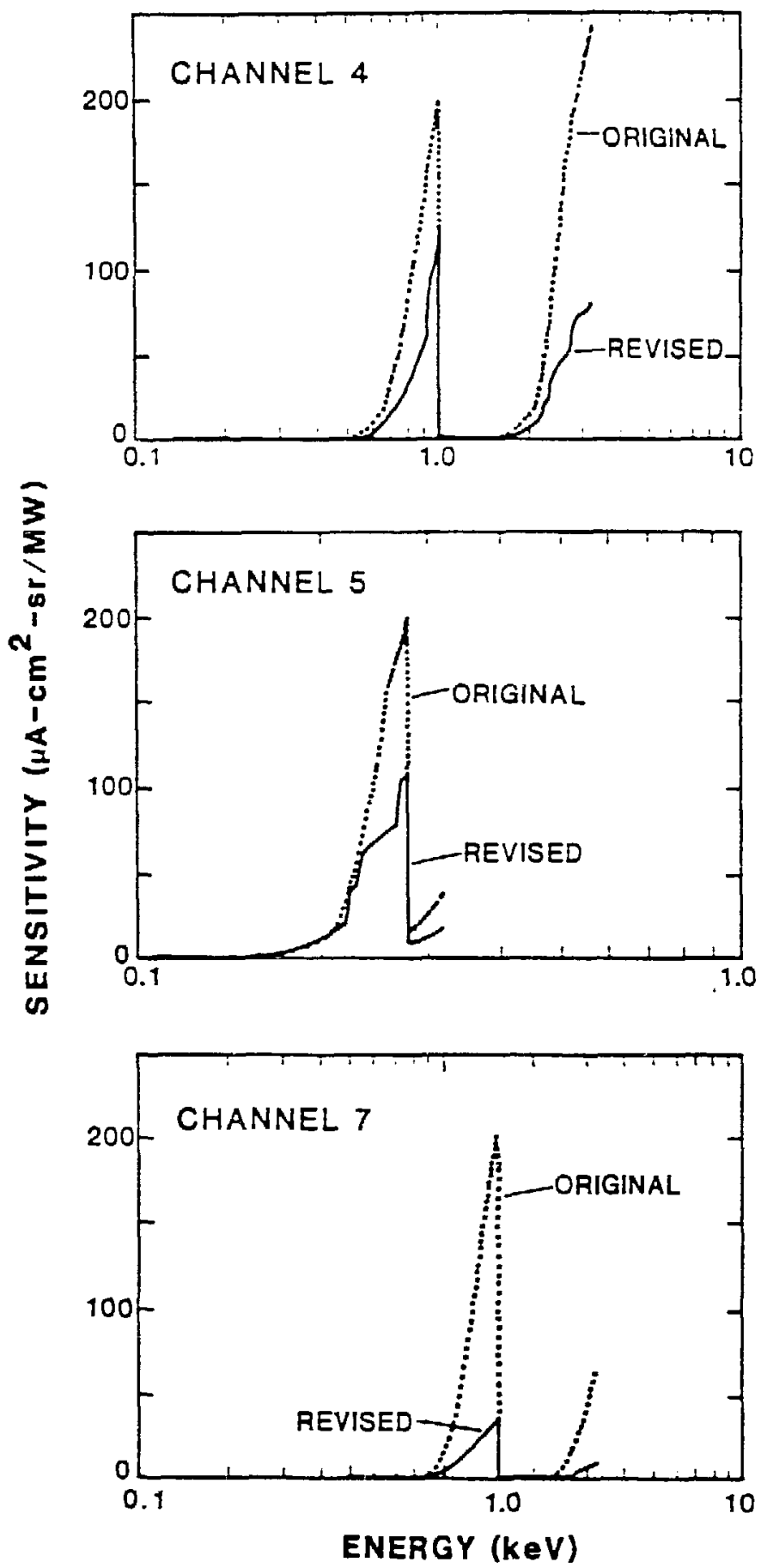

Figure 46. Comparison of original and revised XRD response curves for Channels 4,5 , and 7 . 
TABLE 6. COMPARISON OF FOUR PURE NEON GAS SHOTS.

\begin{tabular}{|c|c|c|c|c|c|c|c|c|c|}
\hline \multirow[b]{2}{*}{$\begin{array}{l}\text { Shot } \\
\text { Number }\end{array}$} & \multirow[b]{2}{*}{$\begin{array}{l}\text { XRD } \\
\text { Response }\end{array}$} & \multicolumn{2}{|c|}{$X R D$} & \multicolumn{2}{|c|}{ Bolometer } & \multirow{2}{*}{$\begin{array}{l}\text { Bolol } \\
\text { XPD } \\
\text { Line } \\
\text { Ratio }\end{array}$} & \multirow{2}{*}{$\begin{array}{l}\text { Bolol } \\
\text { XRD } \\
\text { Total } \\
\text { Ratio }\end{array}$} & \multirow[b]{2}{*}{ Comments } & \\
\hline & & $\begin{array}{l}\text { Line } \\
Y i \in l d \\
(k J)\end{array}$ & $\begin{array}{c}\text { Total } \\
\text { Yield } \\
(k J)\end{array}$ & $\begin{array}{l}\text { Line } \\
\text { Yield } \\
(k J)\end{array}$ & $\begin{array}{c}\text { Total } \\
\text { Yield } \\
(k J)\end{array}$ & & & & \\
\hline 1780 & Original & 3.9 & 6.3 & 8.2 & 26. & 2.1 & 4. 1 & $11 / 84$, Low & Yield \\
\hline 1782 & Original & 17.6 & 32.1 & 13.5 & 37. & .77 & 1.2 & $\begin{array}{l}11 / 84, \quad X R D \\
\text { missing }\end{array}$ & Channel \\
\hline 1783 & Original & 8.0 & 13.4 & 11.4 & 29 & 1.4 & 2.2 & $\begin{array}{l}11 / 84, \text { XRD } \\
\text { missing }\end{array}$ & Channel \\
\hline 1855 & Original & 1.8 & 7.9 & 7.8 & 16.8 & 4.3 & $2 \cdot 1$ & $\begin{array}{l}\text { 3/85, XRD } \\
\text { missing }\end{array}$ & Channel \\
\hline 1855 & $\begin{array}{l}\text { Post- } \\
\text { calibrated }\end{array}$ & $d^{5.4}$ & 8.7 & 7.8 & 16.8 & 1.4 & 1.9 & $\begin{array}{l}\text { 3/85, XRD } \\
\text { missing }\end{array}$ & Channel \\
\hline
\end{tabular}


shots that occurred toward the middle of the timeintegrated cun in the fall of 1984. Shot 1855 was the only purs neon gas shot of the time-resolved run that obtained usable XRD data. Shot 1782 had the highest yield and thus the highest signal-to-noise ratio. For this shot, the total and the line ratios of the bolometer-to-XRD data were of order one when the original XRD response was used for the unfold. This indicates that the original response was appropriate for shots that occurred early in the run.

Shot *1855 gave a better agreement between the bolometer and XRD yields when the post-calibrated response was used. Since all the good time-resolved multilayer data were obtained on shots after 1855 , these shots were all unfolded using the post-calibratsd XRD response.

The discrepancy between the yields of the bolometers and the XRD' 3 increased significantly when neon was imploded upon a target, even though the post-calibrated response was used in the unfold. Table 7 summarizes these flindings for 3 target shots. The discrepancies can be attributed to lower yields and varying amounts of obstruction in the line of sight of the XRD detectors caused by the target holder assemblies. This can be seen in the pinhole pictures taken at 75 degrees from the $z_{-}^{-}$ 
TABLE 7. COMPARISON OF THREE NEON TARGET SHOTS.

\begin{tabular}{|c|c|c|c|c|c|c|c|c|c|c|c|}
\hline \multirow[b]{3}{*}{$\begin{array}{l}\text { Shot } \\
\text { No. }\end{array}$} & \multirow[b]{3}{*}{ Target } & \multicolumn{4}{|c|}{ XRD } & \multicolumn{4}{|c|}{ Bolometer } & \multirow{3}{*}{$\begin{array}{l}\text { Bol'ol } \\
\text { XRD } \\
\text { Line } \\
\text { Ratio }\end{array}$} & \multirow{3}{*}{$\begin{array}{l}\text { Bolol } \\
\text { XRD } \\
\text { Total } \\
\text { Ratio }\end{array}$} \\
\hline & & \multicolumn{2}{|c|}{ Line } & \multicolumn{2}{|c|}{ Total } & \multicolumn{2}{|c|}{ Line } & \multicolumn{2}{|c|}{ Total } & & \\
\hline & & $\begin{array}{c}\text { Yield } \\
(\mathrm{kJ})\end{array}$ & $\begin{array}{l}\text { FWHM } \\
(\mathrm{ns})\end{array}$ & $\begin{array}{c}\text { ield } \\
(k J)\end{array}$ & $\begin{array}{l}\text { FWHM } \\
\text { (ns) }\end{array}$ & $\begin{array}{c}\text { Yield } \\
(\mathrm{kJ})\end{array}$ & $\begin{array}{l}\text { FWHM } \\
(\mathrm{nE})\end{array}$ & $\begin{array}{c}\text { Yield } \\
(\mathrm{kJ})\end{array}$ & $\begin{array}{l}\text { FWHM } \\
(\mathrm{nE})\end{array}$ & & \\
\hline 1860 & $\begin{array}{l}\text { V-doped } \\
\text { foam }\end{array}$ & 1.8 & 18 & $2 \cdot 3$ & 18 & 6.3 & 17 & 17.2 & 12 & 3.5 & 7.5 \\
\hline 1872 & $\begin{array}{l}\text { V-doped } \\
\text { foam }\end{array}$ & 2.2 & 20 & 3.4 & 26 & 10.1 & 18 & 19.5 & 26 & 4.6 & 5.7 \\
\hline 1875 & $\begin{array}{l}\text { Cu-A1- } \\
\text { doped } \\
\text { foam }\end{array}$ & 3.5 & 22 & 4.5 & 25 & 9.6 & 22 & 26.7 & 27 & 2.7 & 5.9 \\
\hline
\end{tabular}


axis, as shown in Figure 47.

\section{Anisotropy}

During an earlier run, an anisotropy was observed for pure neon gas shots that was on the order of 2 in the distribution of the total x-ray energy observed by bolometers at 13.5 and 60 degrees off the z-axis (Hanson, 1984). This reduction in the $x$-ray intensity near the axis was attributed partially to absorption by the presence of a cold column of gas above the imploding pinch. Maximum total x-ray yields were determined by multiplying the yields at 13.5 degrees by a factor of 2 .

The practice of normalizing the near-axis bolometer yielas to the off-axis bolometer by multiplying by $z$ continued into the present runs, even though the off-axis bolometer had been changed to 75 degrees from 60 degrees. The geometry of the detector system used during the experimental runs is shown in Figure 48. This anisotropy factor was incorporated into the calculations of the XRD $x-r a y$ energy yields and has been included in the values reported in Table 7. However, from the pinhole picture shown in Figure 47, It would be expected that the anisotropy factor between the XRD's at 6.75 degrees and the bolometers at 75 degrees would be even greater than a factor of 2 for target shots. 
Figure 47. X-ray pinhole photograph of Shot 1872 showing the target and the "spider web" target holder. The target holder blocks the $x$ rays emitted from the plasma from reaching the diagnostics at angles closest to the z-axis. 


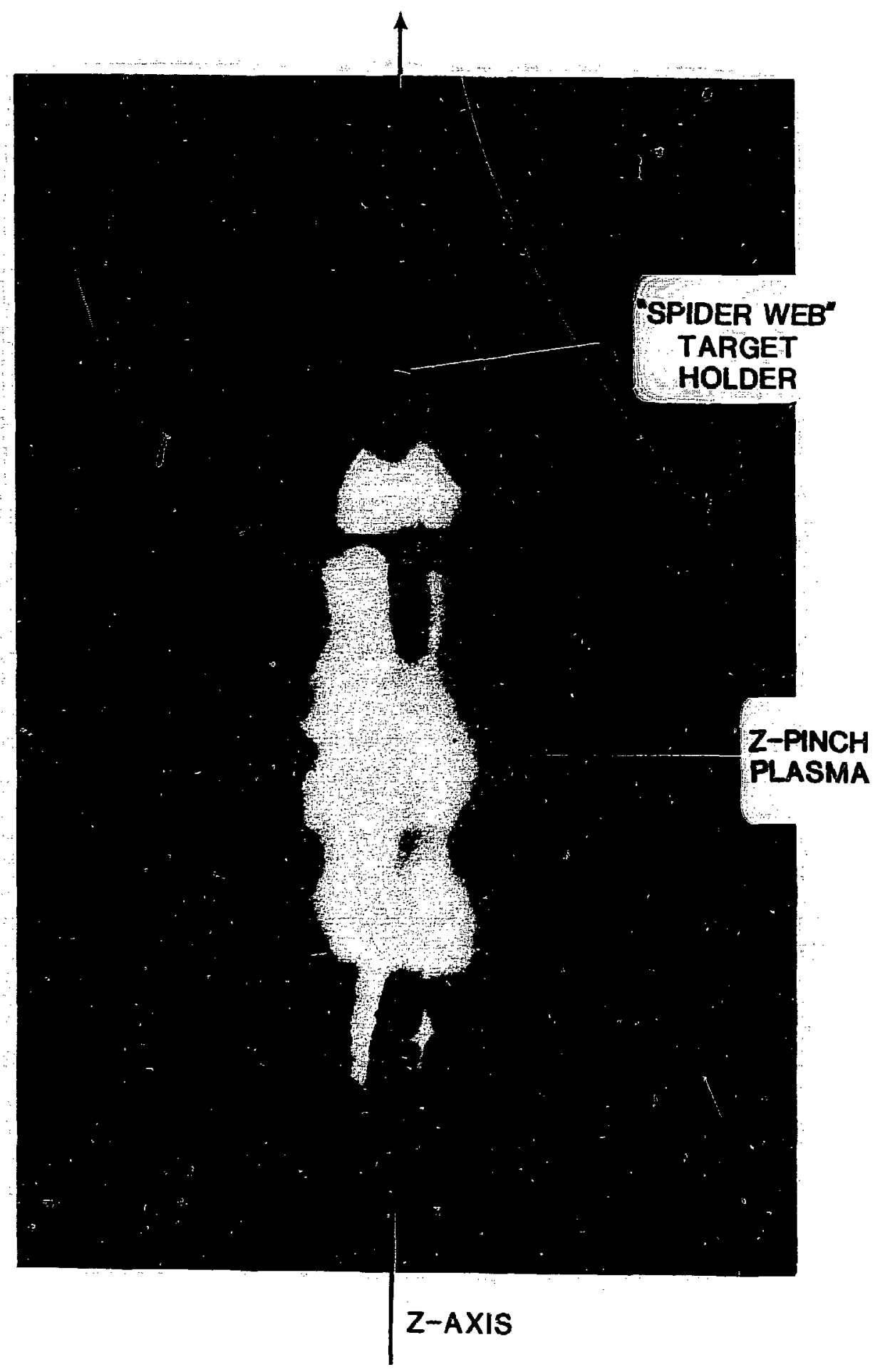


153

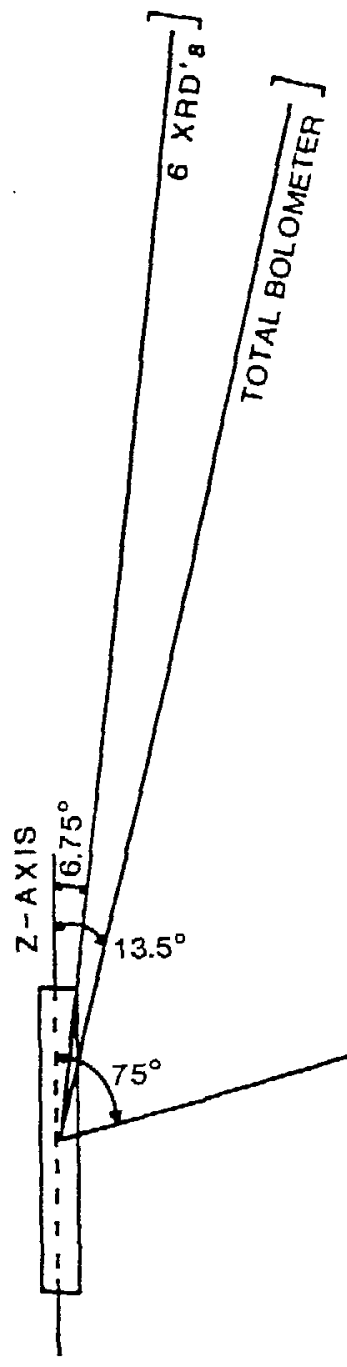

Figure 48. Angular placement of $x$-ray diagnostics on PRATO II. 
To further support the hypothesis of anisotropy. the relationship of the ylelds of two identical bolometers, one at 13.5 degrees and the other at 75 degrees, for target shots on the present run can be investigated. Table 8 summarizes the raw data containing no anisotropy factor. Table 8 indicates that a factor of two anisotropy is adequate for pure neon gas shots, but for target shots it can be a gross underestimate. The XRD-bolometer discrepancy does not seem unreasonable in the light of these numbers.

\section{Similarity of Time History}

Table 7 also shows the FWHM of the bolometer and XRD data. If the unfolded XRD spectrum is integrated only over the bolometer's energy window and plotted as a function of time, the curve traced out is very similar to that of the bolometer sic 11 except that the scale is different. An example $\mathrm{f}$ this is shown in Figure 49 , where the XRD data have been scaled appropriately for Shot *1872. The scaling factor has no anisotropy factor taken into account.

\section{Conversion Efficiency}

Sirce the bolometers and the XRD's were widely separated in yields, it was deemed prudent to determine whether the higher bolometer yields were in fact energet- 
TABLE 8. COMEARISON OF YIELDS OF TWO IDENTICAL BOLOMETERS.

\begin{tabular}{|c|c|c|c|c|c|}
\hline \multirow[b]{2}{*}{$\begin{array}{l}\text { Shot } \\
\text { Number }\end{array}$} & \multirow[b]{2}{*}{ Target } & \multicolumn{2}{|c|}{$\begin{array}{c}\text { Bolometer } \\
(\mathrm{kJ})\end{array}$} & \multicolumn{2}{|c|}{$\begin{array}{r}\text { Anisotropy } \\
\text { Factor }\end{array}$} \\
\hline & & $13.5^{\mathrm{o}}$ & $75^{\circ}$ & $75^{\circ}$ & $113.5^{\circ}$ \\
\hline 1855 & None & 8.7 & 16.8 & & 1.9 \\
\hline 1860 & $\begin{array}{l}\text { v-doped } \\
\text { foam }\end{array}$ & 6.4 & 17.2 & & 2.7 \\
\hline 1872 & $\begin{array}{l}\text { y-doped } \\
\text { foam }\end{array}$ & 5.8 & 19.5 & & 3.4 \\
\hline 1875 & $\begin{array}{l}\text { Cu-Al- } \\
\text { doped } \\
\text { foam }\end{array}$ & 5.5 & 27.1 & & 4.9 \\
\hline
\end{tabular}


SHOT 1872
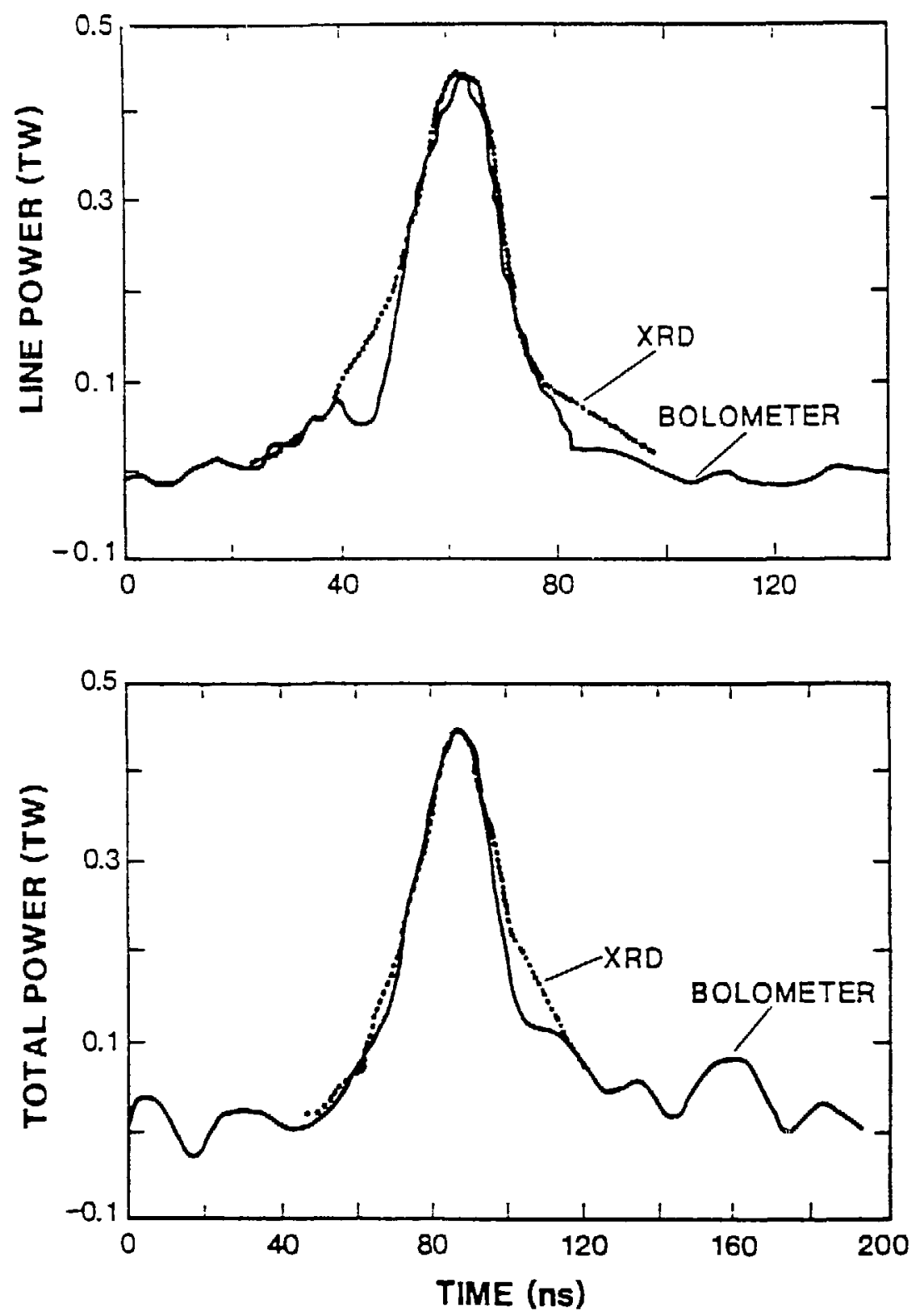

Figure 49. Comparison of 1 ine and total spectral power determined by XRD channels and bolometers for Shot $\$ 1872$. XRD line gignal scaled by.9.9 and the XRD total yield signal scaled by 8.0 . 
ically reasonable. This could be checked by calculating the kinetic energy available in the gas and comparing it with the bolometer total yield to determine a conversion efficiency.

A simple model calculation of the kinetic energy was done as follows. The magnetic pressure exerted onto the outside of a cylindrical shell of gas is assumed to be the only force acting upon the shell, and the pressure is acting upon the entire shell all at once. The shell and the variables to be used in the calculation are depicted in Figure 50. The force equation can be written as

$\frac{m d^{2} r_{2}}{d t^{2}}=\frac{B_{\theta}^{2}(t) A}{8 \pi}$.

where $A=2 \pi r_{2} 1$.

$m=$ pVol $_{\text {init }}$ and

$B_{\theta}=\frac{2 I(t)}{r_{2} c}$

Here Volinit is the initial shell volume, $g$ is the neutral density of the plasma, $c$ is the speed of 1 ight and $\underline{I(t)}$ is the electrical current flowing into the pinch. By substituting Eq. (32) into Eq. (31), the acceleration of the shell, a, is obtained, 


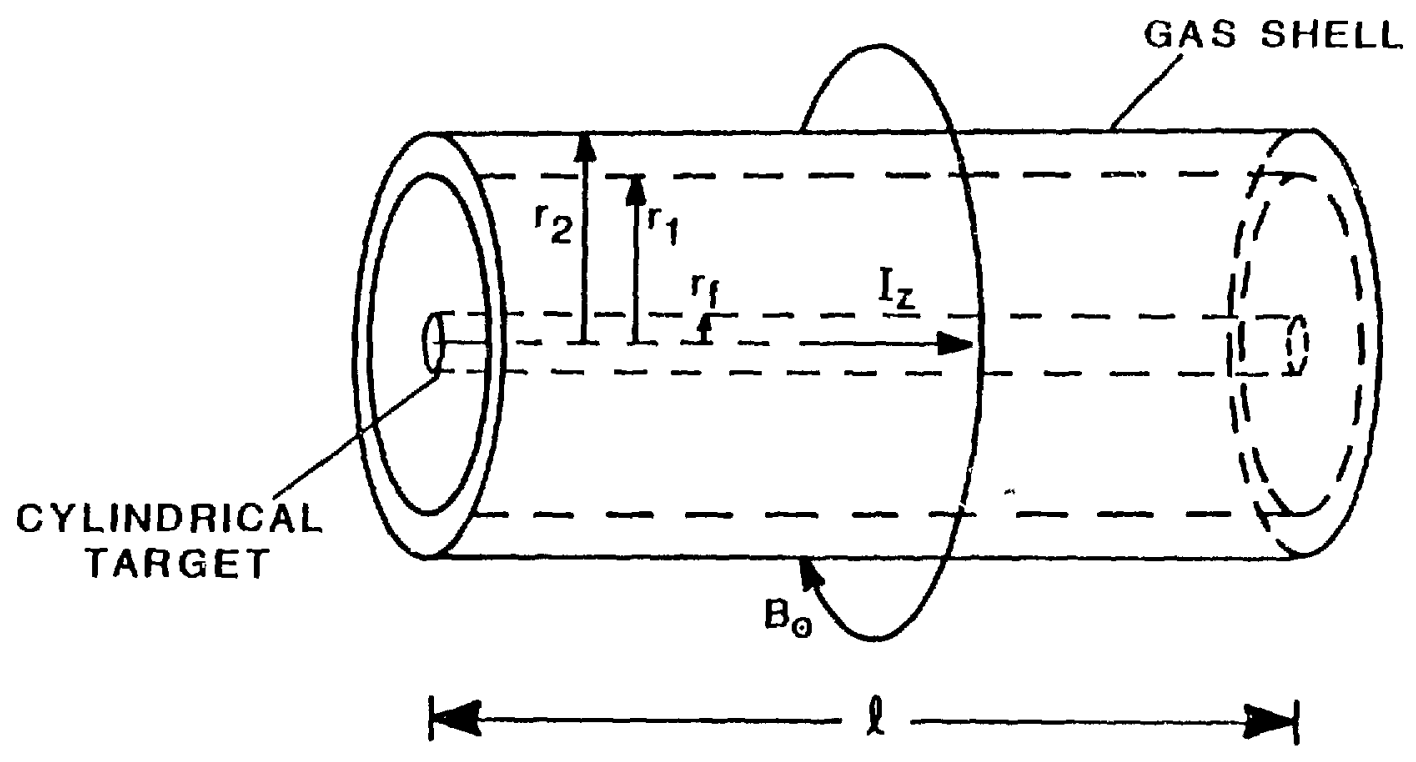

Figure 50. Imploding gas shell of Inner radlus $r_{1}$, outer radius $r_{2}$, and length, 1 , and $c y l i n d r i c a l$ target of radius, $\underline{\underline{E^{\circ}}}$ 
$a(t)=\frac{a^{2} r_{2}}{d t^{2}}=\frac{I^{2}(t)}{\frac{\left(\rho V_{01} \text { init }\right) I_{2}(t) c^{2}}{l}}$.

Adding the initial conditions of velocity and radius. $v(0)=0, r_{2}(0)=r_{\text {init }}$

the equations

$v\left(t_{j}\right)=v\left(t_{j-1}\right)+a\left(t_{j-1}\right)\left(t_{j}-t_{j-1}\right)$

and

$I_{2}\left(t_{j}\right)=r_{2}\left(t_{j-1}\right)+v\left(t_{j-1}\right)\left(t_{j}-t_{j-1}\right)$

$$
+0.5 a\left(t_{j-1}\right)\left(t_{j}-t_{j-1}\right)^{2}
$$

can be solved seguentially in time since the current as a function of $t$ ime was measured by $B$ detectors.

The last two conditions that determine the neutral density are the observed implosion time of the pinch and the final radius of the pinch, $I_{f}$. In the case of the gas imploding onto a target, the implosion $t$ ime that is of interest is the time it takes the gas to implode upon the target, not the implosion of the gas-target systam. The peak of the high-energy XRD channel is indicative of this implosion time. In accordance with this time, the final radius is the outer radius of the target. The neutral density is determined by iterating Egs. (34) and (35) until the final radius is reached at the observed implosion time.

Once the density and the velocity at implosion is 
determined, the kinetic energy can be calculated and compared with the bolometer yields. Figure 51 shows the input current and the calculated radius and velocity curves for Shot \#1872, uring a "best fit" neutral density of $6.5 \times 10^{16}$ particles/cm $\mathrm{cm}^{3}$. This gives a kinetic energy of $65 \mathrm{~kJ}$ arj a conversion efficiency of 30\%. A conversion efficiency of $30 \%$ for this type of pulsedpower machine is reasonable.

Taking all these results into consideration, it was concluded that two incident spectra would be used in the calculation, essentially an upper and a lower value. The lower bound would be given by the unfolded $x-r a y$ spectrum, while the upper bound would be given by the unfolded XRD spectrum scaled such that the XRD spectrum integrated over energy matched the power as a function of time given by the $75^{\circ}$ tota: bolometer. 

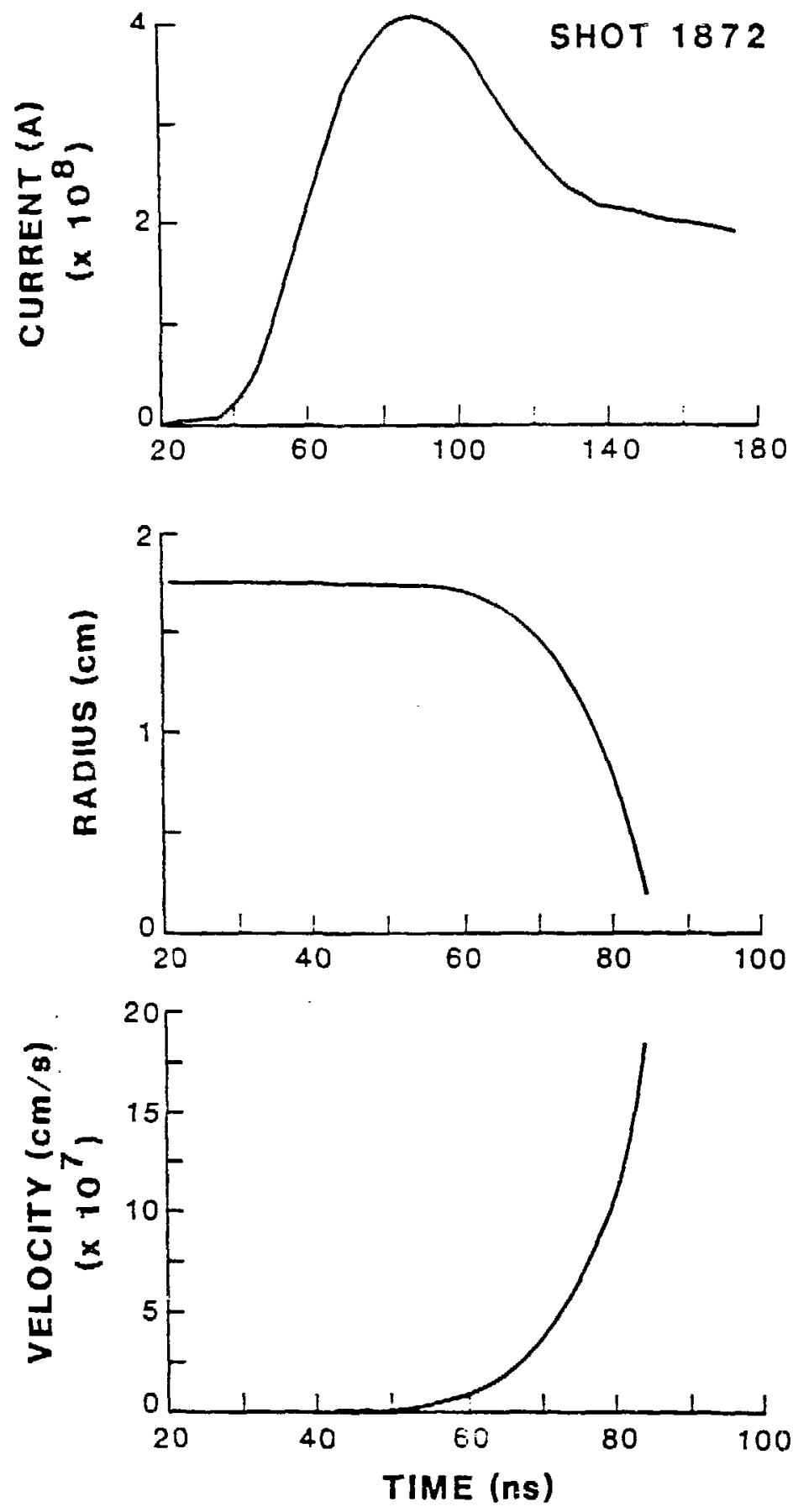

Figure 51. Shot 1872 fnput eurzent and calculated radius and velocity icr a shell with $E=6.5 \times 10^{10}$ particles per cubic centimeter. 
CHAPTER II -5

HEATING MODEL

Once the incident spectral flux is known, the amount of energy absorbed in each layer as a function of time can be calculated. The energy deposition then allows the calculation of a variety of $t$ ime-dependent thermophysical properties for each layer, such as temperature, expansion, and density. The reflection properties as a function of time can then be calculated according to the subsequent physical condition of the mult ilayer.

\section{Energy Deposition}

The first step in modeling the change in the multilayer's reflection properties when it is subjected to high energy $x$-ray $f l u x$ is to determine how much energy is deposited in each layer during each time interval of the $x$-ray pulse. There are two ways to approach this. The first and the more straightforward way is to assume that each multilayer acts as an absorber of the incoming radiatiox. The second is to calculate the absorption according to matrix theory to include all contributions to the absorbed energy of both the forward-and backward-going components of the electromagnetic field 
and not just the effective incident intensity.

In the first method, the calculated reflected spectrum of the whole multilayer is subtracted from the incident intensity, and this difference taken as the effective incident flux on the first layer. The effective incident $i l u x$ is actually determined by taking one minus the reflectivity times the incident flux. The absorptance in the first layer, $\mathbb{A}_{1}$ is as calculated from the equation

$A_{1}=\left(1-t_{1}\right)\langle 1-R\rangle i_{0}$

where $i_{0}$ is the incident intensity, and $t_{1}=\exp \left(-\mu_{i}{ }_{1}\left(\operatorname{REAL}\left(\sin \theta_{1}\right)\right)\right.$.

Here $\mu_{1}$ is the linear absorption coefficient of the first layer, $z_{1}$ is the thickness of the first layer, and $\theta_{1}$ is the grazing angle in the first layer. The resultant intensity incident on the secund layer, $\underline{1}_{2}$ is given by

$i_{2}=(1-R\rangle t_{1} i_{0}$

and so forth as shown in Figure 52. All the above quantities can be a function of energy.

In the second method, the ratio of the difference between the Poynting vector amplitudes at the two interfaces bracketing the layer of interest and the Poynting vector amplitude in the incident medium is calculated. This calculation follows Berning (1963). 


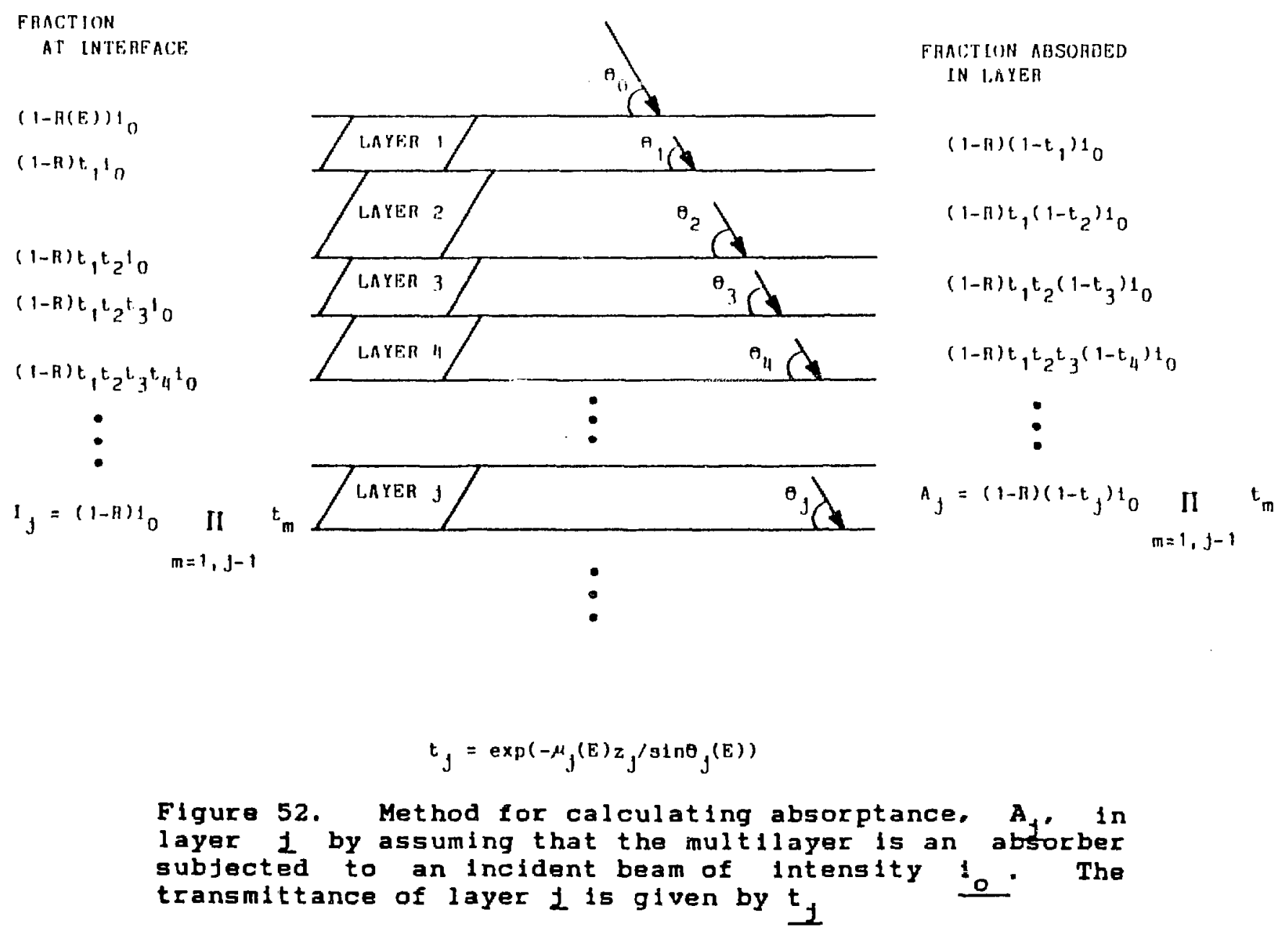


Recalling the results from Chapter I-2, the matrix equation describing the multilayer is

$$
\left[\begin{array}{l}
E_{0}(t) \\
E_{0}(r)
\end{array}\right]=M\left[\begin{array}{c}
E_{m+1}(t) \\
E_{m+1}(r)
\end{array}\right] \text {. }
$$

This equation describes the incident, $E_{0}^{(t)}$, and reflected, $E_{0}^{(I)}$. elsctric field amplitudes in terms of the transmitted and reflected field amplitudes of the exit medium. Figures 8 and 9 of Chapter $I-2$ depict the labeling conventions used for the layers and interfaces. These electric field amp'itudes are related to the total electric and magnetic field amplitudes inside each layer i by the relationf

$$
\left[\begin{array}{l}
E_{j} \\
H_{j}
\end{array}\right]=\left[\begin{array}{cc}
1 & 1 \\
\eta_{j} & -\eta_{j}
\end{array}\right]\left[\begin{array}{l}
E_{j}^{(t)} \\
E_{j}(r)
\end{array}\right] \text {. }
$$

The matrix relation for relating the total electric and magnetic fleld amplitudes of one layer to the precedingf leyer in given by the equation

$$
\left[\begin{array}{l}
E_{j} \\
H_{j}
\end{array}\right]=\left[\begin{array}{cr}
\cos \gamma_{j} & -i / \eta_{j} \sin \psi_{j} \\
-i \eta_{j} \operatorname{sin\gamma _{j}} & \cos \gamma_{j}
\end{array}\right]\left[\begin{array}{l}
E_{j-1} \\
H_{j-l}
\end{array}\right] \text {, }
$$

where $\eta_{1}$ and $I_{1}$ are the effective index of refraction and phase thickness of layer $\mathcal{L}$; that 15 ,

$\eta_{f}=n_{f} /$ Eing, for ' $p$ ' polarization. or

$$
\eta_{j}=n_{j} \sin _{j} \text { for 'E' polarization. }
$$

and $\nabla_{j}=\frac{2 \pi}{\lambda}\left(n_{j} E i n \theta_{j}\right)$.

Since the electric and magnetic field amplitudes are known, the effective Poynting vector amplitude, 
REAL[ $\left.\mathrm{E}_{j} \mathrm{H}_{j}^{\star}\right]$, at each interface can now be calculated. The ratio of the difference between the two Poynting vector amplitudes at each interface surrounding the layer and the incident intensity on the upper side of the two interfaces is defined as the fractional absorptance, $A_{i}$ of that layer. In other words, $A_{j}$ is expressed by the equation

$A_{j}=\frac{\left.\left.4\left(\operatorname{REALCE} E_{j} H_{j}{ }^{\star}\right]-\operatorname{REAL[} E_{j-1} H_{j-1}{ }^{\star}\right]\right)}{\Omega_{0}\left|E_{0}+H_{0}\right|^{2}}$.

The equations (40) and (41) together give the recursion formula for the $A_{j}^{\prime} s$. To get the energy deposited into the layer the $A_{j}$ is multiplied by the incident spectral flux.

It is instructive to compare the results of the two methods. The $\underline{R}$ v. E curve for a particular 1 such as that given in Figure 53 has three distinct regions of interest the specular reflection region, the Bragg diffraction region, and that region which is far from the other two regions. Figures 54 through 56 compare the results for each method for the three reglons for a $21-$ layer, tungsten-carbon multilayer of $2 \mathrm{~d}=100 \mathrm{R}$ including the silicon substrate assuming 5 polarization. The absorptance is plotted as a histogram because no information of how the absorbed energy is distributed within the layer is calculated, only the total absorptance for that particular layer. 


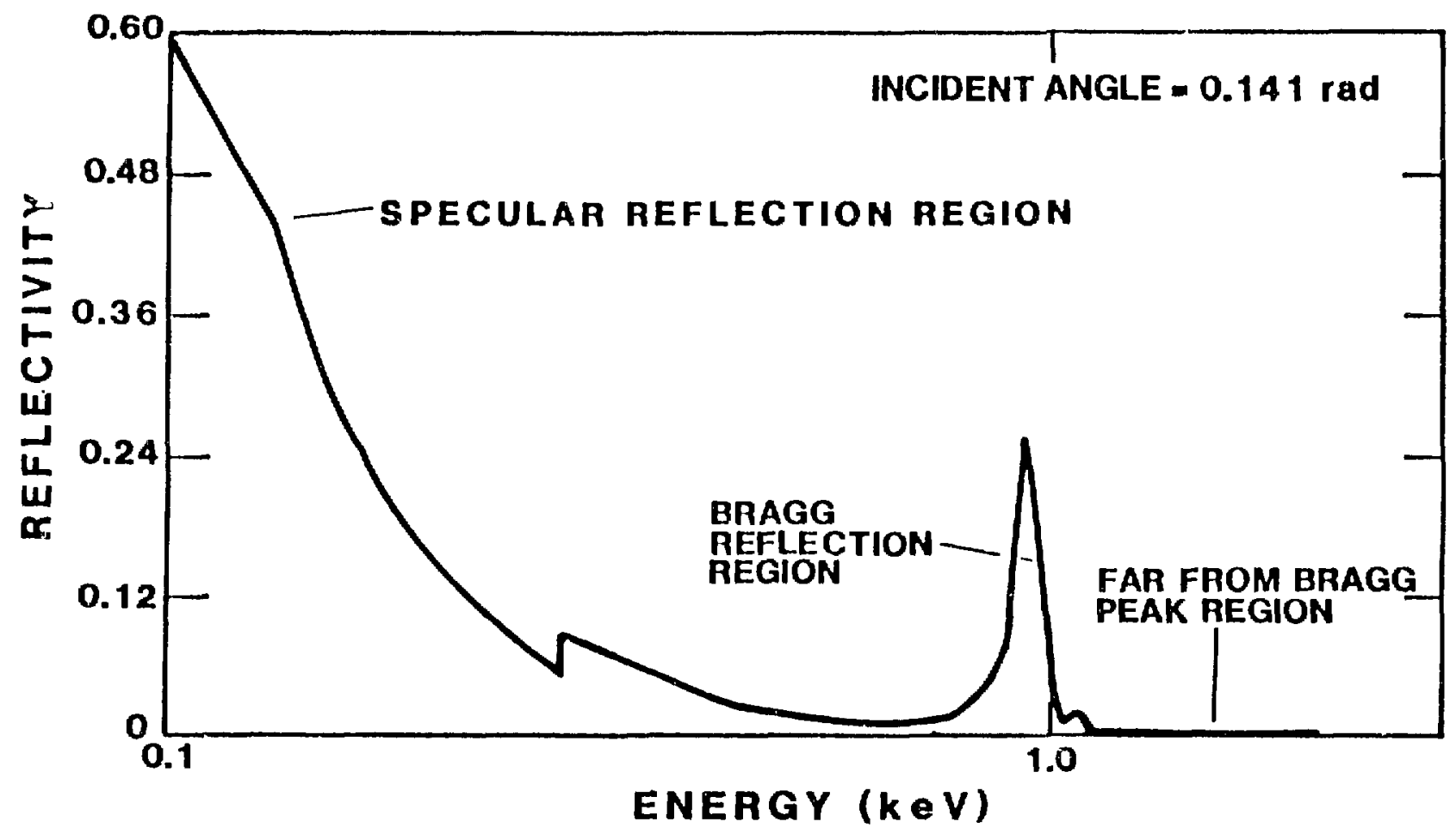

Figure 53. Reflectlvity versus photon energy curve for a 21-layer, tungsten-carbon multilayer. 


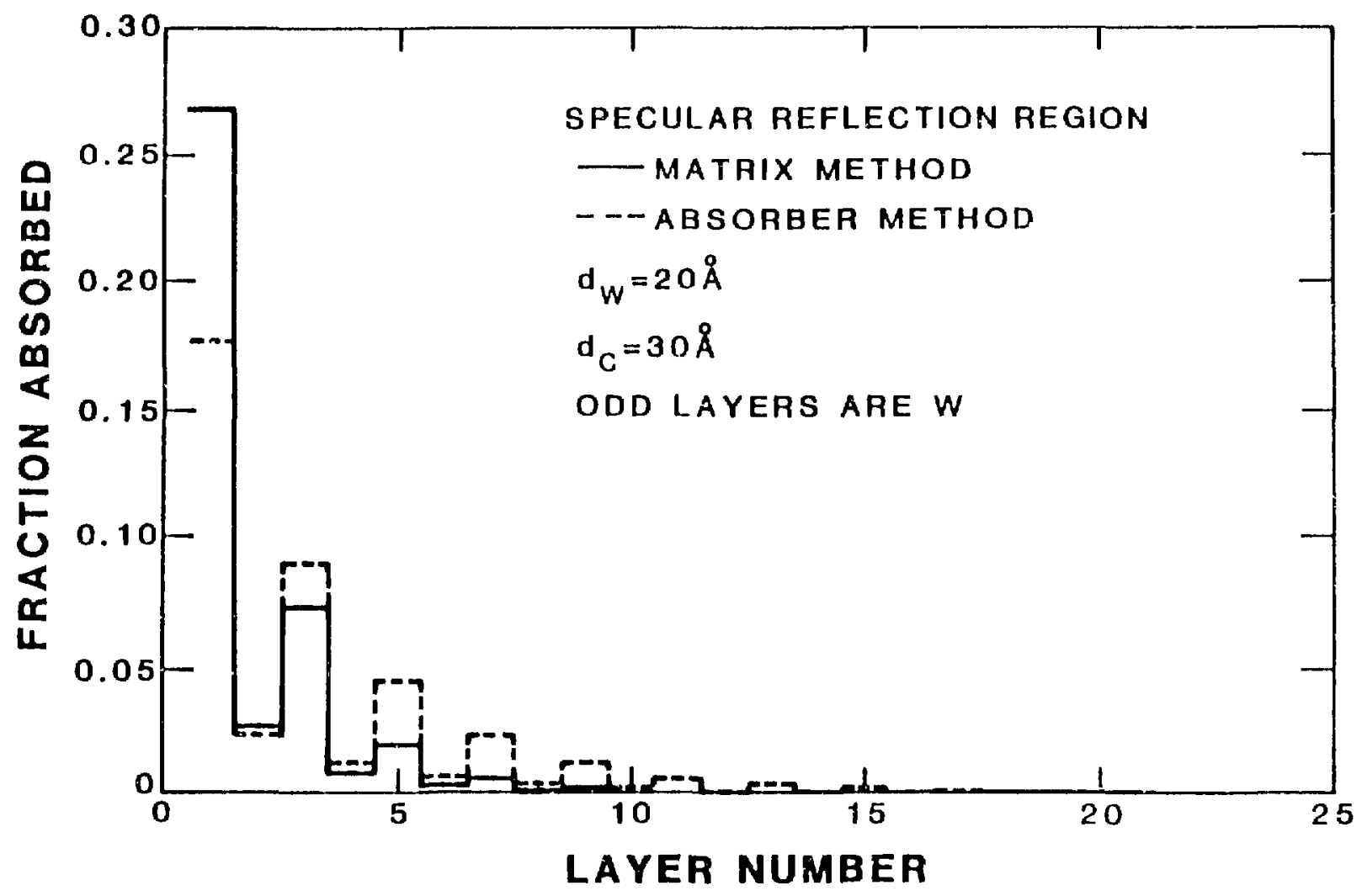

Figure 54. Absorptarice calculated by the absorber and Poynting amplitude methods for the specular reflection reglon. 


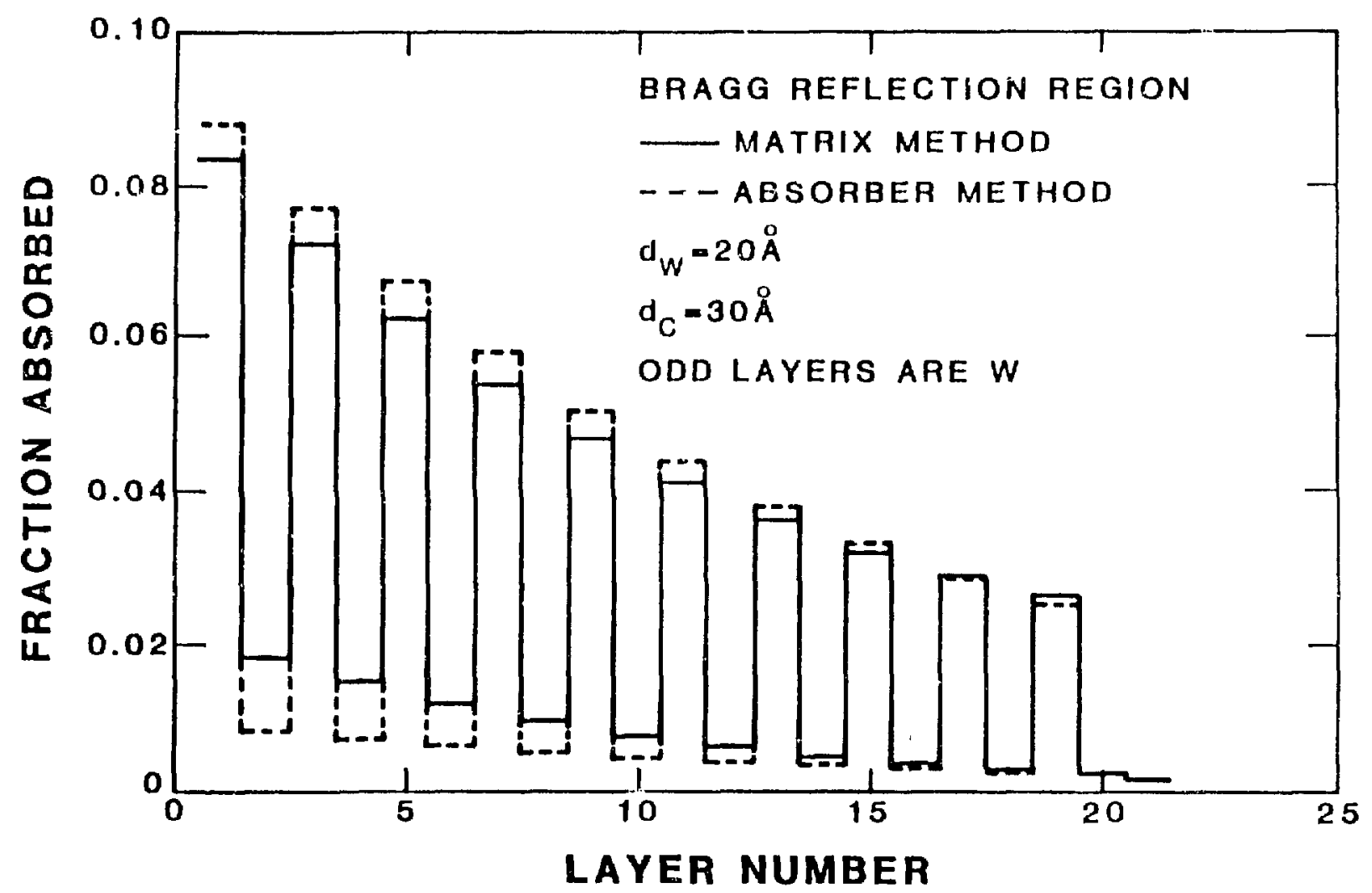

Figure 55. Absorptance calculated by the absorber and the Poynting amplitude method for the Bragg reflection region. 


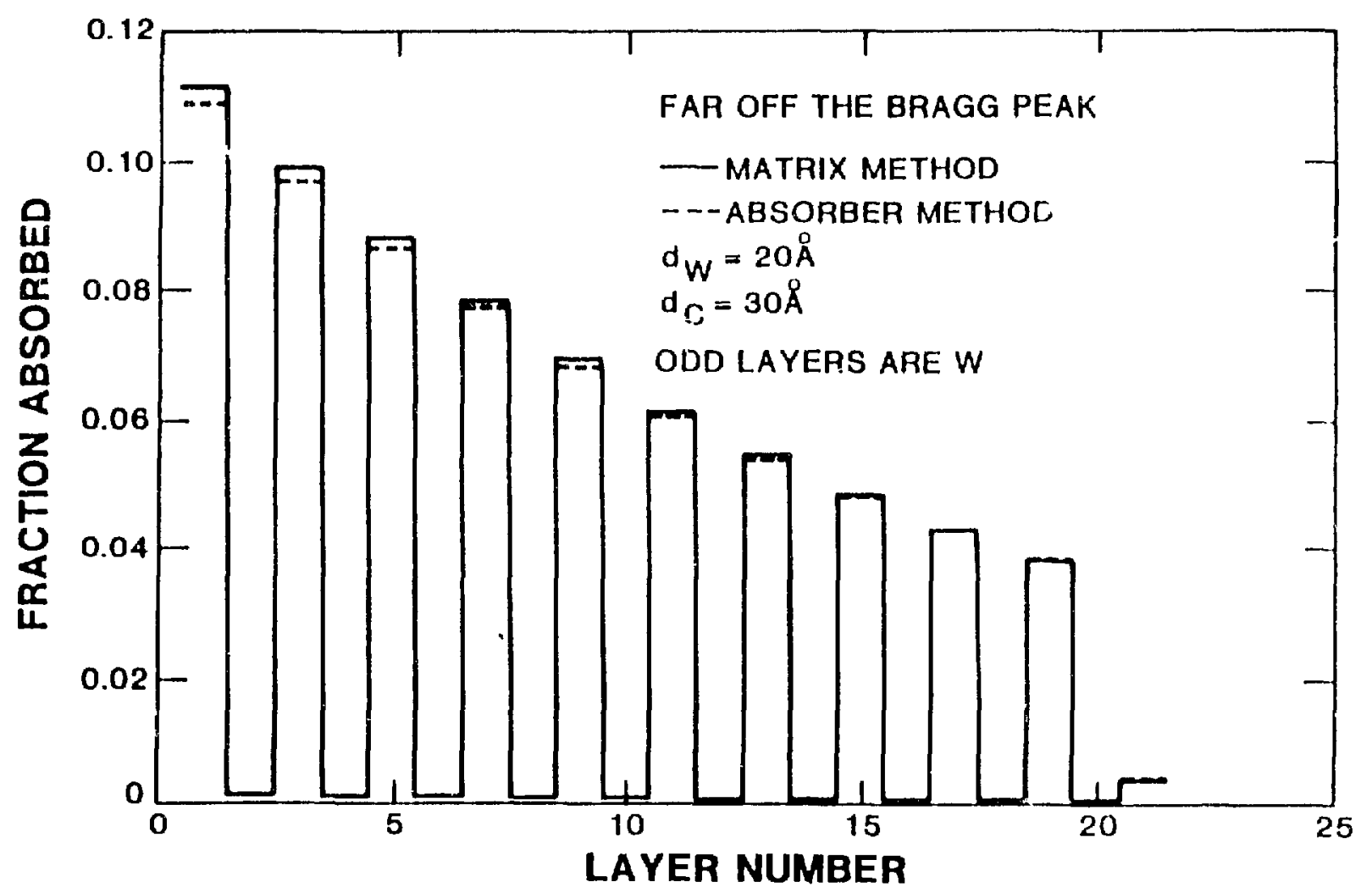

Figure 56. Absorptance calculated by the absorber and the Poynt ing amplitude method for the region far from the Bragg and specular reflect fon regions. 
Figure 54 shows that in the specular reflection region more of the energy is absorbed in the first layer of tungsten by using the Poynting vector amplitude method than by using the absorber method. Thisis reasonable since in specular reflection there is a skin depth phenomenon (Henke, 1981a) not taken into account by the absorber method.

Figure 55 compares the two methods in the Bragg diffraction region. More absorption occurs in the top layers of tungeten in the absorber calculation than in the Poynting vector amplitude calculation.

Figure 56 shows the region far from the specular reflection region and far from the Bragg peak. It is encouraging to note that, as was expected, there is little difference, if any, between the two theories in this region.

Overall there is not a great difference between the results of the two methods. This is important since all of the experimental design calculations that were performed to determine the experimental parameters used the absorber method. However, the Poynting vector amplitude method was incorporated into the detailed modeling because of the large low-energy specular component of the spectral flux. 


\section{Thermophysical Properties}

The enthalpy from room temperature for each layer, if conduction is ignored, is determined by integrating the deposited energy in the layer over the spectral range. By neglecting conduction, an upper limit for the heating can be obtained. Values of enthalpy as a function of temperature were taken from Hultgren et al. ( $1973 \mathrm{a}, \mathrm{b}$ and c) for tungsten, carbon (graphite), and silicon. Enthalpy values through $4200^{\circ} \mathrm{K}$ for carbon, through $6000{ }^{\circ} \mathrm{K}$ for tungsten, and through $3600^{\circ} \mathrm{K}$ for silicon were used. The values for enthalpy were inverted to give temperature as a function of enthalpy. The inverted values were then fit with polynomials for different temperature regions, depending on the materials. This allowed for direct computation of the temperature at each layer.

With the temperature known at each time step, the corresponding expansion of the layers could be determined. Thermal expansion coeffisients as a function of temperature were from Touloukian et al. (1975) for glassy carbon and silicon and Touloukian et al. (1970b) for tungsten. Expansion coefficieris through $3600^{\circ} \mathrm{K}$ for tungsten, through $2600^{\circ} \mathrm{K}$ for carbon, and through $1600^{\circ} \mathrm{K}$ for silicon were used. These were also fit with polynomials. The thermal expansion coefficient 
polynomials were then used to calculate the change expected in the d-spacings of the layers in the multilayer.

The subsequent change in density of the layer due to the expansion was calculated by taking the original density, multiplying by the original thickness of the layer, and dividing by the expanded thickness. This assumes that the expansion occurrad only in the zdirection. The density was required to calculate the index of refraction of the layer.

\section{Multilayer Reflection Properties}

To determine the reflectivity of the multilayer as a function of intensity and angle during the $x$-ray pulse, it would normally be necessary to partition the incident angle as well as time. However, from the aiscussion in Chapter II-3, the naon resonance 1 ines were not distinct above the specular signal; therefore. the problem of modeling the temporal changes of the $x$-ray reflection properties reduces to a one-dimensional problem of the reflected flux integrated over energy at the central angle of incldence versus time.

To put this into practice, the Rvs. E surve is calculated for each time step according to the calculated physical condition of the multilayer. A simplified flow 
chart showing the logic of the code is shorn in Figure 57. The reflected $f l u x$ is determined by multiplying the $\underline{R}$ Us. E curve by the incident flux as a function of energy determined by the XRD-bolometer data. This reflected flux is then multiplied by the fizter transmission and the fluorsscer corversion efficiency as a function of energy and then integrated. This integrated response is then related to the density observed on the streak camera ilm. The computer program is giver in sppendix $B$. 


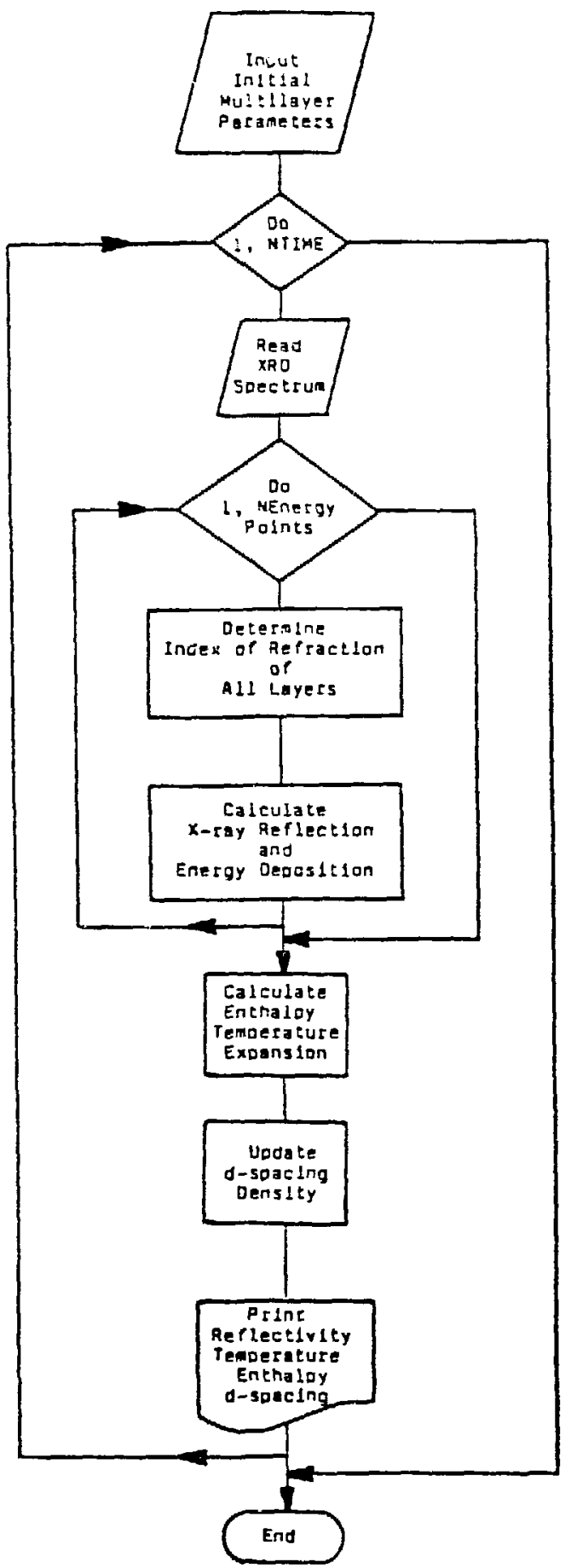

Figure 57. Simplified flow chart of the combined multilayer and heating computer code. 
CHAPTER II-6

ANALYSIS

The two best shots from the time-resulved experiments were chosen to be examined closely. They were Shots $* 1863$ and 1972 .

\section{Shot 1863}

Out of the nine shots for which time-resolved data were obtained, Shot \# 1863 had the largest total yield. For Shot 1863 the tungsten-carbon multilayers used were set at an angle of $6.3^{\circ}$. OVLA-130-B-1-III was used in Spectrometer 1 and 0.5 of OVLA-130B-1-V was used in Spectrometer 2. Only $196.5 \mu \mathrm{g} / \mathrm{cm}^{2}$ Al postfilters were used on both spectrometers. Shot *1863 was also a pure neon shot and its spectral flux should be the easiest to determine; however, four of the $s i x$ XRD's went off scale, preventing deconvolution of the spectrum. An attempt was made to determine whether the spectral shape of the incident $f l u x$ as a function of $t$ ime could be determined from other pure neon gas shots. The spectral flux of the four neon gas shots, $1780, \$ 1782, \# 1783$, and \#1855, were deconvolved from their corresponding XRD data. These shots were previously tablulated in Table 6 of Chapter II-4. The resultant spectra were very dissimilar, making 
it difficult to construct an incident spectrum to be used to model shot \#1863. This shot-to-shot variation can be seen in Figure 58, where the time-integrated data from the KAP spectrograph for three pure neon gas shots is shown. Detailed modeling was therefore not attempted. Information on the destruction of the multilayer can be obtained by comparing the time history of the total yield bolometer data with the smoothed, compressed multilayer spectrometer data as shown in Figure 59. The ieft vertical axis is in terawatts ( $T W$ ) radiated in $4 \pi \mathrm{sr}$ corresponding to the bolometer data, and the right vertical axis is erg/cm deposited on the streak camera film. Zero time is arbitrary and set to the beginning of the streak of Spectrometer 1 . The bolometer time was shifted such that the bolometer peak matched the peak of Spectrometer 1 . Spectrometer 2 data were shifted with respect to spectrometer 1 to account for the time of flight difference between the two. The averaging for the compressea streaks for the two spectrometer signals was over a different number of pixels. Spectrometer 2's signal was averaged over 4 times as many pixels as Spectrometer l's, resulting in a lower average exposure. If Spectrometer 2's signal is scaled accordingly, as shown in Figure 60, it follows both the bolometer and spectrometer 1 signals, dips a little, and then decays 

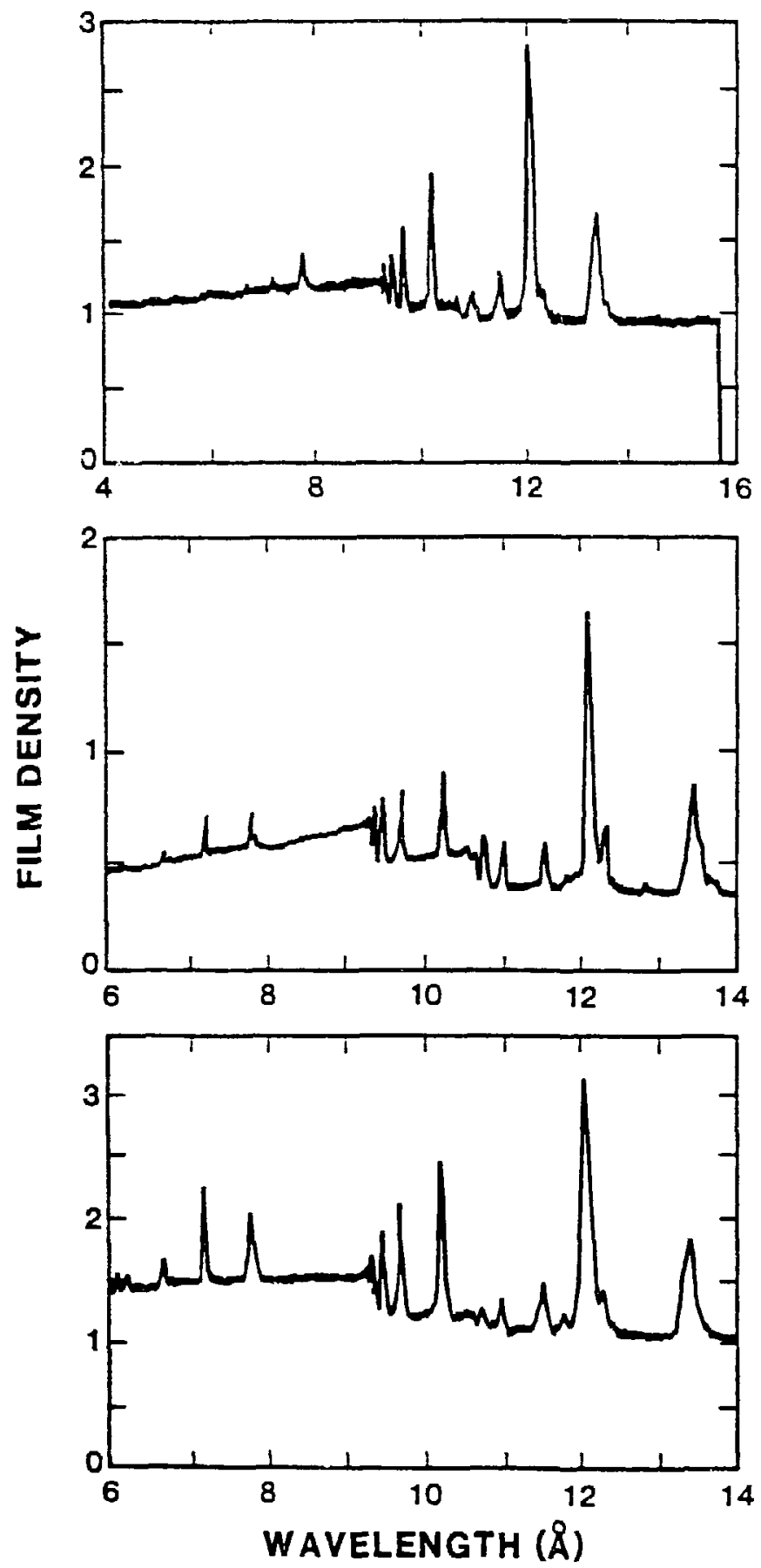

Figure 58. Spectra taken with the KAP spectrograph fielded by $M$. A. Palmer for three neon gas shots showing the non-repeatability. 


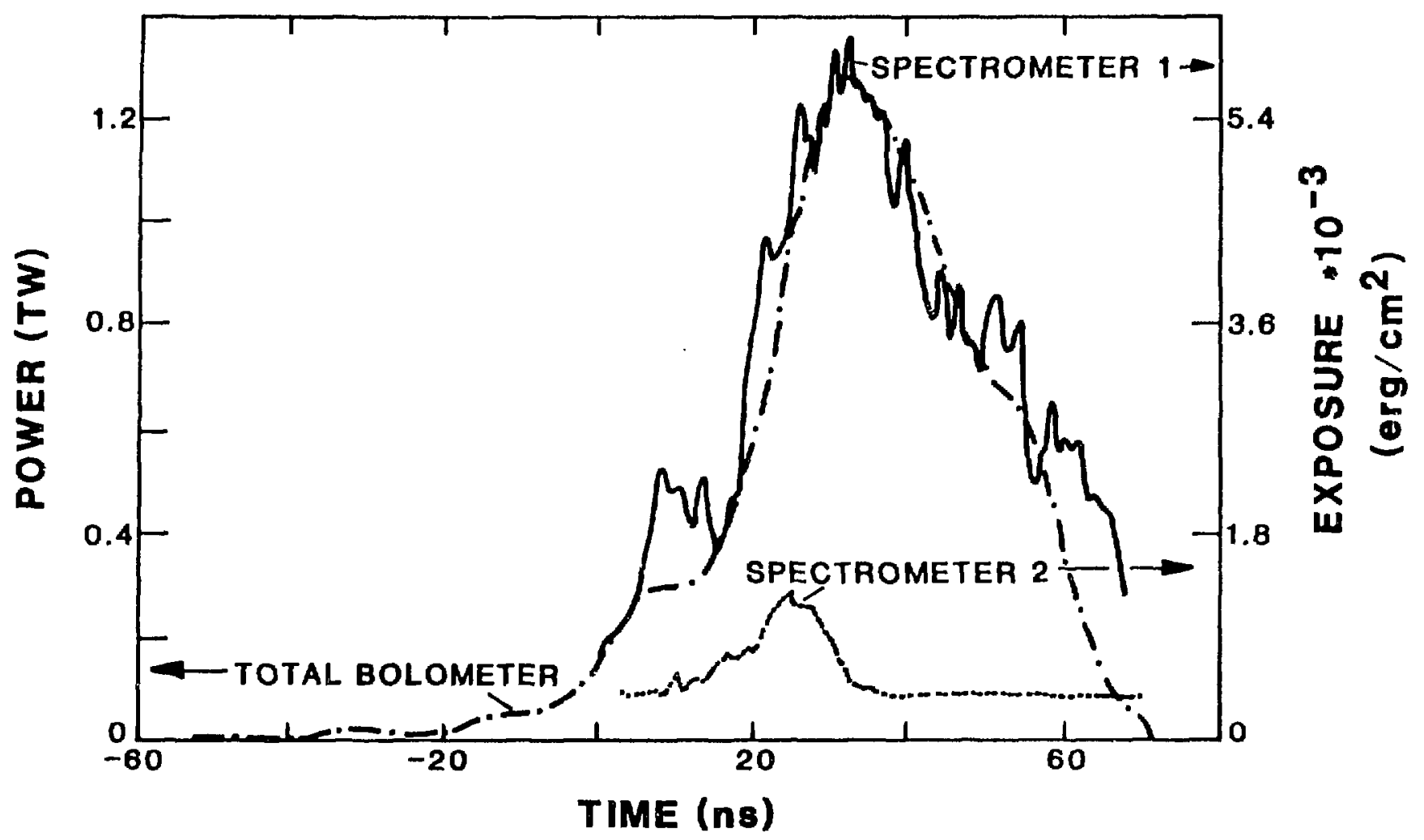

Figure 59. Total bolometer and multilayer spectrometer data for shot 1853 . The left-hand vertical axis is the total Power determined by the bolometer ignal in $4 \pi$ Gr. The right-hand vertical axis is the exposure on the streak canera $111 \mathrm{~m}$. 

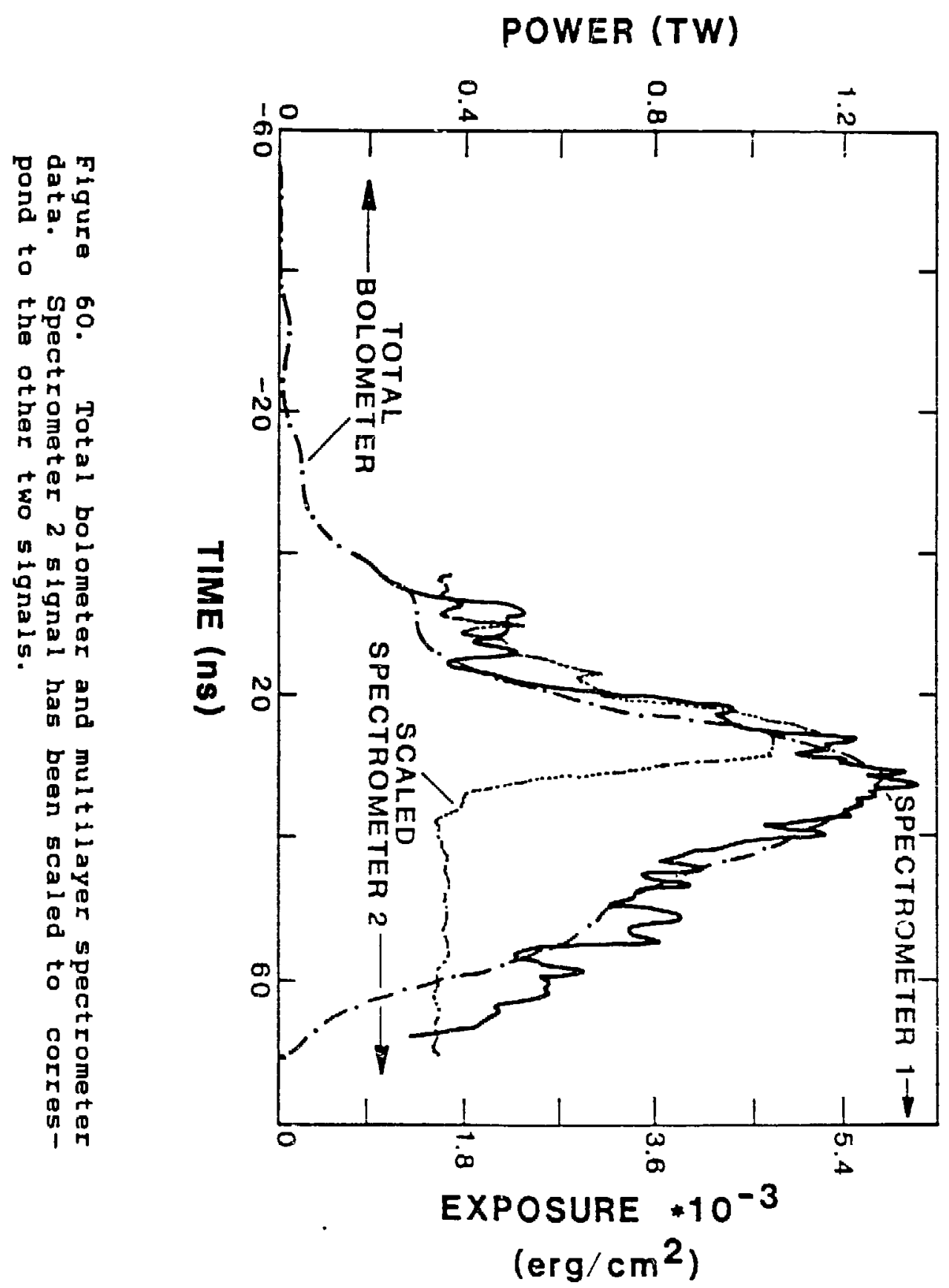
rapidly at $\sim 28 \mathrm{~ns}$.

As can be seen in Figure 59, the control spectrometer signal. Spectrometer 1, has the same timing as the bolometer data. The streak camera data is noisy. Spectrometer 2's sigral, once it gets out of the noise, decays on the rise of the two other signals. This indicates that the multilayer's reflectivity was decaying early, before the peak of the pulse. The power level determined from the bolometer at the start of the constant decay of the Spectrometer 2 data around $\sim 28$ ns is $\sim 1.2$ TW radiated in $4 \pi 5 \pi$. This is equivalent to 420 $\mathrm{MW} / \mathrm{cm}^{2}$ at the multilayer of Spectrometer 2 .

The total integrated energy as a function of time that was produced can be determined by integrating the total yield bolometer signal. Figure 61 shows the integrated bolometer signal superimposed on the streak camera data. The total yield was $42.2 \mathrm{~kJ}$ into $4 \pi \mathrm{sr}$, which is equivalent to a total dose of $15.0 \mathrm{~J} / \mathrm{cm}^{2}$ at the multilayer of spectrometer 2. The decay of the multilayer reflection at $28 \mathrm{~ns}$ corresponds to $\sim 14.9 \mathrm{~kJ}$ into $4 \pi$ sr or a dose of $5.27 \mathrm{~J} / \mathrm{cm}^{2}$ at the multilayer of Spectrometer 2 .

\section{Shot $\# 1872$}

Shot 1872 had good quality multilayer data as well 


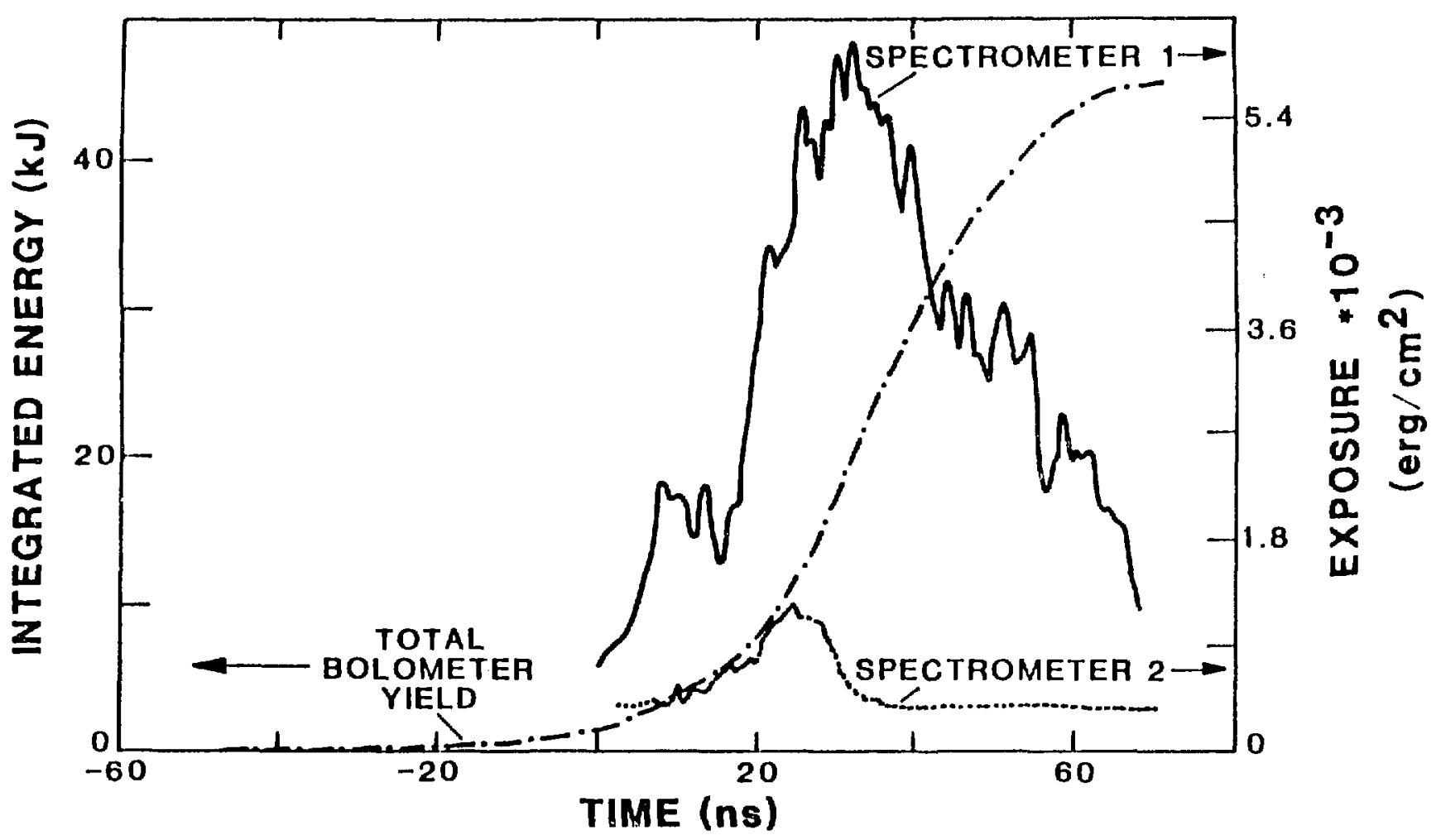

Figure 61. The integrated total bolometer signal and the data from the two multilayer spectrometers for Shot 1863 . The left vertical axis gives the total integrated energy. The right axis glves the exposure on the streak camera film for the two compressed streaks. 
as data from 5 out of the 6 XRD'E. Channel 4 XRD failed. The multilayer used in Spectrometer 1 was OVLA 130B-1-III and the multilayer used in Spectrometer 2 was 0.5 of OVLA13OB-1-IV, at an incidence angle of 6.26 degrees. Again only post filters were used and they were 123 $\mu \mathrm{g} / \mathrm{cm}^{2}$ of Al for both spectrometers.

The total yield bolometer data and the smoothed compressed multilayer spectrometer data are shown in Figure 62. The left axis is the total power radiated by the plasma into $4 \pi \mathrm{sr}$ as detected by the bolometer. The right axis gives the averaged ergs $/ \mathrm{cm}^{2}$ on the streak camera film. Again Spectrometer l's signal is a lot higher than spectrometer 2's. This is again due to the averaging process and the background levels. Spectrometer 2 follows the other two signals, possibly dips, and then decays rapidly, indicating the degradation in the reflectivity of Spectrometer 2's multilayer. At the start of the continual decay at 19.5 ns the power level was $0.4 \mathrm{TW}$ into $4 \pi \mathrm{sr}$ or $141 \mathrm{MW} / \mathrm{cm}^{2}$ at Spectrometer 2 's multilayer.

The integrated energy of the bolometer as a function of time is superimposed on the multilayer data as shown in Figure 63. At 19.5 ns the plasma had produced an integrated energy of $\sim 5.5 \mathrm{~kJ}$ into $4 \pi \mathrm{sr}$ which gives a dose of $2 \mathrm{~J} / \mathrm{cm}^{2}$. Since data for 5 out of the 6 XRD 


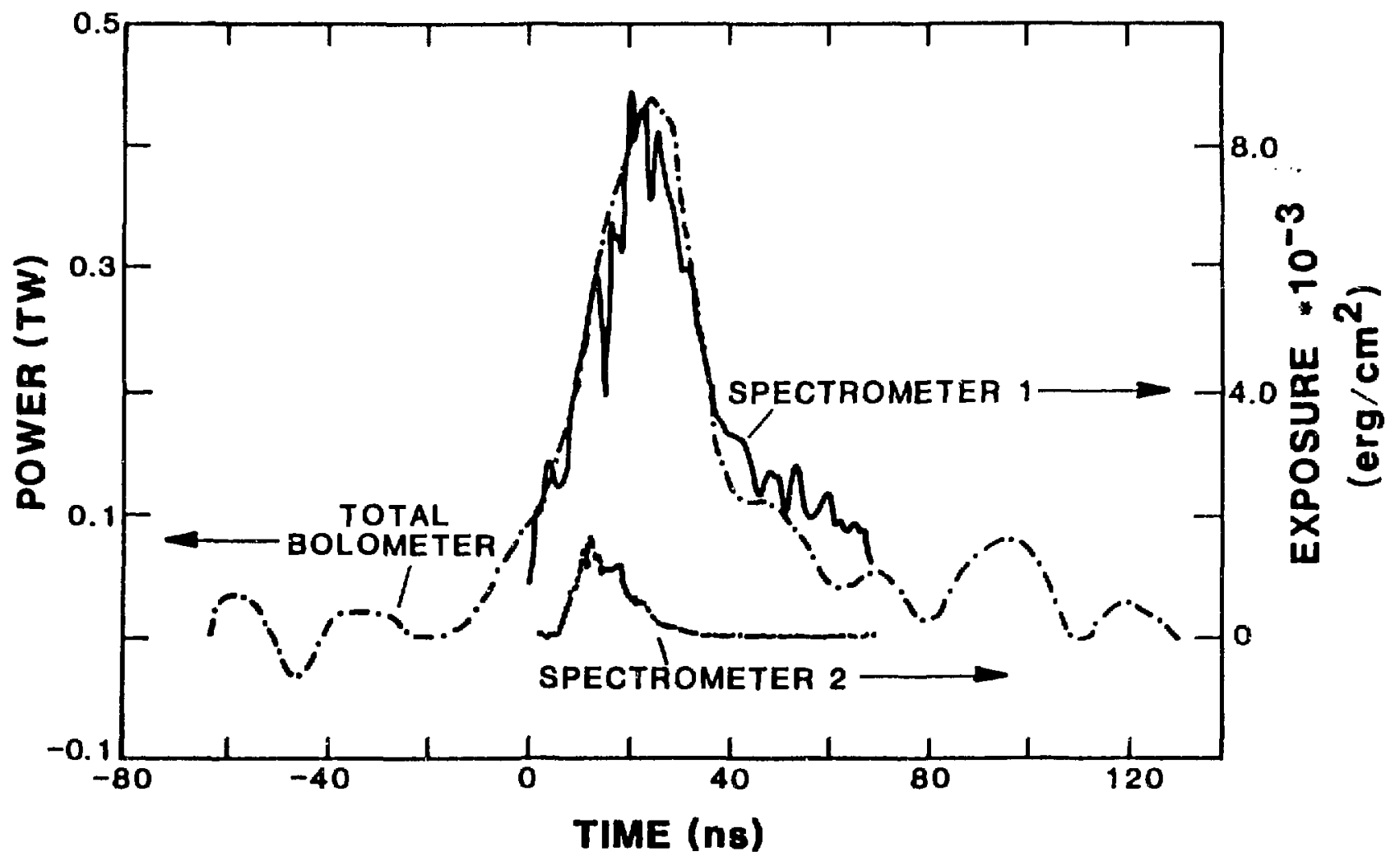

Figure 62. Total bolometer and multilayer spectrometer data for shot 1872. The left vertical axis is the total power in $4 \pi$ er determined by the bolometer ignal. The right vertical axis is the exposure on the streak camera film for the multilayer epectrometers. 


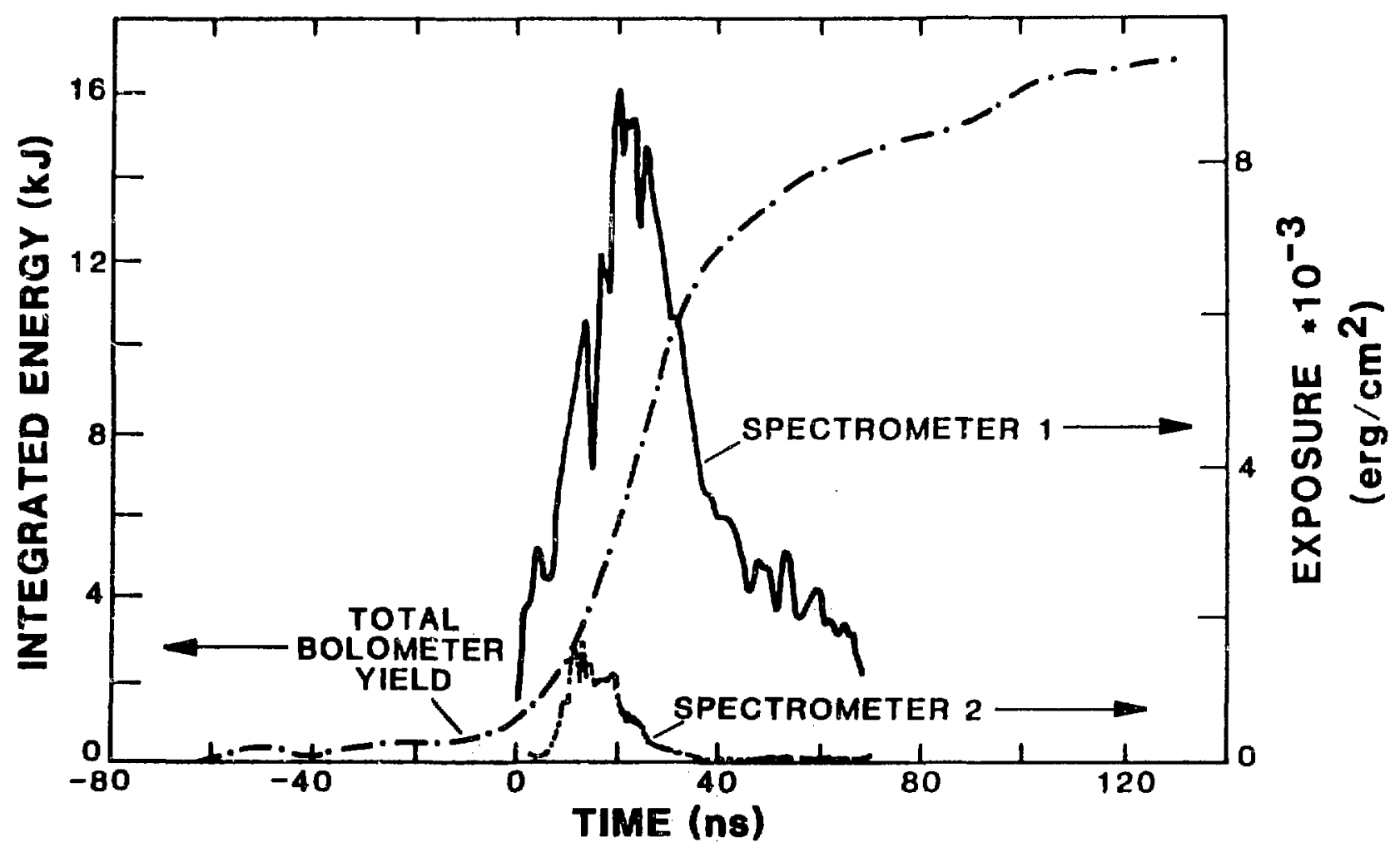

Elgure 63. The integrated total bolometer signa: and the data from the two multilayer spectrometers for shot 1872. The left vertical axis gives the total integrated energy. while the right vertical axis gives the exposure on the streak camera film for the two compressed streaks. 
channels existed, detailed modeling was attempted.

The Spectrum as a function of $t$ ime was deconvolved from the XRD data using the post-calibrated XRD responses and was shown in Figure 44. The difference botisen the total yields of the bolometers and XRD's was a factor of 9.4. However, as shown in Figure 48, the time history gives a scaling factor of 8.0 . The scaling factor of 8.0 was used to determine the upper limit of heating, and a scaling factor of 1 would then correspond to the XRD yield.

The multilayer parameters were initialized such that carbon was on top, the tungsten d-spacing was 24.98 \&. and the carbon layers had d-spacings of $42.52 \&$. The assumed initial densities were $19.3 \mathrm{~g} / \mathrm{cm}^{3}$ for the tungsten layers and $2.0 \mathrm{~g} / \mathrm{cm}^{3}$ for the carbon layers. The incidence angle was taken to be 6.25 degrees. The calculations were all done assuming unpolarized $x$ rays.

With the assumption of no conduction the multilayer code calculated that the first tungsten layer (second layer in the stack) would be melted by the end of the $x$ ray pulse, for the multilayer in spectrometer 1 at 150 em from the source. As post-shot $x-r a y$ measurements showed, this multilayer was not destroyed, that is, melted, substantiating what was known all along, that 15 , that this model was unrealistic. 
The calculations also showed that even up to near the melting temperature of the first tungsten layer, thermal expansion would not degrade the reflectivity over the spectral energy range of $25 \mathrm{eV}$ to $3.2 \mathrm{keV}$ more than 3 percent. However, thermal expansion would cause a shift of the Bragg diffraction peak as shown in Figure 64.

An average conduction factor can be determined by assuming that the melting of the first tungsten layer was the cause of the degradation in reflectivity. This assumption is not too unreasonable because at the melting of tungsten, carbon will diffuse into the tungsten very guickly. This can be seen in the following simplified discussion.

Fick's first law of diffusion (Askill, 1970) is given by

$$
J=-D_{-d c}^{d x} \frac{\text { atoms }}{c m^{2}-s e c}
$$

where $\underline{J}$ is the diffusion flux, $\underline{D}$ is the diffusion coefficient in units of $\mathrm{cm}^{2}$ /sec, and $\frac{d c}{d x}$ is the concentration gradient in atoms $/ \mathrm{cm}^{4}$. The temperaturedependent diffusion coefficlent can be expressed as $D=D_{0} \operatorname{sxp}(-Q / R T) \quad$. Here" $D_{0}$ is the frequency factor in $\mathrm{cm}^{2} / \mathrm{sec}$ (Askill, 1970), $Q$ is the activation energy in cal/mole, $\underline{R}$ is the 


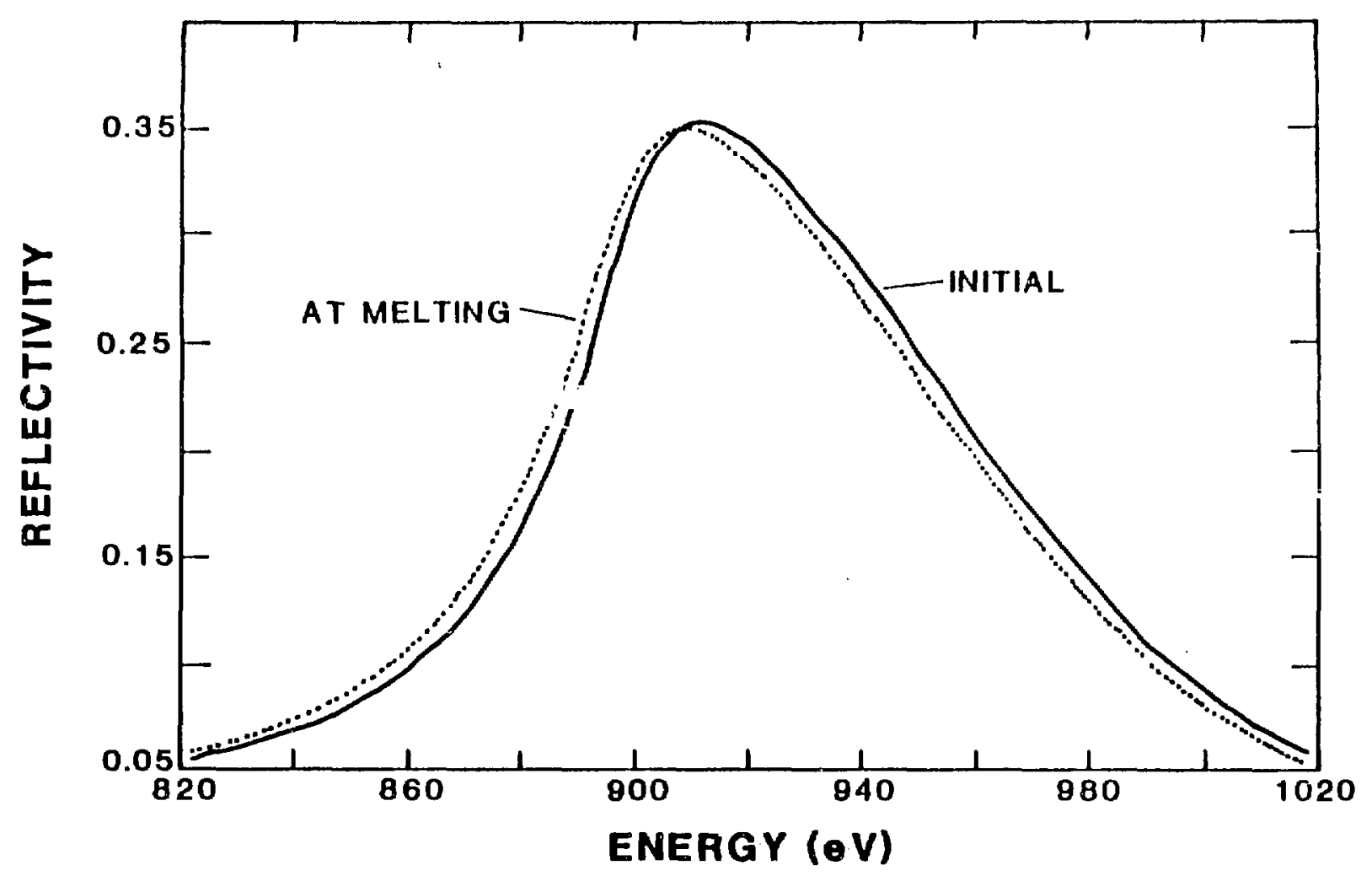

Figure 64. Reflectivity curve around the Bragg diffraction peak for the lnitial multilayer parameters and for the expanded multilayer parameters at the melting temperature of the flrst tungsten layer for shot 1872 . 
universal gas constant, and $T$ is temperature in degrees kelvin. For carbon diffusing into tungsten, $D_{0}$ is $8.9 \mathrm{x}$ $10^{-3} \mathrm{~cm}^{2} / \mathrm{sec}$, and its activation energy is $53.5 \mathrm{kcal} / \mathrm{mole}$ in the temperature range of $1300-19000^{\circ}$ (Askill, 1970). At higher temperatures these numbers could be sightly larger: however, these are adequate for the rough estimates. If the concentration of the carbon is taken to be that of the pure carbon layer and zero at the center of the tungsten layer ( $\underline{\mathrm{dx}}=12.5 \AA$ ), there is a concentration gradient of dc $\frac{d c}{d x}=8.02 \times 10^{29}$ atoms $/ \mathrm{cm}^{4}$, assuming the density of the carbon layer is $2.0 \mathrm{~g} / \mathrm{cm}^{3}$. At the tungsten melting temperature, $3680^{\circ} \mathrm{K}$ the diffusion coefficient is given by

$D=0.76 \times 10^{-6} \frac{\mathrm{cm}^{2}}{\mathrm{sec}}$.

Multiplying the concentration by the diffusion coefficient gives

$J=4.62 \times 10^{24} \frac{\text { atoms }}{\mathrm{cm}^{2}-\mathrm{sec}}$.

The time it takes for one-half of a surrounding carbon layer ( $21.5 \mathrm{\&}$ ) to diffuse into the tungsten layer is $t_{d}$. This is determined by dividing the number of atoms per unit area in the half-carbun layer by the diffusion $f l u x$. In this case $t_{d}$ is $2.13 \times 10^{16}$ atoms $/ \mathrm{cm}^{2}$. The diffusion $t$ ime is therefore 
$t_{D}=\frac{N}{J}=\frac{2.13 \times 10^{16} \text { atoms } / \mathrm{cm}^{2}}{4.62 \times 10^{24} \text { atoms } / \mathrm{cm}^{2}-5 e c}=4.6 \mathrm{~ns}$.

This gives a rough time for the destruction of the interfaces at the melting temperature of tungsten, assuming that the tungsten layer is still solid. If the tungsten layer turns liquid, diffusion may be very much faster. In addition, there may be other processes that occur when the tungsten layer melts that degrade the reflectivicy very quickly.

Both Shot 1863 and Shot $\$ 1872$ exibited a small decay, then a "flat" signal level before a rapid decay began. For shot $\$ 1872$ the noise is such that this is hard to see but the dip is evident. By, assuming that melting of the first tungsten layer began at the beginning of the "flat" signal, the temperature profiles of the first and second tungsten layers would look like that shown in Figure 65 superimposed on the signal of Spectrometer 2. Since the spectral power is increasing during this time, the reflectivity is really decreasing. The transition time from solid to liquid is of the same order as the "flat" signal and also the diffusion time calculated above. After the "flat" signal the reflectivity then decays rapidly as the temperature of the first tungsten layer rises rapidly, until a shorter "flat" signal is observed around $21 \mathrm{~ns}$. The melting of the 


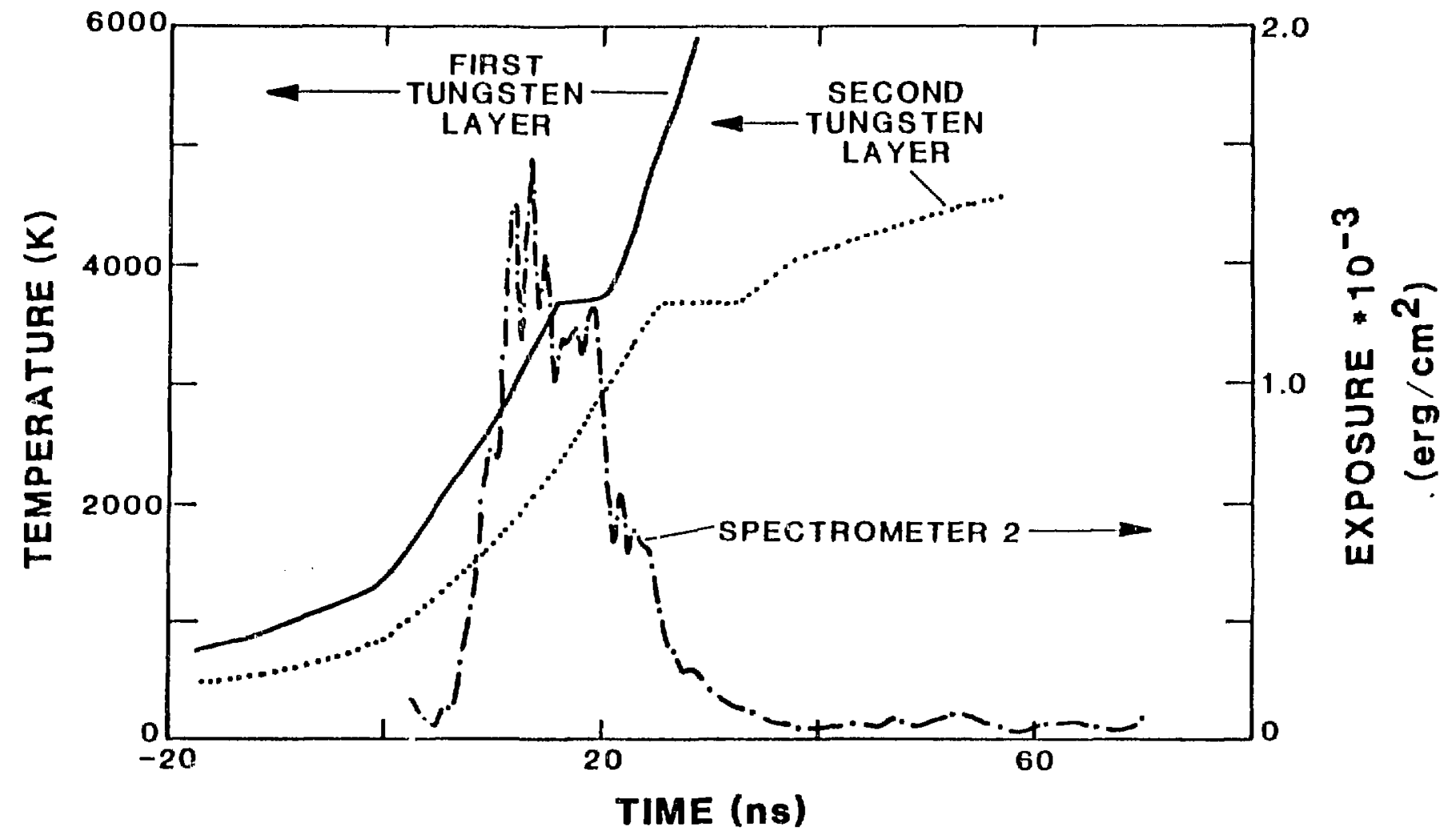

Figure 65. Multilayer Spectrometer 2 streak camera data and the calculated temperature profile as a function of $t$ ime for the two top tungsten layers. The right axis is exposire on the streak camera film for the two spectrometers. The left axis is the temperature of the layers. 
second tungsten layer occurs at the end of this shorter "flat" signal. It would be expected that the second layer would reach melting faster than indicated, because it would receive heat from the upper layers. The melting of the second tungsten layer may then possibly correspond to the second shorter "flat" signal.

In order to match the timing of the begirining of the first tungsten layer's melting with the beginning of the first "flat" signal, the XRD spectral data were multiplied by a factor of 0.112 . This indicates that conductivity reduced the heating of the multilayer by a factor of 10.2 for the low-yield limit. Using the more reliable bolometer yield would give an additional ractor of 8 , leading to a conductivity factor of 82 . That is, because of heat conduction, the multilayer can withstand 82 times as much heat input before the top tungsten layer reaches melting temperature as it would for zero conductivity.

\section{Discussion}

Both sets of data from Shot 1863 and 1872 imply that the observed degradation of the multilayer reflectivity was a multi-step process. Since the multilayer was reflecting a large specular component compared with that from Bragg diffraction, the destruc- 
tion of the multilayer reflectivity observed is indicative of a process occurring near the surface of the multilayer. The proposed mechanism of the carbon layers diffusing into the top tungsten layers and the melting of the tungsten layers is a surface proccess. It was calculated that differential thermal expansion of the multilayer could not explain the degradation seen. An average conductivity factor of 82 is not unreasonable.

These data indicate that tungsten-carbon multilayers are "two orders of magnitude more resilient to heat damage than "back of the envelope" calculations without conduction would indicate. 
The experiments of the flash $x$-ray loading of metal multilayers were presented in Part II. Chapter II-1 discussed the $x$-ray source eriteria and why PROTO II was chosen to be the $x$-ray source.

The time-integrated experiments were discussed and their results were presented in Chapter II-2. The timeintegrated experiments laid the foundation for the $t$ imeresolved experiments discussed in Chapter II-3. Timeresolved data of the degradation of the multilayer reflectivity were obtained; however, a large specular component and poor spatial zesolution prevented acquisition of distinct line profiles.

The methods used to determine the $x-r a y$ intensity emitted by the source, and thus incident on the multilayers, were discussed in Chapter II-4. The XRD gold photocathodes were post-calibrated and their measured responses were used to determine the spectral flux. Anisotropy factors were determined between the XRD data taken at 6.75 degrees from the z-axis, and bolometer data taken at 75 degrees from the z-axis.

Two different methods for calculating the absorptance of a layer in a multilayer were given in Chapter II-5. The first method was to calculate the difference 
in the Poynting vectors at different sides of the multilayer, and the second was calculating the absorptance by assuming the multilayer was an absorber. The heating model presented in Chapter II-5 ignored conduction but included thermal expansion.

Time-resolved data from two shots were analyzed in Chapter II-6. For Shot \#1863 two pieces of a tungstencarbon multilayer with a 2d-spacing of 135 \& were used at 6.3 degrees. The Spectrometer 2 multilayer at $15 \mathrm{~cm}$ recelved a total dose of $15 \mathrm{~J} / \mathrm{cm}^{2}$. The reflectivity of the multilayer of Spectrometer 2 decayed rapidy 28 ns from the beginning of the Spectrometer l streak camera trace. The corresponding power level at the multilayer was $420 \mathrm{Mw} / \mathrm{cm}^{2}$, and the integrated dose was $5.27 \mathrm{~J} / \mathrm{cm}^{2}$, as determined from the bolometer data.

For Shot \#1872 two pieces of a tungsten-carbon multilayer with a $2 \mathrm{~d}$ of 1358 set at 6.25 degrees were used. The reflectivity of the Spectrometer 2 multilayer at $15 \mathrm{~cm}$ decayed rapidly at $19.5 \mathrm{~ns}$ after the start of the streak trace of spectrometer 1. The corresponding power level and integratad dose determined from the bolometsr data were $141 \mathrm{Mw} / \mathrm{cm}^{2}$ and $2 \mathrm{~J} / \mathrm{cm}^{2}$, respectively. For Shot 1872 detailed modeling was attempted. It was calculated that thermal expansion alone could not explain the degradation of the multilayer reflectivity 
observed with Spectrometer 2. By matching the beginning of the first "flat" part of the Elgnal observed by Spectrometer 2 ws: $=$ the start of melting of the first tungsten iayer (second layer in the multilayer stack), conducivity factors of 10,2 and 82 for the amount of heat that could be absorbed before melting were determined from upper and lower estimates of the spectral flux. Since the bolometer data were taken at the same angle from the z-axis as the multilayer data, the factor of 82 was felt to be more reliable than the factor of 10.2 from XRD Epectral data taken at an angle where obscuration of the line of sight could occur. This conduetivity factor indicates that tungsten-carbon multilayers are nearly two orders of magnitude more resilient to heat damage than "back of the envelope" calculations without conduction would indicate. 
FUTURE WORK

Because of the small incidence angles used in this investigation, the specular component reflecting from the multilayers and into the time-resolved detector system was so large that it washed out the line information, and thus the detalled information of the $\underline{R}$ versus $\theta$ curve that was desired. Such information, if obtained, would have been complicated by the finite and large size of the plasma source as seen by the multilayer $15 \mathrm{~cm}$ away. This experiment should be performed, if possisle, with a smaller size Eource, and with larger incidence angles and better filtration to remove the specular component. This would give valuable information on the Bragg diffraction peaks with mininmal background.

A further difficulty which was encountered that could be avoided was the determination of the $x-r a y$ spectral flux. Ideally one would like an infinite number of XRD's with narrow bandpasses over the spectral region of interest. If a similar experiment were to be run, it would be important to have the diagnostice that are to guantify the spectrum to be at the same angle from the plasma axis as the multilayers. This would eliminate the problem of anisotropy in the $\underline{\theta}$ direction.

The fluorescer-fiber optjc-streak camera system 
could be made both more sensitive and to have better spatial and temporal resolution. It would help to shorten the fiber bunde by bringing the streak camera closer to the machine in its own EMP-shielded box. The fiber and the fluorescer could be changed such that the wavelength at which the fluorescer emits is more suited to being passed by the fiber optic.

If the improvements in the experimental design outlined above were done, information on how the multilayer's Bragg diffraction is degraded covid be determined. It would also be valuable to investigate how the multilayer diffraction properties would change as the time scale of the $x-r a y$ heat loading was varied. This information would have direct application to the design and implementation of multilayer $x$-ray laser cavity mirrors, synchrotron monochromators, and multilayers used as high-temperature plasma diagnostics. 


\section{LIST OF REFERENCES}

Askill, J., Tracer Diffusion Data for Metals, Alloys, and Simple Oxides (IFI/Plenum, New York, 1970).

Auerbach, J. M. and K. G. Tirsell, "Software for Reflectivity Calculations of X-Ray Mirrors," Lawrence Livermore National Laboratory Report UCRL$91330(1985)$.

Barbee, T. W.. Jr.. "Sputtered Layered Synthetic Microstructures (LSM) Dispersion Elements," in AIP Con ference Proceedings $\# 75$ on Low Energy X-Ray Diag nostics-1981, edited by D. T. Attwood and B. $L$. Henke (American Institute of Physics. New York, $1981)$. p. 131 .

and D. C. Keith, "Synthetic Structures Layered on the Atomic Scale," in Workshop on X-Ray Instrumentation for Synchrotron Radiation Research. edited by H. Winick and G. Brown. Stanford Synchrotron Radiation Laboratory Report SSRL No. 78/04 (1978).

and J." H. Underwood, "Solid Fabry-Pero: Etalons for X-Rays," Opt. Comm. 48, 161 (1983).

Bartlett, R. J., W. J. Trela, D. R. Kania, T. W. Barbee, M. P. Hockaday, and $P$. Lee, "Soft X-Ray Measurements of Solid Faury-Perot Etalons," Opt. Comm. 55, 229 (1985).

Beckmann, P., and A. Spizzichino, The Scattering of Electro-magnetic Waves from Rough Surfaces (Pergamon, New York, 1963).

Bennent, H. E. and J. M. Bennent, "Precision Measurements in Thin Film Optics," Phys. of Thin Films 4 , 1 (1969).

Berning, P. H., "Theory and Calculations of Opticai Thin Films," Phys. Thin Films 1. 69 (1963).

Born, M., and E. Wolf, Princlples of Optics, 6th ed. (Pergamon, New York, 1983) Sec I.6.

Carniglia, C. X. "Scalar Scattering Theory for Multilayer Optical Coatings," Opt. Eng. 18, 104 (1979). 
Ceglio,- N. M., D. G. Stearns, and A. M. Hawryluk, "Multilayer Structures for $X-R a y$ Laser Cavities," in Applications of Thin-Film Multilayered Structures to Fiqured X-Ray Optics (SPIE, Bellingham, Washington, 1985), Vol. 563, p. 360 .

Compton, A. H., and S. K. Allison, X-Raye in Theory and Experiment, 2nd ed.. (Van Norstrand, New York, 1935).

Day, R. H., and T. Barbee, "Application of Layered Synthetic Microstructures to High Temperature Plasma Diagnostics," Rev. Sci. Instrum. 56, 791 (1985).

P. Lee, E. B, Saloman, and Diodes for Laser Fusion Plasma

D. J. Nagel, "X-Ray ( $1981 a)$. Alamos Scientific Laboratory Report LA-7941-MS

Diagnostics," LOS

\section{" C_. and "Photoelectric Quantum Efficiencies and Filter Hindow Absorption Coefficients from $20 \mathrm{eV}$ to $10 \mathrm{keV}, " \mathrm{~J}$. Appl. Phys. 52. 6965 ( $198 \mathrm{lb})$.}

Deubner, .. "Versuche uber die Reflexion von Rontgenstrahlen an einem kunstlich hergesteliten Schichtenkorper," Ann. d. Phys. 5,261 (1930).

Dinklage, J., "X-Ray Diffraction by Multilayered ThinFilm Structurss and Their Diffusion," J. Appl. Phys. 38, $3781(1967)$.

DuMond, J., and J. P. Youtz, "Selective X-Ray Diffraction from Artificially Stratified Metal Filiws Deposited by Evaporation," Phys. Rev. 48, 703 (1935). - and "An X-Ray Hethod for Determining Rates of Diffusion in the Solid State," J. Appl. Phys. 11, 357 (1940).

Eastman. J. M. " "Scattering by All-Dielectric Multilayer Bandpase Filters and Mirrors for Lasers," Phys. of Thin Films 10, 167 (1978).

Edwards, D. F., "Silicon (Si)," in Handbook of Optical Constants of Solids, edited by E. D. Palik, (Academic Press, New York, 1985), p. 553. 
EG\&G Santa Barbara, "Project Summary LANL Support Program January-March 1981," EG\&G Energy Measurements Group. Santa Barbara, California, report EGG 1183-2427 (1981).

Elson, J. M., J. P. Rehn, and J. M. Bennent, "Relationship of the Total Integrated Scattering from Hultilayer-Coated Optics to Angle of Incidence, Polarization, Correlation Length, and Roughness Cross-Correlation Properties." Appl. Opt. 22. 1307 (1983).

Galileo Electro-Optics Corp.. "Data Sheet 1000, Fiber Optic Faceplates" (Sturbridge, Massachusetts, 1981).

Gilfrich, J. V., D. J. Nagel, N. G. Loter, and T. Barbee. "X-Ray Characteristics and Applications of Layered Synthetic Microstructures," in Advances in X-Ray Analysis, edited by Russ, Barrett, Predicki, and Leyden (Plenum Press, New York, 1982), Vol. 25. p. 255 .

Haelbich, R. P., C. Kunz, and E. Spiller, "Multilayer Coatings foz Soft $X$-Rays status Report (March 1978) of Work," in horkshop on X-Rey Instrumentation for Synchrotron Radiation Research, edited by H. Winick and G. Brown, SSRL No. $78 / 04$ (1978) p. VI-50.

Hagemann, H. J.. W. Gudat, and C. Kunz, "Optical Constants from the Far Infrared to the X-Ray Region: $\mathrm{Mg}$, Al, Cu, Ag, Au, Bi, C, and Al203," Deutscbes Elektronen-Synchrotron report DESY SR-74/7 (1974).

Hammel, B. A., and L. A. Jones, "Effects of Internally Produced Nonthermal Electrons on the Temperature Diagnostics of a Hollow Gas shell Z-Pinch," Appl. Phys. Lett. 44, 667 (1984).

Handbook of Chemistry and Physics, 6oth ed., (The Chemical Rubber Co.. Cleveland, Ohio, 1980), p. E-47.

Hanson, D. L., "Bolometer Data," in Proqress Report: Narya Pulsed-Power-Dr Iven X-Ray Laser Proqram January 1983-December 1983, edited by M. K. Matzen and R. B. Spielman, Sandia National Laboratories. Al buquerque. New Mexico, report SAND-84-1587 ( 1984 ). p. 29 .

Henke. B. L., "Low Energy X-Ray Spectroscopy with Crystals and Multilayers," in AIP Conference Proceedings $\$ 75$ on Low Energy X-Ray Diaqnostics-1981, 
edited by D. T. Attwood and B. L. Henke (American Institute of Physics. New York, 198la), p. 85.

"Low Energy X-Ray Interactions: Photoionization. Scattering. Specular and Bragg Reflection," in AIP Conference Proceedings $\$ 75$ on Low Energy $x$-Ray Diaqnostics-1981, edited by $D$. T. Attwood and B. $L$. Henke (American Institute of Physics, New York, 198 1b), p. 146.

F. G. Fujiwara, M. A. Tester, C. H. Dittmore, and M. A. Palmer, "Low Energy X-Ray Response of Photographic Films: Part II. Experimental Characterization," J. Opt. Soc. Am. B 1,828 (1984b).

S. L. Kwok, J. Y. Uejio, H. T. Yamada, and G. C. Young, "Low Energy X-Ray Response of Photographic Films: Part 1. Mathematical Models." J. Opt. Soc. Am. B 1. 818 ( $1984 a)$.

and P. Lee, T. J. Tanaka, R. L. Shimabukuro, and B. K. Fujikawa, "Low Energy 8-Ray Interaction Coefficients: Photoabsorption, Scattering, and Reflection. $E=100-200$ eV $Z=1-94, "$ At. Data Nucl. Data Tables 27, 1 (1982). Yamada, "The Characterization of Multilayer Analyzers - Models and Measurements," in Applications of Thin-Film Multilayered Structures te Fiqured 8 -Ray Optics," edited by G. F. Marshall (SPIE, Bellingham, Washington, 1985), Vol, 563, p. 195 (1985).

Characterization of Multilayer X-Ray Analyzers: Hodels and Measurements," Opt. Eng. 25, 937 (1986).

Henry, J. P., E. Spiller, and H. Weisskopf, "Imaging Performance of a Normal Incidence Soft X-Ray Telescope," Appl. Phys. Lett. 40, 25 (1982).

Hodson. E. K., D. K. Thayer, and C. Frankl in, "Adapt Ive Gaussian Filtering and Local Freguency Estimates Usi.ng Local Curvature Analysis." in IFEE Transact ions on Acoustics, Speech, and Signal Processing, Vol. Assp-29, 854 (1981).

Hultgren, R., P. D. Desai, D. T. Hawkins, M. Gleiser, K. $K$. Kelley, and D. D. Wagman, "Carbon," Selected Values of the Thermodynamic Properties of the 
Elements (American Society of Metals. Metals Park, Ohio. 1973a)

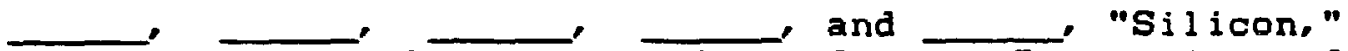
Selected Values of the Thermodynamic Properties of the Elements (American Society of Metals, Metals Park. Ohio 1973b).

Se lected Ualue of the Thermod and Selected Values of the Thermodynamic Properties of the Elements (American Society of Metals. Metals Park. Ohio, 1973c).

Johnson, D. L.. "Initial PROTO II Pulsed Power Tests", Sandia National Laboratories, Albuquerque, New Hexico, report SAND-76-5811 (1976).

Kohler, D. A., J. L. Guttman, B. A. Watson, and M. Gerassimenko. "Pulsed X-Ray Induced Damage to Metai and Hultilayer kirrors," Rev. Sci. Instrum. 56, 812 (1985).

J. G. Pronko, R. K. Bardin and L. F. Chase, "Time- and Energy-Dependent Effects in Crystal Spectrometers," Lockheed Palo Alto Laboratory report DNA-4774f (1978).

Lee, P., "X-kay Diffraction in Multilayers," Opt. Commun. 37. $159(1981)$.

"Uniform and Graded Mulilayers as X-Ray Optical Elements," Appl. Opt. 22, 1241 (1983).

Logan, J. D., R. S. Lee, R. C. We1ngart, and K. S. Yee, "Calculations of Heating and Burst Phenomena in Electrically Exploded Foils," J, Appl. Phys. 48, 621 (1977).

Lutz, S., private communication, August 18, 1986.

Lynch, D. W., and W. R. Hunter, "Optical Constants of Metals," in Handbook of Optical Constants of Sol1ds. E. D. Palik, ed. (Academic Press, NY, 1985), p. 359.

Lyons, P. B., R. H. Day, D. H. Lier, and T. L. Elsberry, "Sub-KeV X-Ray Calibration of Plastic Scintillators," ERDA Symposium on $X$ - and Gamma-Ray Sources and Applications, University of Michigan, Ann Arbor, Michigsa, 1976, Los Alamos Scientific Laboratory document LA-UR-76-189. 
and D. W. Lier, "Fabrication and X-Ray Calibration of Thin Plastic Scintillator Detectors," in IEEE Trans. Nucl. Sc1. NS-22, 88 (1975).

and J. Stevens, "Time Response of Plastic Scintillators," Nucl. Inst. Meth. 114, 313 (1974).

Martin, T. H., D. L. Johnson, and J. F. Seaman, "PROTO II Description," Sandia Nationel Laboratorles. Albuquerque, NM, report SAND 76-5964 (1976b).

Martin, T. H., J. P. Van Devende:, D. L. Jonnson, D. H. McDariel, and M. Aker, "PROTO II - A Short Pulse Hater Insulated Accelerator." in Proceedings of the First Intefnational Topical Conference on Electron Beam Research and Technology, Vol. 1, 450 (1976), also Sandia National Laboratories. Albuquerque, NM, report SAND 76-5122 (1976a).

Maxon, S., and T. Wainweight, "Radiation Spectra from an Imploding Argon Gas Puff," Phys. Fluids 27, 2535 $(1984)$.

Mix, L.P., E. J. T. Burns, D. L. Fehl, D. L. Hanson, and D. J. Johnesn, "Low Energy X-Ray Emission from Light Ion Targets," in AIP Conference Procesdings $\$ 75$ on Low Energy $X$-Ray Diagnostics-1981, edited by $D$. $T$. At twood and $B$. L. Henke (American Institute of Phys 1ce, New York, 1981), p. 25.

Nagel, D. J., T. w. Barbee, Jr. and J. V. Gilfrich, "Graded - Layer - Thickness Bragg X-Ray Reflectors," in Reflecting Optics for Synchrotron Radiation, edited by M. R. Howells (SPIE, Bellingham, Washington, 1981), Vol. 315, p. 110.

J. V. Gilfrich and T. W. Barbee Jr., "Bragg Diffractors with Craded - Thickness Multilayers," Nucl. Instrum. and Meth. 195, 63 (1982).

Ogle, J.. private communication, Jaruary 14, 1985.

Parratt, L. G., "Surface Studies of Sollds by Total Reflection of X-Rays," Phys. Rev. 95, 359 (1954).

Perkins, R. T., and L. V. Knight, "An Anayltic Solution of Darwin's Difference Equations," Acta. Cryst. A40. 617 (1984). 
Porteus. J. 0., "Relation Between the Height Distribution of a Rough Surface and the Reflectance at Normal Incidence,", J. Opt. Soc. Am. 53, 1394 (1964).

Pronko, J. G., T. R. Fisher, and L. F. Chase, "Scintillator Response Characteristics to Low Energy $X$ Rays." Lockheed Palo Alto Laboratory report DNA $4766 \mathrm{~F}$ ( 1978).

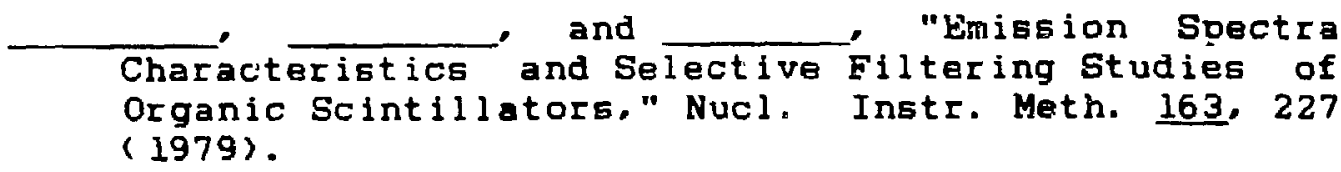

Rachocki, K. D., D. R. Brown, R. W. Springer, and P. N. Arendt, "Auger and Depth Profile Analysis of Synthetic Crystals for Dispersion of X-Rays," Appl. Surf. Sc1. 18, $165(1984)$.

Rosenbluth, A. E., and J. M. Forsyth, "The Reflecting Properties of Soft $X$-Ray Multilayers," in AIP Conference Proceedings $\$ 75$ on Low Energy 8 -Ray Diagnost1C8-1981, edited by D. T. Attwood and B. L. Herke (American Institute of Physics, New York, $1981)$, p. 280 .

Shirk, D. G., and N. M. Hoffman, "onte-Carlo Error Analys is in X-Ray Spectral Convolution," Rev. Sci. Instrum. 56, 809 (1985).

Spielman, R. B., D. L. Haneon, M. A. Palmer, M. K. Matzen, T. W. Hussey and J. M. Peek, "Efficlent Xray Production from Ultrafas - Gas-Puff Z Pinches," J. Appl. Phys. 57, $830(1985)$.

Spiller, E., "Multilayer Interference Coatings for the Vacuum Ultraviolet," in Space Optics, Pcoceedings of ICoIX, Santa Monica, 1972 (National Academy of Science, Washington, D.C., 1974), p. 581.

"Reflective Multilayer Coatings for the Far UV Region," Appl. Opt. 15, 2333 (1976).

A. Segmuller, J. Rife, and R. P. Haelbich, "Controlled Fabrication of Multilayer Soft $X$-Ray Mirrors," Appl. Phy. Lett. 37, 1048 (1980).

Takagi, Y., S. A. Flessa, K. L. Hart, D. A. Pawlik, A. M. Kadin, J. L. Wood, J. E. Keem, and J. E. Tyler, "Thermal Stability of w/C Multilayer Films," in 
Applications of Thin Film Multilayered Structures to Fiqured X-Ray Optics, (SPIE, Bellingham, Hashington, $1985), \underline{190} 66$.

Touloukian, Y. S., R. K. Kirby, R. E. Taylor, ana T. Y. R. Lee, Thermophysical Properties of Matter, TRPC Data Series, Vol. 13: Thermal Expansion, "Nonmetallic Solids"( IFI/Plenum, New York, 1975).

R. W. Powell, C. Y. Ho, and P. G. Kiemens, Thermophysical Properties of Matter, TRPC Data Seribs, Vol. 1: Thermal Conductivity. "Nonmetallic Elements and Alloys" (IFI/Plenum, New York, 1970a).

R. E. Taylor, and P. D. Desa1, Thermophysical Properties of Matter, TRPC Data Series, Vol. 12: Thermal Exansion, "Metallic Elsments and Alloys" (IFI/Plenum, New York, 1970b).

Underwood, J. H., T. w. Barbee, and D. C. Keith, "Layered Synthetic Microstructures: Properties and Applications in X-Ray Astronomy," in Space OpticsImaging $X$-Ray Optics Workshop, (SPIE, Bellingham, Washington, 1979), Vol. 184, p. 123.

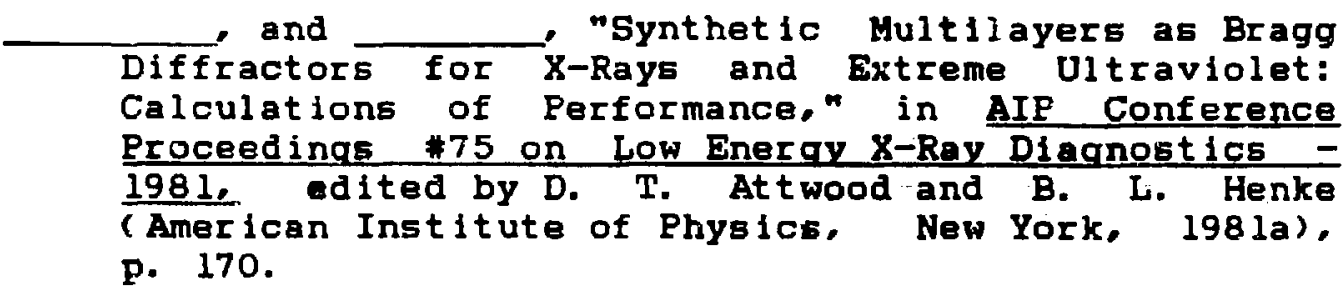

"Layered Synthetic Microstruc-
tures as Bragg Diffractors for X-Rays and Extreme
Ultraviolet: Theory and Predicted Performance,"
Appl. Opt. 20, 3027 ( $1981 \mathrm{~b})$.

Weaver, J. H., C. Krafka, D. W. Lynch, and E. E. Koch. "Optical Properties of Metalg I: The Transition Metalb, O. $1 \leq h \leq 500$ eV, Ti, V, Cr, Mn, Fe, Co, Ni, $\mathrm{Zr}$, Nb, Me, Ru, Rh, Pd, Hf, Ta, W, Re, Os, Ir, Pt," Deutsches Elektronen-Synchrotron report DESY F4l or HASYLAB 81/01 (1981).

Weinstein, w. "The Reflectivity and Transmissivity of Multiple Thin Coatings," J. Opt. Soc. Am. 37, 57b (1947). 
Zachariasen, W. H. , Theory of X-Ray Diffraction in Crystals (Wiley, New York, 1945). 


\section{AFPENDIX A}

MULTILAYERS AND COPFER $L_{\sigma}$ MEASUREMENTS

The multilayers were manufactured on three-inchdiameter silicon substrates and were cut in strips of 0.5-inch width for the heat loading experiments. The sectioning and labeling conventions followed are shown in Figure Al. Each disk had a name assigned by the manufacturer, and that label was kept for identification. The multilavers investisatej $\equiv$ ra their general characteristics are given in Table Al.

Each multilayer secticn was measured at $C_{L} L$. wavelength as described in Chapter I-1. The experimerta: results of these measurements are summarized in Table A2. 


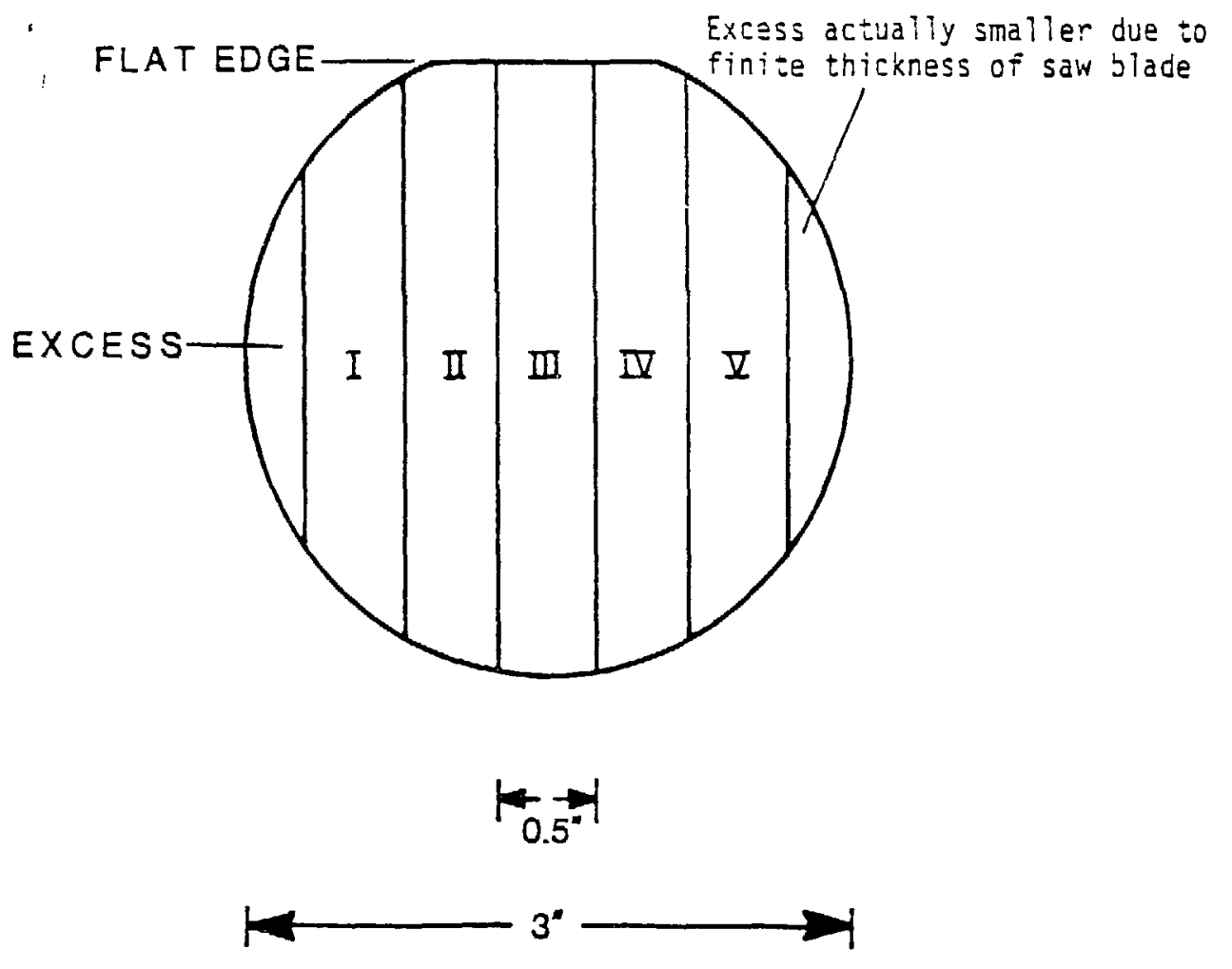

Figure Al. Sectioning and labeling used for three-inch multilayer disks. 
TABLE Al. MULTILAYERS INVESTIGATED.

\begin{tabular}{|c|c|c|c|c|}
\hline Name & Producer & Materials & $\begin{array}{c}\text { Reported } \\
2 \text { d-Spacing } \\
0 \\
\text { (A) }\end{array}$ & $\begin{array}{l}\text { Reforted } \\
\text { No. of } \\
\text { Layers }\end{array}$ \\
\hline \multicolumn{5}{|c|}{ a) } \\
\hline $83-021$ & Barbee & $\omega / C$ & 70 & 240 \\
\hline $83-0.23 \mathrm{~A}$ & Bártee & Mo/C & 140 & 120 \\
\hline $8:-023 B$ & Barbee & $\mathrm{Mo} / \mathrm{C}$ & 140 & 120 \\
\hline $83-132(3)$ & Barbee & $\mathrm{Mo} / \mathrm{C}$ & 72 & 220 \\
\hline $83-136(3)$ & Barbee & $P d / C$ & 136 & - \\
\hline \multicolumn{5}{|c|}{ b) } \\
\hline OVLA-070A-1 & Ovonic & $\mathrm{W} / \mathrm{Si}$ & 72 & 200 \\
\hline OVLA $-07 C A-2$ & Ovonic & $W / S i$ & 72 & 200 \\
\hline OVLA $-070 B-1$ & Ovonic & $\omega / C$ & 67 & 175 \\
\hline OVL.A-C:70B-2 & Dvonic & $\mathrm{W} / \mathrm{C}$ & 67 & 175 \\
\hline OVLA-13OB-1 & Ovonic & $\omega / C$ & 136 & 75 \\
\hline
\end{tabular}

a) T. W. Barbee, Jr., Stanford University, presently at Lawrence Livermore National Laboratory.

b)

Ovonic Synthetic Materials Company, Division of Energy Conversion Devices. Incorporated, Troy, Michigan. 
TABLE A2. MEASURED MULTILAYER REFLECTION PROPERTIES AT COPPER L WAVELENGTH.

\begin{tabular}{|c|c|c|c|c|}
\hline Section & $\begin{array}{l}\text { Percent } \\
\text { Reflection }\end{array}$ & $\begin{array}{l}\text { FWHM } \\
\text { (mrad) }\end{array}$ & $\begin{array}{l}\text { Integrated } \\
\text { Reflectivity } \\
\text { (mrad) }\end{array}$ & $\begin{array}{c}\text { Effective } \\
2 \text { d-spacing } \\
\text { o } \\
\text { (A) }\end{array}$ \\
\hline \multicolumn{5}{|l|}{$83-021$} \\
\hline I. & 21.0 & 6.8 & 1.7 & 71.3 \\
\hline II. & 22.0 & 6.5 & 1.7 & 71.9 \\
\hline III. & 22.0 & 6.3 & 1.7 & 71.9 \\
\hline IV. & 21.0 & 6.7 & 1.7 & 72.4 \\
\hline v. & 21.0 & 6.8 & 1.7 & 72.3 \\
\hline \multicolumn{5}{|l|}{$83-023 \mathrm{~A}$} \\
\hline I. & 4.1 & 5.0 & 0.25 & 117.3 \\
\hline II. & 4.8 & 4.0 & 0.24 & 115.9 \\
\hline III. & 4.6 & 4.0 & 0.23 & 115.3 \\
\hline IV. & 4.8 & 4.1 & 0.24 & 117.4 \\
\hline \multicolumn{5}{|l|}{$83-023 B$} \\
\hline I. & 5.3 & 4.3 & 2.8 & 118.4 \\
\hline II. & 4.0 & 4.2 & 2.3 & 118.9 \\
\hline III. & 4.6 & 4.0 & 2.3 & 119.6 \\
\hline IV. & 4.5 & 3.7 & 2.2 & 117.6 \\
\hline v. & 3.7 & 4.6 & 2.2 & 115.0 \\
\hline
\end{tabular}


PAELE R2. Continued.

\begin{tabular}{|c|c|c|c|c|}
\hline Section & $\begin{array}{l}\text { Percent } \\
\text { Reflection }\end{array}$ & $\begin{array}{l}\text { FWHM } \\
\text { (mrad) }\end{array}$ & $\begin{array}{l}\text { Integrated } \\
\text { Ref lectivity } \\
\text { (mrad) }\end{array}$ & $\begin{array}{c}\text { Effective } \\
2 \text { d-spacing } \\
0 \\
\text { (A) }\end{array}$ \\
\hline \multicolumn{5}{|c|}{$83-132(3)$} \\
\hline I. & 1.1 & 7.1 & 0.10 & 65.8 \\
\hline I I. & 2.7 & 4.4 & 0.14 & 66.5 \\
\hline III. & 2.8 & 4.0 & 0.14 & 67.4 \\
\hline IV. & 3.5 & 3.6 & 0.17 & 68.1 \\
\hline v. & 2.7 & 4.2 & 0.14 & 68.4 \\
\hline \multicolumn{5}{|c|}{$83-136(3)$} \\
\hline I. & 6.4 & 7.8 & 0.59 & 71.3 \\
\hline I I. & 6.3 & 7.7 & 0.58 & 71.8 \\
\hline III. & 6.4 & 7.9 & 0.62 & 71.6 \\
\hline IV. & 6.5 & 7.7 & 0.61 & 71.5 \\
\hline v. & 6.5 & 7.9 & 0.61 & 72.1 \\
\hline \multicolumn{5}{|c|}{ OVLA-070A-1 } \\
\hline I. & 22.0 & 5.5 & 1.2 & 72.4 \\
\hline I I. & 23.0 & 4.9 & 1.1 & 70.0 \\
\hline I I I. & 27.0 & 4.2 & 1.5 & 68.6 \\
\hline IV. & 25.0 & 4.2 & 0.9 & 69.1 \\
\hline v. & 25.0 & 4.5 & 1.1 & 70.4 \\
\hline
\end{tabular}


TABLE A2. CONTINUED

\begin{tabular}{|c|c|c|c|c|}
\hline Section & $\begin{array}{l}\text { Percent } \\
\text { Reflection }\end{array}$ & $\begin{array}{l}\text { FWHM } \\
\text { (mrad) }\end{array}$ & $\begin{array}{l}\text { Integrated } \\
\text { Ref lectivity } \\
\text { (mrad) }\end{array}$ & $\begin{array}{c}\text { Effective } \\
2 \text { d-spacing } \\
0 \\
\text { (A) }\end{array}$ \\
\hline \multicolumn{5}{|c|}{ OVLA-070A-2 } \\
\hline I. & 22.0 & 5.2 & 1.4 & 73.1 \\
\hline II. & 23.0 & 5.0 & 1.4 & 71.1 \\
\hline III. & 26.0 & 4.3 & 1.4 & 69.1 \\
\hline IV. & 26.0 & 4.3 & 1.4 & 69.5 \\
\hline V. & 26.0 & 4.6 & 1.5 & 70.9 \\
\hline \multicolumn{5}{|c|}{ OVLA $-070 B-1$} \\
\hline I. & 11.0 & $E .5$ & 0.37 & 66.1 \\
\hline II. & 15.0 & 5.3 & 1.1 & 68.6 \\
\hline III. & 16.0 & 5.2 & 1.1 & 69.6 \\
\hline IV. & 16.0 & 5.3 & 1.1 & 69.5 \\
\hline v. & 14.0 & 5.8 & 1.0 & 68.5 \\
\hline \multicolumn{5}{|c|}{ OVLA-07OB-2 } \\
\hline I. & 13.0 & 6.9 & 1.0 & 64.6 \\
\hline II. & 16.0 & 5.6 & 1.1 & 66.6 \\
\hline III. & 17.0 & 5.2 & 1.2 & 67.8 \\
\hline IV. & 17.0 & 5.2 & 1.1 & 68.2 \\
\hline$\nabla$. & 15.0 & 5.6 & 1.1 & 67.3 \\
\hline
\end{tabular}


TrBLE A2, CONTINUED

\begin{tabular}{|c|c|c|c|c|}
\hline Section & $\begin{array}{l}\text { Percent } \\
\text { Reflection }\end{array}$ & $\begin{array}{l}\text { FWHM } \\
\text { (mrad) }\end{array}$ & $\begin{array}{l}\text { Integrated } \\
\text { Reflectivity } \\
\quad \text { (mrad) }\end{array}$ & $\begin{array}{c}\text { Effective } \\
2 \text { d-spacing } \\
0 \\
\text { (A) }\end{array}$ \\
\hline \multicolumn{5}{|c|}{$0 V I A-130 B-1$} \\
\hline I. & 25.0 & 8.2 & 2.3 & 107.6 \\
\hline II. & 22.0 & 8.5 & 2.1 & 110.9 \\
\hline III. & 25.0 & 8.3 & 2.3 & 112.0 \\
\hline IV. & 26.0 & 8.7 & 2.2 & 112.2 \\
\hline v. & 26.0 & 8.6 & 2.2 & 110.2 \\
\hline
\end{tabular}




\section{APPENDIX B. MULTILAYER COMPUTER CODE}

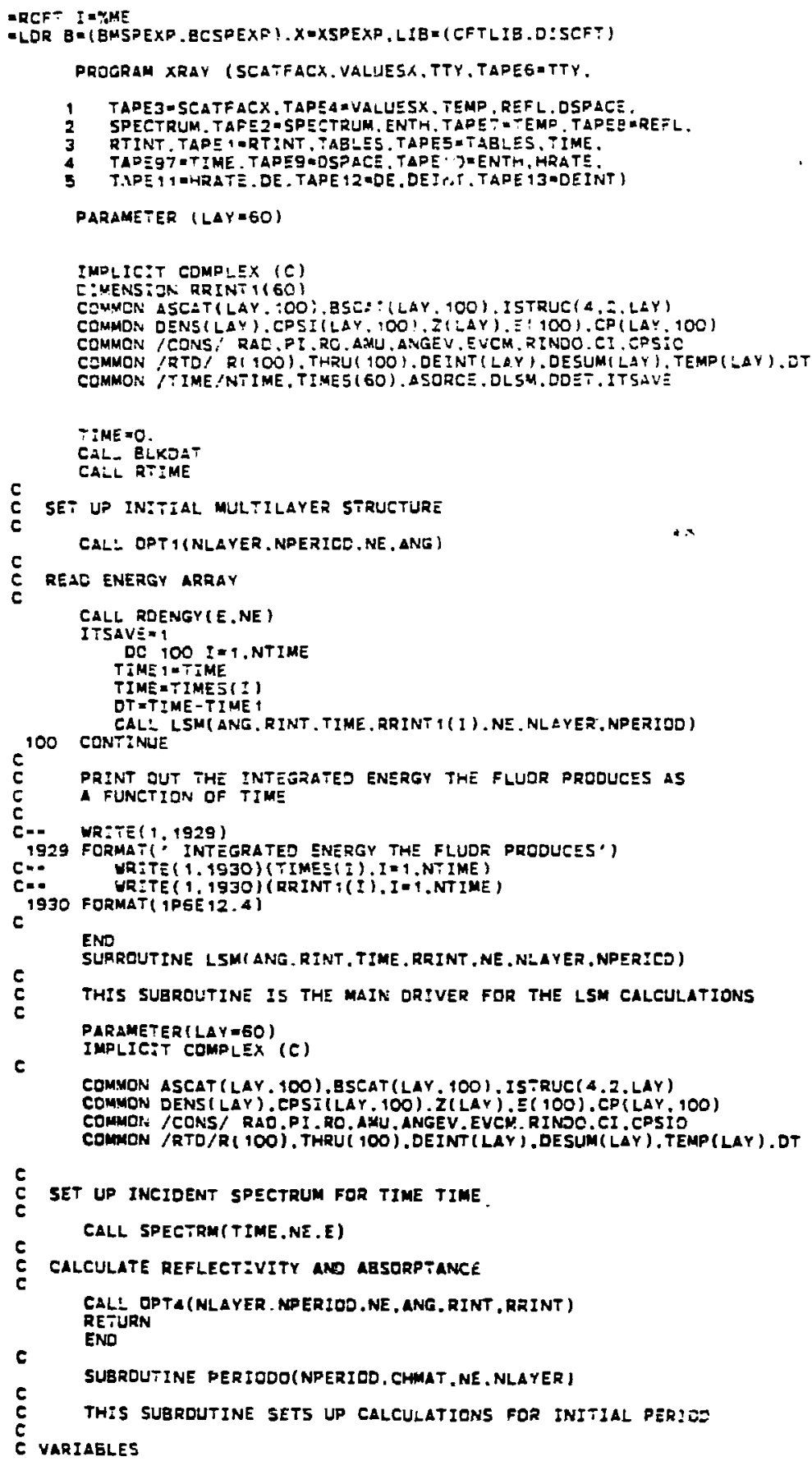




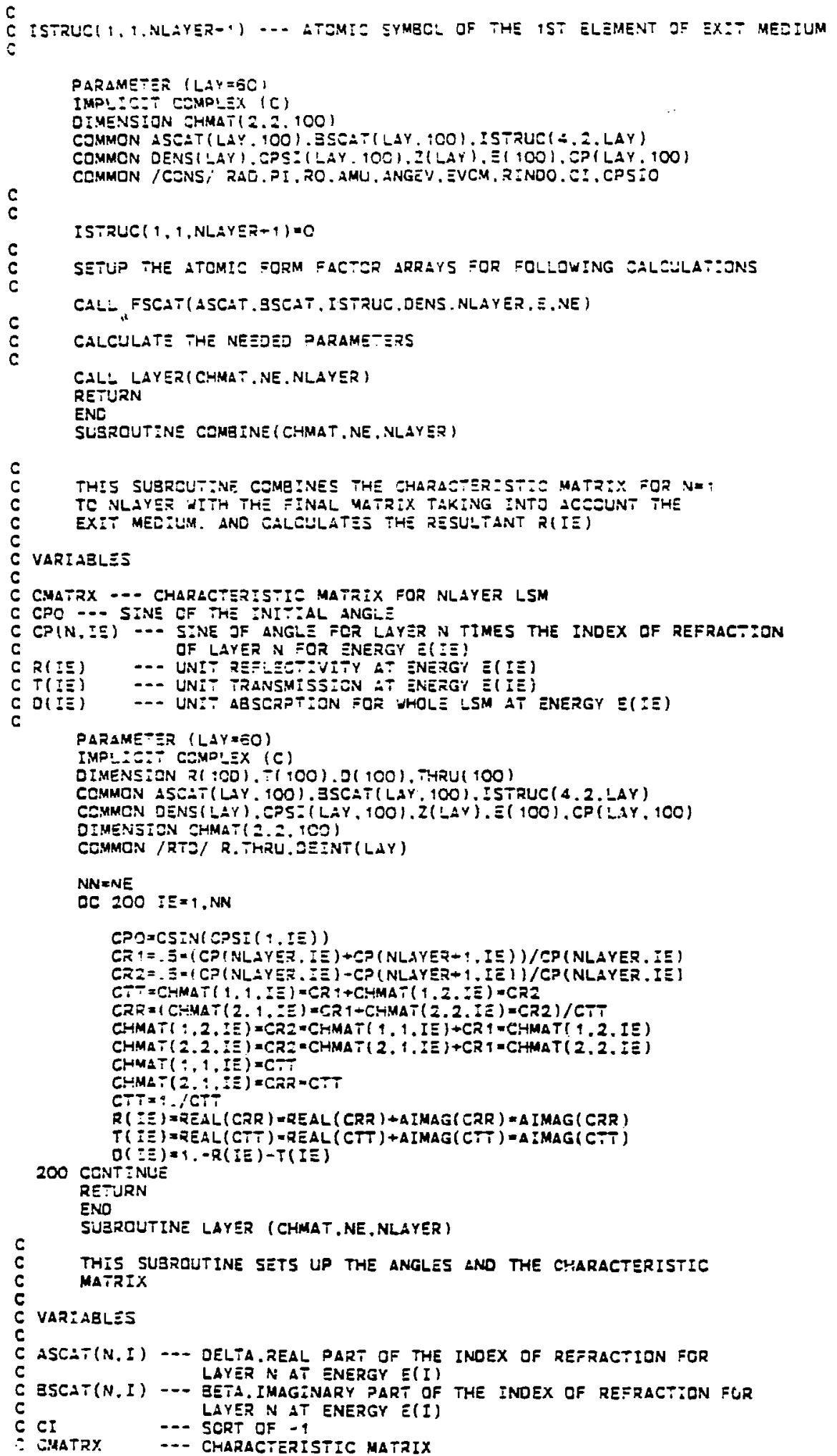




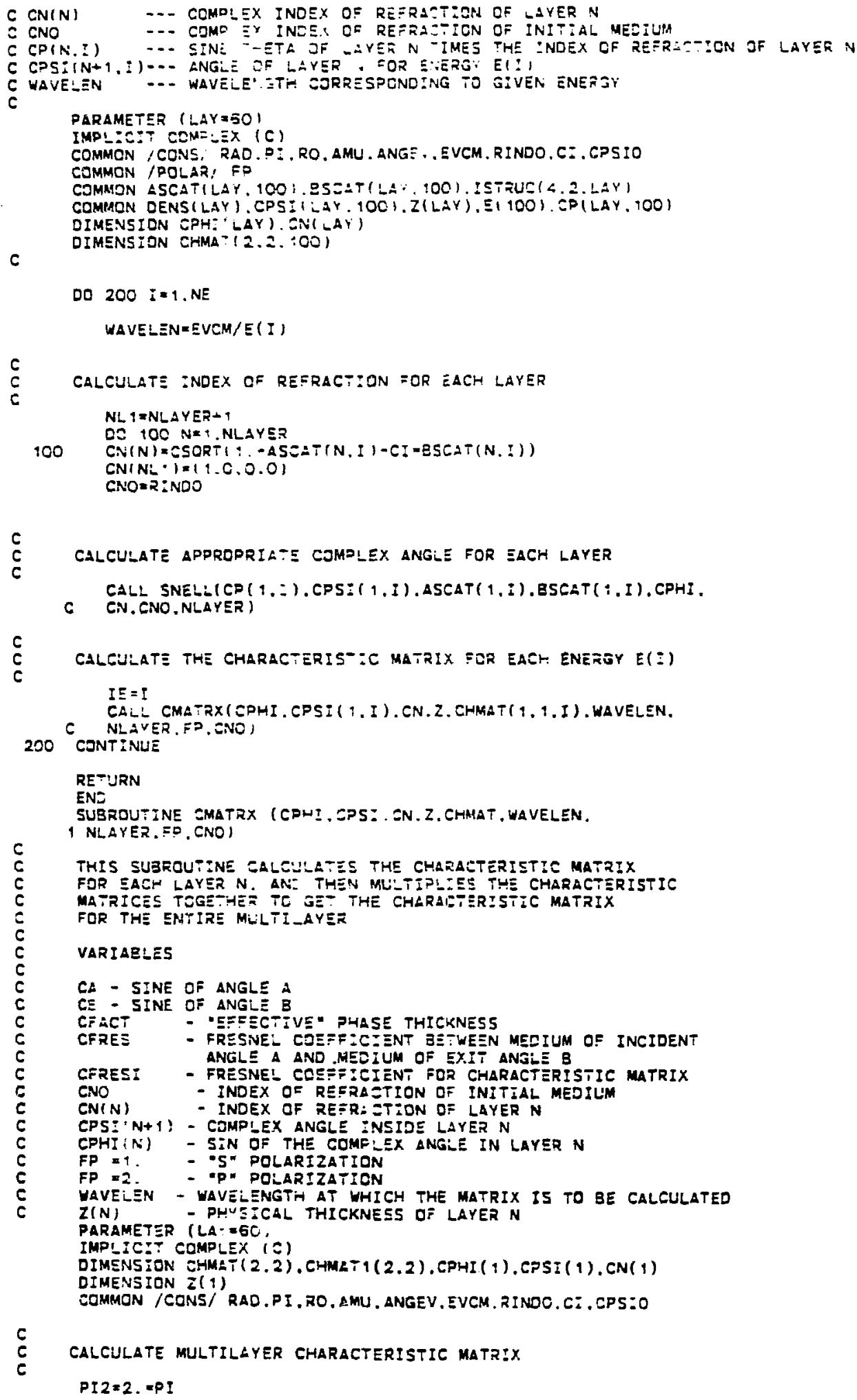




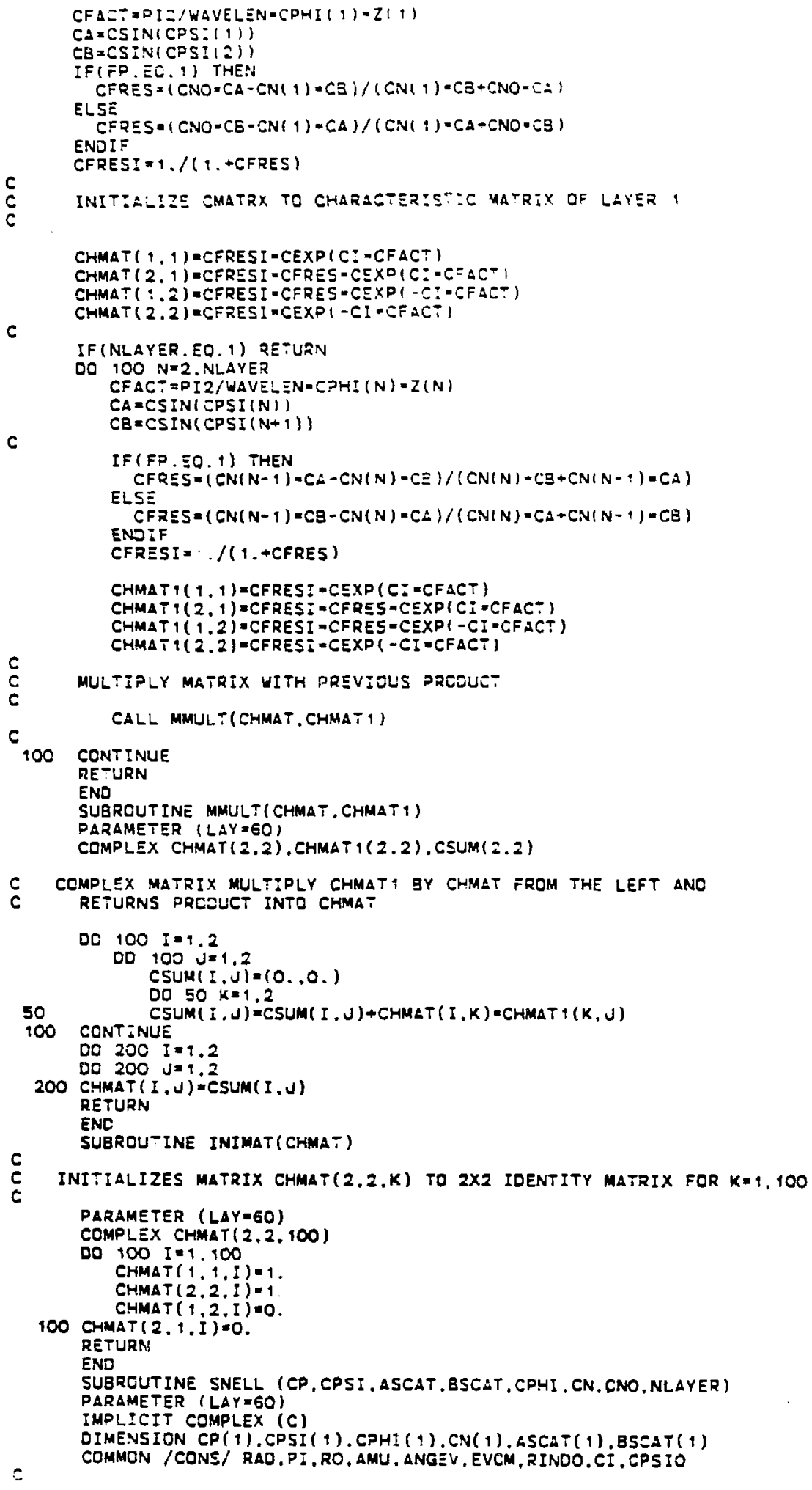




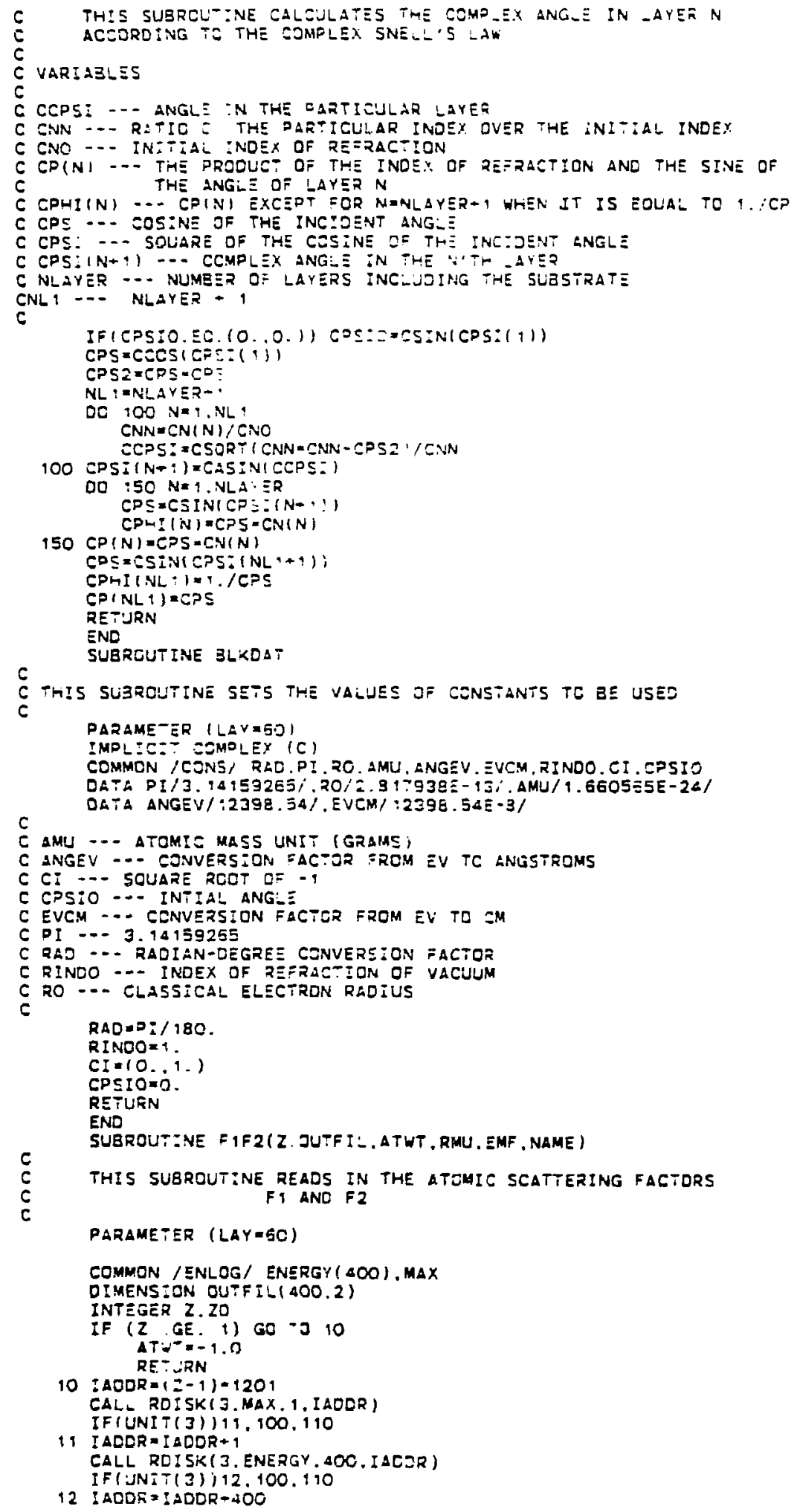




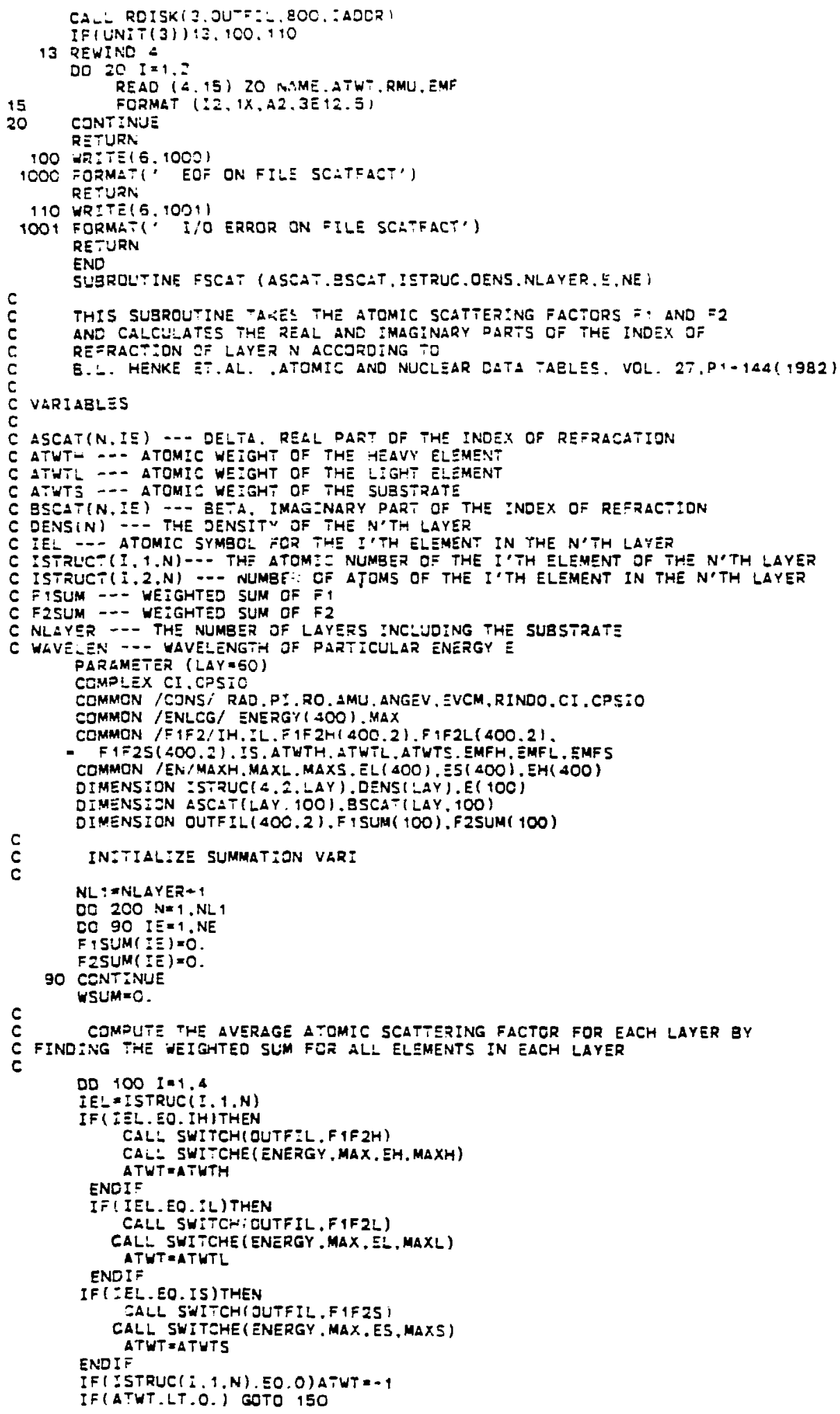




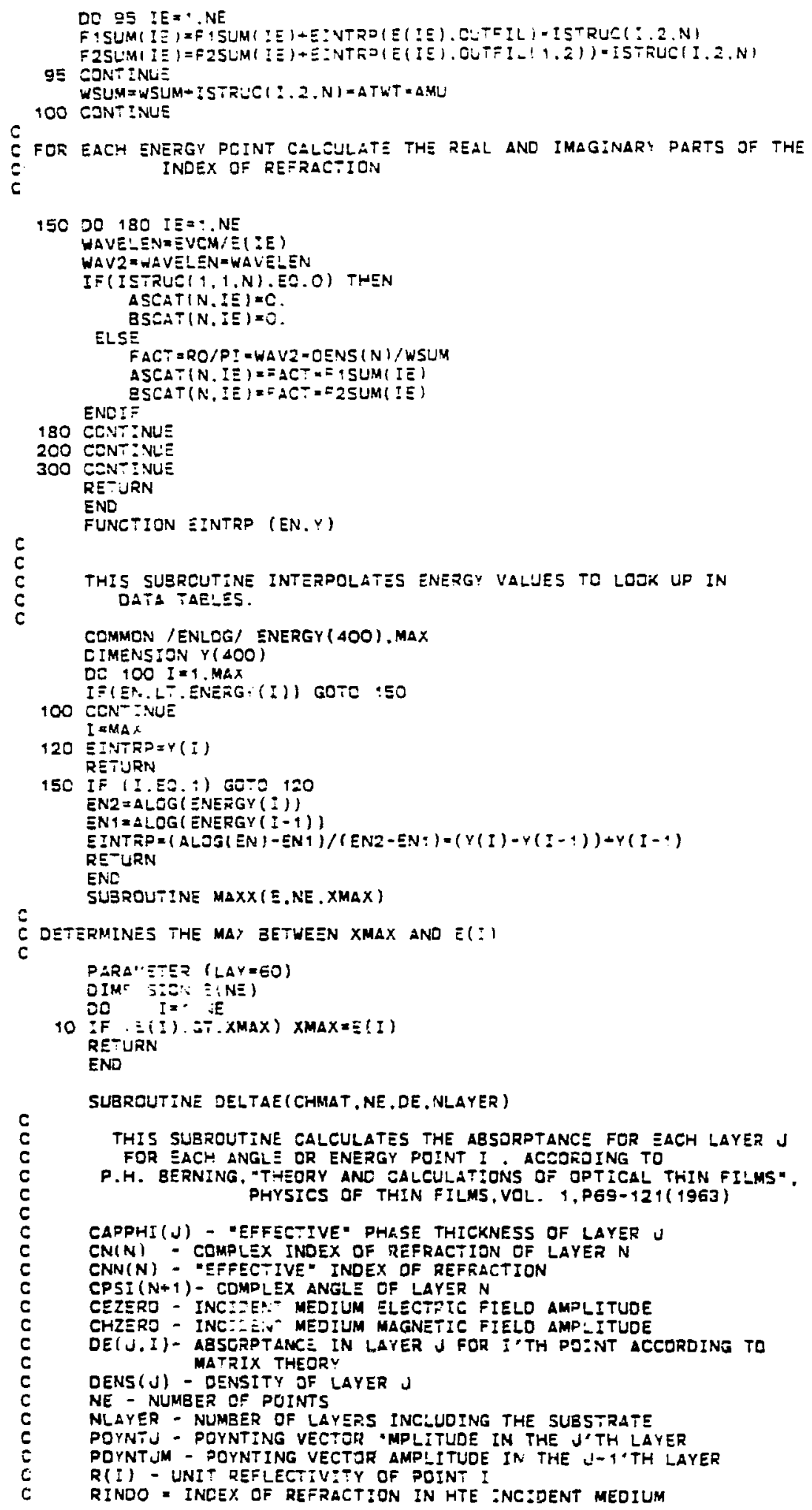




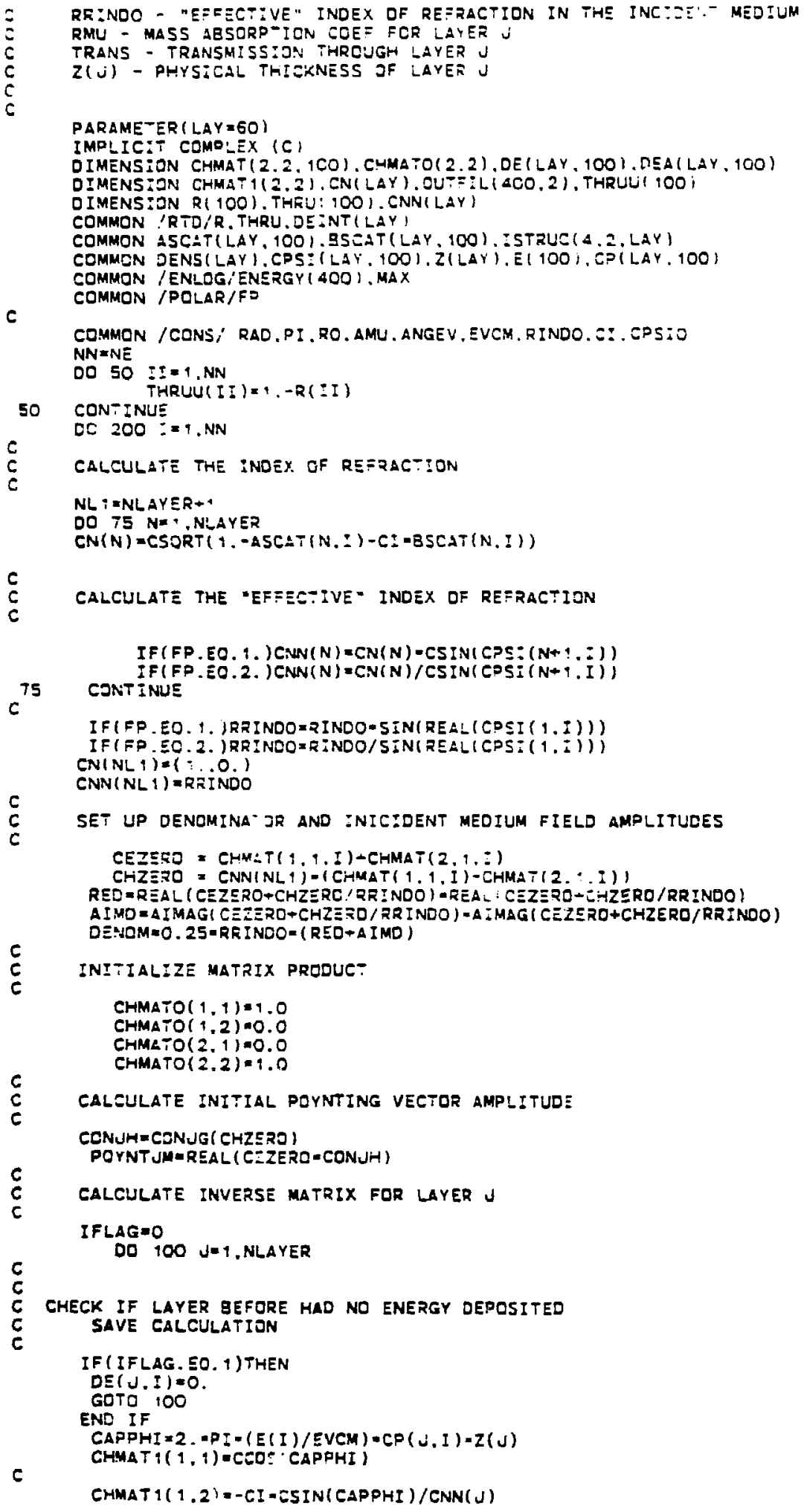


$c$

c

c

C

C

C

c

C

$c$
$C$
$C$
$C$

$$
\text { C }
$$$$
\text { c }
$$$$
\text { C }
$$$$
\text { C = - }
$$$$
\text { C=. }
$$$$
\text { C=- }
$$$$
\mathrm{C}=\mathrm{E}
$$$$
c=-c
$$$$
c=-c
$$$$
c=-c
$$$$
\text { c=. }
$$$$
\mathrm{C}=\mathrm{-}
$$$$
\text { c=n }
$$$$
\text { C=. }
$$$$
100
$$$$
200
$$$$
c
$$

c

300

1950

CHMA: : (2. :)=-EI-CSINICAPOR: )-CNNU)

CHMA-, (2.2)=CCOS1 GAP24:

MULTIP:Y PRODUCT BY NEIW INVERSE

CAL: MMULTI(CHMATO, CHMAT:)

CALCULATE CORRESPONDING ELECTR: AND MAGNETIO FIELD AMDIITUDES AND POYNIING VECTOR AMP!: TUDE

CESUB $=$ CHMATOI, .9$)=C E Z E R O-C H M A T O(1,2)-C H Z E R E$

CHSLBU=CHMATO $(2,1)=C E Z \equiv R C$ - CHMATO $(2,2)=C H Z E R C$

CONJAJE CONUG ( CitSUBU)

CPOYNTJ $=$ CESUEJ $=$ CONUHU

PQYNTJ $=R E A L$ ( SPOYNTU)

TAKE THE OIFFERENCE IN POYNTING VEETOA AMP: ITUDES TO GIVE YOU THE ABSERPTANCE

DE (J.I ) = (DOYNTUM-DOYNTU)/DENOM

IF (DE ( J.I) . LE . O. OR . POYNTU.LT. O. ITREN

IFLAG $=1$

$D E(i,:)=0$

END:F

REINITIALEZE J- 9 POYNTING VECTOR AMPI:TUDE

POYNTJMEDOYNTJ

CALCULATE MORMAL ABSORPTION

GE'T CERRESPONEING MASS IESERPTION COEEFICIENT

IEL-ISTRUC $(1,1, J)$

CAL: FTF2T(IDL. OUTFIL.EMF)

RMUELCT =EMF (EII)

RMU =EINTRP(E(I), QUTFIL(1.2))-RMUFACT

CALCULATE FRACTION TRANSMITTED AND FRACTION ABSCREED

TRANS $=\operatorname{EXP}(-Z(J)=O E N S(J)-R M U, \operatorname{SIN}(\operatorname{REAL}(\operatorname{CPSI}(1.1))))$

AESERP 1 . -TRANS

OEA (U. I) =ABSORP-THRLU(? I

THRUUI I ) = TRANS - THRUU (I ;

CONTINUE

CONTINUE

Do $300 \quad: I=1,1$

WR:TE( 12,1929$)$

FORMAT(" AESORGER JE')

WRITE(12.1950)(DEA(U.II).JE!,NLAYER)

CONTINUE

FORMAT ( 1 PEE 12.4)

RETURN

ENE

SU3ROUTINE MMULTI (CHMAT. CHMAT 9 )

PARAMETER (LAY $=60$ )

COMPLEX CHMAT (2.2). CHMAT $1(2.2), \operatorname{CSLM}(2.2)$

C COMPIEX MATRIX MULTIPLICATION OF 2 MATRICES

$00100 \quad I=1.2$

DO $100 \quad J=1.2$

$\operatorname{CSUM}(I . J)=(0 . .0$.

DO $50 k=9.2$

$50 \operatorname{CSUM}(i, j)=\operatorname{CSUM}(I, J)+\operatorname{CHMAT}:(I, K)=\operatorname{CHMAT}(K, U)$

100 CDNTINUE

DO $200 \quad I=1.2$

Do $200 \quad j=1.2$

$200 \operatorname{CHMAT}(I, U)=$ CSUM $(I, U)$

RETURN

END

C

SURROUTINE SPECTRM(TIME,NPOINTS.E) 


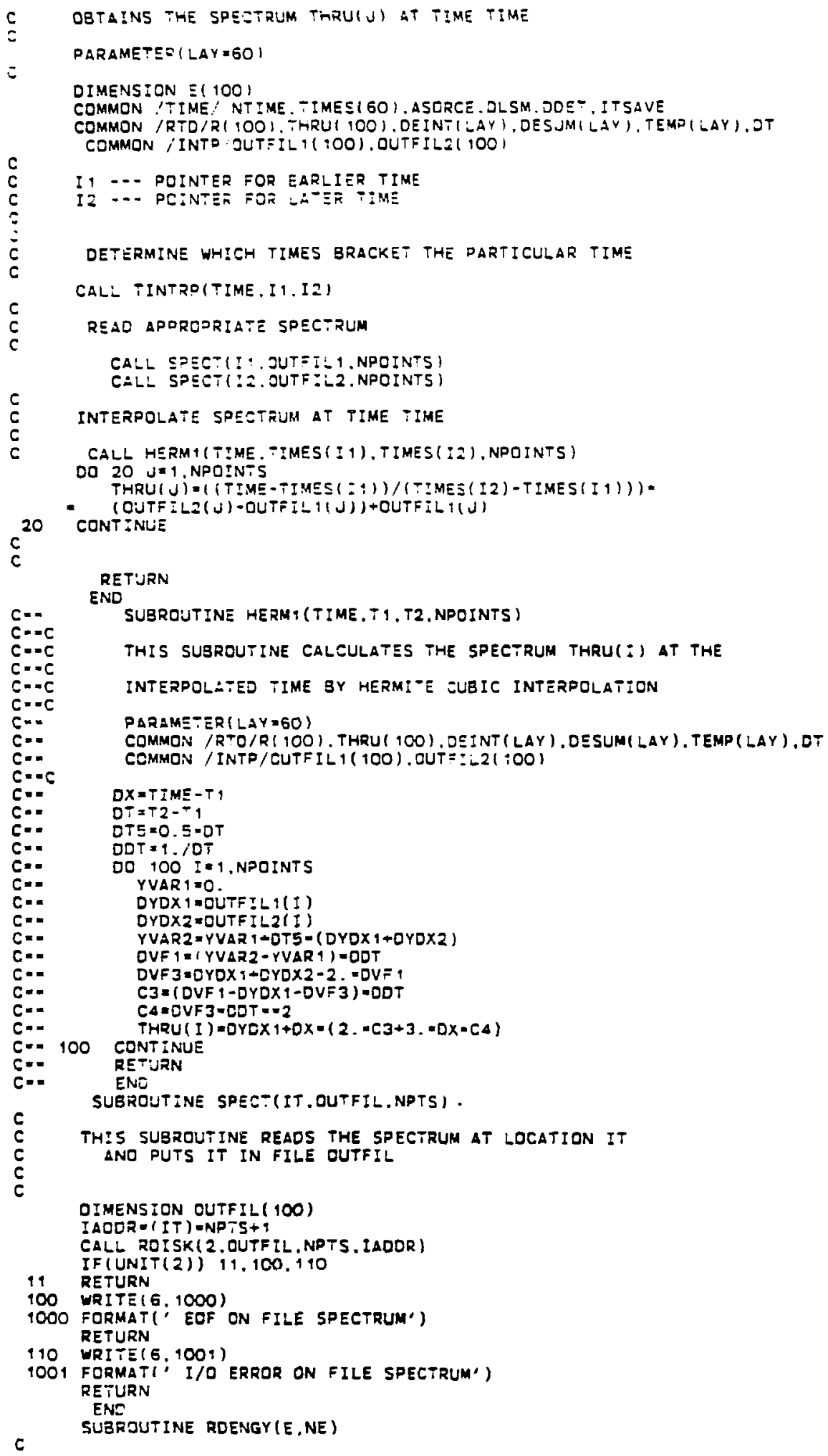


C THIS SUBRJUTiNE READS IN THE NUMBER OF AND ENERGY VALUES

C FOR THE SPEETRUM ARRAYS

OIMENSION E(1)

$I A D D R=C$

CALL ROISK(2.NE, I, IADOR

IF (UNIT (2)) $11,100,110$

$11 \quad I A O D R=I \angle O O R+$

CALL RDISKI =.E, NE, IADDR)

IF (UNIT (2)) 12.100 .110

12 RETURN

100 WRITE(6,1000)

:000 FORMAT(; EDF ON FILE SPECTRUM:)

RETURN

110 WR:TE $16.100: 1$

1004 EDRMATI: I/O ERRDR ON FILE SPECTRUM'। RETUR:J

END

C THIS SUBROUTINE READS THE TIMES THAT SPEZTRUM ARE AVAILABLE

SUBROUTINE RTIME

COMMON/TIME/NT:ME. TIMES(6O) . ASORCE. DLSM, ODET. ITSAVE

REAO IST, 1)NTEME

1 FORMET(I2)

REAO(97.2)(TIMES(J), J-1,NTIME)

2 FORMAT(FA.1)

RETURN

ENE

$\mathbf{C}$
$\mathbf{C}$
$\mathrm{C}$

SUEROUTINE TINTRP(TIME.I1.:Z)

C THIS SUBROUTINE DETERMINES THE TWO TIMES THAT BRACKET

THE TIME FOR INTERPOLAT:ON LATER

CCMMON /TIME/NTIME. TIMESI GOI. ASORCE.DLSM.ODET, ITSAVE

c

DO 100 I=ITSAVE, NTIME

100 CONTINUE

I $2=$ NTT YME

I I =NTIME - I

ITSAVE $=$ I 2

GOT: 170

150 IF $(\mathcal{E}$. EO. 1$)$ THEN

WR IT $=(1,10)$

10 FORMATI' TIME NOT WITHIN OATA BOUNDS ABORTING PROGRAM')

ELSE STOP

I $1=I-1$

END!:

ITSAVE $=I 2$

17C RETURN

END

SUEROUTINE TRANSM(R1,E,N,R2)

C
c
C

THIS SUBROUTINE DETERMINES THE FILTER TRANSMISSION AT ENERGY E AND WULTIPLIES THE TRANSMISSION BY THE SPECTRUM

DIMENSION OUTFIL $(\triangle O C .2\}, R 1(\mathrm{H}) . R 2(N), E(N)$

COMMON /ENLOG;'ENERGY ( 400$)$. MAX

THICK $=123.5 \bar{E}-6$

C DETERMINE M

$I E L=13$

CALL FIF2(IEL, OUTFIL, ATWT, RMU, EMF , NAME)

DO $10 I=1, N$

RMUFACT = EMF/E (I)

RMU =EINTRP (E( $I)$, OUTFIL $(1,2))$ ×RMUFACT

C CALCULATE THE TRANSMISSION

TRANS EXP (-RMU -THICK)

RI(I) FRT(I) -TRAN5

10

$R 2(I)=R 2(I)=$ TRANS

CDNTINUE

RETURN

END 
SUBRCUT:NE SWITCH(CUT.RIN)

THIS SUBROUTINE SWITCHES ARRAY VALUES OF RIN INTE ARRAY OUT

DIMENSION RIN 400.21 , DUT $(\triangle 0 C .2)$

DC $100 \quad I=1,400$

Od $50 \quad J=1.2$

DUTII.J)ERIN(:DU)

SO CONTINUE

100 CONTINUE

RETURN

END

SUEROUTINE FIF2T(IEL. OUTFIL. EMF)

C

THIS SUBRDUTINE GETS THE APPROPRIATE F† AND F2 ANU EMF

COMMON /ENLOG' ENERGY (4OO). MAX

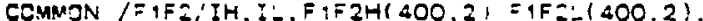

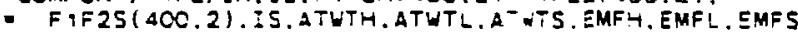

COMMON IEN/MAXH. MAXL. MAXS, EL (4OO ES ( 400$)$, EHI AOO)

C

DIMENSICN DUTE:LL(400.2)

IF(IEL. EO. IH)THEN

CALL SWITCHI DUTFIL, F IF 2Lب)

CALL SWITCHE (ENERGY, MAX. EH, MAXH)

END:F EMF $=$ EMF L

IFI IEL. EO. ILITHEN

CALL SWITCHIOUTFIL FIF 2LI

CALL SWITCHE I ENERGY. MAX. EL. MAXL) EMF A EMFI

ENDIF

IF (IEL.EO. IS)THEN

CALL SWITCH(OUTFIL. F IF2S)

CAL- SWITEHE ( ENERGY, MAX.ES, MAXS)

ENOIF

RETURN

ENC 


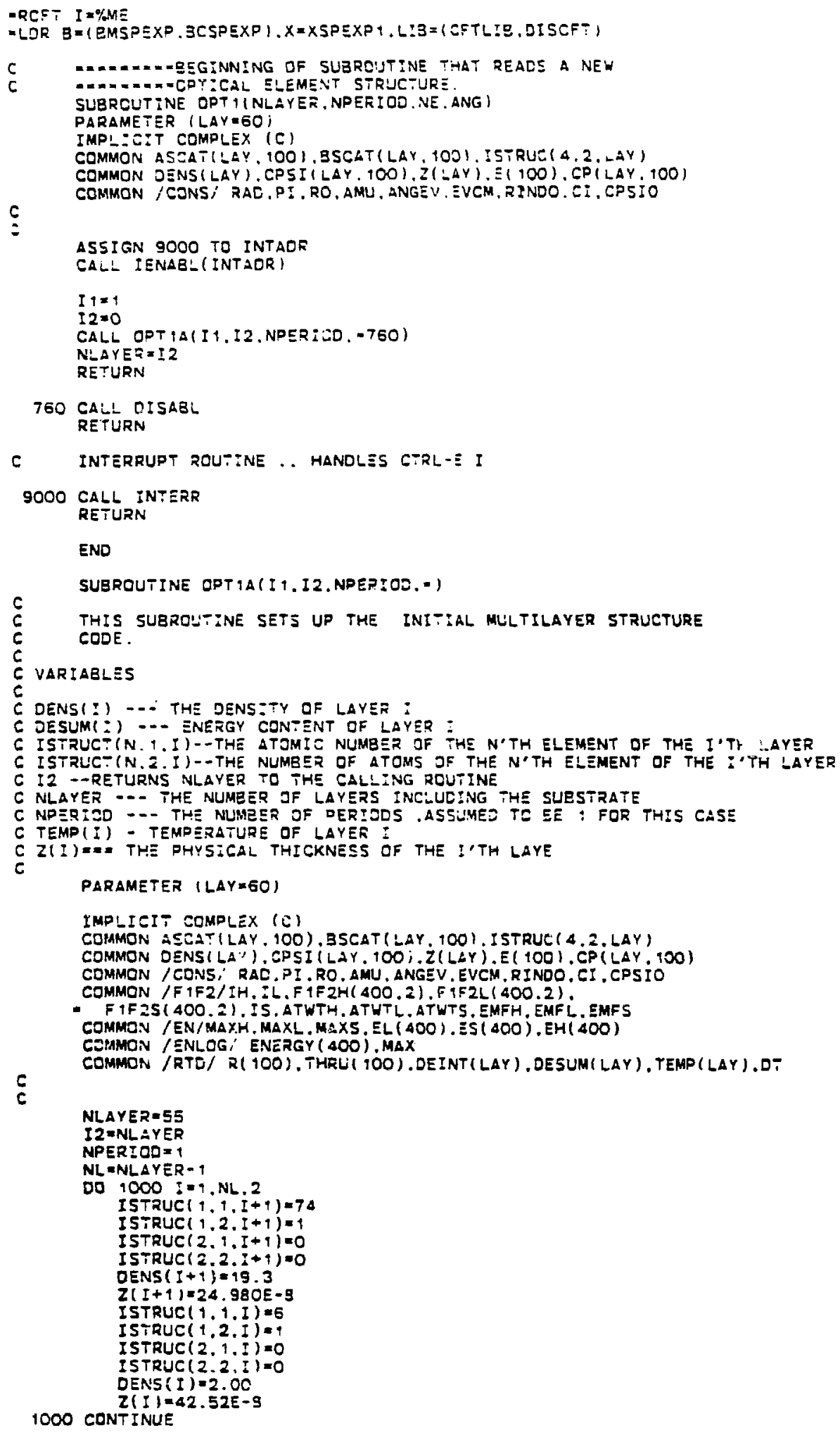




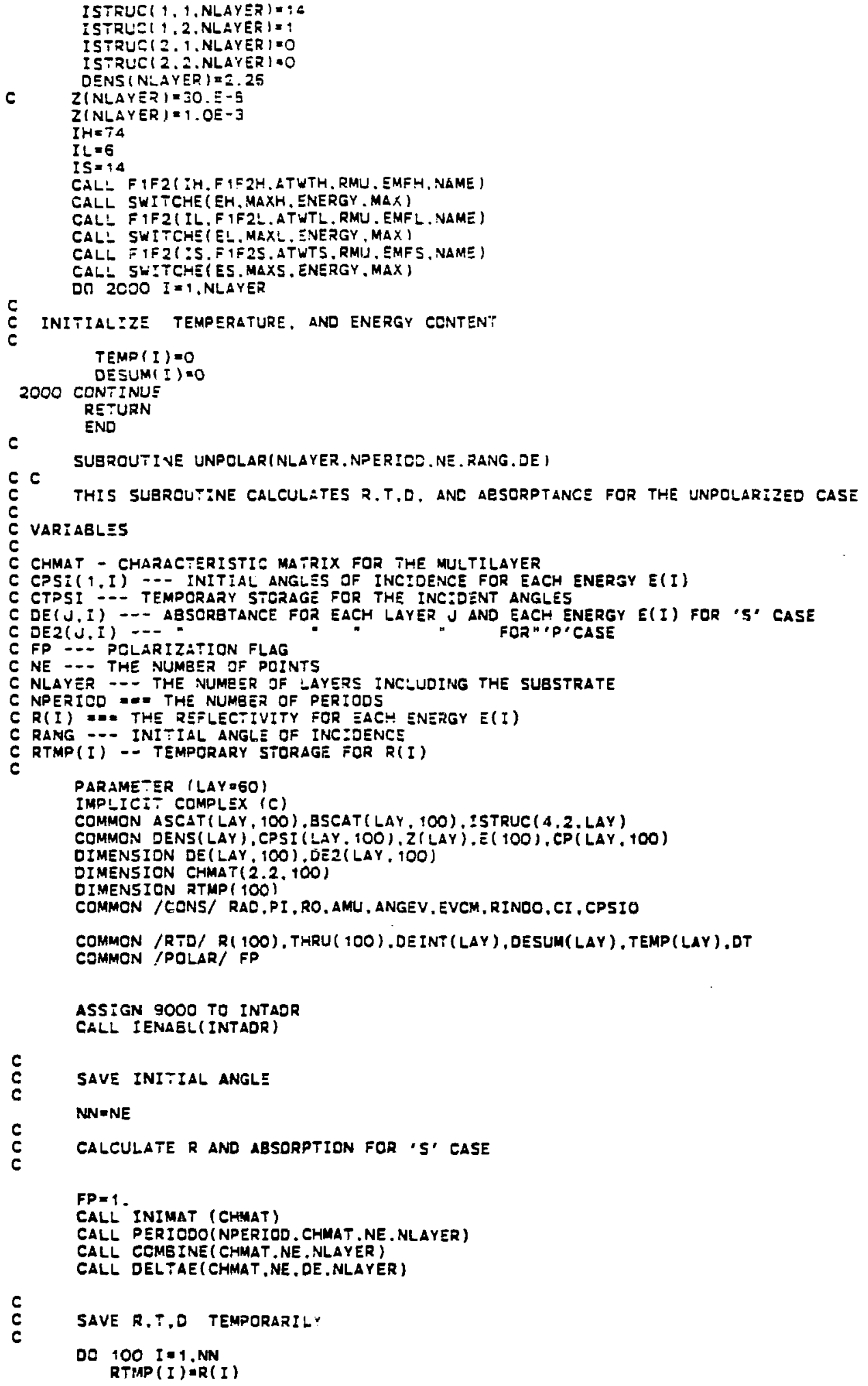




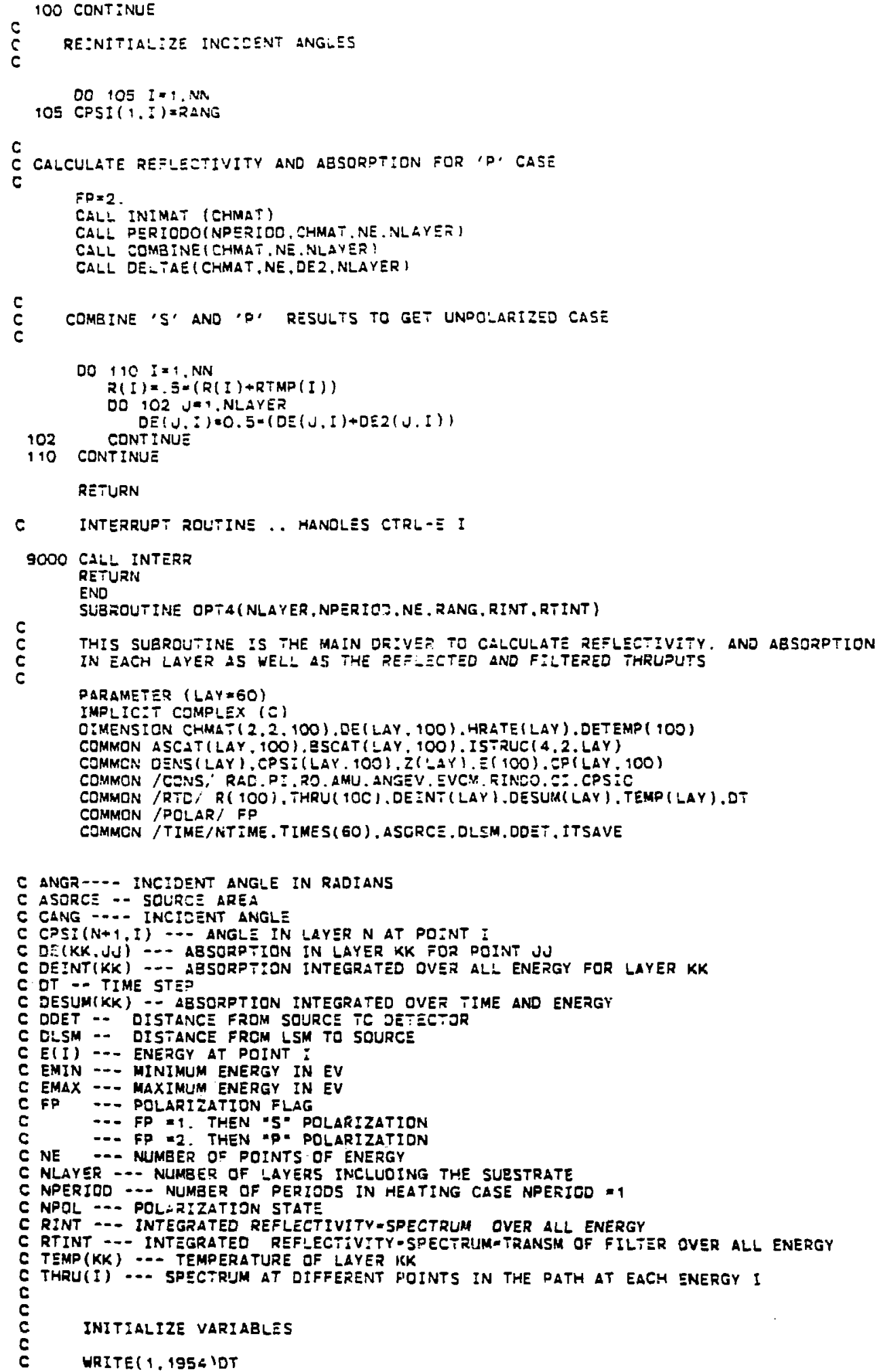




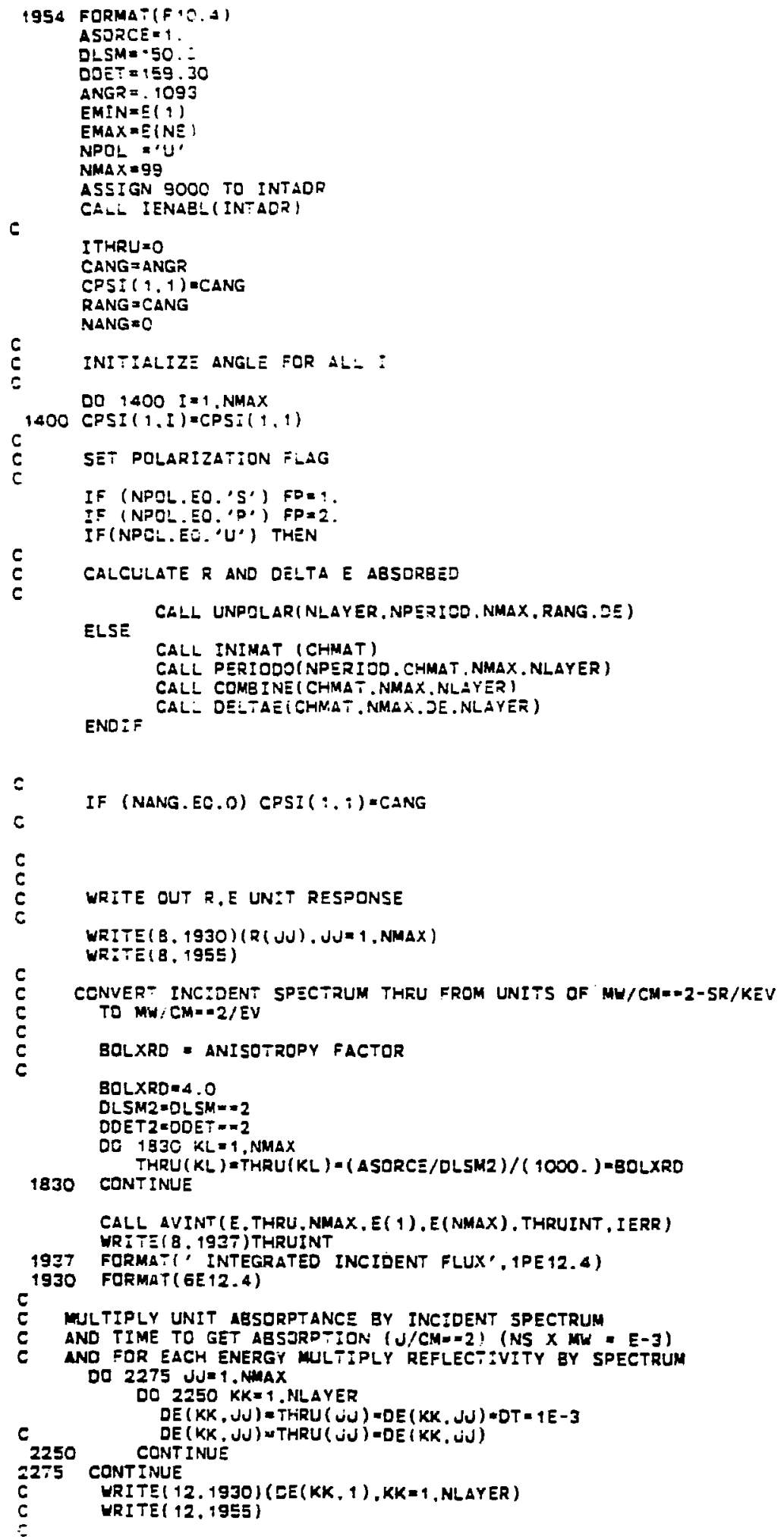




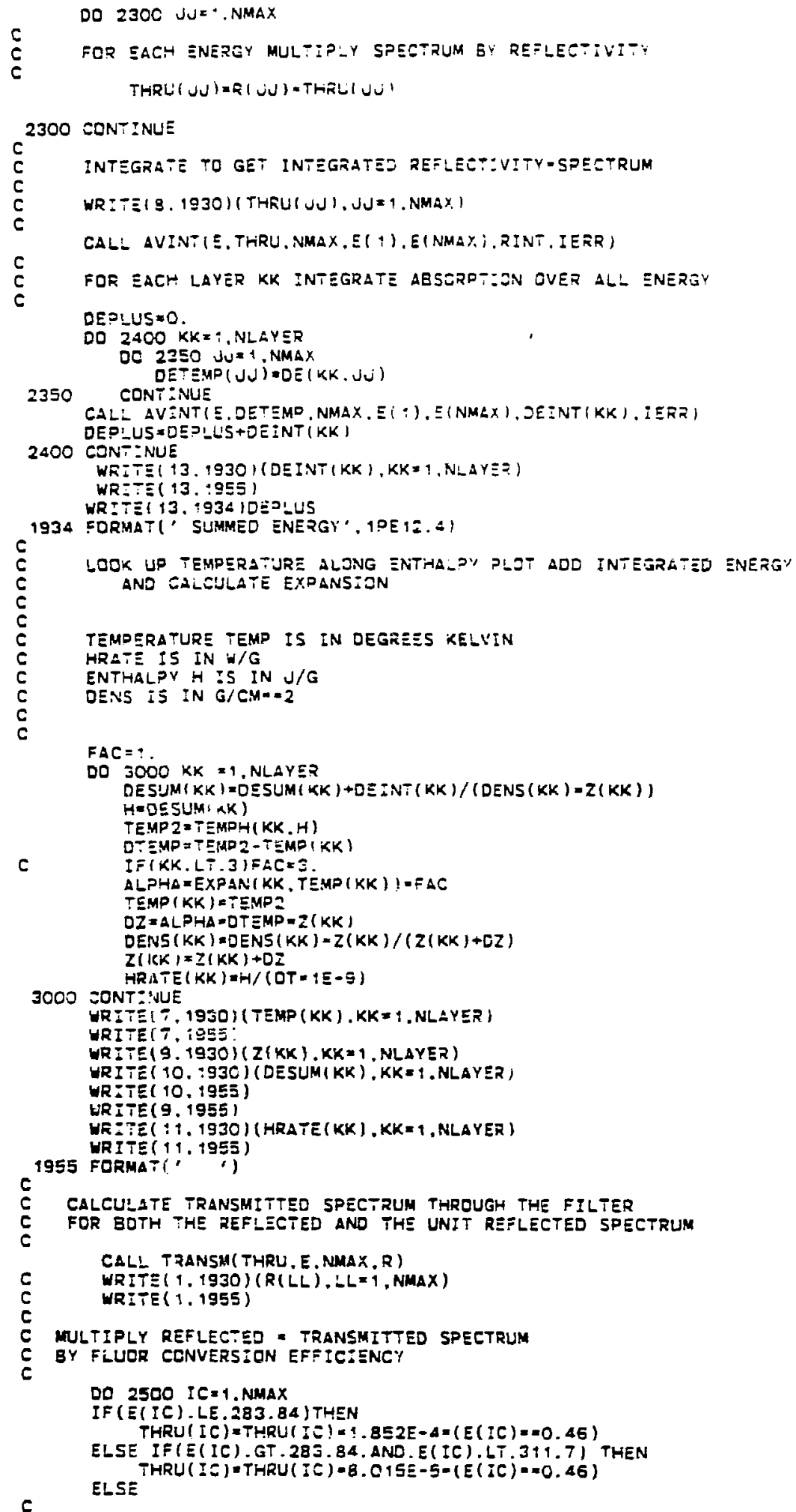




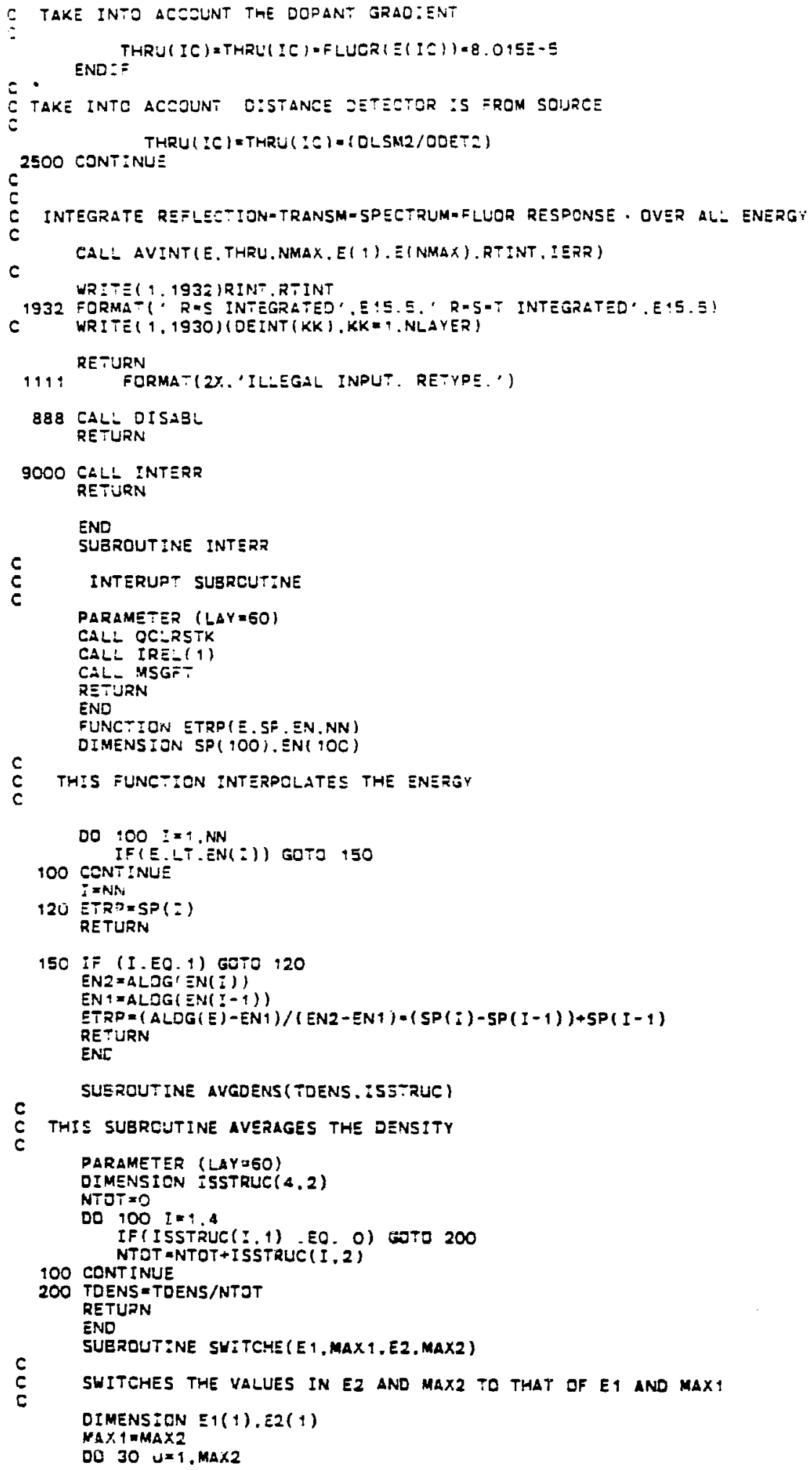




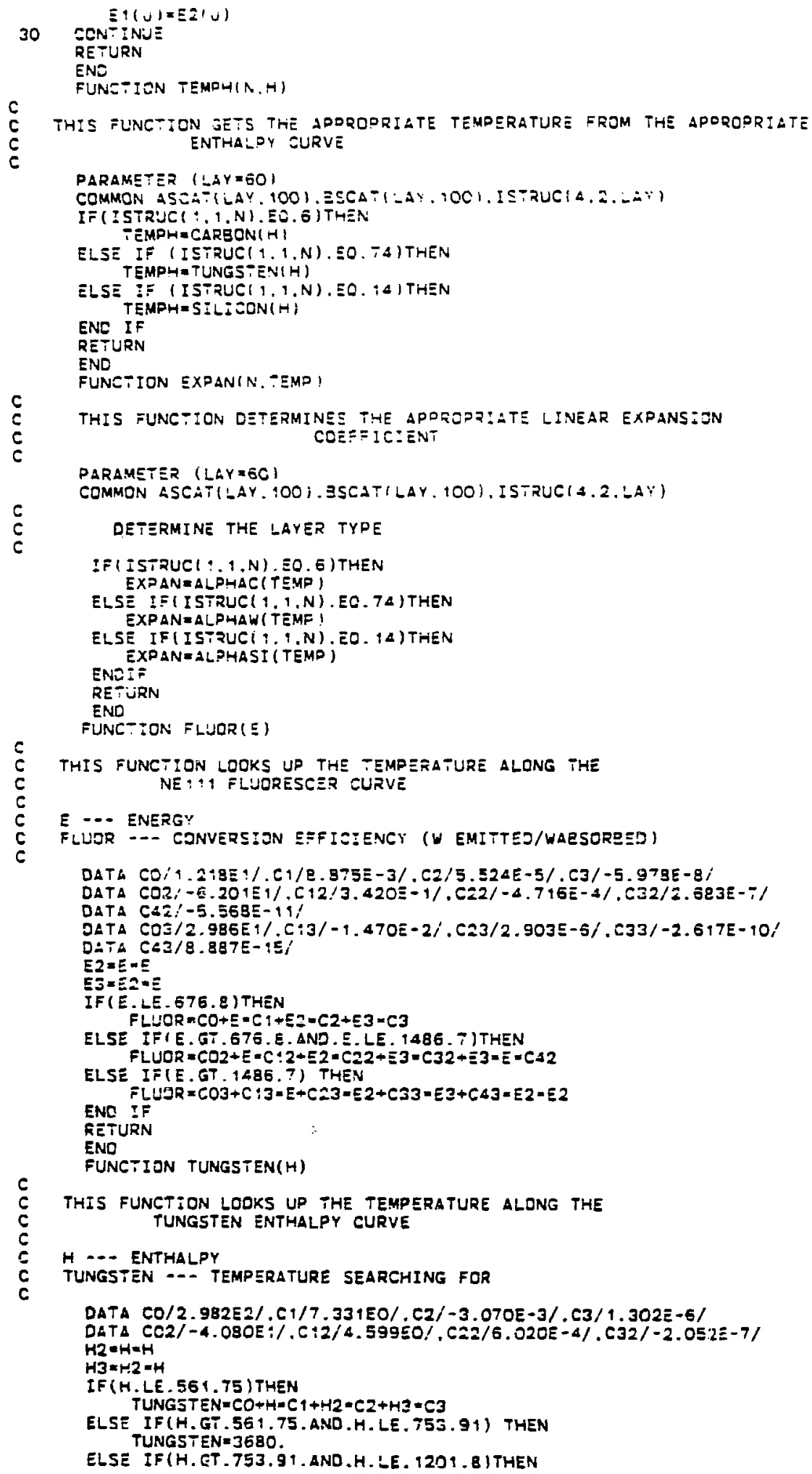




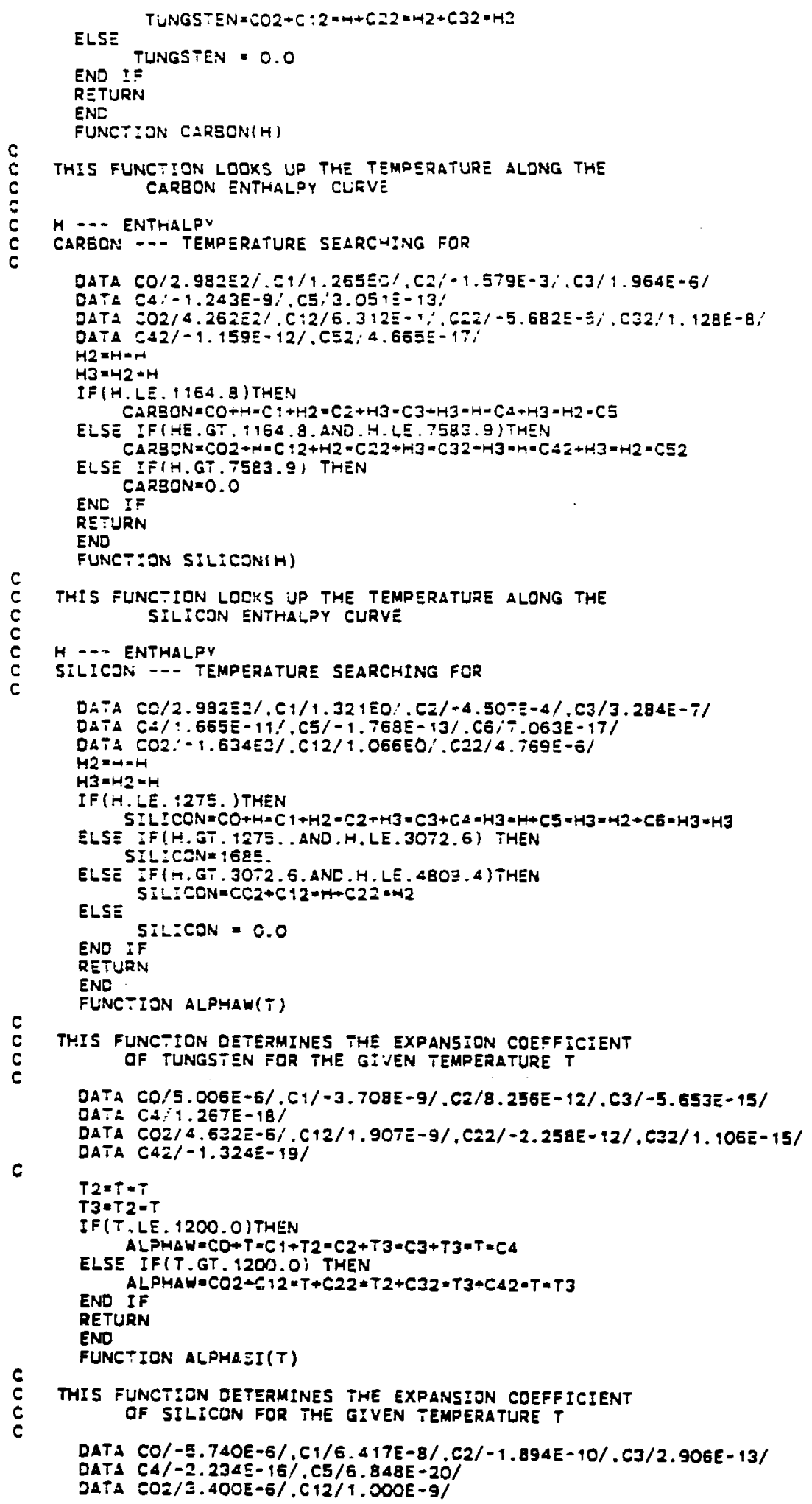




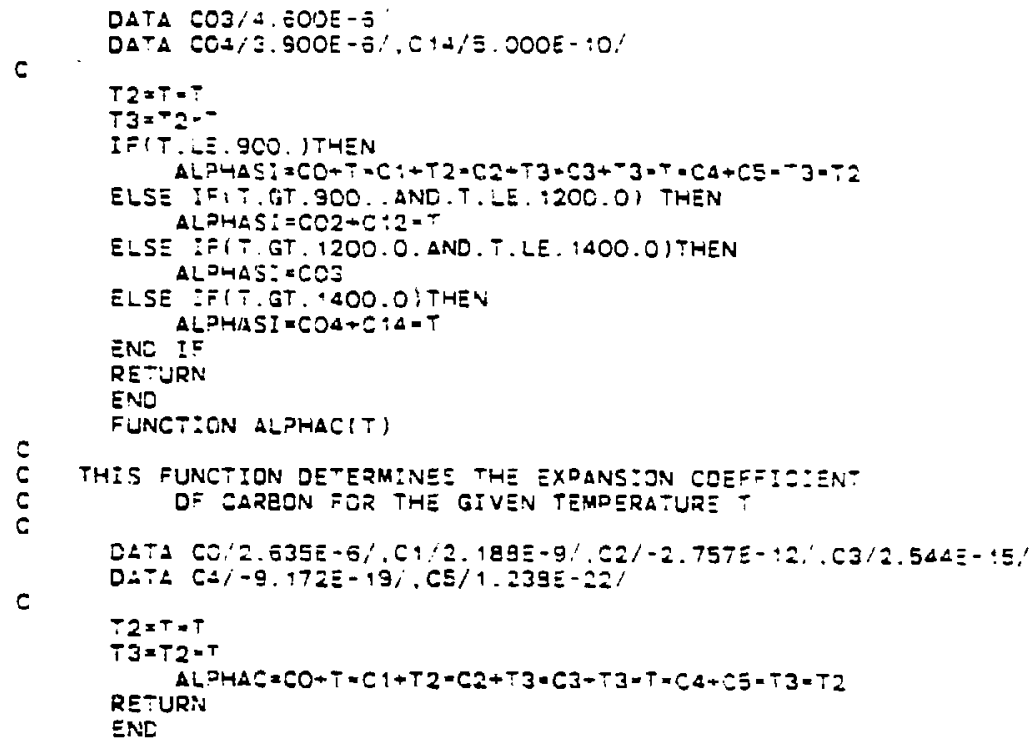

